\title{
WestVirginiaUniversity
}

THE RESEARCH REPOSITORY @ WVU

Graduate Theses, Dissertations, and Problem Reports

2006

\section{Characterization of self -healing composite materials}

\author{
Kevin John Ford \\ West Virginia University
}

Follow this and additional works at: https://researchrepository.wvu.edu/etd

\section{Recommended Citation}

Ford, Kevin John, "Characterization of self -healing composite materials" (2006). Graduate Theses, Dissertations, and Problem Reports. 2732.

https://researchrepository.wvu.edu/etd/2732

This Dissertation is protected by copyright and/or related rights. It has been brought to you by the The Research Repository @ WVU with permission from the rights-holder(s). You are free to use this Dissertation in any way that is permitted by the copyright and related rights legislation that applies to your use. For other uses you must obtain permission from the rights-holder(s) directly, unless additional rights are indicated by a Creative Commons license in the record and/ or on the work itself. This Dissertation has been accepted for inclusion in WVU Graduate Theses, Dissertations, and Problem Reports collection by an authorized administrator of The Research Repository @ WVU.

For more information, please contact researchrepository@mail.wvu.edu. 


\title{
Characterization of Self-Healing Composite Materials
}

\author{
Kevin John Ford \\ Dissertation submitted to the \\ College of Engineering and Mineral Resources \\ at West Virginia University \\ in partial fulfillment of the requirements \\ for the degree of

\section{Doctor of Philosophy} \\ in \\ Aerospace Engineering
}

\author{
Ever J. Barbero, Ph.D., Chair \\ Thomas Damiani, Ph.D. \\ Bruce Kang, Ph.D. \\ Joan Andreu Mayugo, Ph.D. \\ Victor Mucino, Ph.D. \\ Jacky Prucz, Ph.D. \\ Alfred Stiller, Ph.D. \\ Department of Mechanical and Aerospace Engineering \\ Morgantown, West Virginia \\ 2006
}

Keywords: composite materials, damage mechanics, self-healing, encapsulation 


\section{ABSTRACT \\ Characterization of Self-Healing Composite Materials Kevin John Ford}

Damage occurs in almost every composite material in the form of microcracks that develop in the epoxy matrix that binds the fibers together. Researchers at the University of Illinois Urbana Champaign have recently developed a method to reverse the effects of, or heal, damage in the epoxy matrix. Their in-situ self-healing system uses embedded microcapsules and a catalyst that trigger a romp reaction in an effort to rebond the microcracks. Several models have been developed in an effort to predict how a composite laminate damages. One model in particular, the Continuous Damage Mechanics model, CDM that has been developed at West Virginia University uses material properties that are easily obtained from standard ASTM and ISO testing methods. The CDM model has been extended at West Virginia University to incorporate the effects of a self-healing system to develop a Continuous Damage and Healing Mechanics model, CDHM. In this work, a testing procedure to characterize the autonomic healing of polymer matrix composites is outlined, as well as the regenerative effects of the self-healing system. The capability of the CDHM model to predict the material properties of the self-healing system is also addressed. The CDHM model is validated with experimental results for various laminates fabricated out of E-glass/epoxy. 


\section{DEDICATION}

This disertation is dedicated to my family. Your support and encouragement have meant a lot to me over the years and I am forever grateful. Thank you! 


\section{ACKNOWLEDGEMENTS}

I would like to thank my committee members Dr. Thomas Damini, Dr. Bruce Kang, Dr. Joan Andreu Mayugo, Dr. Victor Mucino, Dr. Jacky Prucz, and Dr. Alfred Stiller. I would especially like to thank my advisor Dr. Ever Barbero whom I have worked with the past several years. It has been a pleasure working with you and learning from you. Chuck Coleman and Cliff Judy were a big help on this project. Chuck's help with the lab equipment and Cliff's help in the machine shop were invaluable. Dr. Stansberry thanks for use of the lab space in the ERB to make the microcapsules. Mark Kozlowski and Raymond Claus thanks for your help fabricating and testing the specimens.

I would like to thank my friends for helping me out every now and then on my research project and for there for me. Lloyd thanks for being such a good roommate and friend over the years. Alyson you have been great to me, thank you for your encouragement and for being there to chear me up whenever I would get discouraged.

At last I cannot forget to thank my family. I didn't make it home as many weekends as I'd like to have and I'm thankful for your understanding. Without your support I wouldn't be where I am today. Thanks! 


\section{TABLE OF CONTENTS}

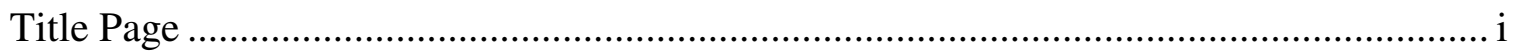

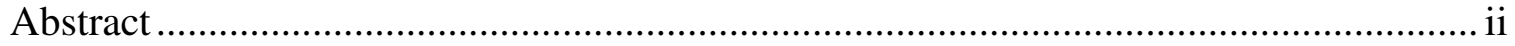

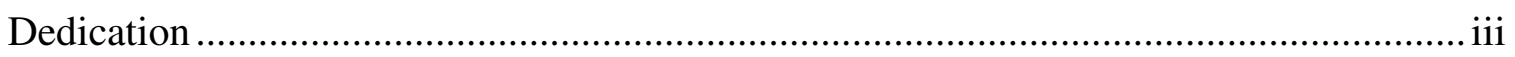

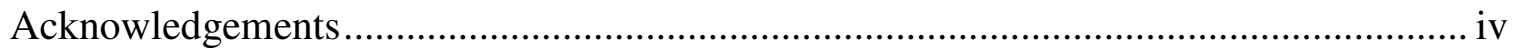

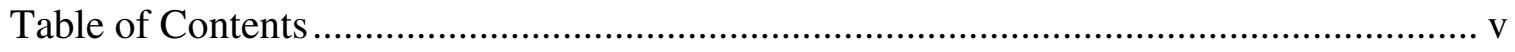

List of Tables ................................................................................................ viii

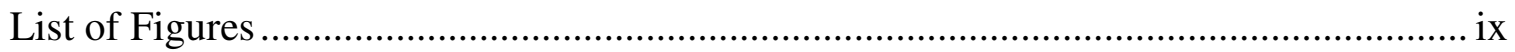

1 Introduction and Literature Review ................................................................. 1

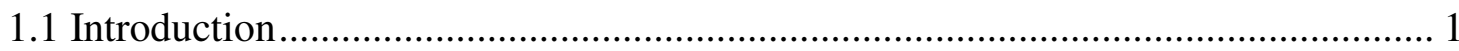

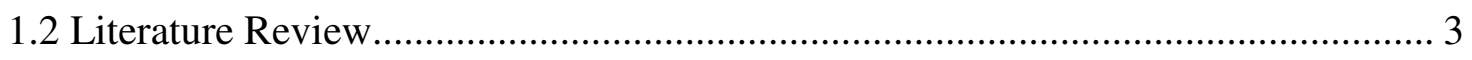

1.2.1 Self-healing Concept in Composite Materials ............................................ 3

1.2.2 Damage Modeling of Composite Materials ............................................... 14

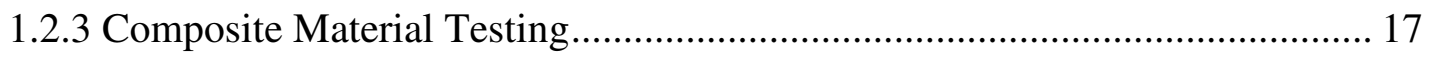

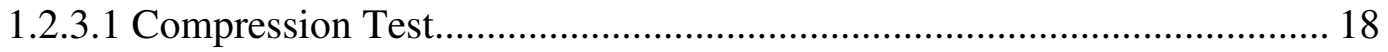

1.2.3.2 Shear Test................................................................................... 18

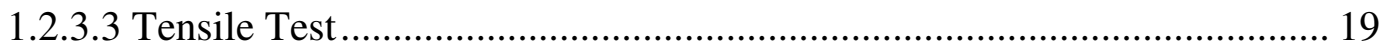

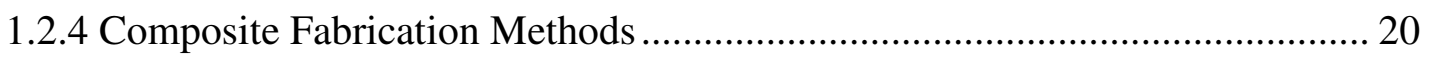

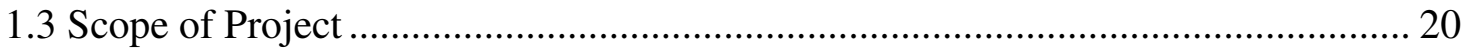

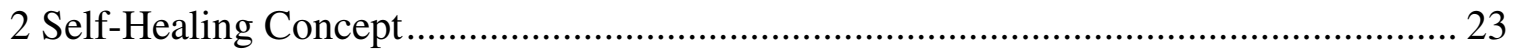

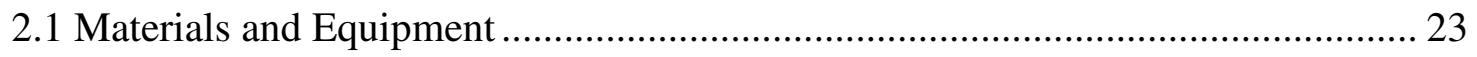

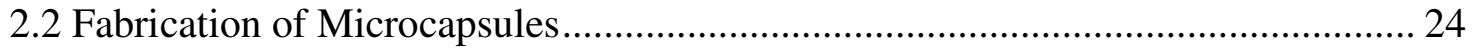

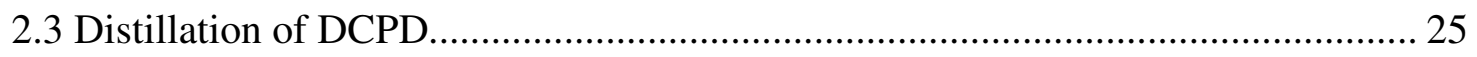




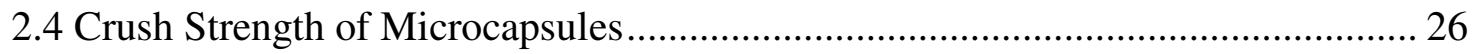

2.5 Wax Encapsulation of Grubbs' Ru Catalyst ....................................................... 28

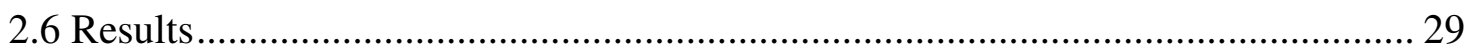

3 Speicmen Fabrication and Testing ............................................................................ 45

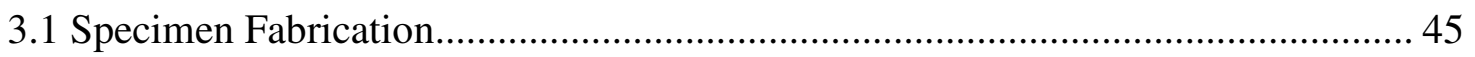

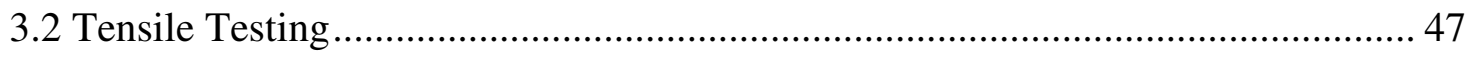

3.3 Compression Testing .................................................................................. 49

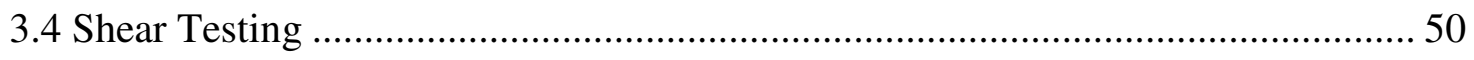

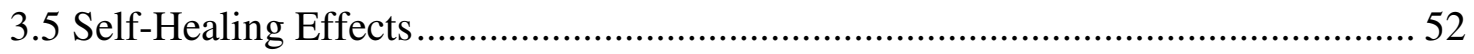

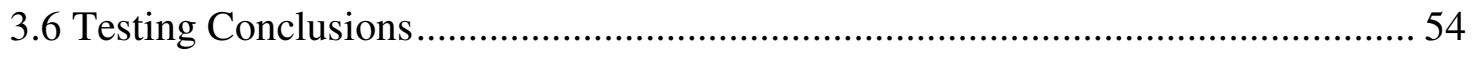

4 Simplified Damage and Healing Mechanics Model ..................................................... 76

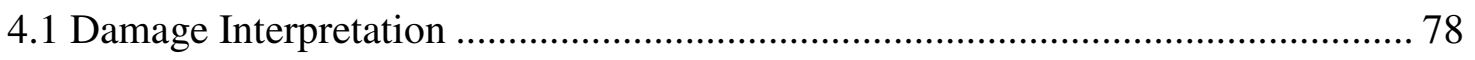

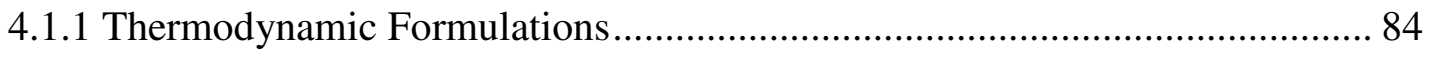

4.1.2 Unrecoverable Deformation, and Damage ……………….......................... 85

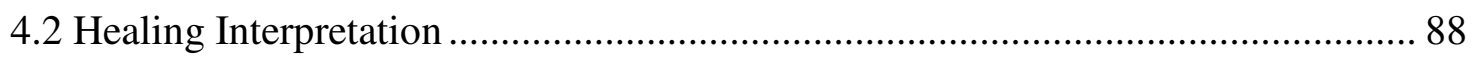

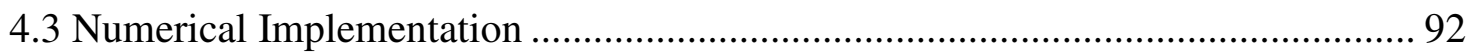

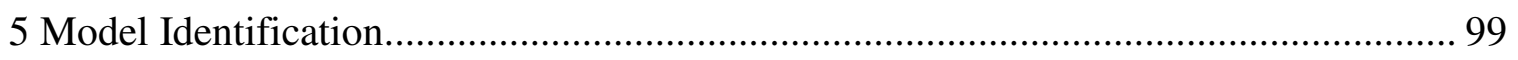

5.1 Determination of the Critical Damage Parameters ................................................ 99

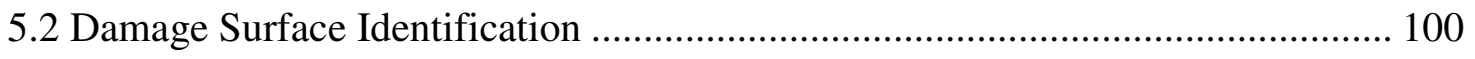

5.3 Unrecoverable-strain Surface Identification ....................................................... 104

5.4 Identification of the Hardening Laws ................................................................ 105

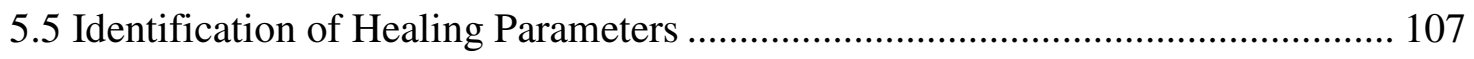

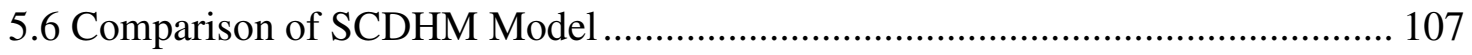


6 Model Validation ......................................................................................... 113

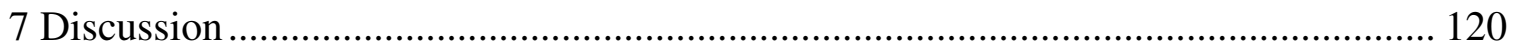

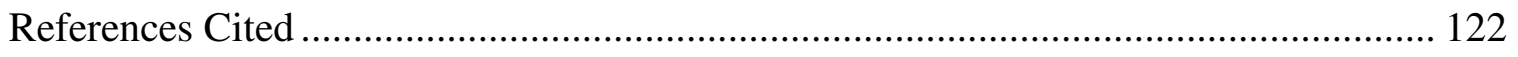

Appendix A Microencapsulation of DCPD Instructions ......................................... 130

Appendix B Comparison of Encapsulation Methods............................................... 131

Appendix C Wax-encapsulation of Grubbs' RU Catalyst Instructions ........................ 133

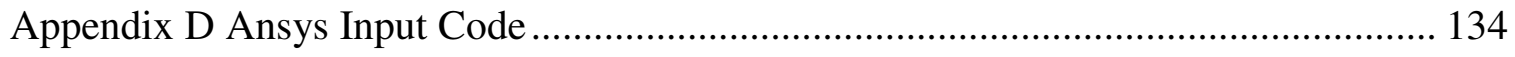




\section{LIST OF TABLES}

Table 2.1 Compression data from microcapsule testing .......................................... 31

Table 3.1 Material properties of specimens containing $20 \%$ wt. of microcapsules and $10 \%$ wt. of wax encapsulated catalyst. 55

Table 3.2 Material properties of fiberglass epoxy not containing the self-healing system

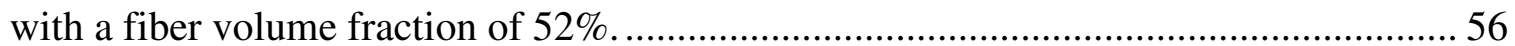

Table 3.3 Comparison of material properties of specimens containing the self-healing system and specimens not containing the self-healing system. 57

Table 5.1 Overall model parameters for damage and plasticity for laminates containing the self-healing system. 109

Table 5.2 Overall adjustable parameters for damage and plasticity for laminates containing the self-healing system. 109

Table 5.3 Healing efficiency parameters 109

Table 5.4 Shear properties for material in Figure 5.3 and Figure 5.4 ......................... 110 Table 5.5 Model parameters for damage and plasticity for the specimen in Figure 5.3 and Figure 5.4. 110

Table 5.6 Overall adjustable parameters for damage and plasticity for the specimen in

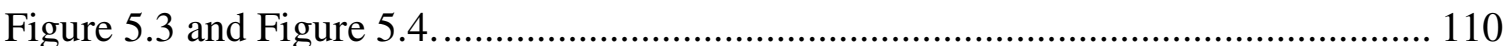




\section{LIST OF FIGURES}

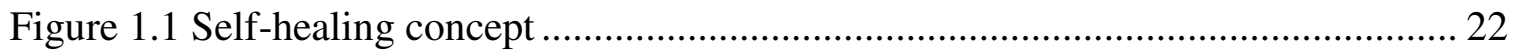

Figure 2.1 Flowchart outlining the fabrication process of microcapsules....................... 32

Figure 2.2 Experimental setup used in the fabrication of the microcapsules. .................. 33

Figure 2.3 Close-up of oil-in-water emulsion during the microencapsulation process.... 34

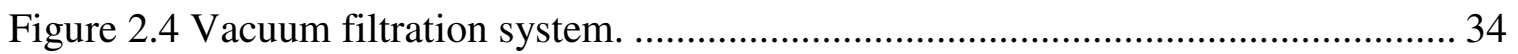

Figure 2.5 Microcapsule cakes drying in vacuum filter. ............................................. 35

Figure 2.6 Gilson Performer III sieve shaker and sieve trays......................................... 35

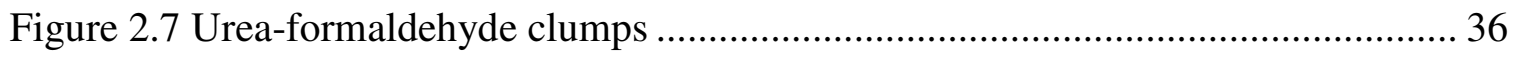

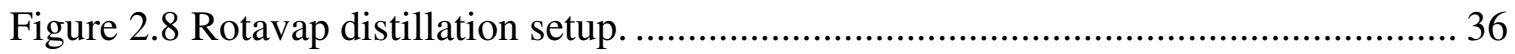

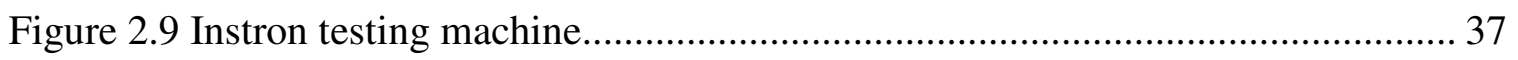

Figure 2.10 Compression test apparatus …………………………………………... 37

Figure 2.11 Stress vs. strain compression data form microcapsules................................ 38

Figure $2.1253 \mu \mathrm{m}$ diameter stress strain curve ..................................................... 38

Figure $2.13106 \mu \mathrm{m}$ diameter stress strain curve ....................................................... 39

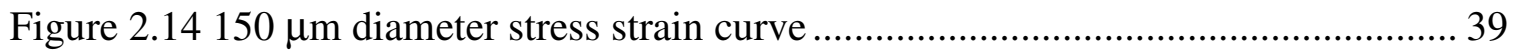

Figure $2.15212 \mu \mathrm{m}$ diameter stress strain curve ........................................................... 40

Figure 2.16 Microcapsules strength versus microcapsule size. ……………………..... 40

Figure 2.17 Size distribution of microcapsules at agitation rates of $550 \mathrm{rpm}, 850 \mathrm{rpm}$, and

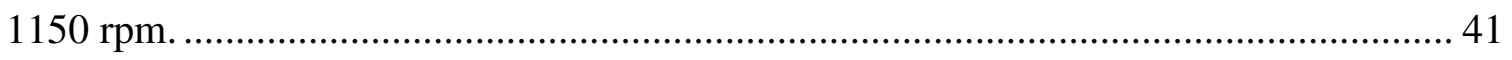

Figure 2.18 Microcapsules and urea-formaldehyde clumps at x60 magnification........... 41

Figure 2.19 Microcapsules, approximately $200 \mu \mathrm{m}$ in diameter, x400 magnification and x300 magnification respectively. 42 
Figure 2.20 Microcapsule outer surface, notice the roughness of the outer surface, the shell wall is smooth, the leftover urea-formaldehyde clumps adhere to the surface making it rough, $\mathrm{x} 20.2 \mathrm{k}$ and $\mathrm{x} 15.0 \mathrm{k}$ magnification, respectively 42

Figure 2.21 Broken microcapsule, notice the inner surface is smooth, compared to the rough outer surface $\mathrm{x} 500$

Figure 2.22 Broken microcapsule shell wall, approximately $0.30 \mu \mathrm{m}$ thick $\mathrm{x} 40.0 \mathrm{k}$ magnification 43

Figure 2.23 Grubbs' Ru catalyst encapsulated in wax at x30 magnification. 44

Figure 3.1 Hand lay-up and vacuum bagging of samples........................................ 58

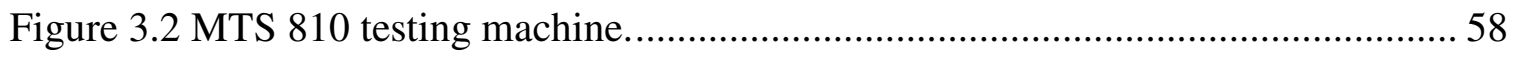

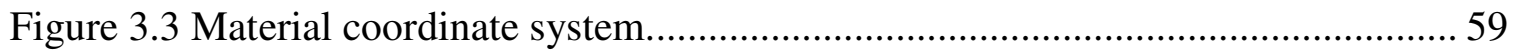

Figure 3.4 Tensile test data in the longitudinal direction for specimens containing the self-healing system. 59

Figure 3.5 Tensile test data in the transverse direction for specimens containing the selfhealing system. 60 Figure 3.6 Tensile test data in the longitudinal direction for specimens not containing the

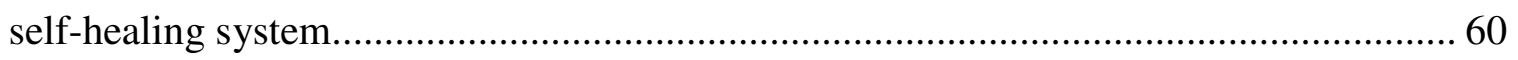
Figure 3.7 Tensile test data in the transverse direction for specimens not containing the

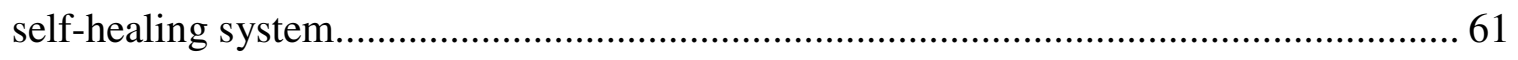
Figure 3.8 SACMA SRM 1R-94 compression test specimen (all dimensions are given in

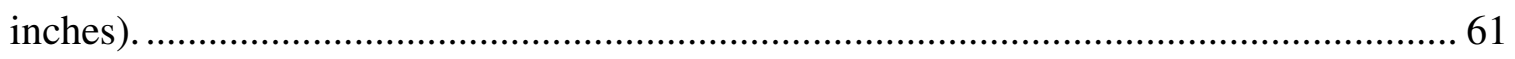

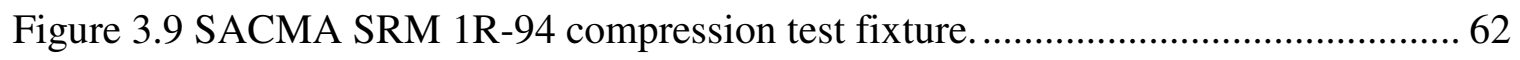

Figure 3.10 Instron testing machine with SACMA compression fixture. ....................... 62 
Figure 3.11 Compression test data for specimens containing the self-healing system in the longitudinal direction.

Figure 3.12 Compression test data for specimens containing the self-healing system in the transverse direction. 63

Figure 3.13 Compression test data for specimens not containing the self-healing system in the longitudinal direction. 64

Figure 3.14 Compression test data for specimens not containing the self-healing system in the transverse direction. 64

Figure 3.15 Iosipescu test specimen (all dimensions are given in $\mathrm{mm}$ ). 65

Figure 3.16 Iosipescu test fixture diagram and picture of test fixture. 66

Figure 3.17 Shear data for specimens containing the self-healing system in the ' $1-2$ direction'. 67

Figure 3.18 Shear data for specimens not containing the self-healing system in the '1-2 direction'. 67

Figure 3.19 Cyclic loading of shear specimen without self-healing system. 68 Figure 3.20 Comparison of the shear loading curves from the specimen in Figure 3.19 moved to the origin. 68

Figure 3.21 Cyclic shear slopes $\left(\mathrm{G}_{12}\right)$ vs. loading number of specimen in Figure 3.19 and Figure 3.20. Notice that with each reloading the slope decreases.

Figure 3.22 Specimen with self-healing system loaded to $2.5 \%$ strain unloaded, not allowed to heal, then reloaded. 69

Figure 3.23 Specimen with self-healing system loaded to $1.5 \%$ strain, allowed to heal (48 hrs), then reloaded. 70 
Figure 3.24 Specimen with self-healing system loaded to $2.5 \%$ strain, allowed to heal (48 hrs), then reloaded. 70

Figure 3.25 Specimen with self-healing system loaded to $3.0 \%$ strain, allowed to heal (48 hrs), then reloaded. 71

Figure 3.26 Typical cyclic healing shear test........................................................ 71

Figure 3.27 Cyclic healing shear testing, all data is shifted to the origin....................... 72

Figure 3.28 Cyclic shear slopes $\left(\mathrm{G}_{12}\right)$ vs. loading number of specimen in Figure 3.27. Notice that with slope each reloading the slope does not necessarily decrease............... 72 Figure 3.29 Cyclic healing shear testing, all data is shifted to the origin. 73

Figure 3.30 Cyclic shear slopes $\left(\mathrm{G}_{12}\right)$ vs. loading number of specimen in Figure 3.29. Notice that with slope each reloading the slope does not necessarily decrease............... 73 Figure 3.31 Cyclic healing shear testing, all data is shifted to the origin. 74

Figure 3.32 Cyclic shear slopes $\left(\mathrm{G}_{12}\right)$ vs. loading number of specimen in Figure 3.31 . Notice that with slope each reloading the slope does not necessarily decrease....... 74 Figure 3.33 Comparison of cyclic shear slopes $\left(\mathrm{G}_{12}\right)$ vs. loading number of specimens not containing the self-healing system, specimens containing the self-healing system that did not heal, and specimens containing the self-healing system that healed...................... 75

Figure 4.1 Diagram of the damage/healing domain and damage threshold. .................. 97 Figure 4.2 Shear loading cycle for a self-healing composite material depicting the initial shear modulus, damaged shear modulus, and healed shear moduls. ........................... 97

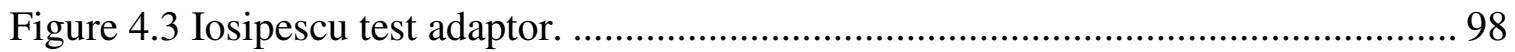
Figure 5.1 Typical shear stress versus shear strain loading and unloading curve. ........ 111 Figure 5.2 Healing efficiency versus transverse damage....................................... 111 
Figure 5.3 Comparison of CDHM model and experimental data for the first loading of a [0/90] laminate under shear loading. 112

Figure 5.4 Comparison of CDHM model and experimental data for the second (healed) loading of a [0/90] laminate under shear loading. 112

Figure 6.1 ANSYS model of a sixteen layer laminate using symmetry conditions. ...... 115

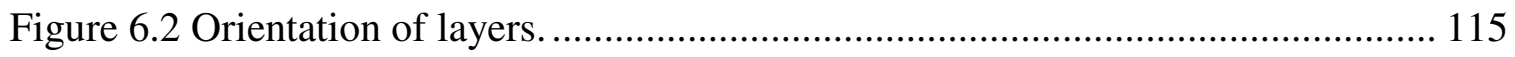

Figure 6.3 Coupling of nodes using periodic boundary conditions........................... 116

Figure 6.4 Comparison of the first loading and the second (healed) loading of a $\left[[0 / 90]_{3} /+45 /-45\right]_{S}$ laminate that was loaded to $1.5 \%$ shear strain.

Figure 6.5 Comparison of the first loading of model and experimental data of a $\left[[0 / 90]_{3} /+45 /-45\right]_{s}$ laminate tested in cyclic healing loading at $1.5 \%$ strain increments.

Figure 6.6 Comparison of the second (healed) loading of model and experimental data of $\mathrm{a}\left[[0 / 90]_{3} /+45 /-45\right]_{S}$ laminate tested in cyclic healing loading at $1.5 \%$ strain increments. 117

Figure 6.7 Comparison of the first loading and the second (healed) loading of a $\left[[0 / 90]_{3} /+45 /-45\right]_{s}$ laminate that was loaded to $2.25 \%$ shear strain. 118

Figure 6.8 Comparison of the first loading of model and experimental data of a $\left[[0 / 90]_{3} /+45 /-45\right]_{S}$ laminate tested in cyclic healing loading at $2.25 \%$ strain increments. 
Figure 6.9 Comparison of the second (healed) loading of model and experimental data of a $\left[[0 / 90]_{3} /+45 /-45\right]_{s}$ laminate tested in cyclic healing loading at $2.25 \%$ strain

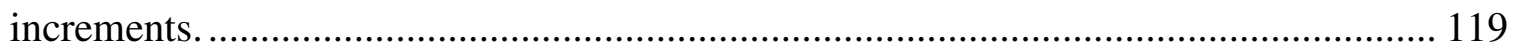




\section{INTRODUCTION AND LITERATURE REVIEW}

\subsection{Introduction}

Composite materials are formed by the combination of two or more distinct materials to form a new material with enhanced properties [13]. Wood, human bone, and concrete are all considered composite materials. The use of composite materials is growing, their use is not limited to just space shuttles or stealth aircraft. Most people of have heard of fiberglass boats or carbon arrow shafts and fishing rods. The most common types of composite materials in industry consist of a fiber and matrix. Fiberglass, carbon fiber, $\operatorname{Kevlar}{ }^{\circledR}$, and boron are typical fibers that are bound together with epoxies, polyesters, vinyl ester resins, and other matrix materials. One of the main driving forces for using composite materials is their high strength to weight ratio. Another advantage is that they can be used to fabricate complex shapes.

Over the past several years, two novel developments in the field of composite materials have been made. One development deals with autonomic healing or selfhealing of composite materials $[11,22,23,24,31,34,39,41,43,44,45,55,56,63,79$, 81, 82]. One system in particular, the self-healing system developed at the University of Illinois Urbana Champaign, incorporates the use of urea-formaldehyde microcapsules filled with dicyclopentadiene (DCPD), a healing agent, which are ruptured by the onset of a microcrack in the composite laminate. These microcapsules along with the catalyst are uniformly dispersed in the matrix material (Figure 1Figure 1.1 I). Once ruptured the microcapsule releases the DCPD through capillary action into the propagated crack where it comes into contact with a catalyst, Grubbs' Ru catalyst [74] (Figure 1 II). The following chemical reaction creates a living polymer $[42,70]$ that fills the void made by 
the microcrack, healing the composite lamina (Figure 1 III). The efficiency of the repair depends on the catalyst and the microcapsule concentrations. Some research as to the effects of the overall material properties of a composite containing the self-healing system has been done. The existing body of research deals with the study of fracture toughness of double cantilever beam and tapered cantilever beam specimens [23, 24, 82]. The existing research looks at the effects of catalyst and microcapsule concentrations in the composite but does not deal with effects on the overall material properties of the lamina.

The other development is the Continuous Damage Mechanics theory and other damage theories from which several numerical models have been developed that describe the damage response of composite materials $[14,16,17,18,30,40,49,50,62,75,87$, 88, and 89]. One particular model developed by Barbero et al. is defined using Continuous Damage Mechanics coupled with Classical Thermodynamic Theory and Classical Plasticity Theory [17 and 18]. The Continuous Damage Theory is a branch of Continuum Mechanics that describes the gradual evolution of planar microdefects and cavitation of spheroidal microvoids [49]. Many Continuous Damage Mechanics models available in the literature use a large number of parameters and no clear procedure is revealed to identify (measure) those parameters in terms of available or feasible tests [16]. When those procedures are revealed, the required tests are not standard; no data is available other than that provided by the proponent. The model developed by Barbero et al., the Continuum Damage Mechanics (CDM) model, is unique in that the required parameters can be obtained from standard ASTM and or ISO testing. 
Barbero et al. further developed their CDM model to include the effects of selfhealing materials. An expanded version of the CDM model, Continuum DamageHealing Mechanics (CDHM) [19]) is a constitutive model based on a continuum thermodynamic framework.

The CDM model was developed to use existing data already available in the literature. Only data dealing with the fracture toughness of self-healing composite materials is currently present. Where the CDM model can and has been easily validated with experimental data, the CDHM model has been neither experimentally identified nor validated. Other healing processes such as geological rock densification [59], selfhealing healing of concrete $[36,37,68]$, and self-healing healing of ceramic materials $[4$,

5] have been discussed in the literature. Numerical modeling, of the above-mentioned processes, has not been completely investigated. Some models for bone remodeling or wounded skin regeneration have been developed for relatively simple cases $[1,76]$. A constitutive model for compaction of crushed rock salt has been proposed in the thermodynamic framework [58]. The current research, discussed in this dissertation, combines all of the questions raised about the effects of the self-healing system and the validation of the CDHM model.

\subsection{Literature Review}

\subsubsection{Self-healing Concept in Composite Materials}

Researchers at the University of Illinois at Urbana-Champaign, UIUC, have recently developed a polymeric material with the ability to autonomically heal cracks [22, $23,24,41,81]$. Through fracture experiments they were able to yield as much as $75 \%$ recovery in toughness. Other crack-healing methods that have been reported have 
successfully healed cracks in several types of materials such as glass, concrete, asphalt and polymers, though some sort of manual intervention was needed [23]. White et al.'s [81] approach to crack healing is self-initiated and no manual intervention is needed. Microencapsulated healing agents and a catalytic chemical trigger are embedded within an epoxy matrix. Once crack propagation occurs and reaches the embedded microcapsules, the cracks rupture the microcapsules releasing the healing agent. This healing agent, through capillary action then comes in contact with the catalyst. Polymerization of the healing agent is then triggered and bonds the crack faces. Their self-healing system uses the ROMP reaction of dicyclopentadiene [42] with Grubbs' Ru catalyst [74]. This ROMP reaction has a major advantage over two part epoxy polymerization reactions. The heterogeneous nature of this reaction does require a precise stoichiometry ratio. That is, it can be triggered by discrete mixing at a low concentration [23].

Several variations of the encapsulation process of dicyclopentadiene have been discussed in the literature $[22,43,81]$. The concept behind the fabrication of the microcapsules is best outlined in Kessler [43]. Deionized water and ethylene maleic anhydride copolymer are added to a beaker. Next, urea, ammonium chloride, and resorcinol are dissolved in the solution under agitation by a mixer. The $\mathrm{pH}$ of the system is then adjusted to 3.5 and 1-octonal is then added to eliminate surface bubbles. Distilled dicyclopentadiene is then poured into the beaker. Formaldehyde is then added to the solution, which is then brought to temperature and kept at temperature, under agitation. A more in-depth outline of this process is discussed in Chapter 2. Further work in the area of in-situ encapsulation techniques have been discussed by Baxter, Thies, and 
Arshayd and George. Dietrich et al. discuss in-situ encapsulation of water-immiscible liquids by the reaction of urea with formaldehyde at acid $\mathrm{pH}$. Tan et al., Yan et al., Alexandridou and Kiparissides, and Ovez et al. have all shown that by adjusting the agitation rate during microencapsulation one can control the size of the microcapsules. The above-mentioned work was used in the development of the microencapsulation process developed at UIUC.

It was found that the agitation rate determined the diameter of the microcapsule $[22,43]$. As the agitation rate was increased the average diameter of the microcapsules decreased. Brown et al. were able to produce microcapsules with a $10-1000 \mu \mathrm{m}$ range by adjusting the agitation rate between 200-2000 rpm. They determined that a linear relationship in log-log scale existed between the average diameter and the agitations rate. Brown et al. [22] were able to achieve $80-90 \%$ yields of microcapsules that are in the form of a free flowing powder. Through the use of Carbon-Hydrogen-Nitrogen elemental analysis they determined that the fill content of the microcapsules is 83-92 wt.\% DCPD and 6-12 wt.\% urea-formaldehyde. The remaining weight was accounted for by water absorption. They noticed that the average fill content of the microcapsules decreased by 2.3 wt. $\%$ after sitting in ambient laboratory conditions for 30 days.

Brown et al. [22] discussed the effects of the shell wall thickness and the outer surface of the shell. The strength and permeability of the microcapsules is controlled by the shell wall thickness. It is important that the shell wall is strong enough to keep the capsule from breaking during the lay-up process, yet still rupture when the crack reaches the microcapsule. Kessler [43] studied urea formaldehyde microcapsules from the Minnesota Mining and Manufacturing Company (3M) and from Thies Technologies, as 
well as UIUC. The $3 \mathrm{M}$ microcapsules have a shell wall thickness of approximately 1.3 $\mu \mathrm{m}$, Thies Technologies' microcapsules have a shell wall thickness of approximately $0.75 \mu \mathrm{m}$, and the UIUC microcapsules have a shell wall of approximately $0.20 \mu \mathrm{m}$. Kessler further studied the surface morphology of the microcapsules. He noticed a unique feature of the UIUC microcapsules. Urea-formaldehyde debris accumulated on the outer surface of the shell wall, creating a complex globular morphology on the microcapsule wall. The accumulation on the outer surface of the shell wall increases the surface area of the microcapsule, allowing the composite matrix to better adhere to the microcapsule. Thus when the microcrack reaches the capsule it ruptures the microcapsules rather than causing it to debond from the matrix.

Brown et al. [23] studied the fracture mechanics issues that dealt with the development and optimization of self-healing composite materials, using the self-healing system developed by White et al. [81]. The crack healing efficiency, $\eta$, is defined as the ability of a healed sample to recover fracture toughness [84]. Brown et al. [23] and White et al. [81] used a tapered double-cantilever beam (TDCB) in their fracture experiments.

EPON® 828 epoxy resin and 12pph Anacmine ${ }^{\circledR}$ DETA curing agent were used to fabricate TDCB specimens [23]. The epoxy resin was mixed, then degassed and poured into a mold and cured for $24 \mathrm{hrs}$ at room temperature, followed by $24 \mathrm{hrs}$ at $30^{\circ} \mathrm{C}$. They varied the amounts of the catalyst and/or the microcapsules that were mixed into the resin. Their study was divided into the following three experimental configurations: 
1. Reference samples - epoxy with a range of microcapsule concentrations were tested to failure, then DCPD mixed with Grubbs' catalyst was manually inserted into the crack plane prior to crack closing.

2. Self-activated samples - epoxy with embedded catalyst were tested to failure and healed by the manual injection of DCPD into the crack plane.

3. In-situ samples - both the microencapsulated healing agent and Grubbs' catalyst were embedded in the specimens.

They tested the fracture specimens to failure, measuring the compliance and peak load. After the specimens were allowed to heal for 48 hrs they were retested and the healing efficiency was then calculated.

Four sets of samples were fabricated varying the catalyst to DCPD ratios. Ratios of 2, 4.4, 10 and $40 \mathrm{~g}$ liter-1 were used in the fabrication of 18 samples for each set. It was found that the healing efficiency increased as the concentration of the catalyst increased and the gel time decreased exponentially. Reference samples were also used to study the effects of varying the microcapsule concentration. Concentrations of $0-25$ wt $\%$ of $180 \mu \mathrm{m}$ diameter microcapsules were used in samples that were tested to failure and healed manually. Through the addition of solid particles the virgin fracture toughness of the material increased as the concentration of microcapsules increased. They claim that for a capsule concentration of $25 \mathrm{wt} \%$ and greater, near perfect healing was obtained.

Brown et al. [23] further report on the mixing order, catalyst particle size, and the catalyst concentration. Grubbs' catalyst retained activity when mixed with the EPON® 828/DETA system during cure. However, when mixed with the DETA curing agent 
alone the catalyst experienced rapid deactivation. To determine the best mixing sequence of the three components fracture samples were fabricated for each possible mixing sequence and then tested. The first two components were mixed for 5 min then degassed, the third component was then added and mixed for 5 min and degassed. It was found that mixing the catalyst and DETA curing agent first resulted in no measurable healing. Mixing the EPON® 828 with the DETA curing agent then the catalyst or the EPON® 828 with the catalyst then the DETA curing agent had little effect on the healing efficiency.

They then studied the catalyst particle size. The catalyst was ground to provide particle sizes ranging from $75 \mu \mathrm{m}$ to $1000 \mu \mathrm{m}$. These different particles sizes were then separated into four groups based on size and were used in the fabrication of samples. They found that catalyst particles of $180-355 \mu \mathrm{m}$ produced the highest healing efficiency. The catalyst concentration was then varied from $0 \mathrm{wt} \%$ to $4 \mathrm{wt} \%$ in samples in order to determine the concentration that provides a high healing efficiency without lowering the virgin fracture toughness. They found that the virgin fracture toughness decreased and the healing efficiency increased as the concentration of the catalyst increased.

In their investigation of the in-situ self-healing system $2.5 \mathrm{wt} \%$ Grubbs' Ru catalyst and $10 \mathrm{wt} \%$ of the microcapsules were used in the fabrication of the specimens. Specimens with microcapsule sizes of $180 \pm 40 \mu \mathrm{m}, 250 \pm 80 \mu \mathrm{m}$, and $460 \pm 80 \mu \mathrm{m}$ diameter were fabricated. The greatest healing efficiency was observed in specimens with the $460 \mu \mathrm{m}$ diameter microcapsules, which recovered $63 \%$ of the virgin load on average. The time for the reaction of Grubbs' catalyst and the DCPD healing agent also plays an important role in the healing efficiency of the specimen. 
Brown et al. [23] then fabricated specimens with $10 \mathrm{wt} \%$ of $180 \mathrm{~mm}$ diameter microcapsules and $2.5 \mathrm{wt} \%$ catalyst. Virgin fracture tests were preformed then healed fracture tests were preformed, at time intervals from $10 \mathrm{~min}$ to $72 \mathrm{~h}$ after the virgin test. They found that significant healing efficiencies developed after 25 min and steady-state values were reached after $10 \mathrm{hrs}$. They noticed that excess DCPD was present during fracture tests, regardless of the size of the microcapsules used. A reduction in concentration from $10 \mathrm{wt} \%$ to $5 \mathrm{wt} \%$ of the microcapsules had little impact on the healed fracture toughness.

In another study Brown et al. [24] investigated the toughening mechanisms induced by embedded microcapsules in a self-healing epoxy and the corresponding effects on the healing efficiency. They used a tapered double-cantilever beam (TDCB) test, following the previous study by White et al. [81] and using the self-healing system developed by White et al. [81]. Brown et al. retested the specimens after a $24 \mathrm{hr}$ period to determine the healed fracture toughness. Just like the other studies preformed at UIUC EPON® 828 epoxy resin and DETA curing agent were used to fabricate the TDCB specimens. The weight percentage of the microcapsule concentration was varied along with the diameter of the microcapsules $(50 \mu \mathrm{m}, 180 \mu \mathrm{m}$, and $460 \mu \mathrm{m})$. A 2.5 wt. $\%$ concentration of Grubbs' Ru catalyst was used for all specimens. In order to compare the fracture mechanisms, TDCB specimens were fabricated using silica microspheres and UF particles. Voids were also introduced into other samples by aggressive mixing of the resin before filling the mold.

The virgin fracture toughness of the TDCB specimens was tested as a function of microcapsule concentration. It was found that the fracture toughness increased with 
microcapsule concentration until reaching a peak for all three microcapsule sizes tested. They found the average maximum toughness to be $127 \%$ greater for neat epoxy containing $0 \%$ microcapsules. They concluded that specimens with smaller microcapsules exhibited maximum toughening at lower concentrations. Maximum healing efficiency for $180 \mu \mathrm{m}$ diameter microcapsules occurred at approximately $5 \mathrm{vol} \%$, while for $50 \mu \mathrm{m}$ diameter microcapsules, significant healing efficiency was measured at concentrations around $20 \mathrm{vol} \%$. One major factor in the recovery of material properties in the self-healing system is the weight percent of the embedded catalyst and microcapsules. Brown et al. [24] state that the healing efficiency is dependent on the microcapsule diameter. They argue that for smaller microcapsules a high healing efficiency only occurs at higher microcapsule concentrations since a larger number of microcapsules are required to deliver the same volume of DCPD healing agent into the fracture plane. By selecting the appropriate concentration and size of microcapsules Brown et al. were able to recover over $70 \%$ of the virgin fracture toughness.

Kessler and White [41] used the self-healing system developed at UIUC in woven composites to study the healing of delamination damage. The architecture of the reinforcement in woven composites makes them ideal for the use of the self-healing system. Resin rich areas in the woven composite are formed by the interlacing and undulating warp and fill yarns. They claim that a large number of the microcapsules can be stored in these resin rich areas and that they will have little effect changing the bulk material properties of the composite.

Just as with Brown et al. [23], Kessler and White used EPON® 828 and DETA as the matrix material. Eight-harness satin weave and plain weave E-glass fabric were used 
as the reinforcement fiber. These materials were used to fabricate double cantilever beam (DCB) specimens and were tested following ASTM D 5528-94a. They divided their study into three parts

1. To establish that the self-healing system is capable of significantly healing the composite. In order to test this they manually injected the catalyzed healing agent into the delamination region and then mechanically tested the specimen after the ROMP reaction was completed.

2. Their next step was to show that the embedded catalyst, Grubbs' Ru catalyst, was still active after the fabrication of the specimen. To test this they manually injected the uncatalyzed healing agent into the delamination region and then later tested the specimen.

3. Confirmation that the embedded microcapsules rupture during propagation of the delamination is needed. This was accomplished by examining the fracture surface after testing.

The final step was to combine all the aspects of the self-healing system into an in-situ system. Kessler and White only report on the completion of the first two steps mentioned above.

During fabrication of their specimens Grubbs' $\mathrm{Ru}$ catalyst was mixed into two locations of the specimens. In the center two fabric layers $1.75 \mathrm{wt} \%$ of catalyst was used. 0.17 wt. $\%$ or $0.33 \mathrm{wt} . \%$ was dispersed in the area that extended $75 \mathrm{~mm}$ beyond the mid-plane of the specimen. They measured the fiber volume fraction of the specimens to be $27 \%$ and $29 \%$ for the plain weave and satin weave specimens, respectively. 
They found that all of the specimens exhibited interfacial debonding as the dominant mode of failure. After examining the fracture plane of the self-activated specimens they noticed that the coverage of the polymerized healing agent was incomplete. They conclude that the rate of in-situ polymerization for self-activated materials must be fast to prevent diffusion of the monomer into the matrix. By manually injecting the uncatalyzed healing agent into the fracture plane a healing efficiency of $20 \%$ was achieved. A 51-67\% healing efficiency was obtained for specimens where the catalyzed healing agent was manually injected into the fracture plane. They further showed that since the healing system is a living polymerization [42], repeated healings could occur.

Kessler et al. [44] studied the repair of delamination damage in width-tapered double cantilever beam, WTDCB, specimens. They used microcapsules with a mean diameter of $166 \mu \mathrm{m}$ that contain the healing agent dicyclopentadiene. Grubbs' Ru catalyst was used to trigger a ROMP reaction with the DCPD to produce a healing polymer. EPON®R 828 and a tertiary amine system (Ancamine K54) along with carbon fiber were used to fabricate the composite specimens. Heloxy 71, a high molecular weight epoxy flexiblizer, was added to the matrix. The Heloxy 71 was used to improve the toughness of the matrix and to improve subsequent crack growth stability. The composite specimens were fabricated using hand lay-up and compression molding. The EPON® 828 was mixed with Heloxy 71 at a concentration of $5: 3$ by weight. Ancamine K54 was then added at a concentration of 10:100 by weight. Grubbs' Ru catalyst was ground with a mortar and pestle and mixed into a portion of the resin at $5 \mathrm{wt} \%$. The resin containing the catalyst was used to impregnate the central four fabric layers. For 
self-healing samples microcapsules were mixed with the resin at $20 \mathrm{wt} \%$ concentration and then used to impregnate the central fabric layers.

For their study three types of specimens were manufactured: reference and selfactivated specimens that serve as experimental controls and self-healing specimens. The catalyst and the healing agent are manually injected into the delamination region of the reference specimens. For the self-activated specimens the catalyst is embedded in the matrix and the healing agent is manually injected. Self-healing specimens contain the catalyst and the microcapsules, which are embedded in the matrix and no manual injection is required. The self-healing specimens were tested at different time intervals of healing from 10 min to $48 \mathrm{hrs}$. Self-healing specimens were also broken into two groups one that was healed at room temperature and the other at $80^{\circ} \mathrm{C}$.

The average healing efficiency for the reference specimens was $\eta_{\text {avg }}=99 \%$ and the maximum healing efficiency was $\eta_{\max }=107 \%$. For the self-activated specimens the average healing efficiency was $\eta_{\text {avg }}=73 \%$ and $\eta_{\max }=82 \%$. The average healing efficiency for the self-healing specimens at room temperature was $\eta_{\text {avg }}=38 \%$ and $\eta_{\max }=$ $45 \%$. At $80^{\circ} \mathrm{C}$ the average healing efficiency for the self-healing specimens was $\eta_{\text {avg }}=$ $66 \%$ and $\eta_{\max }=80 \%$. Kessler et al. [44] observed no measurable healing in the specimens until $t_{\text {heal }}>30 \mathrm{~min}$, which they found to correlate closely to the gel time of the shelf-healing system at room temperature. They found that the healing efficiency increased with the time the specimen was allowed to heal until a maximum efficiency was reached at $48 \mathrm{hrs}$.

Rule et al. [71] used paraffin wax to encapsulate Grubbs' catalyst in an effort to make lower catalyst loadings more effective. They found areas where using an 
encapsulated catalyst is beneficial. First, they found that the catalyst does not disperse well in the epoxy matrix. Secondly, they found evidence that the epoxy's curing agent, diethylenetriamine (DETA), destructively attacks Grubbs' catalyst as the epoxy initially cures. These two factors reduce the overall healing efficiency of the system. The healing effect of the epoxy reduces the amount of catalyst that is available and the poor dispersion characteristic limits the amount of catalyst that can come into contact with the healing agent, DCPD. There solution to solve these problems is to encapsulate Grubbs' catalyst with paraffin wax. This process is outlined in Chapter 2. Fracture testing of tapered double-cantilever beam specimens containing lower concentrations of the waxprotected catalyst produced healing efficiencies that are greater than those previously reported. They varied the amount of the catalyst in the microspheres from 0 wt.- $\%$ to 1.25 wt.- $\%$. They found that the healing efficiency increases as the catalyst loading increases and appears to level off around $0.75 \mathrm{wt} .-\%$. At $0.75 \mathrm{wt} .-\%$ catalyst a healing efficiency of $93 \%$ was observed.

\subsubsection{Damage Modeling of Composite Materials}

Ladeveze and Dantec [50] modeled composite laminate damage at the elementary-ply scale. They used the continuum damage mechanics theory to describe matrix microcracking and fiber/matrix debonding. In their work, they assumed a planestress state and only take into account in-plane strains. They used a plasticity model to model the inelastic strains induced by damage which are based on the effective stress and the effective plastic strain. The model distinguishes two ply-degradation mechanisms that contribute to damage development - matrix microcracking and fiber matrix debonding. Only cases with minor delamination effects were considered in the study. 
T300/914 and IM6/914 carbon-fiber-reinforced epoxy composites were used in the study.

For quasi-static cases they felt that comparisons with experimental testing and their model were very satisfactory.

Zhu and Cescotto [93] developed an energy-based damage model at finite strains for ductile fracture. Their work extends the energy based anisotropic damage model proposed by Cordebois and Sidoroff $(1979,1982)$ and Cordebois $(1983)$. They take into account anisotropic elasticity, anisotropic plasticity, and anisotropic damage. They used the damage effect tensor, M, originally proposed by Chow and Wang (1987). They introduced a damage characteristic tensor, J, which is based on the hypothesis of damage energy equivalence. A two-step operator split algorithm (elastic predictor and coupled plastic-damage corrector) with sub increments was developed to integrate fully coupled elastoplastic damage constitutive relations. They used their model to simulate hemispherical punch stretching including heat transfer and deep drawing by cylindrical and square punches.

Voyiadjis and Park [87, 88] proposed a model that incorporates damage and plastic deformation in the analysis of fiber-reinforced metal matrix composites. They modeled constitutive damage relations for the matrix and the fiber. Then, these are coupled with the interfacial damage between the matrix and the fiber. A von Mises type yield criterion with an associated flow rule and a Ziegler-Prager kinematic hardening rule are used for the undamaged matrix material. Their model achieved good correlation between experimental and numerical results of laminate lay-ups of $[0 / 90]_{s}$ and $[ \pm 45]_{s}$.

Voyiadjis and Deliktas [89] introduced rate-independent and rate-dependent models to describe the coupled incremental damage and plasticity theory for composite 
materials. They incorporated damage and inelastic deformations in their model for the analysis of fiber-reinforced metal-matrix composite materials. Damage is described in the elastic and inelastic domains using a fourth-order damaged effect tensor which is a function of a second-order damage tensor. Their model uses the Newton-Rapson iterative scheme for the overall laminate analysis. To validate their model Voyiadjis and Deliktas tested laminates of $[0]_{8 s},[90]_{8 s},[0 / 90]_{4 s}$, and $[ \pm 45]_{2 s}$ at room temperature, $538^{\circ} \mathrm{C}$ and $649^{\circ} \mathrm{C}$. Good correlations between the numerical model and uniaxial tension experimental results of titanium metal-matrix composites reinforced with silicon carbide fibers for the lay-ups mentioned above were achieved.

Several studies support the Continuum Damage Model proposed by Barbero et al. $[14,16,17,18,19,75]$. Laminates fabricated with Aramid/epoxy, T300/5208, T300/914 carbon/epoxy, and plain weave composites have been used to validate the damage model. The CDM model forces the damage surface to match the Tsai-Wu failure criterion at failure. Thus, the ultimate failure of a lamina is as accurate as the Tsai-Wu failure criterion [16]. One of the main points of the CDM model is to compare the Tsai-Wu with the damage domain in the effective stress space, obtaining a linear system [18]. Several internal material constants that are related to the experimental material properties are used in the CDM model. A fourth order tensor, J, and a second order tensor, $\mathrm{H}$, are used to define the damage domain. The $\mathrm{J}$ and $\mathrm{H}$ tensors are used to control the shape of the damage and potential surfaces. The damage threshold is defined as $\gamma_{\mathrm{o}}$ and the damage evolution parameters are defined as $c_{1}^{d}$ and $c_{1}^{h}$. Each of these three parameters are calculated by adjusting the shear stress-strain curve obtained from finite element analysis of a unidirectional ply subjected to pure shear conditions to match the experimental shear 
stress-strain data. Their CDM model uses an incremental-iterative procedure based on a return-mapping algorithm, predictor-corrector scheme.

Barbero and Lonetti [16] used the CDM model to predict the behavior of an offaxis test for Aramid/epoxy and several off-axis tests for T300/5208 and T300/914 carbon/epoxy. In [17] Barbero and Lonetti use the CDM model to successfully predict the monotonic loading behavior of a $[45 /-45]_{2 s}$ carbon/epoxy T300/915 laminate. They also used the model to predict the monotonic loading behavior of a $10^{\circ}$ off-axis Aramid/epoxy lamina. Barbero et al. [18] used the model in the analysis of the interlaminar damage of three different types of composite laminates under torque loading conditions. In [75] Sikkil used the CDM model to predict the non-linear behavior of plain weave laminates under tensile loading. He validated the model for the tensile response of a T300/5208 laminate for the following configurations [10/-10] $]_{2 s}$, $[0 / 45 /-$ $45 / 90]_{s},[30 /-30]_{2 s}$, and $[45 /-45]_{2 s}$. Lastly, he analyzed the damage behavior of iso-phase and out-of-phase plain weave fabrics.

\subsubsection{Composite Material Testing}

The material parameters used in the CDM model are $E_{1}, E_{2}=E_{3}, G_{12}=G_{13}, G_{23}$, $v_{12}=v_{13}, v_{23}, F_{1 t}, F_{1 c}, F_{2 t}, F_{2 c}, F_{4}, F_{5}, F_{6}, G_{12}$ damaged, $G_{13}$ damaged, and $G_{23}$ damaged [18]. By looking at these required parameters a quick assessment of the tests required to obtain these properties was done. Compression, shear, and tensile testing are all required to obtain these parameters. This section discusses various testing methods for the parameters mentioned above. 


\subsubsection{Compression Test}

Wen [80] found through experimental studies of Cytech Fiberite 949 HYE/M30GC and 948A1 HYE/M40J both of which are prepreg carbon/epoxy materials that for compression test the SACMA SRM-1R-94 [72] procedure provides better test results when compared to the ASTM D3410 "IITRI" [8] test. He states that the ASTM D3410 "IITRI" test gave low values of compression strength. The SACMA test typically provided a compressive strength of $5 \%$ better than the D3410 "IITRI" method. It is believed that this was due to the end loading of the SACMA specimens. The ASTM D 3410/D 3410M- 95 loads the specimen in compression by a shear load acting along the grips. A wedge is used to apply the shear load in a specially designed fixture.

\subsubsection{Shear Test}

The ASTM D5379 [10] "Iosipescu" method was used by Wen [80] to test for the shear properties of Cytech Fiberite 949 HYE/M30GC and 948A1 HYE/M40J both of which are prepreg carbon/epoxy materials. ASTM D5379 uses a material coupon in the form of a rectangular flat strip with symmetrical centrally located v-notches. A special testing fixture is used to load the specimen with the line of action coinciding with the notch. Two strain gages, oriented at $\pm 45^{\circ}$ to the loading axis, are placed in the middle of the specimen. It has been found that when testing in the 1-2 plane the best results have been obtained using [0/90]ns laminates [10]. Zhou et al. [92] also used the Iosipescu fixture to test for the in-plane and interlaminar shear properties of three different lay-ups of carbon/epoxy. Based on results from [32] Zhou et al. choose not worry about the effects of out-of-plane torsion and did not place strain gages on both sides of the specimen as suggested $[60,66]$. Their stress and moduli results showed little scatter. 
They believe that the validity of the in-plane shear tests of quasi-isotropic lay-up and unidirectional lay-up in the 2-3 shear plane are questionable due to undesired failure modes. Another test method for the in-plane shear response of polymer matrix composite materials is the ASTM D 3518/D 3518M [9] test. This test is a uniaxial tension test of a $\pm 45^{\circ}$ laminate following the ASTM D 3039 test method, discussed in the following section, for tensile properties.

Davalos et al. [28] used classical macromechanics and modified transverse shear deformation theory to predict the shear stiffness properties. They evaluated the shear moduli for FRP structural laminates from torsion tests using paired samples with material orientations normal to each other. Data-reduction methods from solutions by Leknitskii [51] and Whitney [83] of torsion test are used. Davalos et al. state that the major disadvantages of the Iosipescu test are the small effective test area and the stress concentration at the notched section of the specimen. They claim that the torsion test produces a pure shear state of stress in the specimen.

\subsubsection{Tensile Test}

ASTM D 3039/D 3039 M [7] and SACMA SRM 4R-94 [73] are standard test methods to determine the tensile properties of polymer matrix composite materials. The SACMA SRM 4R-94 standard is a derivation of the ASTM 3039 standard and is basically the same test standard. Both standards test specimens measuring 0.04 " $\mathrm{x} 0.5$ " $\mathrm{x}$ 10 " for $0^{\circ}$ laminates and $0.08 " \mathrm{x} 0.5$ " x 10 " for $90^{\circ}$ laminates. Each method tests for the ultimate tensile strength, and strain, the tensile chord modulus of elasticity, and Poisson's ratio. 


\subsubsection{Composite Fabrication Methods}

Several fabrication methods of composite materials exist. The main factors that influence the decision to choose a particular method are the type of matrix and fibers, cost of the fabrication method, and the temperature and cure time of the matrix. Depending on the part that is being fabricated a mold may be required. A mold is a mirror image of the part that is being produced. In some instances the fabrication of the mold can be as tedious as the fabrication of the part itself. The surface texture and shape of the mold are crucial in the products final outcome. The following is a list of some of the more popular fabrication techniques

- Hand lay-up

- Prepreg lay-up

- Vacuum bag molding

- Autoclave processing

- Compression molding

- Resin transfer molding

- Pultrusion

- Filament winding

A detailed description of each of these methods, their advantages and disadvantages can be found in Barbero 1999 [13] and Herakovich 1998 [35].

\subsection{Scope of Project}

The goals of this project are:

1. Fabricate microcapsules in the same manner outlined by White et al. [81]. 
2. Conduct tests on specimens with microcapsules and catalyst to obtain measurable healing effects.

3. Improve upon the healing portion of the CDHM model described in [19].

4. Propose and conduct tests to identify the healing parameters outlined in the improved CDHM model only in the 1-2 direction.

5. Implement the model in ANSYS.

6. Validate the model by performing additional tests not used in the parameter identification. 

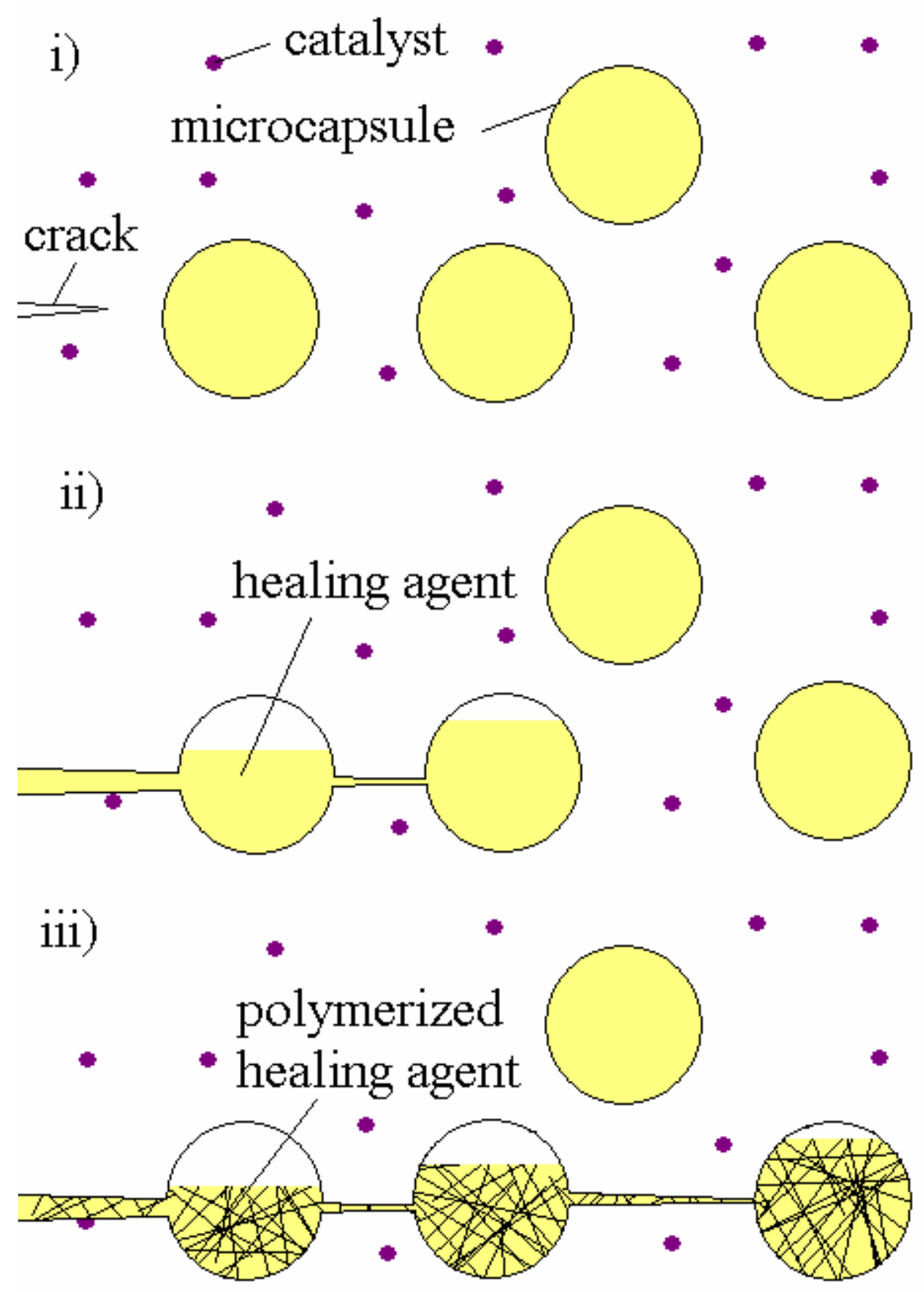

Figure 1.1 Self-healing concept 


\section{SELF-HEALING CONCEPT}

This chapter discusses the in situ poly(urea-formaldehyde) microencapsulation process of dicyclopentadiene process and modifications of this process outlined by Brown et al. [22], Kessler [43], and White et al. [81]. Even though each of the references contains research done at UIUC, there seems to be slight differences in the process each reference uses to fabricate the microcapsules. Kessler's work was assumed to be the newest and most complete body of literature dealing with the development and fabrication of the microcapsules. This chapter further discusses the wax encapsulation process of Grubbs' Ru catalyst outlined by Rule et al. [71].

\subsection{Materials and Equipment}

Ethylene maleic anhydride (EMA) copolymer was obtained from Zeeland Chemicals. Dicyclopentadiene (DCPD), urea, ammonium chloride, formaldehyde, and sodium hydroxide were purchased from Fisher Scientific. Resorcinol, hydrochloric acid, and 1-octanol were purchased from J.T. Baker. K-type thermocouples and thermocouple reader were purchased from OMEGA. A Eurostar power control-visc digital mixer was purchased from IKA Works, INC. A three-bladed, 63.5mm diameter low-shear mixing impeller was purchased from Cole Parmer. All solvents and substance used for preparation of EMA solution, acid and base solutions and 1-octanol were of analytical grade. Bis(tricyclohexylphosphine)benzylidine ruthenium (IV) dichloride (Grubbs' Ru catalyst) was purchased from Materia. A Gilson Performer III sieve shaker and sieves

were purchased from Gilson Company, Inc. Neutral activated aluminum oxide and paraffin wax was purchased from Sigma Aldrich. 


\subsection{Fabrication of Microcapsules}

In situ polymerization of the microcapsules takes place in an oil-in-water emulsion as outlined in Figure 2.1. First, $200 \mathrm{ml}$ of deionized water is poured in a 1000 $\mathrm{ml}$ beaker and brought to and held at $21^{\circ} \mathrm{C}$ to $23^{\circ} \mathrm{C}$. The beaker is suspended in a water bath on a hotplate with a magnetic stirrer, Figure 2.2. The temperature of the water bath and the solution is monitored using K-type thermocouples (Omega). A digital mixer driving a three-bladed impeller is then used to agitate the solution at the desired rpm (agitation rate). Brown et al. [22] have shown that the size of the microcapsule is dependent on the agitation rate of the emulsion. The agitation rate controls the size of the emulsification droplet, in our case DCPD. Brown et al. [22] further showed that a linear relation exists between the $\log ($ mean diameter $)$ and $\log ($ agitation rate $)$. Beaker diameter, height of the impeller from the bottom of the beaker, and impeller size each contribute to the size of the capsule and the size distribution of the capsules.

Next, $50 \mathrm{ml}$ of $2.5 \mathrm{wt} \%$ aqueous solution of EMA copolymer, $5.00 \mathrm{~g}$ urea, $0.50 \mathrm{~g}$ ammonium chloride, and $0.50 \mathrm{~g}$ resorcinol are mixed into the emulsion. The EMA solution needs to be prepared in advance because it takes several hours to dissolve under constant mixing. EMA solution is used to reduce the viscosity of the aqueous medium and avoids the need to dilute the emulsion to avoid nugget formation [43].

Next, the $\mathrm{pH}$ is raised to 3.75 by drop-wise addition of sodium hydroxide $(\mathrm{NaOH})$. Then the $\mathrm{pH}$ is lowered to 3.50 by drop-wise addition of hydrochloric acid $(\mathrm{HCl})$. A few drops of 1-octanol are then added to the solution to remove surface bubbles. $60 \mathrm{ml}$ of distilled DCPD are then added by a steady stream to the solution and 
permitted to stabilize for approximately 10 minutes. $12.67 \mathrm{~g}$ of $37 \mathrm{wt} \%$ aqueous solution of formaldehyde is then added to obtain a 1:1.9 molar ratio of formaldehyde to urea.

The emulsion is then covered and brought to $55^{\circ} \mathrm{C}$ by turning up the heat on the hotplate at a rate of $1^{\circ} \mathrm{C} / \mathrm{min}$, Figure 2.3. After two hours of mixing at temperature $50 \mathrm{ml}$ of deionized water is then added to replace evaporated water. Then after four hours of agitation at temperature the hot plate and mixer are turned off and the emulsion is allowed to cool to room temperature. The microcapsule slurry is then vacuum filtered as shown in Figure 2.4 and rinsed with deionized water. The slurry is then dried using the vacuum filter to pull air through the caked microcapsules (Figure 2.5). Next, the dry, clumped microcapsules are placed in a sieve shaker, Figure 2.6, purchased from Gilson Company Inc. in order to break up the clumps of capsules and to separate the powder by diameter. Sieve trays of $20 \mu \mathrm{m}, 38 \mu \mathrm{m}, 53 \mu \mathrm{m}, 75 \mu \mathrm{m}, 106 \mu \mathrm{m}, 150 \mu \mathrm{m} 212 \mu \mathrm{m}$, and $250 \mu \mathrm{m}$ were used to determine the size distribution of the capsules.

\subsection{Distillation of DCPD}

Kessler [43] discusses the need to distill the DCPD before using it in the microencapsulation process. The inhibitor p-tert-butylcatechol is present in the DCPD. This inhibitor prevents the DCPD from polymerizing in the bottle, it also prevents the urea and formaldehyde from reacting around the droplets of DCPD in the emulsion. Instead of forming a shell around the DCPD, the urea-formaldehyde reaction takes placeforming clumps in the emulsion (Figure 2.7).

The distillation set-up is shown in Figure 2.8. DCPD and neutral activated aluminum oxide (Aldrich Chemical Company Inc.) are placed in a round bottom flask and immersed in a silicon bath. The distillation is performed in a partial vacuum. The oil 
bath is slowly heated until evaporation of the DCPD begins, while the round bottom flask containing the DCPD and activated aluminum oxide is slowly rotated. Kessler calls the first $10-20 \%$ of the distilled product the "first fraction" and collected it in a beaker and discards it after the distillation. It is thought that the first fraction contains some lower molecular-weight components such as cyclopentadiene. Kessler also discards the last 5$15 \%$ of the undistilled DCPD. The remaining, distilled, DCPD is then placed in a beaker and stored in a cold room in an attempt to keep it from polymerizing.

\subsection{Crush Strength of Microcapsules}

The crush strength of the microcapsules is tested to determine a relationship between the microcapsule diameter and the strength of the microcapsule shell. This is useful when determining the microcapsule size to place in the composite laminate. Another aspect where this could be used is in the case of multiple or staged healings of a composite material. This would allow one to stagger the rupturing of microcapsules under different loading conditions.

Microcapsules are first sifted into sieves of $20 \mu \mathrm{m}, 32 \mu \mathrm{m}, 53 \mu \mathrm{m}, 75 \mu \mathrm{m}, 106$ $\mu \mathrm{m}, 150 \mu \mathrm{m}, 212 \mu \mathrm{m}$, and $250 \mu \mathrm{m}$. The crush strength of the capsules is tested using an Instron testing machine, Figure 2.9. First, 1 to 2.5 grams of microcapsules are placed in the compression test apparatus, Figure 2.10. The volume of the microcapsules are then measured and recorded. The compression test apparatus is then placed in the Instron. A load is then applied until the crosshead has moved slightly less than the height of the microcapsules in test apparatus.

Software that was supplied with the Instron is setup to calculate the stress and strain. The stress is calculated using the following equation 


$$
\sigma=\frac{P}{a}
$$

where $\mathrm{a}$ is the area of the die and $\mathrm{P}$ is the load. The compressive strain percentage is calculated using the following equation

$$
\mathcal{E}=100 \frac{\delta}{L_{o}}
$$

where $\delta$ is the change in height of the microcapsules and $L_{o}$ is the original height of the microcapsules in the testing apparatus. Microcapsules with diameters of $212 \mu \mathrm{m}, 150$ $\mu \mathrm{m}, 106 \mu \mathrm{m}$, and $53 \mu \mathrm{m}$ were tested to determine the crush strength of the respective microcapsule. Figure 2.11 is a plot of the stress vs. strain $\%$ for each of the microcapsule sizes tested. An arrow marks the inflection point where the shell wall ruptured. The data that follows the inflection point represents the urea-formaldehyde shell fragments as they are being compressed. The stress marked by the inflection point does not represent the crush strength of an individual microcapsule instead it represents the crush strength of the whole quantity of microcapsules that were in the compression test apparatus.

In order to determine the crush strength of an individual microcapsule the number of microcapsules tested needs to be determined. To do this the volume of the microcapsules in the compression apparatus is calculated by multiplying the height of the microcapsules by the area of the die of testing apparatus. Since the microcapsules are assumed to be perfect spheres a face-centered cubic closed packed plane is assumed. The atomic packing factor, APF, of a face-centered cubic closed packed plane is 0.74 [25]. This means that the fraction of solid sphere volume in a unit cell is 0.74 . Therefore the volume occupied by the microcapsules is $0.74 \%$ of the measured volume. Knowing the diameter of the microcapsules and the volume occupied in the testing apparatus, the 
number of microcapsules used during the test can then be calculated. The crush strength, determined from Figure 2.12 through Figure 2.15, was then divided by the number of microcapsules tested. Table 2.1 lists the microcapsule diameter, volume, mass, and number of microcapsules tested, and the crush strength per number of microcapsules. Figure 2.16 is a graph of the crush strength as a function of microcapsule diameter. From Figure 2.16 one can determine that a linear relationship exists between the diameter of the microcapsules and the respective crush strength. One can also see that as the diameter of the microcapsules increase the force required to crush the shell wall decreases.

\subsection{Wax Encapsulation of Grubbs' Ru Catalyst}

The wax encapsulation process of Grubbs' Ru catalyst developed by Rule et al. [71] is outlined in this section. In a $\mathrm{N}_{2}$-filled glove box $10.0 \mathrm{~g}$ of paraffin wax and 525 mg of Grubbs' Ru catalyst are sealed in a vial. Unlike the method outlined by Rule et al., the catalyst was not ground into uniform size particles. Next, $225 \mathrm{ml}$ of water, $0.63 \mathrm{~g}$ (0.28 wt.- $\%)$ of poly(ethylene-co-maleic anhydride), and 1 drop of octanol are placed in a $1000 \mathrm{ml}$ beaker in an $82{ }^{\circ} \mathrm{C}$ water bath and stirred with a mechanical stirrer at $900 \mathrm{rpm}$. The vial is taken out of the glove box and submerged in the $82{ }^{\circ} \mathrm{C}$ water bath. After 10 min the wax melts and the aqueous solution reaches $65-70{ }^{\circ} \mathrm{C}$. The vial is then shaken to disperse the catalyst and is then opened and the wax and catalyst is poured into the aqueous solution. After $2 \mathrm{~min}, 600 \mathrm{ml}, 0{ }^{\circ} \mathrm{C}$ water (ice water) is added, the stirring is stopped, and the solution is quickly removed from the hotplate. The microspheres are then collected by filtration and dried under a vacuum. 


\subsection{Results}

First attempts at fabricating microcapsules containing DCPD were unsuccessful. Based on the work by [22] it was unclear why and how to distill the DCPD. It was thought that 95\% pure DCPD would be sufficient enough to polymerize with Grubbs' catalyst. Results from the fabrication using off the shelf DCPD produced ureaformaldehyde clumps. As a test to see if distilling the DCPD was necessary, mineral oil was then substituted into the emulsion and was successfully encapsulated. The process for distilling the DCPD is outlined in [43].

Another setback was determining the value to raise the $\mathrm{pH}$ of the emulsion to when adding the sodium hydroxide. Based on Kessler's work [43] the pH is supposed to be raised slightly above 3.5 through drop-wise addition of $\mathrm{NaOH}$, then lowered to 3.5 by the addition of $\mathrm{HCl}$. A pH of 3.75 was settled on as a value slightly higher than 3.5 and when used the encapsulation of DCPD was successful.

Figure 2.14 is the size distribution obtained from microcapsules batches at agitation rates of $550 \mathrm{rpm}, 850 \mathrm{rpm}$, and $1150 \mathrm{rpm}$. As expected from the work done at UIUC as the agitation rate increases the diameter of the microcapsules decreases. The goal of this research is not to study the microcapsules; instead it is just to fabricate them. Therefore, microcapsules were not fabricated at several different agitation rates in an effort to reproduce data that would give an equation relating agitation rate to microcapsule diameter.

A scanning electron microscope was used to look at the microcapsules. In Figure 2.18 one notices microcapsules of several different diameters along with broken microcapsules and broken microcapsule shells. Transferring of the microcapsules 
between containers and the vacuum filtration process is thought to be the cause of the broken capsules. Figure 2.19a and Figure 2.19b are pictures of a microcapsules that are approximately $200 \mu \mathrm{m}$ in diameter. Notice the rough outer surface of the microcapsule shell due to the left over urea-formaldehyde adhering to the shell wall. In Figure 2.20 one can notice the urea-formaldehyde debris easier. Figure 2.21 is a broken microcapsule notice the inner surface is smooth, compared to the rough outer surface. In Figure 2.22 one can see the shell wall of the microcapsule which measures approximately $0.30 \mu \mathrm{m}$ thick.

Once these obstacles were overcome the process of fabricating DCPD filled microcapsules was not that difficult. As stated by Kessler [43], diluting the EMA copolymer does take several hours to complete. The easiest way to make the dilution is to mix the EMA copolymer and de-ionized water in a beaker and let it sit overnight. The procedure for the fabrication of the microcapsules is outlined in Appendix A and a comparison of the different encapsulation methods in the literature can be found in Appendix B.

Further crush strength testing needs to be done. The data presented here is only one set of data. Most testing standards require at least 5 specimens to be tested for accurate results. Additional crush test were not preformed because the Instron used for the crush strength testing is not in a hood and the DCPD has a strong odor. 
Table 2.1 Compression data from microcapsule testing

\begin{tabular}{|l|c|c|c|c|}
\hline Diameter $(\mu \mathrm{m})$ & 53 & 106 & 150 & 212 \\
\hline Volume tested $\left(\mathbf{m m}^{\mathbf{3}}\right)$ & 3745 & 6062 & 10425 & 3848 \\
\hline Mass tested $\mathbf{( g )}$ & 1.47 & 2.26 & 2.33 & 1.00 \\
\hline Number of microcapsules tested & $5.56 . \mathrm{E}+05$ & $8.99 . \mathrm{E}+05$ & $1.55 . \mathrm{E}+06$ & $5.71 . \mathrm{E}+05$ \\
\hline Crush strength $(\mathbf{P a})$ & $9.08 . \mathrm{E}-01$ & $6.64 . \mathrm{E}-01$ & $4.45 . \mathrm{E}-01$ & $1.21 . \mathrm{E}-01$ \\
\hline
\end{tabular}




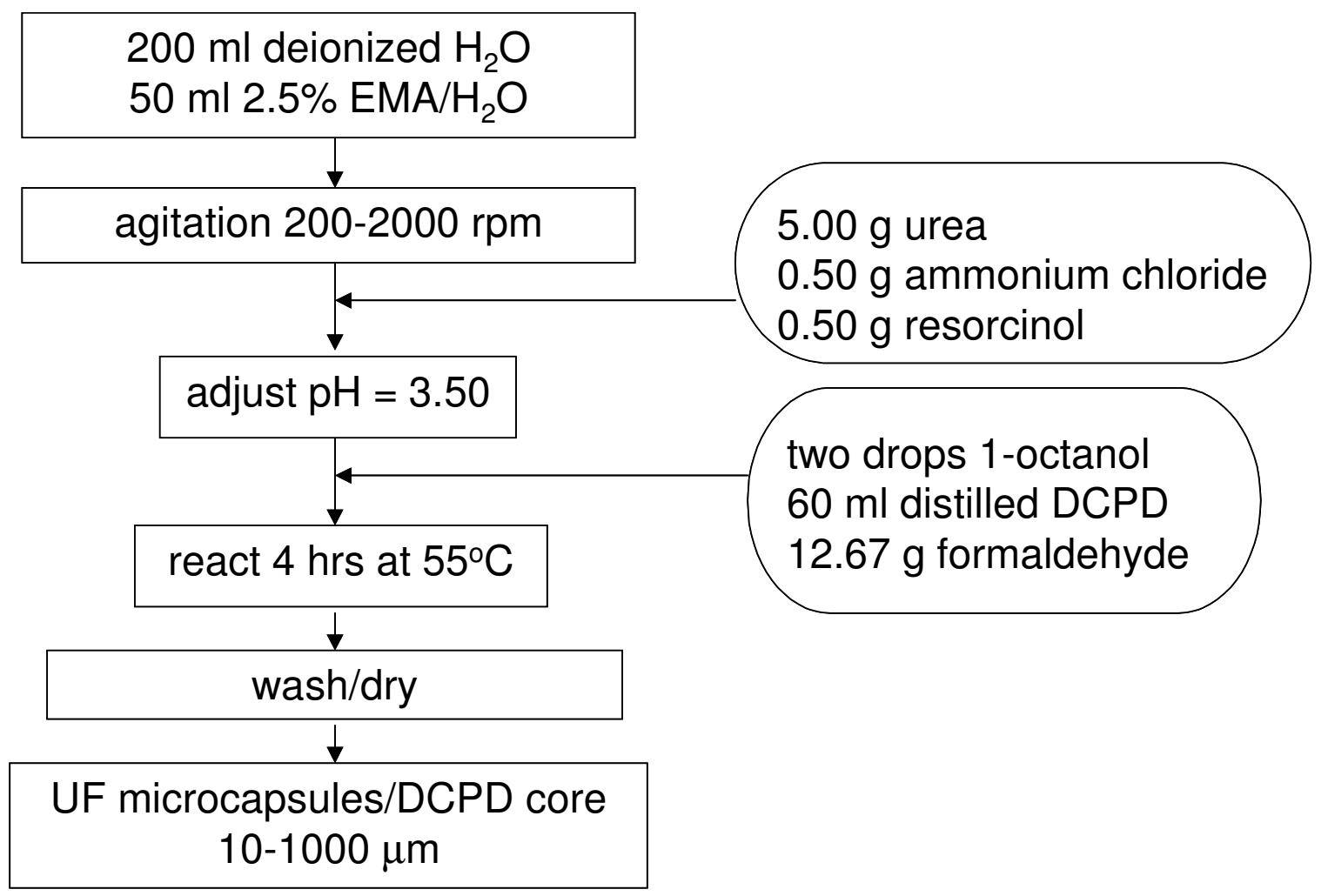

Figure 2.1 Flowchart outlining the fabrication process of microcapsules. 


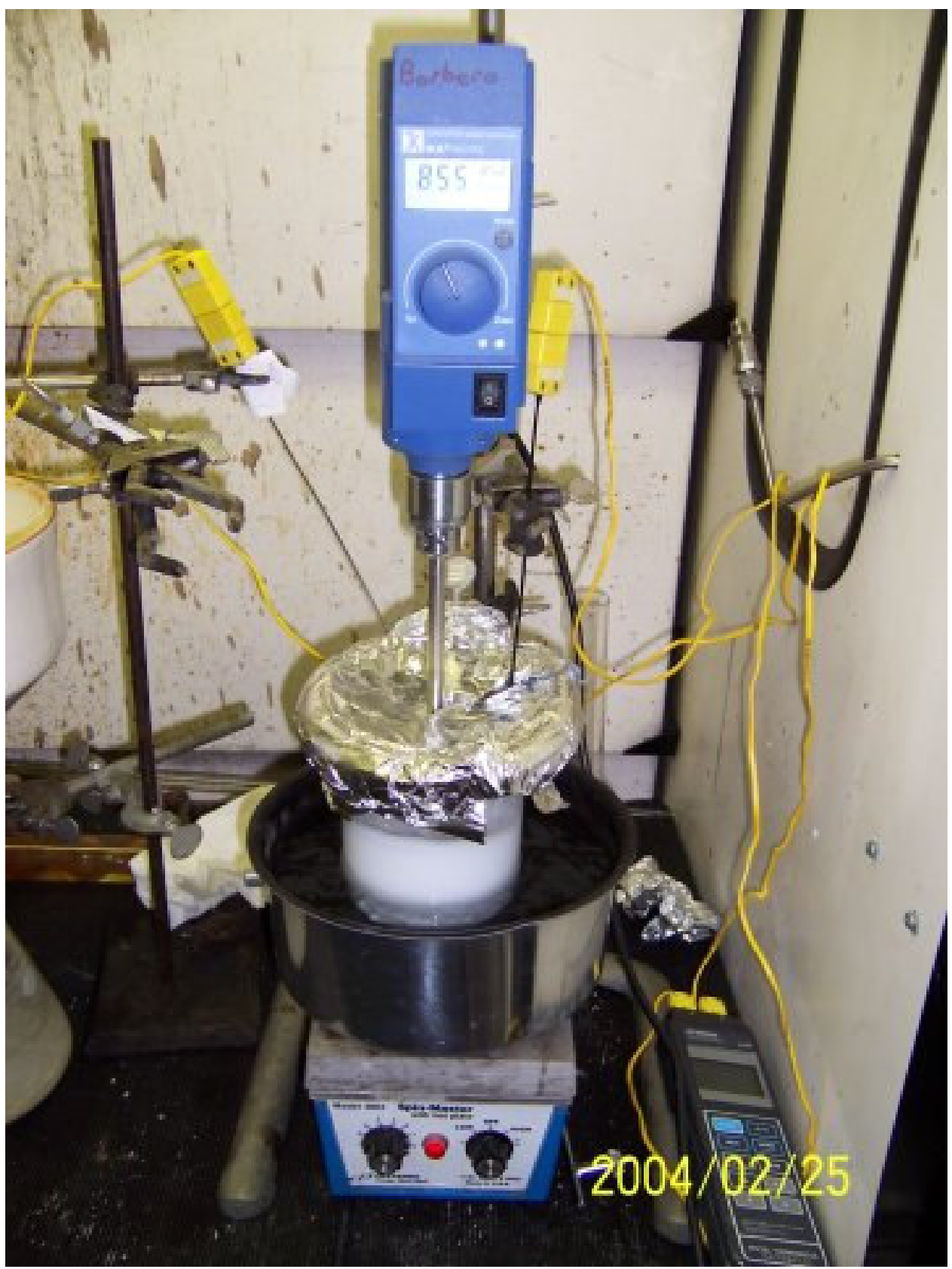

Figure 2.2 Experimental setup used in the fabrication of the microcapsules. 


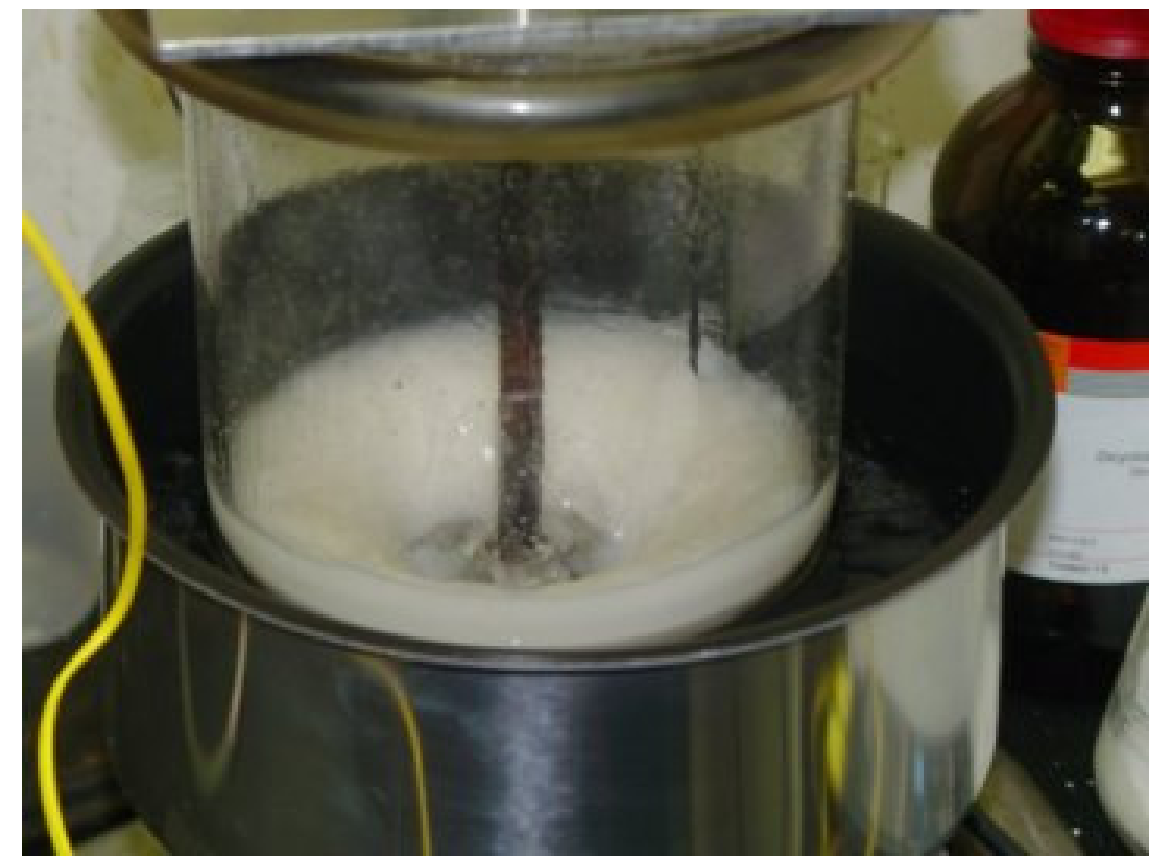

Figure 2.3 Close-up of oil-in-water emulsion during the microencapsulation process.

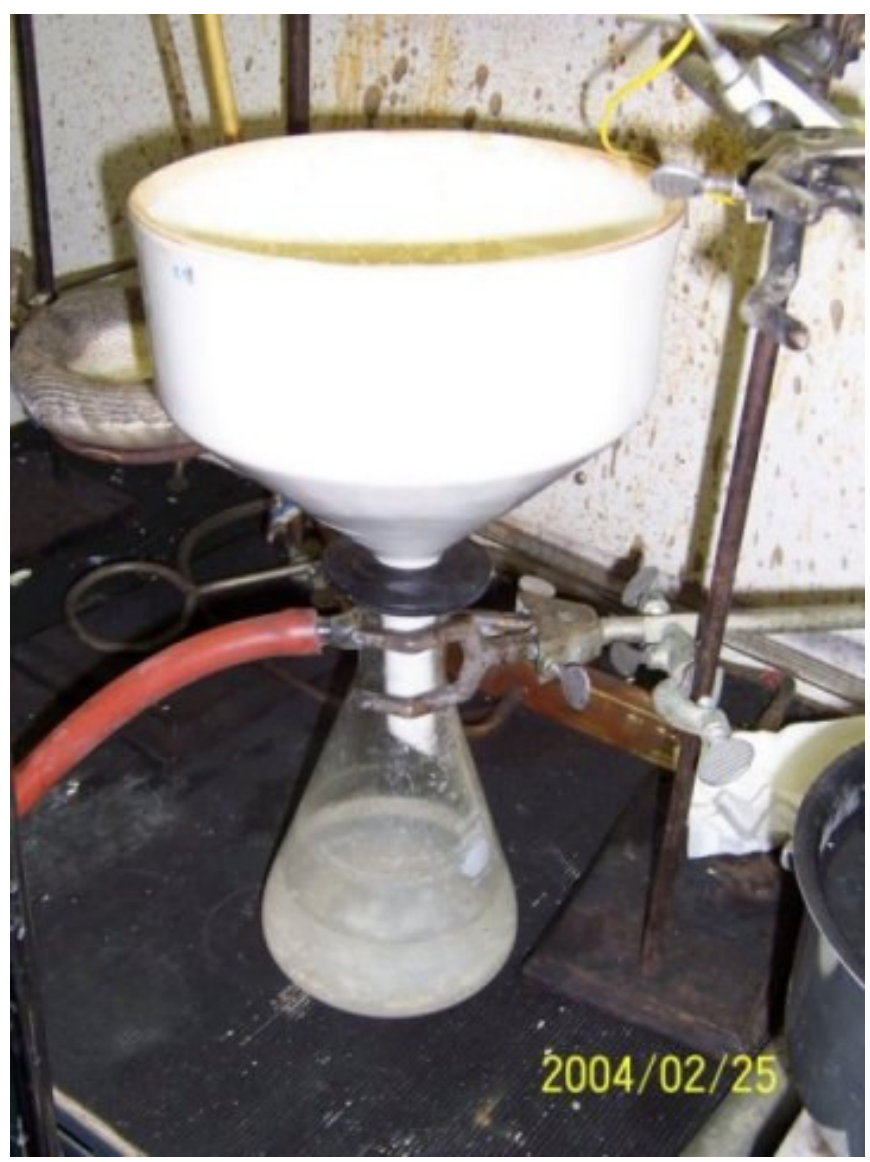

Figure 2.4 Vacuum filtration system. 


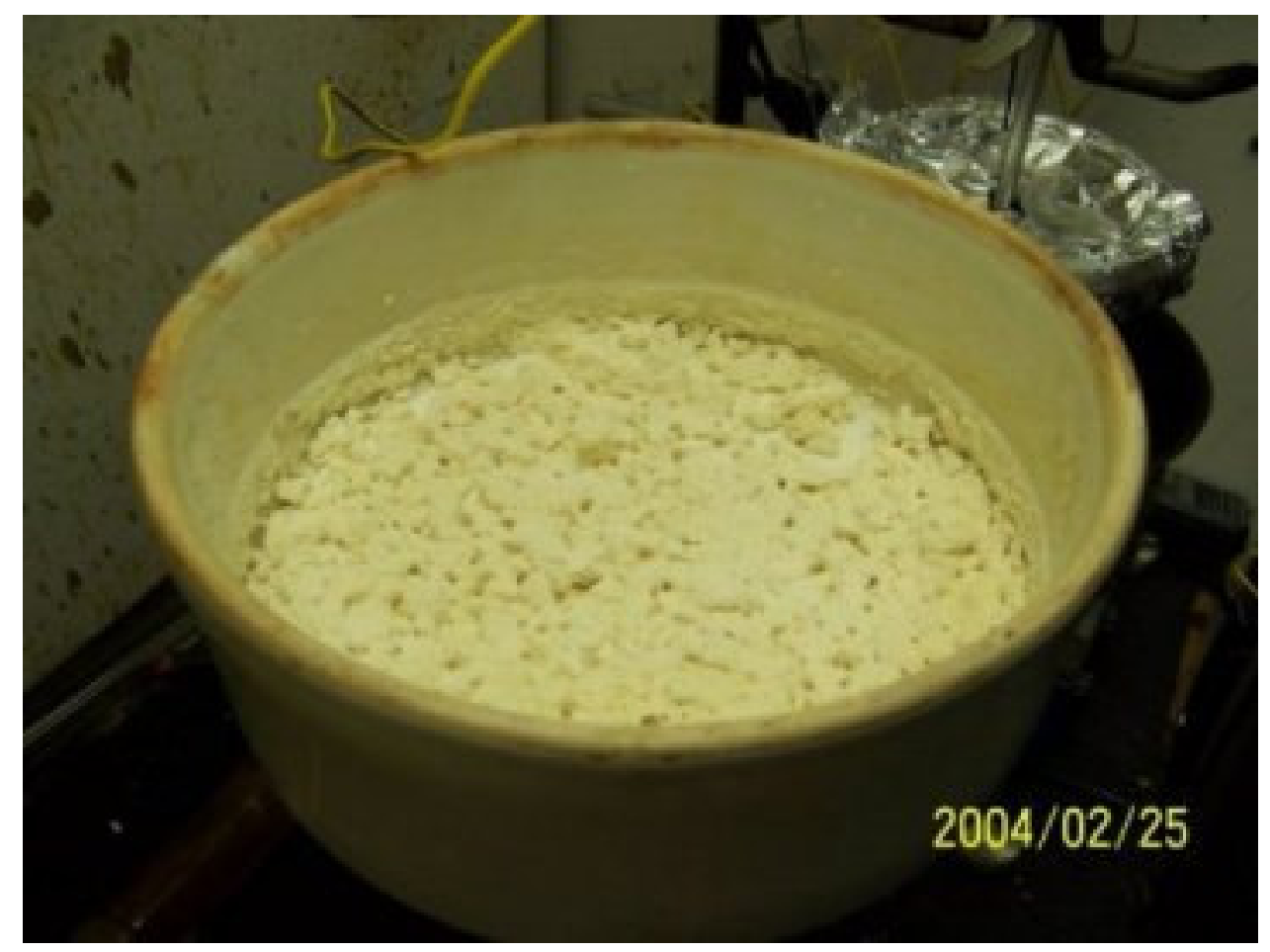

Figure 2.5 Microcapsule cakes drying in vacuum filter.

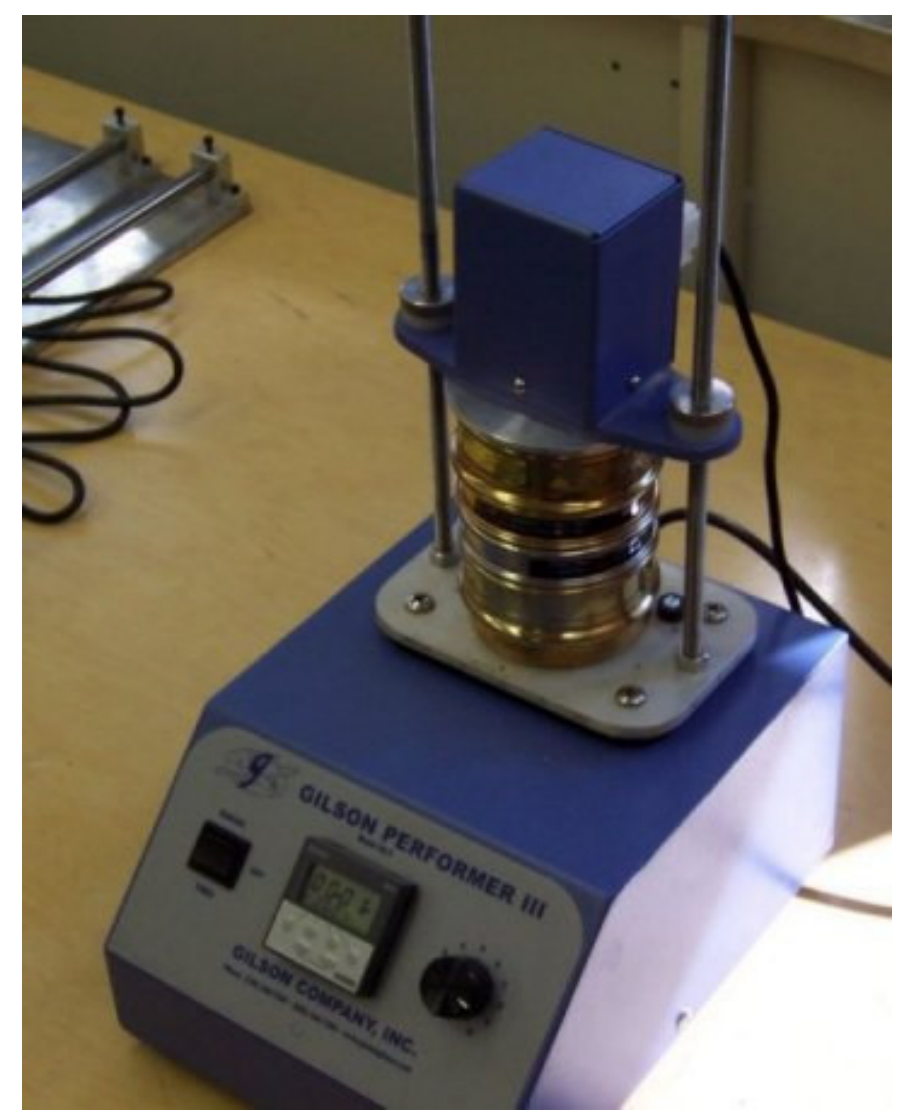

Figure 2.6 Gilson Performer III sieve shaker and sieve trays. 


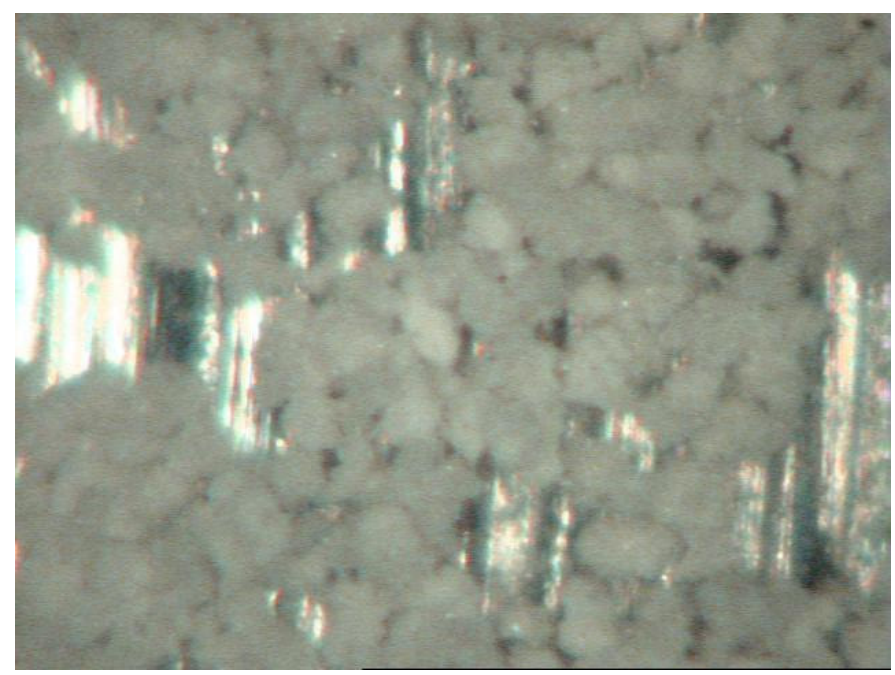

Figure 2.7 Urea-formaldehyde clumps

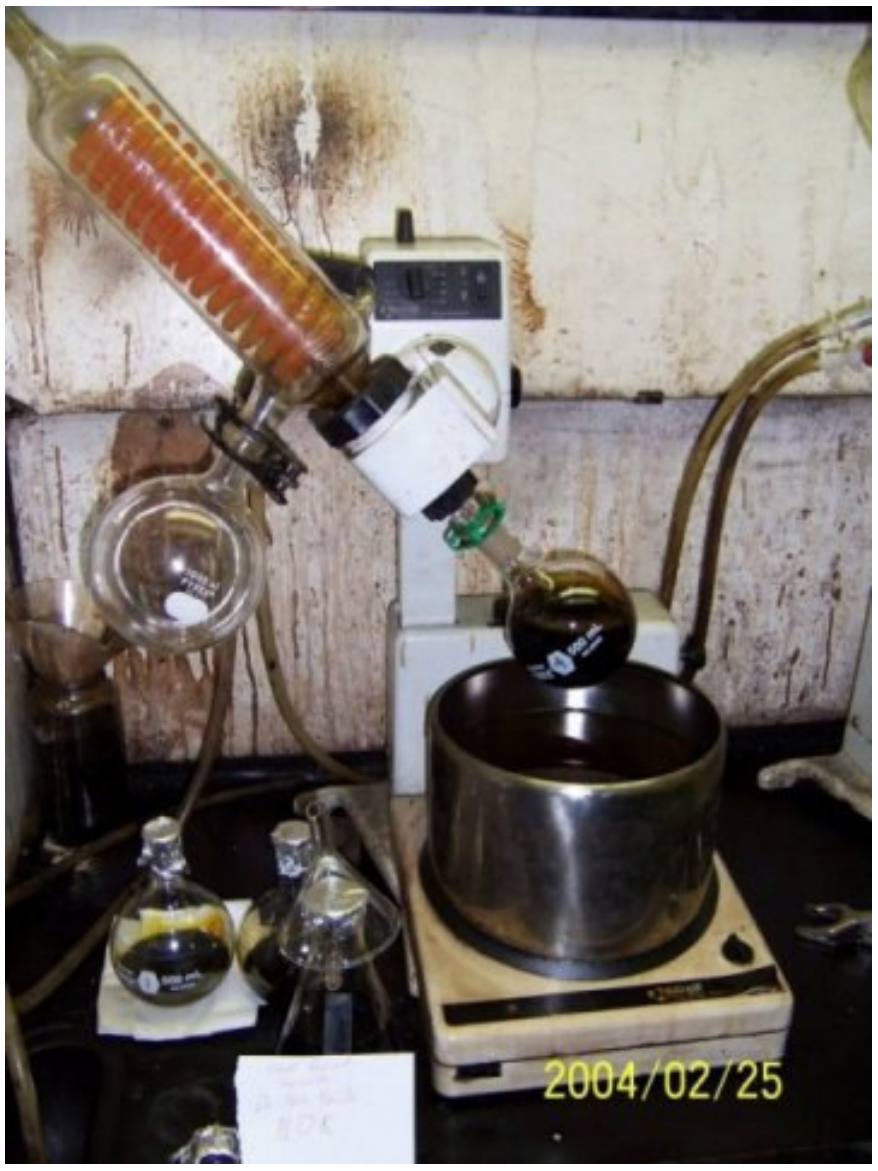

Figure 2.8 Rotavap distillation setup. 


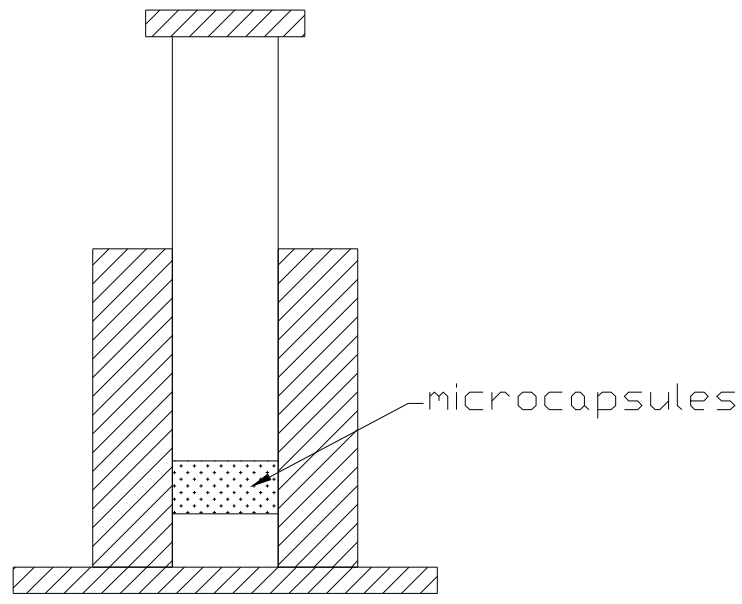

Figure 2.9 Instron testing machine

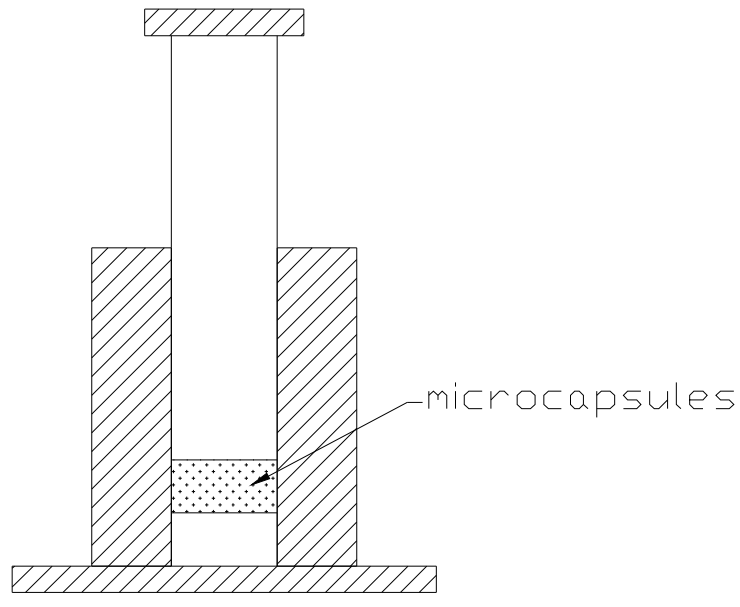

Figure 2.10 Compression test apparatus 


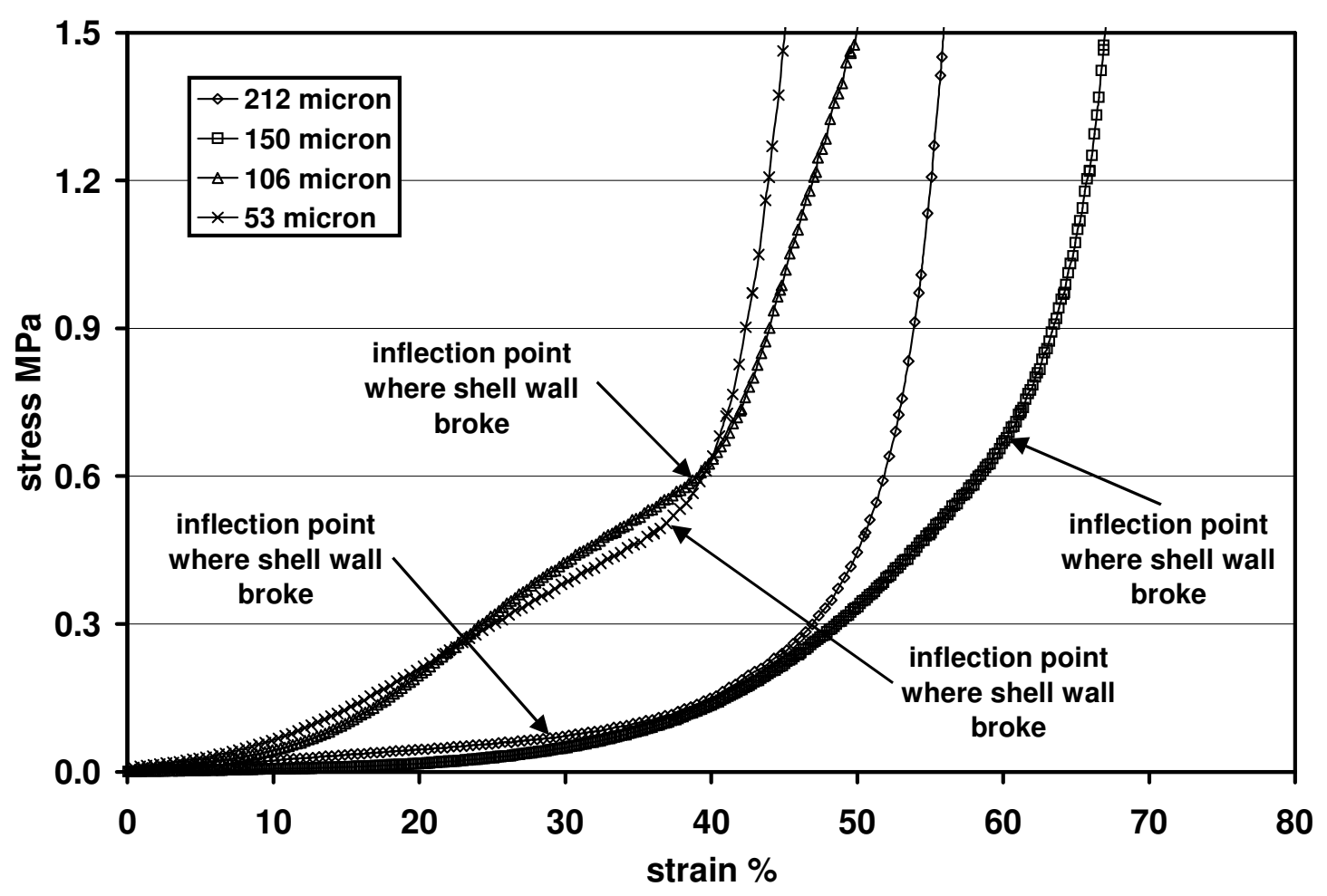

Figure 2.11 Stress vs. strain compression data form microcapsules

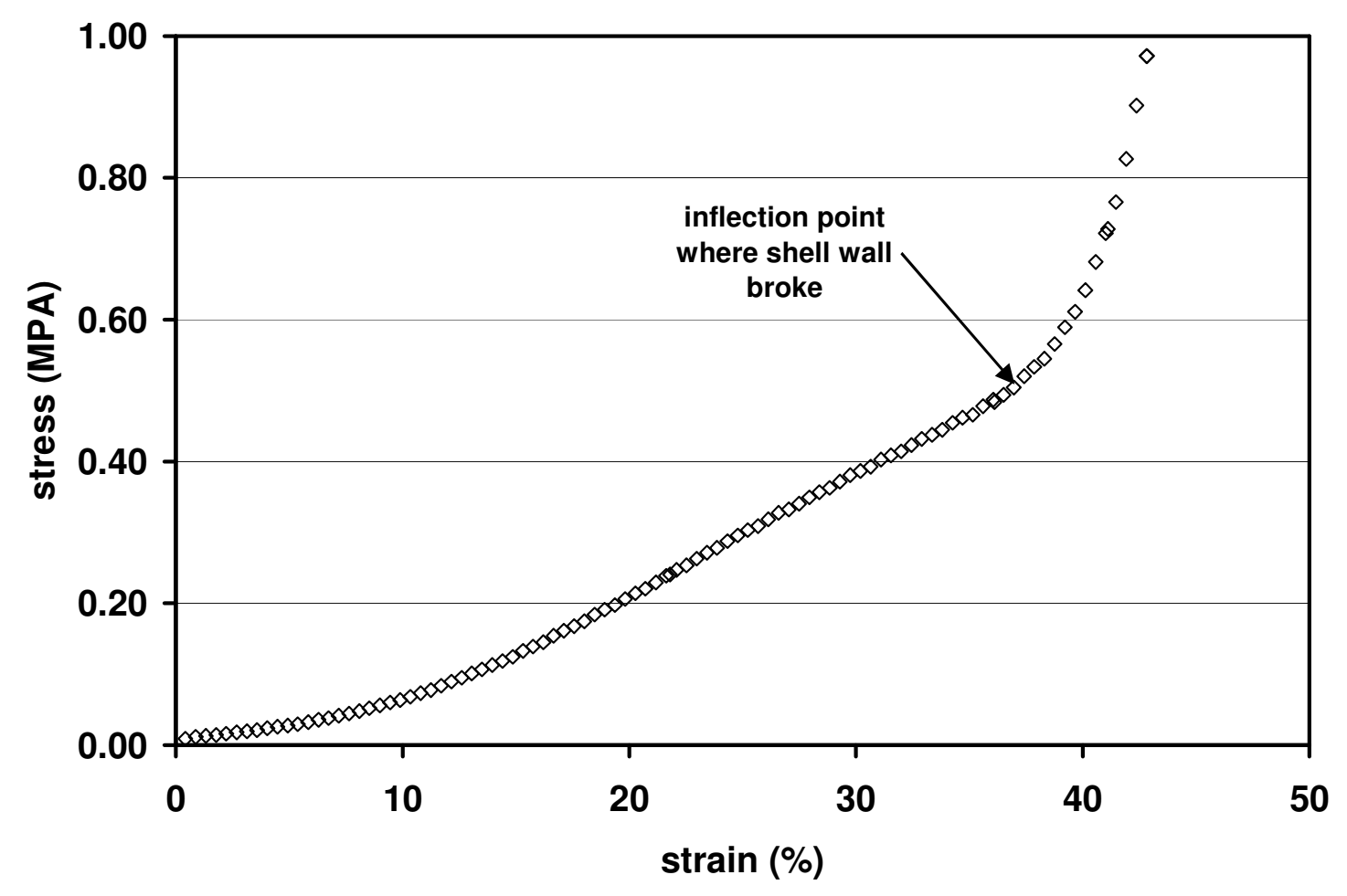

Figure 2.12 $53 \mu \mathrm{m}$ diameter stress strain curve 


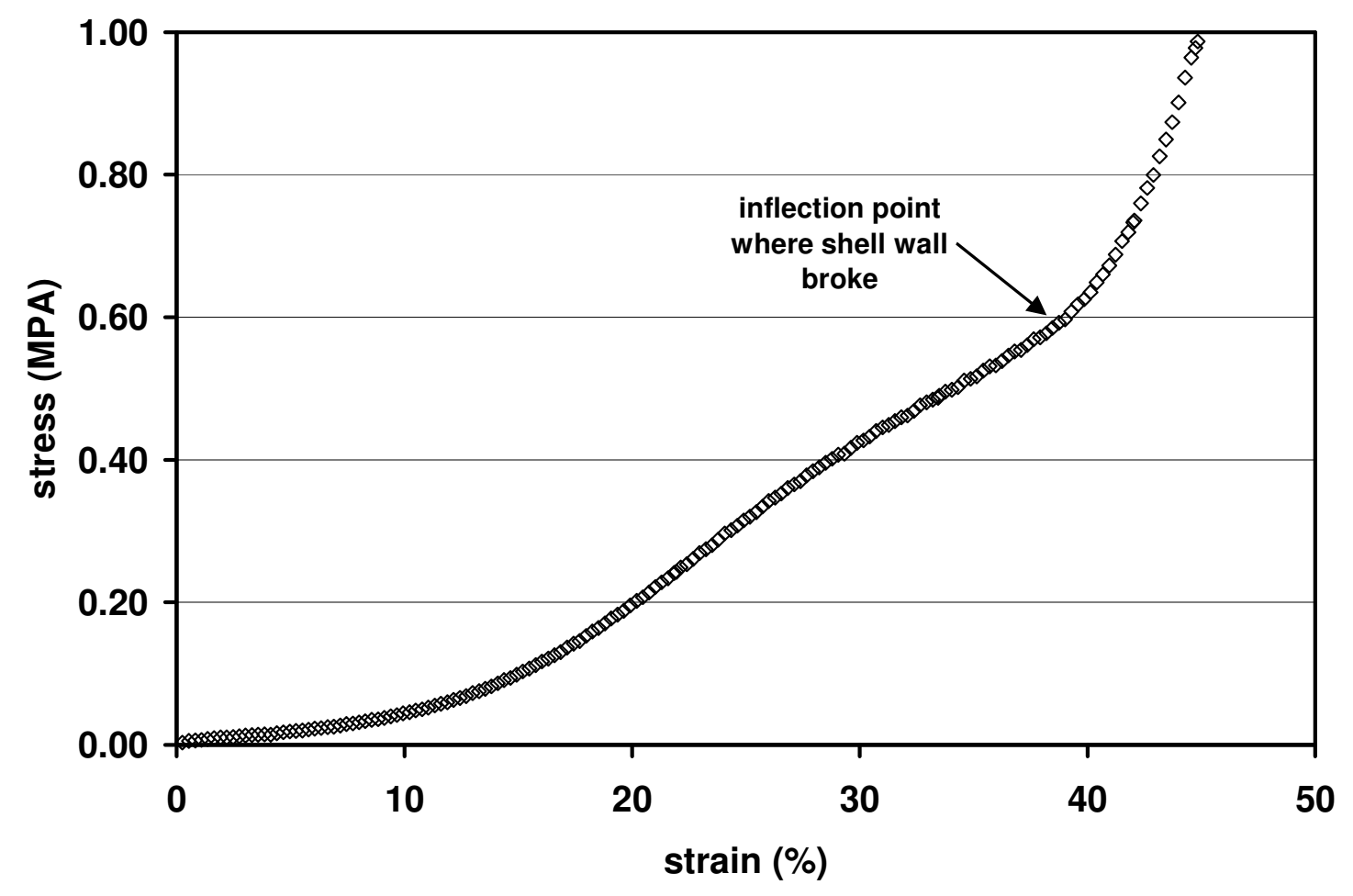

Figure 2.13 $106 \mu \mathrm{m}$ diameter stress strain curve

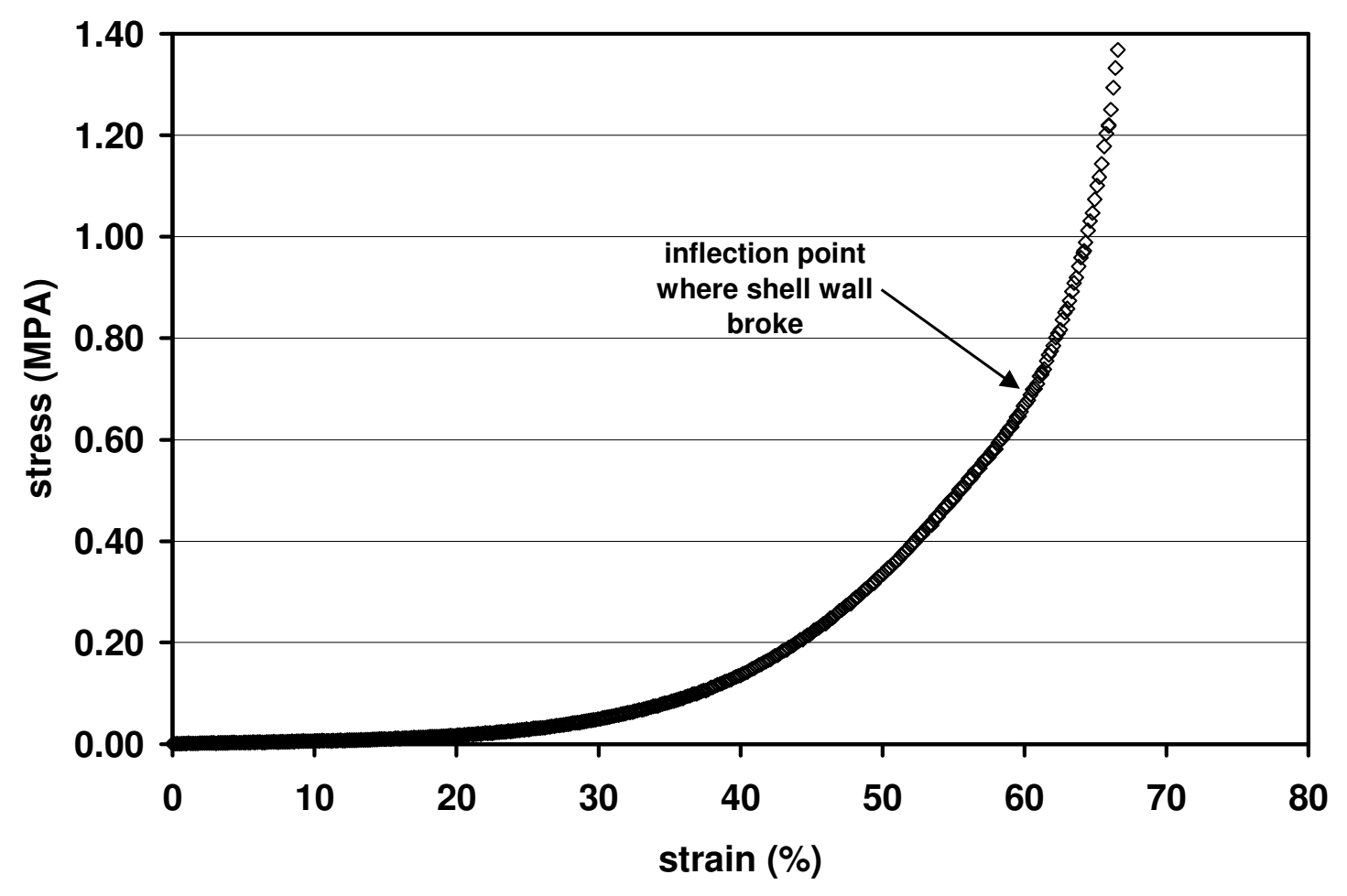

Figure $2.14150 \mu \mathrm{m}$ diameter stress strain curve 


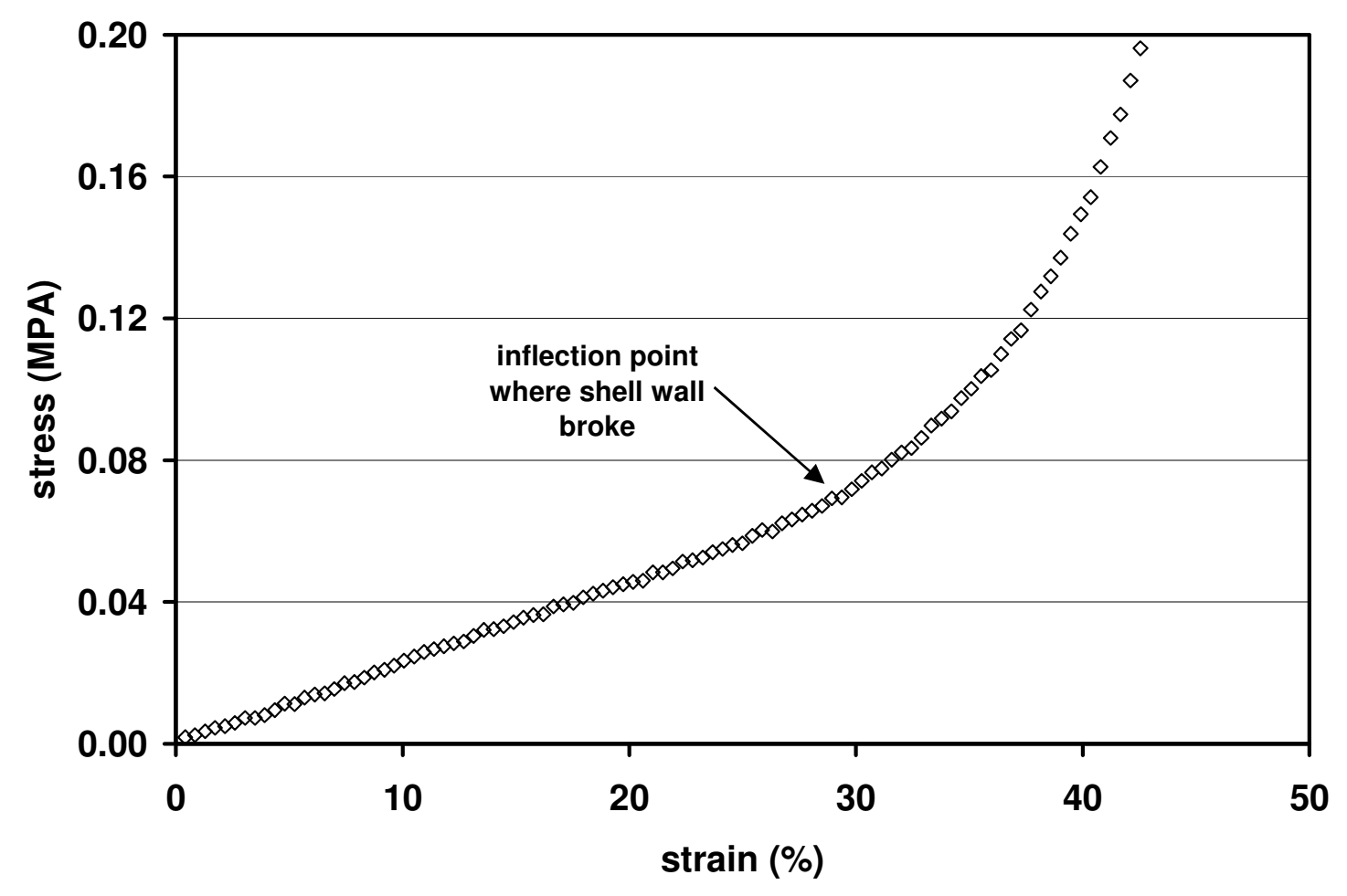

Figure 2.15 $212 \mu \mathrm{m}$ diameter stress strain curve

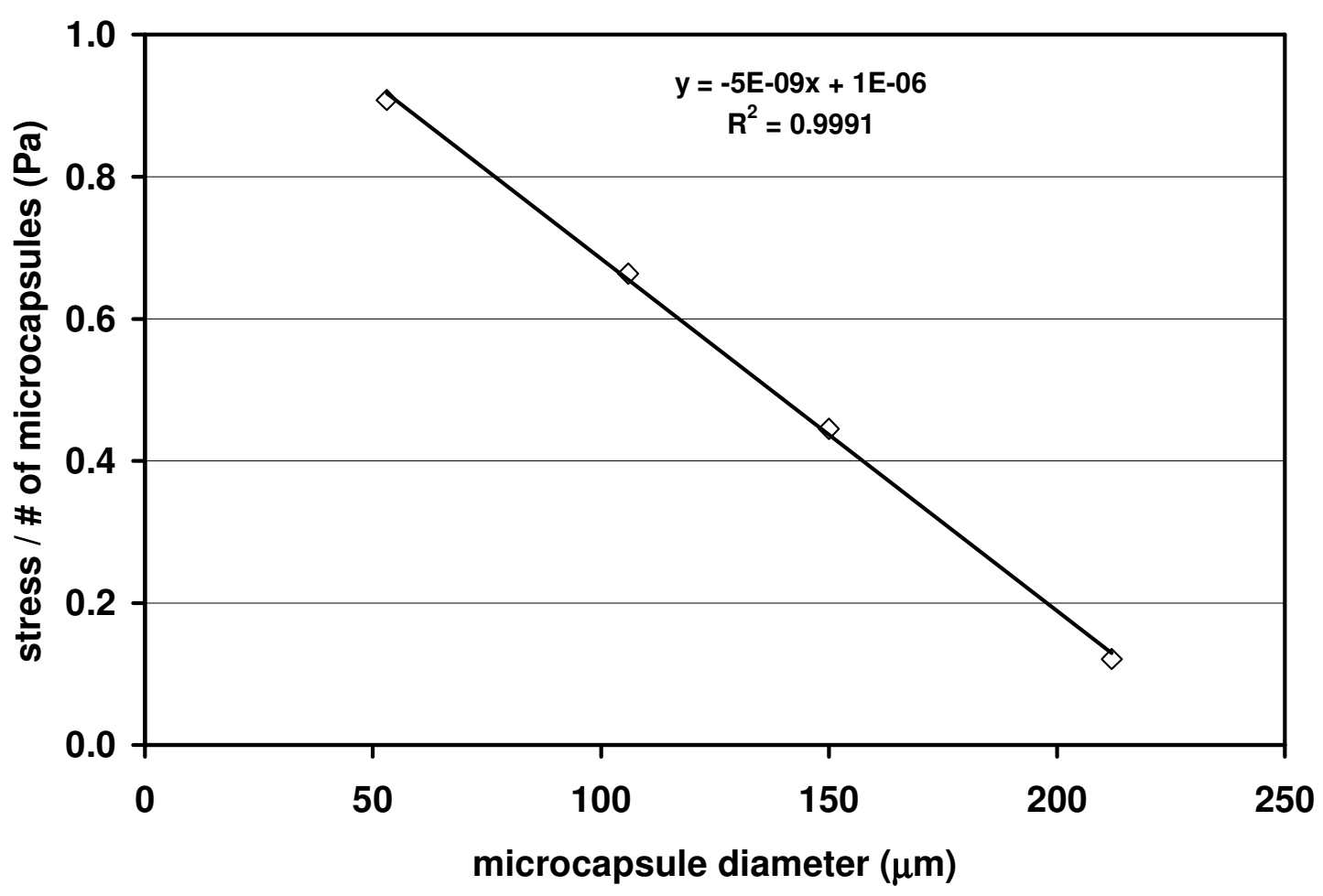

Figure 2.16 Microcapsules strength versus microcapsule size. 


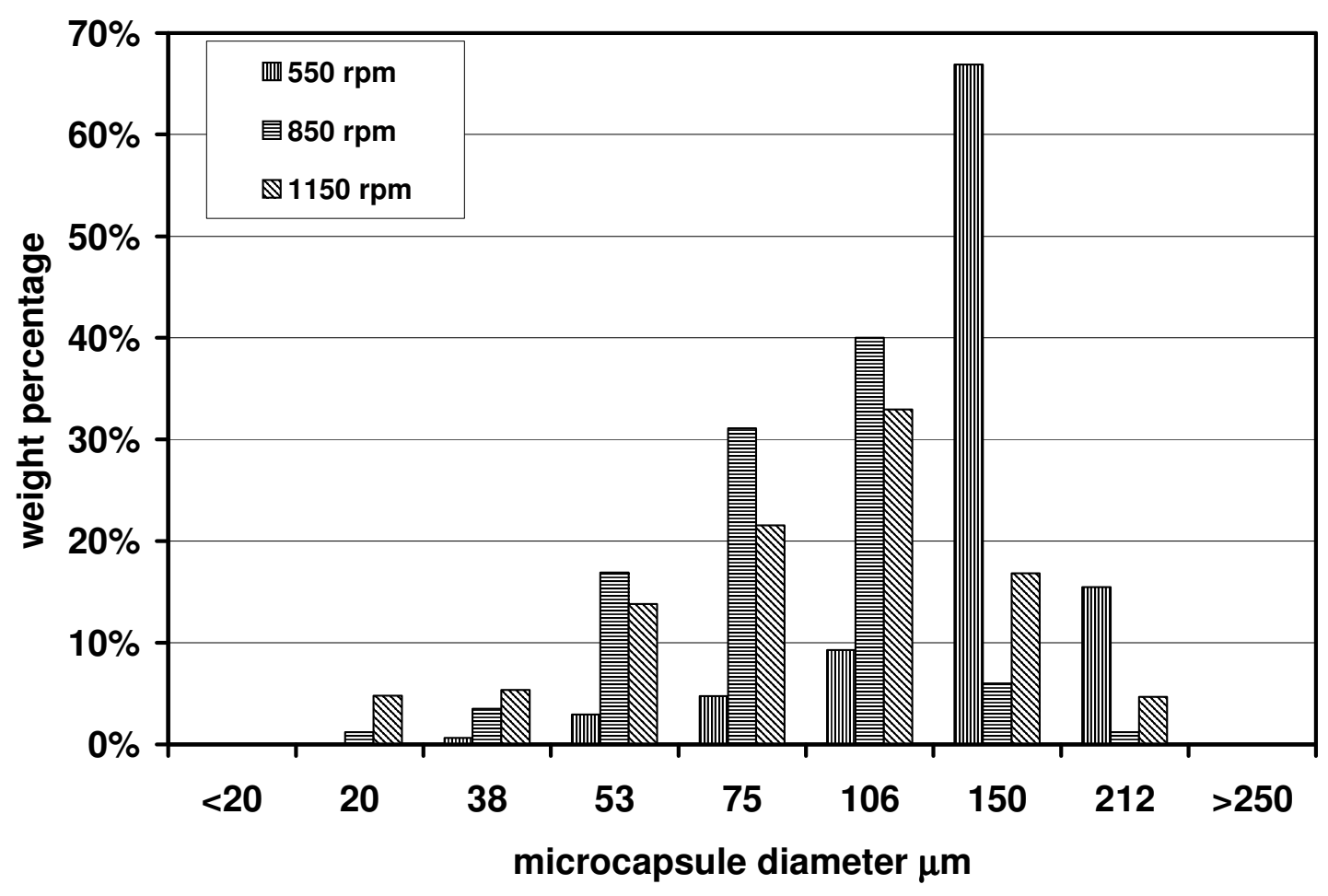

Figure 2.17 Size distribution of microcapsules at agitation rates of $550 \mathrm{rpm}, 850$ rpm, and $1150 \mathrm{rpm}$.

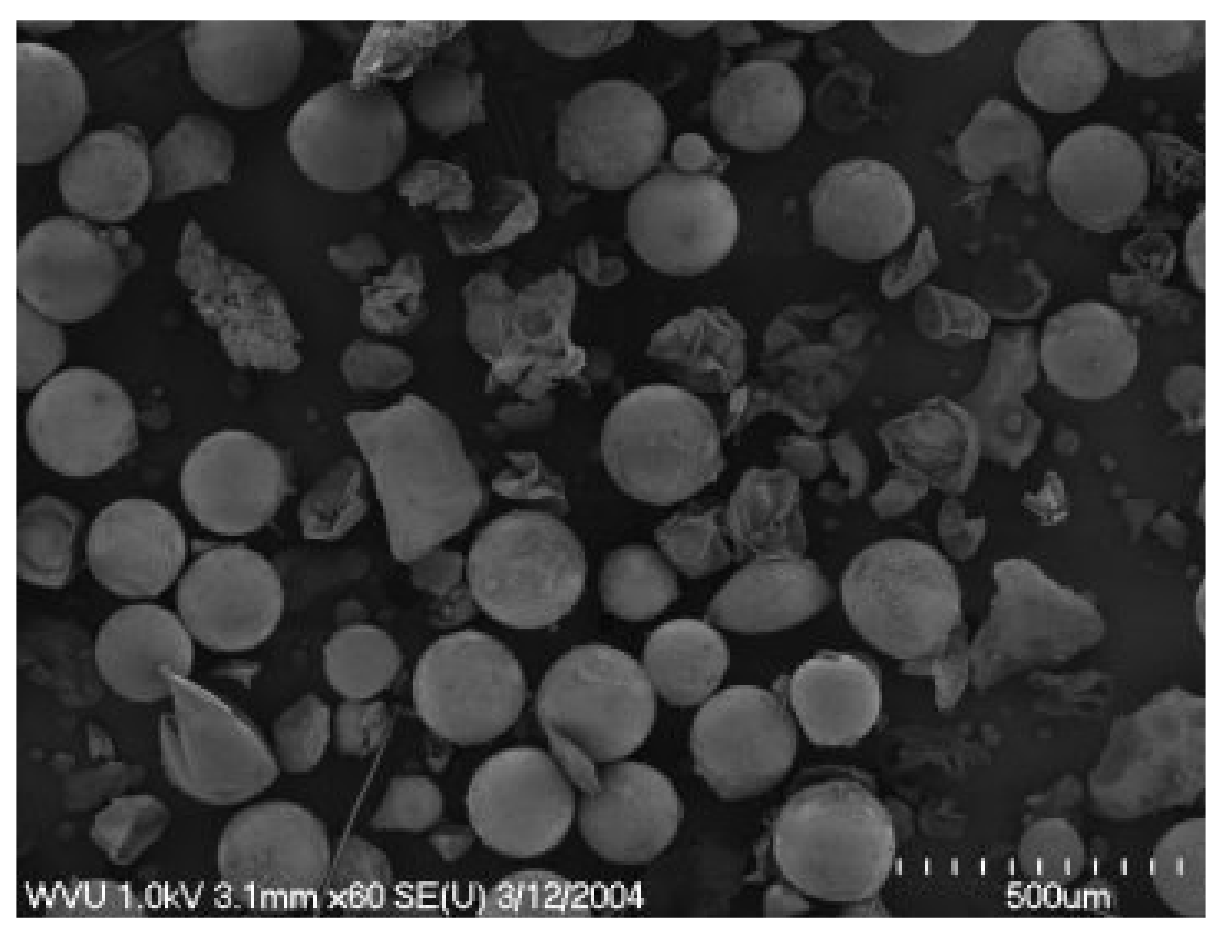

Figure 2.18 Microcapsules and urea-formaldehyde clumps at x60 magnification 


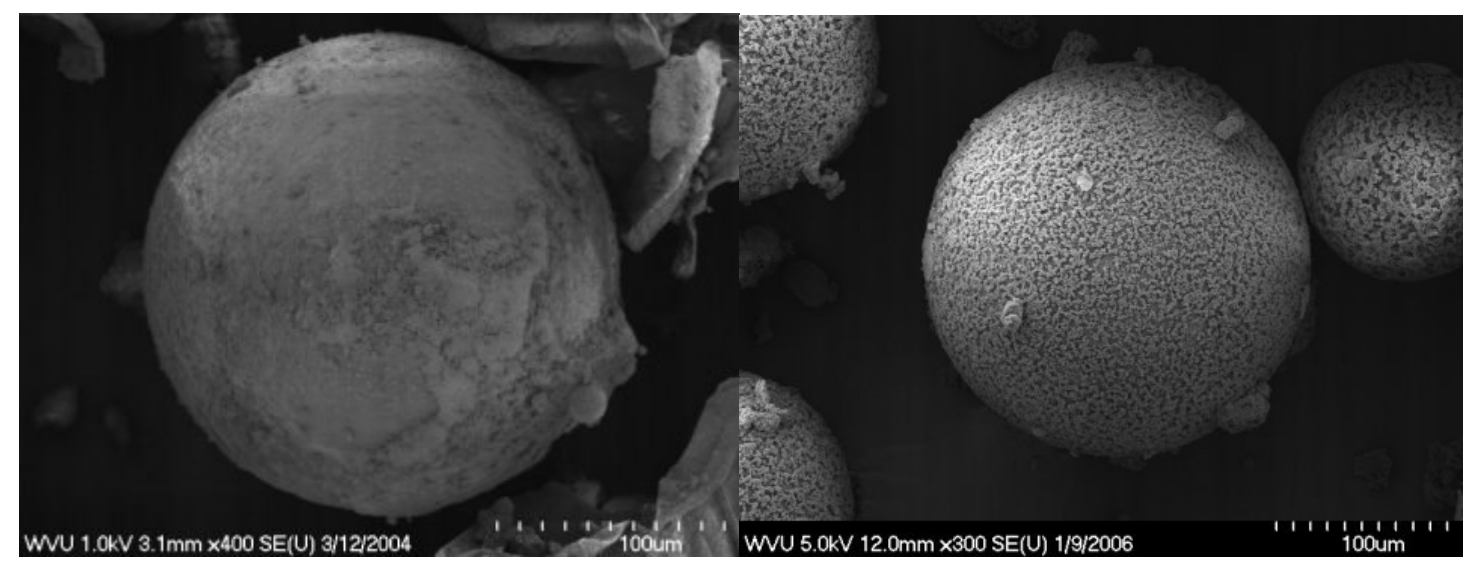

Figure 2.19 Microcapsules, approximately $200 \mu \mathrm{m}$ in diameter, x400 magnification and x300 magnification respectively.

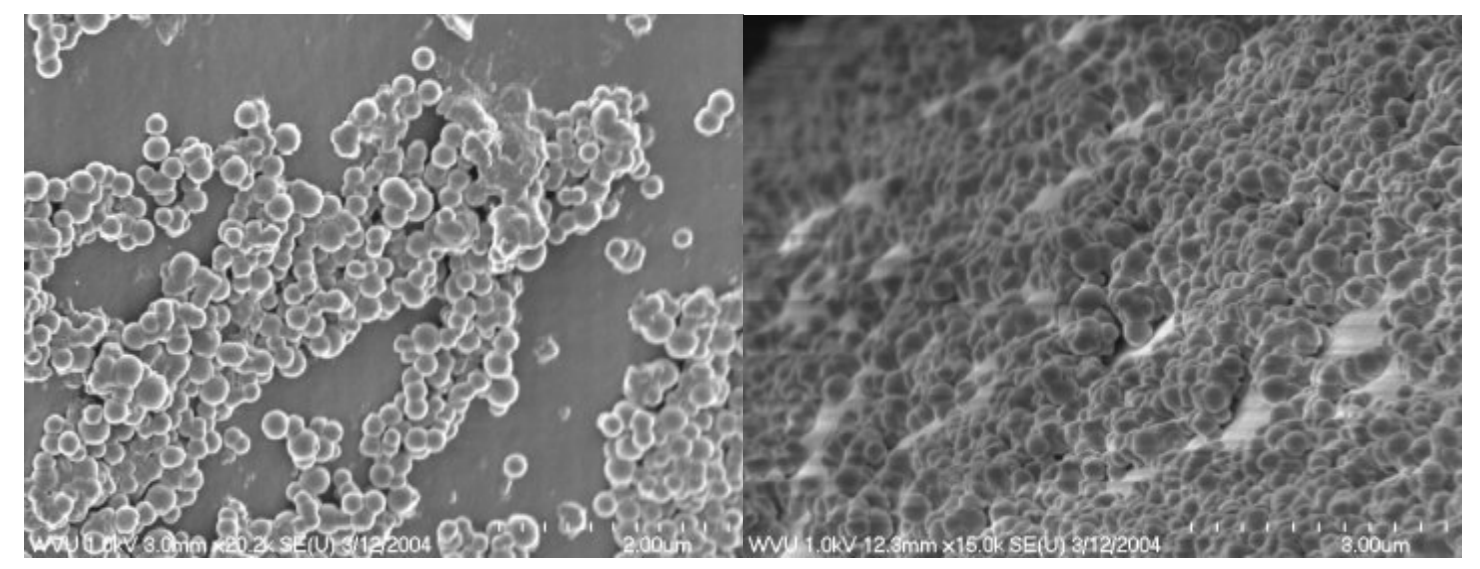

Figure 2.20 Microcapsule outer surface, notice the roughness of the outer surface, the shell wall is smooth, the leftover urea-formaldehyde clumps adhere to the surface making it rough, $x 20.2 \mathrm{k}$ and $\times 15.0 \mathrm{k}$ magnification, respectively 


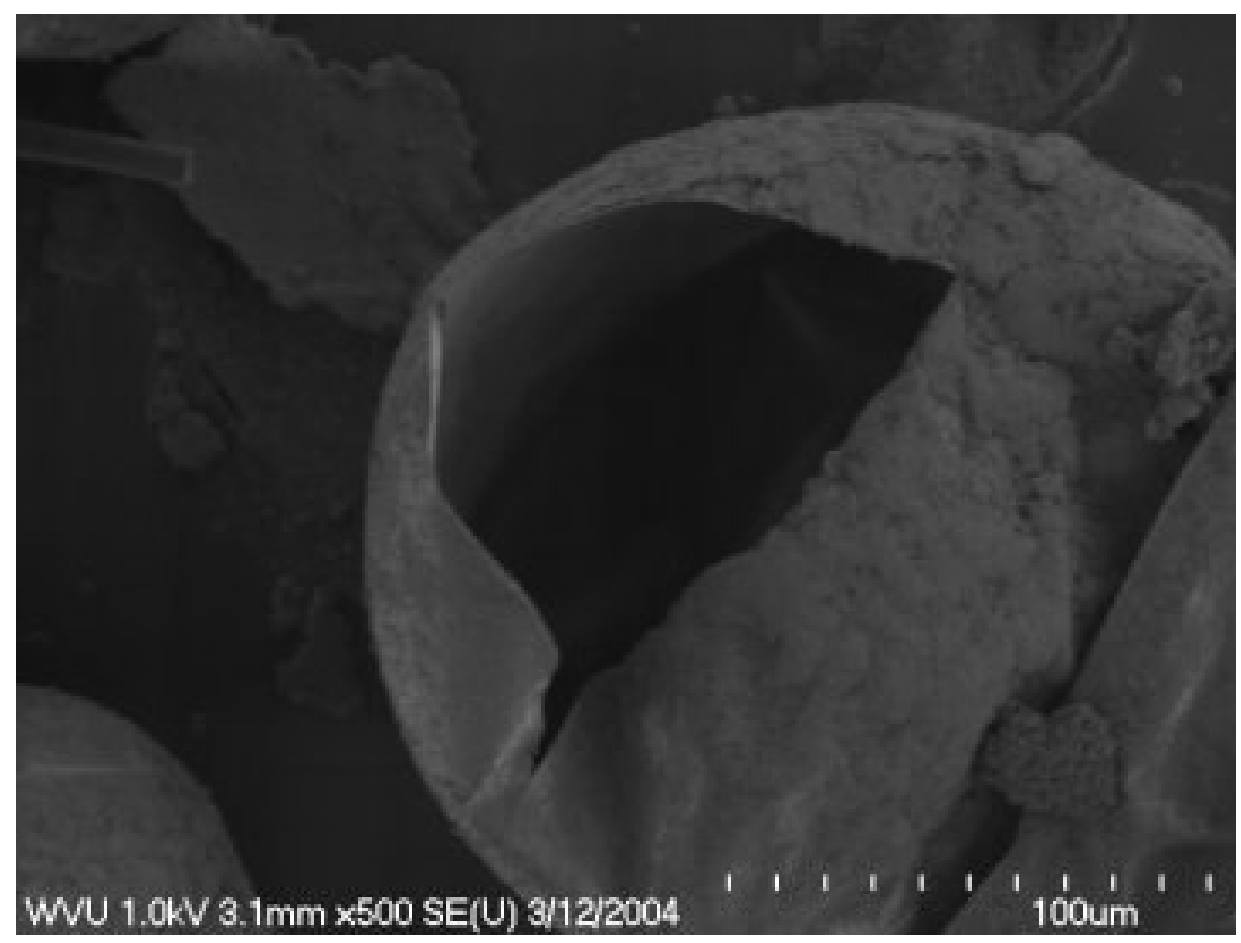

Figure 2.21 Broken microcapsule, notice the inner surface is smooth, compared to the rough outer surface $\mathbf{x 5 0 0}$

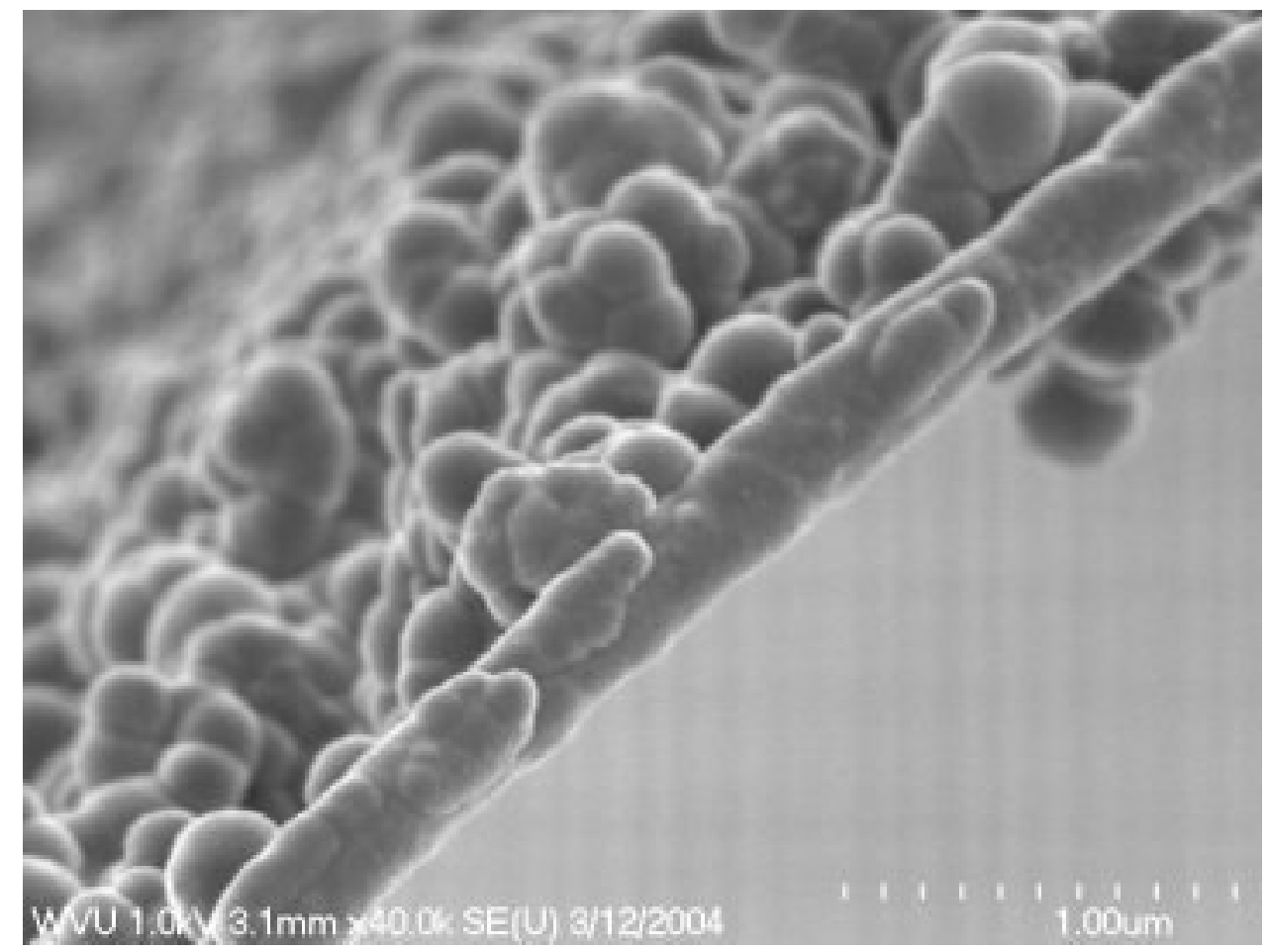

Figure 2.22 Broken microcapsule shell wall, approximately $0.30 \mu \mathrm{m}$ thick $x 40.0 \mathrm{k}$ magnification 


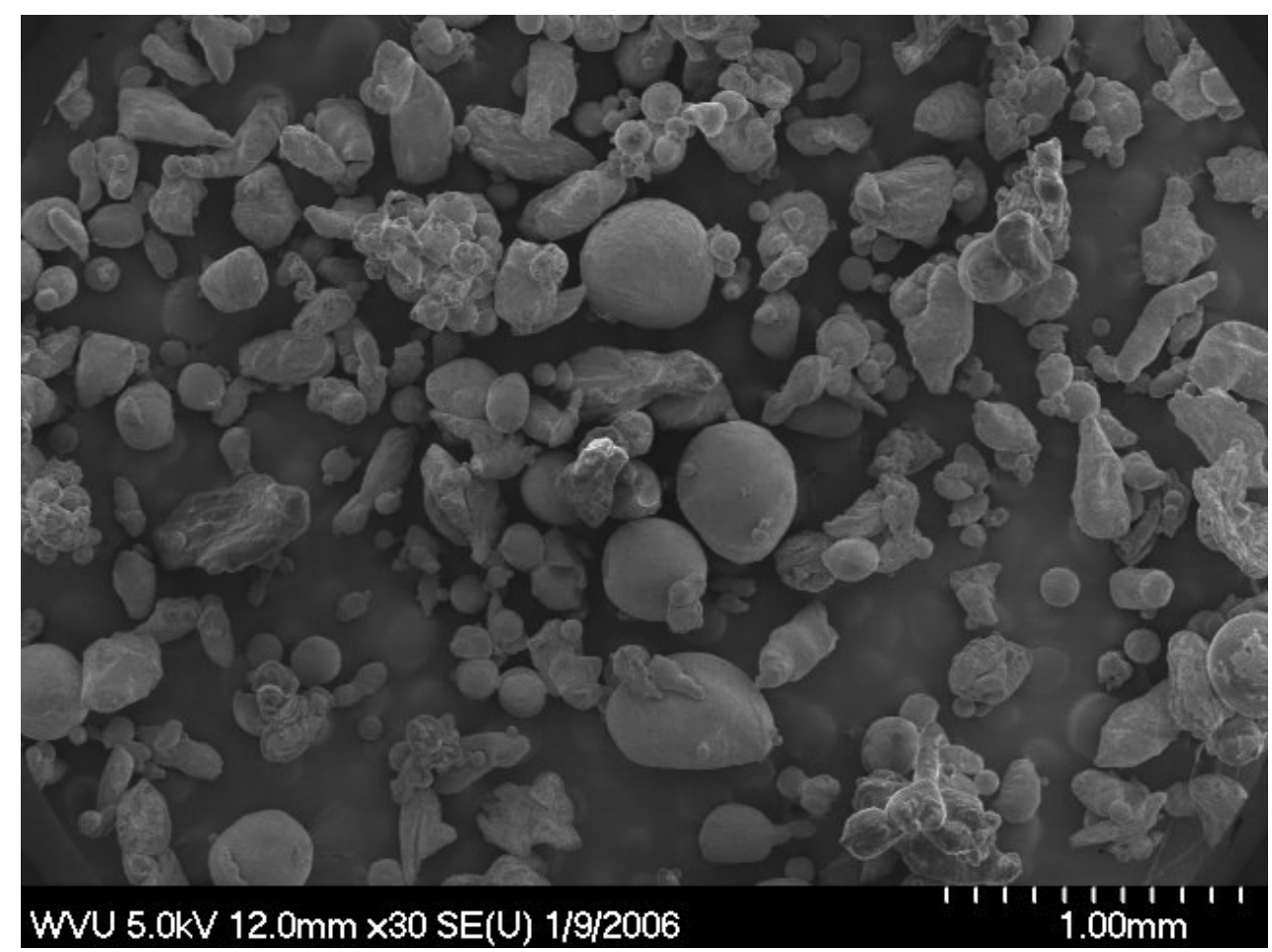

Figure 2.23 Grubbs' Ru catalyst encapsulated in wax at x30 magnification. 


\section{SPEICMEN FABRICATION AND TESTING}

The addition of the self-healing system, when compared to an equivalent material without the self-healing system, is thought to weaken the overall material properties. By performing the required tests for the CDHM model, for specimens containing the selfhealing system and specimens not containing the self-healing system, enough data will be available to show if there any degradation affects from the addition of the self-healing system. The required tests and testing methods for the CDHM model are outlined in this chapter.

\subsection{Specimen Fabrication}

Specimens are fabricated using hand lay-up and vacuum bagging techniques. Two flat aluminum plates are cleaned and then coated with at least two layers of Honey Wax (Specialty Products Company). Unidirectional $7 \mathrm{oz} / \mathrm{yd}^{2}$ fiberglass (Fibreglast) is then cut to the desired size, which is large enough to fabricate a plate that several specimens can be cut out from.

Once the dry fabricate is cut and the release agent has been applied, the resin, Epon 828, (Miller-Stephenson Chemical Company, Inc.) and hardener, Epicure 3223, (Miller-Stephenson Chemical Company, Inc.) are mixed according to manufacturing specifications (100:11 by weight). Each layer of dry fabric is then individually wetted with resin to avoid the brush bristles soaking up the microcapsules and catalyst. One layer of wet fabric is then placed on one of the aluminum plates. Based on UIUC's [23, $24,41,44]$ research a capsule concentration of $20 \%$ by weight and a catalyst concentration of $1.5 \%$ by weight was chosen. Microcapsules and wax encapsulated catalyst are measured based on their weight percentage, $20 \%$ and $10 \%$ (1.5\% catalyst), 
respectively. The total amount of microcapsules and catalyst needed for the lay-up is divided by $(n-1)$, where $n$ is the number of layers in the laminate. The divided amount of microcapsules and catalyst are then sprinkled on the wet layer of fabric. Each subsequent layer of fabric is then placed on top of the last and additional microcapsules and catalyst are sprinkled on the layer. This evenly spaces equal amounts of microcapsules and catalyst between each layer of fabric. Spacers, which are the thickness of the final specimen, are then placed on the steel plate around the wetted layers. The spacers are used to keep the aluminum plates from compressing the fibers, making the composite laminate thinner than desired. The other aluminum plate is then placed on top of the composite lay-up. The lay-up is then placed inside a vacuum bag and a vacuum of approximately $10 \mathrm{psi}$ is placed on the lay-up. C-clamps are then placed around the steel plates where each spacer is located (Figure 3.1). This helps to compress the aluminum plates together. Vacuum bagging removes air bubbles that can occur in the lay-up and the pressure from the vacuum compresses the two aluminum plates and the layers of fiberglass together.

The vacuum is kept on for approximately $12 \mathrm{hrs}$ at room temperature and the layup is allowed to cure for at least $48 \mathrm{hrs}$ before it is removed from between the aluminum plates. Specimens are then cut from the composite plate using one of several methods: a Dremel tool equipped with a Dremel carbide cutting/shaping wheel \#542 (Dremel), a wet tile cutting saw, and a milling machine using carbide coated end mills. This insures that the specimens are cut to the correct dimensions and that the edges are square and true. All specimens are cut to the testing standards outlined below. 


\subsection{Tensile Testing}

The fiber ultimate strain (stress) typically controls the axial tensile strength of a unidirectional composite lamina [35]. ASTM D 3039/D 3039M - 00 is used to determine the in-plane tensile properties of the specimens. The test takes a thin flat strip of the composite material with a constant rectangular cross section that is mounted in a mechanical testing machine and loaded in tension while recording the load. Several factors may influence the tensile properties such as: material, methods of material preparation and lay-up, specimen stacking sequence, specimen preparation, specimen conditioning, environment of testing, specimen alignment and gripping, speed of testing, time at temperature, void content, and volume percent reinforcement. In our case the volume percent of the reinforcement, microcapsule and catalyst, will influence the tensile properties. The following properties, in the test direction, are obtained from the D 3039/D test:

- Ultimate tensile strength

- Ultimate tensile strain

- Tensile chord modulus of elasticity

- Poisson's ratio

- Transition strain

All coupon measurements are taken using a micrometer as specified by the standard. Three measurements, width and thickness, are taken; one at each end of the specimen and one at the center of the specimen. These measurements are then averaged. The standard requires that at least five specimens per condition be tested. The testing machine is an MTS 810 (Figure 3.2), which is in conformance with Practices E 4 and 
satisfies the requirements outlined in the standard. The dimensions for specimens with a fiber orientation of $0^{\circ}$ unidirectional, longitudinal direction, are $15 \mathrm{~mm}$ wide, $250 \mathrm{~mm}$ long, and $1.0 \mathrm{~mm}$ thick. The corresponding tab dimensions are $56 \mathrm{~mm}$ in length, $1.5 \mathrm{~mm}$ thick, and a tab bevel angle of $90^{\circ}$. The dimensions for specimens with a fiber orientation of $90^{\circ}$ unidirectional, transverse direction are $25 \mathrm{~mm}$ wide, $175 \mathrm{~mm}$ long, and $2.0 \mathrm{~mm}$ thick. The corresponding tab dimensions are $25 \mathrm{~mm}$ in length, $1.5 \mathrm{~mm}$ thick, and a tab bevel angle of $90^{\circ}$. The material coordinate system is illustrated in Figure 3.3. An active gage length of $6 \mathrm{~mm}(0.25 \mathrm{in}$.) was used. During testing a constant head displacement rate of $2 \mathrm{~mm} / \mathrm{min}$ (0.05 in./min) was used. Vishay Micro-Measurements Tensile Gages CEA-06-250UN-350 were used based on recommendations from the manufacture.

The ultimate tensile strength, $F_{1 t}$ in the fiber direction is calculated using the following equation

$$
F_{1 t}=\frac{P^{\max }}{A}
$$

where $\mathrm{A}$ is the average cross-sectional area and $\mathrm{P}^{\max }$ is the maximum load before failure.

The tensile stress at each point, $\sigma_{i}$, is calculated using the following equation

$$
\sigma_{i}=\frac{P_{i}}{A}
$$

where $P_{i}$ is the load at the $i^{\text {th }}$ data point.

The tensile chord modulus of elasticity, $E_{1}$, is calculated from the stress-strain data using the following equation

$$
E_{1}=\frac{\Delta \sigma}{\Delta \varepsilon}
$$


where $\Delta \varepsilon$ is the difference between two strain points measured at approximately 1000 and $3000 \mu \varepsilon$ and $\Delta \sigma$ is the difference in applied tensile stress between the two strain points.

Poisson's ratio is calculated using the following equation

$$
v_{12}=v_{13}=-\frac{\Delta \varepsilon_{t}}{\Delta \varepsilon_{l}}
$$

where $\Delta \varepsilon_{t}$ is the difference in lateral strain between two longitudinal strain points measured at approximately 1000 and $3000 \mu \varepsilon$ and $\Delta \varepsilon_{l}$ is the difference between the two longitudinal strain points.

The results from the tensile testing of specimens containing the self-healing system are found in Table 3.1 and Table 3.2 is a list of the material properties of specimens not containing the self-healing system. Graphs of the data for the above mentioned material properties are found in Figure 3.4, Figure 3.5, Figure 3.6, and Figure 3.7.

\subsection{Compression Testing}

Based on the work by Wen [80] the SACMA SRM 1R-94 test method is used to determine the compressive properties of oriented fiber-resin composites. Specimens fabricated as shown in Figure 3.8 are mounted in the specially designed fixture shown in Figure 3.9. The fixture is then placed in an Instron testing machine (Figure 3.10). By loading the specimen to failure the ultimate compressive strength is obtained. Loadstrain curves are used to obtain the modulus of the material in a separate test. The following properties, in the test direction, are obtained from the SACMA SRM 1R-94 test:

- Ultimate compressive strength 
- Ultimate compressive strain

- Compressive modulus of elasticity

According to the SACMA standard at least five specimens shall be tested per condition. The specimens are mounted in the compression fixture and the support plates are closed by tightening the bolts to $0.7-1.0$ Joules. The dimensions of the specimen are $12.7 \mathrm{~mm}$ wide, $80.772 \mathrm{~mm}$ long, and $1.016 \mathrm{~mm}$ thick. The tabs on the specimens are $12.7 \mathrm{~mm}$ wide, $38.1 \mathrm{~mm}$ long, and $1.5875 \mathrm{~mm}$ thick.

In order to determine the ultimate strength of the material, specimens are loaded at $1 \mathrm{~mm} / \mathrm{min}$ until failure and the maximum load carried by the specimen is recorded. The compressive strength of the specimen is calculated from the following equation

$$
\sigma_{u l t}=\frac{P}{b d}
$$

where $\mathrm{P}$ is the maximum load, $\mathrm{b}$ is the average width, and $\mathrm{d}$ is the average thickness.

The results from the compression testing of specimens containing the self-healing system are found in Table 3.1 and Table 3.2 is a list of the material properties of specimens not containing the self-healing system. Graphs of the data for the above mentioned material properties are found in Figure 3.11, Figure 3.12, Figure 3.13, and Figure 3.14.

\subsection{Shear Testing}

Shear strength and modulus are measured following the D5379 "Iosipescu" method. Vishay Micro-Measurements Shear Gages N2P-08-C032A-500 were used since they average the shear strain over the entire region between the notches of the specimen [Wen]. This test uses a material coupon in the form of a rectangular flat strip with symmetrical centrally located v-notches as shown in Figure 3.15. The specimen is loaded 
by a MTS 810 (Figure 3.2) testing machine by a special fixture, shown in Figure 3.16. The following properties, in the test direction, can be obtained from the D 5379/D 5379M test:

- Shear stress/strain response

- Ultimate strength

- Ultimate strain

- Shear chord modulus of elasticity

- Transition strain

As mentioned earlier care must be taken during fabrication of the specimens to avoid material data scatter. According to the standard the specimen dimensions are 20.00 $\mathrm{mm}$ wide, $76.00 \mathrm{~mm}$ in length, and $2.00 \mathrm{~mm}$ thick. The $\mathrm{V}$ notches are cut into the specimen as shown in Figure 5.6 with the notch located $5.50 \mathrm{~mm}$ from the centerline of the specimen. The tabs are $2.5 \mathrm{~mm}$ thick and $32.0 \mathrm{~mm}$ in length. It has been shown that when testing in the 1-2 direction, the most accurate measurements of in-plane shear modulus for unidirectional materials result from $[0 / 90]_{n s}$ specimens $[10]$.

The ultimate shear strength is calculated from the following equation

$$
\tau_{u l t}=\frac{P}{A}
$$

where the area, A, is calculated as

$$
A=w_{n} \times t_{n}
$$

where $\mathrm{w}_{\mathrm{n}}$ is the width of the specimen measured at the notch and $t_{\mathrm{n}}$ is the thickness of the specimen measured at the notch. The shear stress at each data point is calculated as

$$
\tau_{i}=\frac{P_{i}}{A}
$$


and the shear strain at each data point is measured from directly from the strain gage.

The shear chord modulus of elasticity is calculated using Equation (3.9) over a $4000 \pm 200-\mu \varepsilon$ strain range, starting with the lower strain point in the range of 1500 to $2500 \mu \varepsilon$.

$$
G^{\text {chord }}=\frac{\Delta \tau}{\Delta \gamma}
$$

where $\Delta \tau$ is the difference in applied shear stress between the two strain points and $\Delta \gamma$ is the difference between the two strain points. For the specimens in this study the shear chord modulus of elasticity was calculated over the range of 1500 to $5500 \mu \varepsilon$.

The results from the tensile testing of specimens containing the self-healing system are found in Table 3.1 and Table 3.2 is a list of the material properties of specimens not containing the self-healing system. Graphs of the data for the above mentioned material properties are found in Figure 3.17 and Figure 3.18.

\subsection{Self-Healing Effects}

Along with studying the effects that the addition of the self-healing system has on the overall material properties, the amount of stiffness that the self-healing system can recover was also studied. Shear testing is used to study the effects of the self-healing system due to its high nonlinear nature. The first step was to look at how a composite material, without the self-healing system, in shear, damages. Figure 3.19 is a specimen, not containing the self-healing system, which was loaded in cyclic shear loading. Figure 3.19 displays the nonlinear loading and unloading associated with shear. The nonlinearity during unloading coincides with the literature [51, 52], but not with the damage models developed by Barbero et al. [15, 16, 17, 18, 19, 20]. 
Figure 3.20 is the cyclic loading from Figure 3.19 shifted to the origin. Upon looking at the data presented in Figure 3.20 one can notice that with each successive reloading the slope for the shear stress strain curve is less than the preceding curve. This is because the material has damaged. The decrease in slope is displayed in Figure 3.21 as a function of load number. One can notice that with each successive load the slope, $\mathrm{G}_{12}$, of the shear stress vs. shear strain curve decreases.

Shear specimens containing the self-healing system were then loaded to various strains, not allowed to heal, unloaded, and then reloaded. Figure 3.22 illustrates the damage in a specimen containing the self-healing system. The specimen was loaded in shear to a shear strain of $2.5 \%$, unloaded, not allowed to heal, and then reloaded. Just like the previous mentioned specimen the reloading slope is less then the original slope due to damage.

However, in Figure 3.23, Figure 3.24, and Figure 3.25 the specimen was loaded to $1.5 \%, 2.5 \%$, and $3.0 \%$ strain respectively, unloaded, allowed to heal for 48 hours, and then reloaded. Unlike the specimens that did not contain the self-healing system or those that were not allowed to heal, the reloading slopes are not less than the original slope, they are almost identical. This is due to the healing of the specimen. Additional specimens were tested in a similar manner with similar results.

Next, cyclic healing shear tests were preformed. Figure 3.26 is a typical cyclic healing shear test. Figure 3.27, Figure 3.29, and Figure 3.31 illustrate cyclic healing shear test data that has been shifted to the origin. The data is shifted to the origin to illustrate the effects of the healing. Unlike the specimen shown in Figure 3.19, the reloading curve of the specimens in Figure 3.27, Figure 3.29, and Figure 3.31 does not 
decrease. Some of the slopes in these reloading curves actually increase as illustrated in Figure 3.28, Figure 3.30, and Figure 3.32. Again here we see the effects of the healing system. The data is much different than that of specimens not able or not allowed to heal. After multiple healings, where the self-healing system begins to run out, we begin to see the slopes decrease. Chapters 4 and 6 discuss how this data is used to develop the Continuous Damage and Healing Mechanics Model.

\subsection{Testing Conclusions}

Table 3.3 is a comparison of the material properties of E-glass/Epoxy obtained from the above the mentioned tests and material properties not containing the self-healing system. As originally thought the self-healing system does degrade the overall material properties of the composite material. The self-healing system does recover some of the stiffness of the material. Figure 3.33 further illustrates how when the material is allowed heal the slope of the reloading does increase. More research varying the self-healing system concentrations needs to be preformed in order to understand the full effects of the addition of the self-healing system. 
Table 3.1 Material properties of specimens containing $20 \%$ wt. of microcapsules and $10 \%$ wt. of wax encapsulated catalyst.

\begin{tabular}{cc|c|c|c}
\hline \multicolumn{2}{c|}{ Material Property } & $\begin{array}{c}\text { Number of } \\
\text { Specimens }\end{array}$ & $\begin{array}{c}\text { Standard } \\
\text { Deviation }\end{array}$ & $\begin{array}{c}\text { Coefficient of } \\
\text { Variation (\%) }\end{array}$ \\
\hline $\mathrm{E}_{1}(\mathrm{MPa})$ & 30571.63 & 7 & 4185.55 & 13.69 \\
\hline $\mathrm{E}_{2}(\mathrm{MPa})$ & 8699.16 & 7 & 829.37 & 9.53 \\
\hline $\mathrm{E}_{3}(\mathrm{MPa})$ & 8699.16 & 7 & 829.37 & 9.53 \\
\hline$v_{12}$ & 0.251 & 6 & 0.035 & 14.11 \\
\hline$v_{13}$ & 0.251 & 6 & 0.035 & 14.11 \\
\hline$v_{23}$ & $\mathrm{x}$ & $\mathrm{x}$ & $\mathrm{x}$ & $\mathrm{x}$ \\
\hline $\mathrm{G}_{12}(\mathrm{MPa})$ & 2547.81 & 5 & 207.56 & 8.15 \\
\hline $\mathrm{G}_{13}(\mathrm{MPa})$ & 2547.81 & 5 & 207.56 & 8.15 \\
\hline $\mathrm{G}_{23}(\mathrm{MPa})$ & $\mathrm{x}$ & $\mathrm{x}$ & $\mathrm{x}$ & $\mathrm{x}$ \\
\hline $\mathrm{F}_{1 \mathrm{c}}(\mathrm{MPa})$ & 232.90 & 5 & 59.28 & 25.45 \\
\hline $\mathrm{F}_{1 \mathrm{t}}(\mathrm{MPa})$ & 397.36 & 7 & 66.12 & 16.64 \\
\hline $\mathrm{F}_{2 \mathrm{c}}(\mathrm{MPa})$ & 109.83 & 5 & 109.83 & 10.62 \\
\hline $\mathrm{F}_{2 \mathrm{t}}(\mathrm{MPa})$ & 45.16 & 7 & 10.60 & 23.48 \\
\hline $\mathrm{F}_{3 \mathrm{c}}(\mathrm{MPa})$ & 109.83 & 5 & 11.67 & 10.62 \\
\hline $\mathrm{F}_{3 \mathrm{t}}(\mathrm{MPa})$ & 45.16 & 7 & 10.60 & 23.48 \\
\hline $\mathrm{F}_{4}(\mathrm{MPa})$ & $\mathrm{x}$ & $\mathrm{x}$ & $\mathrm{x}$ & $\mathrm{x}$ \\
\hline $\mathrm{F}_{5}(\mathrm{MPa})$ & 38.37 & 4 & 2.19 & 5.70 \\
\hline $\mathrm{F}_{6}(\mathrm{MPa})$ & 38.37 & 4 & 2.19 & 5.70 \\
\hline & & & & \\
\hline
\end{tabular}


Table 3.2 Material properties of fiberglass epoxy not containing the self-healing system with a fiber volume fraction of $52 \%$.

\begin{tabular}{cc|c|c|c}
\hline \multicolumn{2}{c|}{ Material Property } & $\begin{array}{c}\text { Number of } \\
\text { Specimens }\end{array}$ & $\begin{array}{c}\text { Standard } \\
\text { Deviation }\end{array}$ & $\begin{array}{c}\text { Coefficient of } \\
\text { Variation (\%) }\end{array}$ \\
\hline $\mathrm{E}_{1}(\mathrm{MPa})$ & 34784 & 5 & 2185.89 & 6.28 \\
\hline $\mathrm{E}_{2}(\mathrm{MPa})$ & 13469 & 3 & 13468.51 & 4.36 \\
\hline $\mathrm{E}_{3}(\mathrm{MPa})$ & 13469 & 3 & 13468.51 & 4.36 \\
\hline $\mathrm{v}_{12}$ & 0.255 & 5 & 0.032 & 12.61 \\
\hline$v_{13}$ & 0.255 & 5 & 0.032 & 12.61 \\
\hline $\mathrm{v}_{23}$ & $\mathrm{x}$ & $\mathrm{x}$ & $\mathrm{x}$ & $\mathrm{x}$ \\
\hline $\mathrm{G}_{12}(\mathrm{MPa})$ & 3043 & 5 & 439.74 & 14.45 \\
\hline $\mathrm{G}_{13}(\mathrm{MPa})$ & 3043 & 5 & 439.74 & 14.45 \\
\hline $\mathrm{G}_{23}(\mathrm{MPa})$ & $\mathrm{x}$ & $\mathrm{x}$ & $\mathrm{x}$ & $\mathrm{x}$ \\
\hline $\mathrm{F}_{1 \mathrm{c}}(\mathrm{MPa})$ & 459.1 & 5 & 43.66 & 9.51 \\
\hline $\mathrm{F}_{1 \mathrm{t}}(\mathrm{MPa})$ & 592.3 & 5 & 29.32 & 4.95 \\
\hline $\mathrm{F}_{2 \mathrm{c}}(\mathrm{MPa})$ & 109.5 & 6 & 9.25 & 8.45 \\
\hline $\mathrm{F}_{2 \mathrm{t}}(\mathrm{MPa})$ & 68.86 & 3 & 9.17 & 13.32 \\
\hline $\mathrm{F}_{3 \mathrm{c}}(\mathrm{MPa})$ & 109.5 & 6 & 9.25 & 8.45 \\
\hline $\mathrm{F}_{3 \mathrm{t}}(\mathrm{MPa})$ & 68.86 & 3 & 9.17 & 13.32 \\
\hline $\mathrm{F}_{4}(\mathrm{MPa})$ & $\mathrm{x}$ & $\mathrm{x}$ & $\mathrm{x}$ & $\mathrm{x}$ \\
\hline $\mathrm{F}_{5}(\mathrm{MPa})$ & 49.87 & 5 & 3.39 & 6.79 \\
\hline $\mathrm{F}_{6}(\mathrm{MPa})$ & 49.87 & 5 & 3.39 & 6.79 \\
\hline
\end{tabular}


Table 3.3 Comparison of material properties of specimens containing the selfhealing system and specimens not containing the self-healing system.

\begin{tabular}{cccc}
\hline $\begin{array}{c}\text { Material } \\
\text { Property }\end{array}$ & $\begin{array}{c}\text { without self- } \\
\text { healing system }\end{array}$ & $\begin{array}{c}\text { with self- } \\
\text { healing system }\end{array}$ & $\begin{array}{c}\text { percent } \\
\text { difference }\end{array}$ \\
\hline $\mathrm{E}_{1}(\mathrm{MPa})$ & 34784 & 30572 & $-12 \%$ \\
\hline $\mathrm{E}_{2}(\mathrm{MPa})$ & 13469 & 8699 & $-35 \%$ \\
\hline $\mathrm{E}_{3}(\mathrm{MPa})$ & 13469 & 8699 & $-35 \%$ \\
\hline$v_{12}$ & 0.255 & 0.251 & $\mathrm{x}$ \\
\hline$v_{13}$ & 0.255 & 0.251 & $\mathrm{x}$ \\
\hline$v_{23}$ & $\mathrm{x}$ & $\mathrm{x}$ & $\mathrm{x}$ \\
\hline $\mathrm{G}_{12}(\mathrm{MPa})$ & 3043 & 2859 & $-6 \%$ \\
\hline $\mathrm{G}_{13}(\mathrm{MPa})$ & 3043 & 2859 & $-6 \%$ \\
\hline $\mathrm{G}_{23}(\mathrm{MPa})$ & $\mathrm{x}$ & $\mathrm{x}$ & $\mathrm{x}$ \\
\hline $\mathrm{F}_{1 \mathrm{c}}(\mathrm{MPa})$ & 459 & 221 & $-52 \%$ \\
\hline $\mathrm{F}_{1 \mathrm{t}}(\mathrm{MPa})$ & 592 & 397 & $-33 \%$ \\
\hline $\mathrm{F}_{2 \mathrm{c}}(\mathrm{MPa})$ & 110 & 110 & $0 \%$ \\
\hline $\mathrm{F}_{2 \mathrm{t}}(\mathrm{MPa})$ & 68.86 & 45.16 & $-34 \%$ \\
\hline $\mathrm{F}_{3 \mathrm{c}}(\mathrm{MPa})$ & 110 & 110 & $0 \%$ \\
\hline $\mathrm{F}_{3 \mathrm{t}}(\mathrm{MPa})$ & 68.86 & 45.16 & $-34 \%$ \\
\hline $\mathrm{F}_{4}(\mathrm{MPa})$ & $\mathrm{x}$ & 36.70 & $-26 \%$ \\
\hline $\mathrm{F}_{5}(\mathrm{MPa})$ & 49.87 & & $-26 \%$ \\
\hline $\mathrm{F}_{6}(\mathrm{MPa})$ & 49.87 & 36.70 & \\
\hline
\end{tabular}




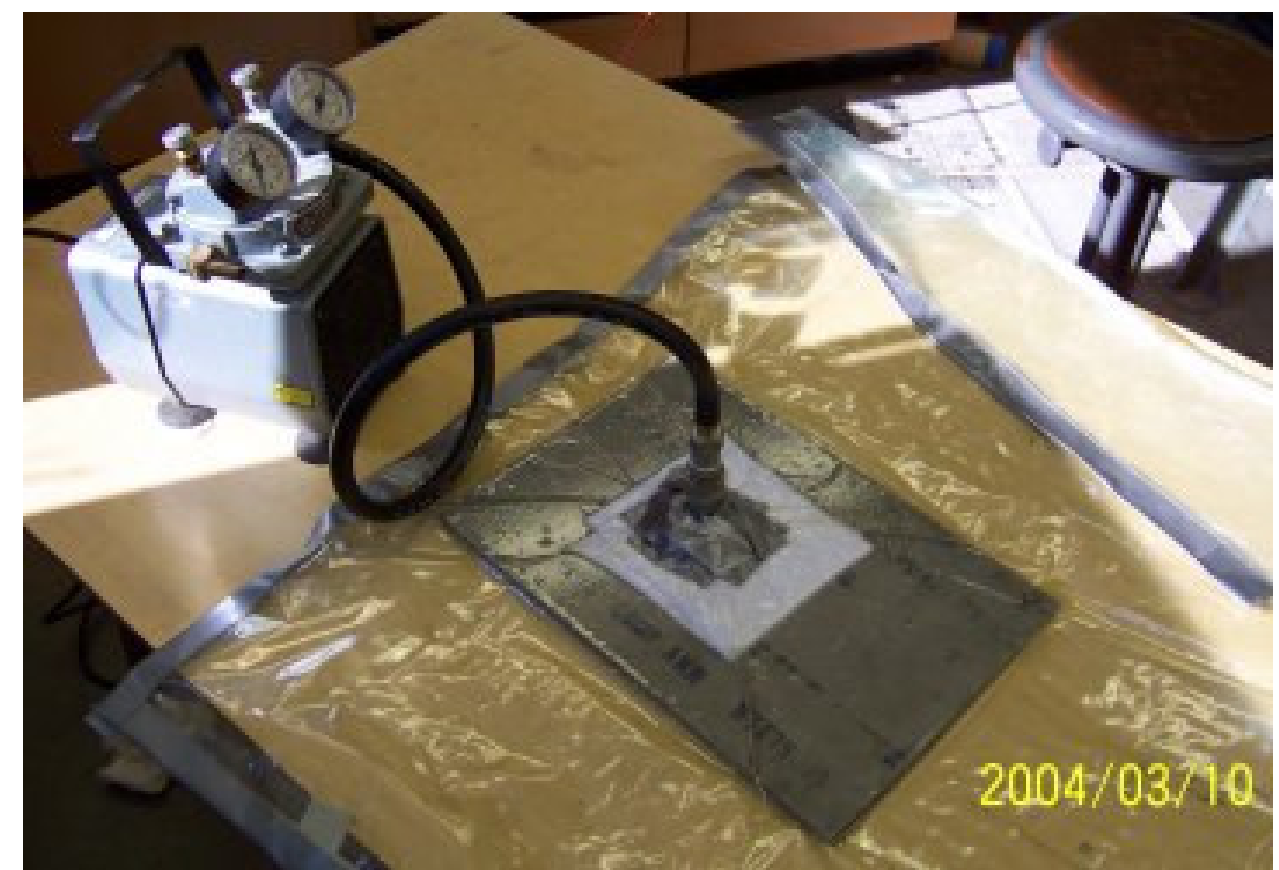

Figure 3.1 Hand lay-up and vacuum bagging of samples.

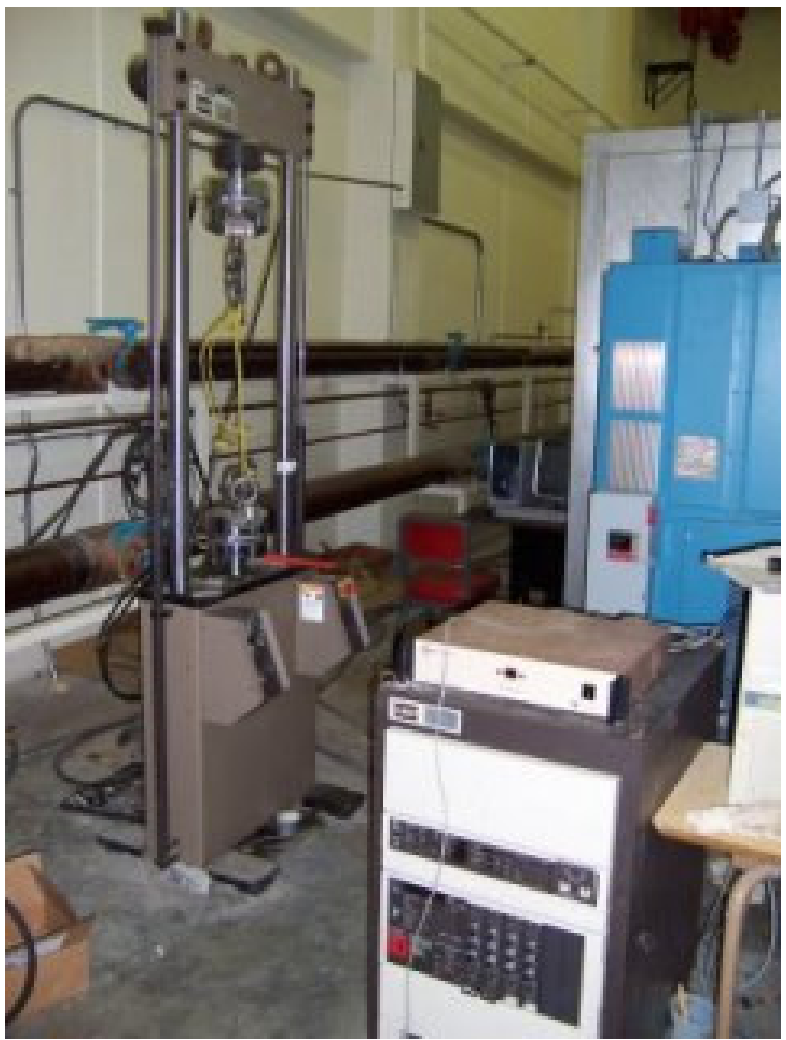

Figure 3.2 MTS 810 testing machine. 


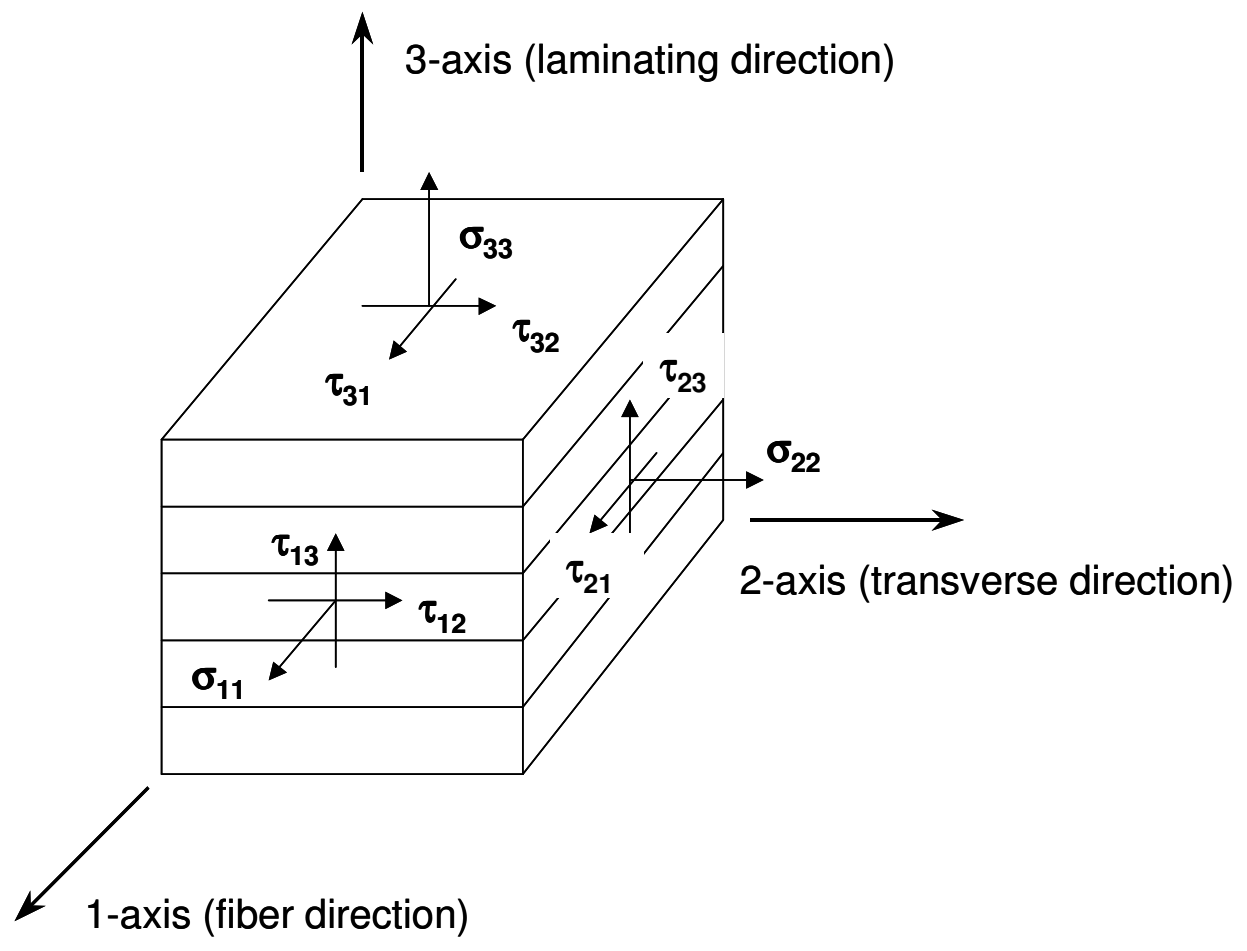

Figure 3.3 Material coordinate system.

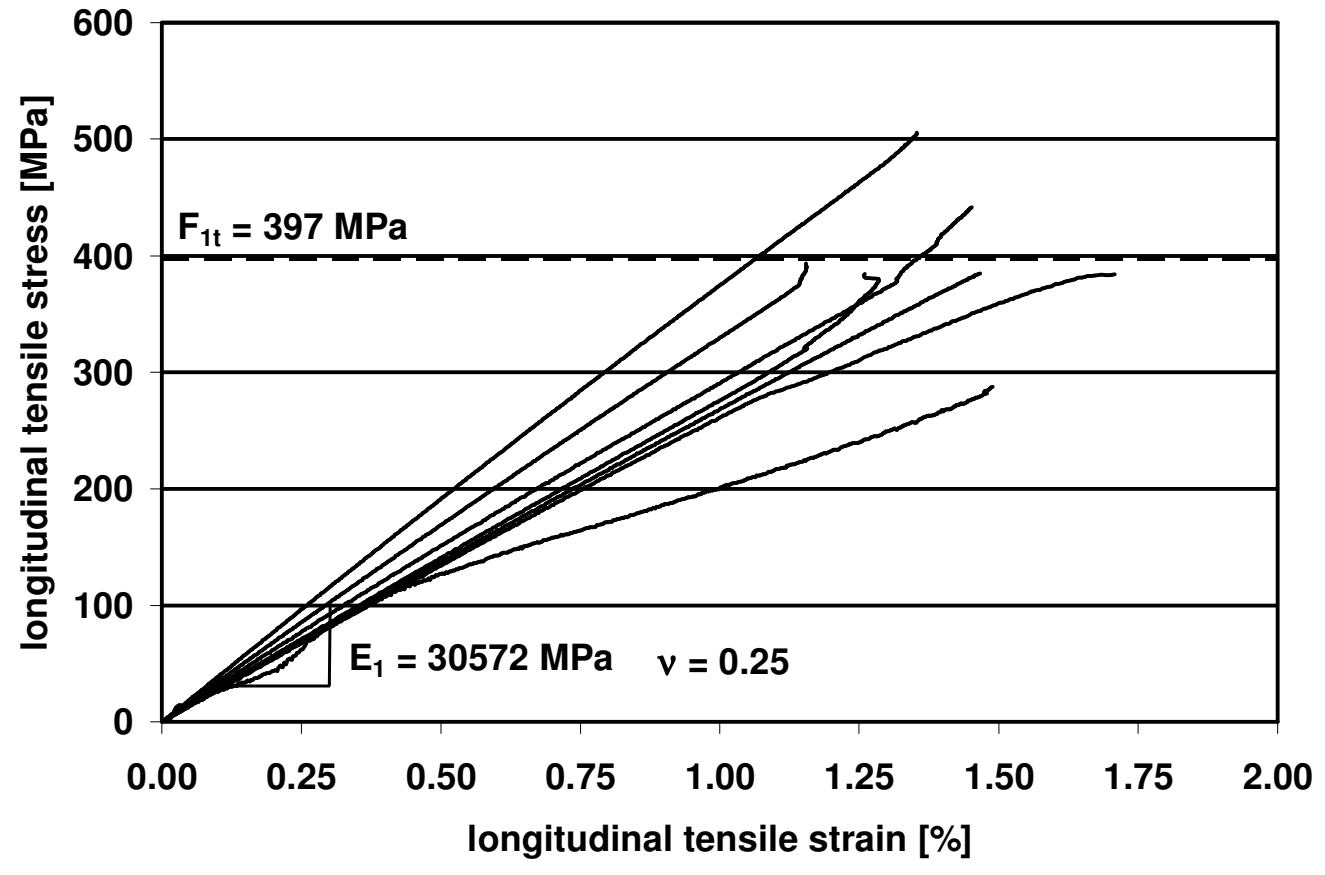

Figure 3.4 Tensile test data in the longitudinal direction for specimens containing the self-healing system. 


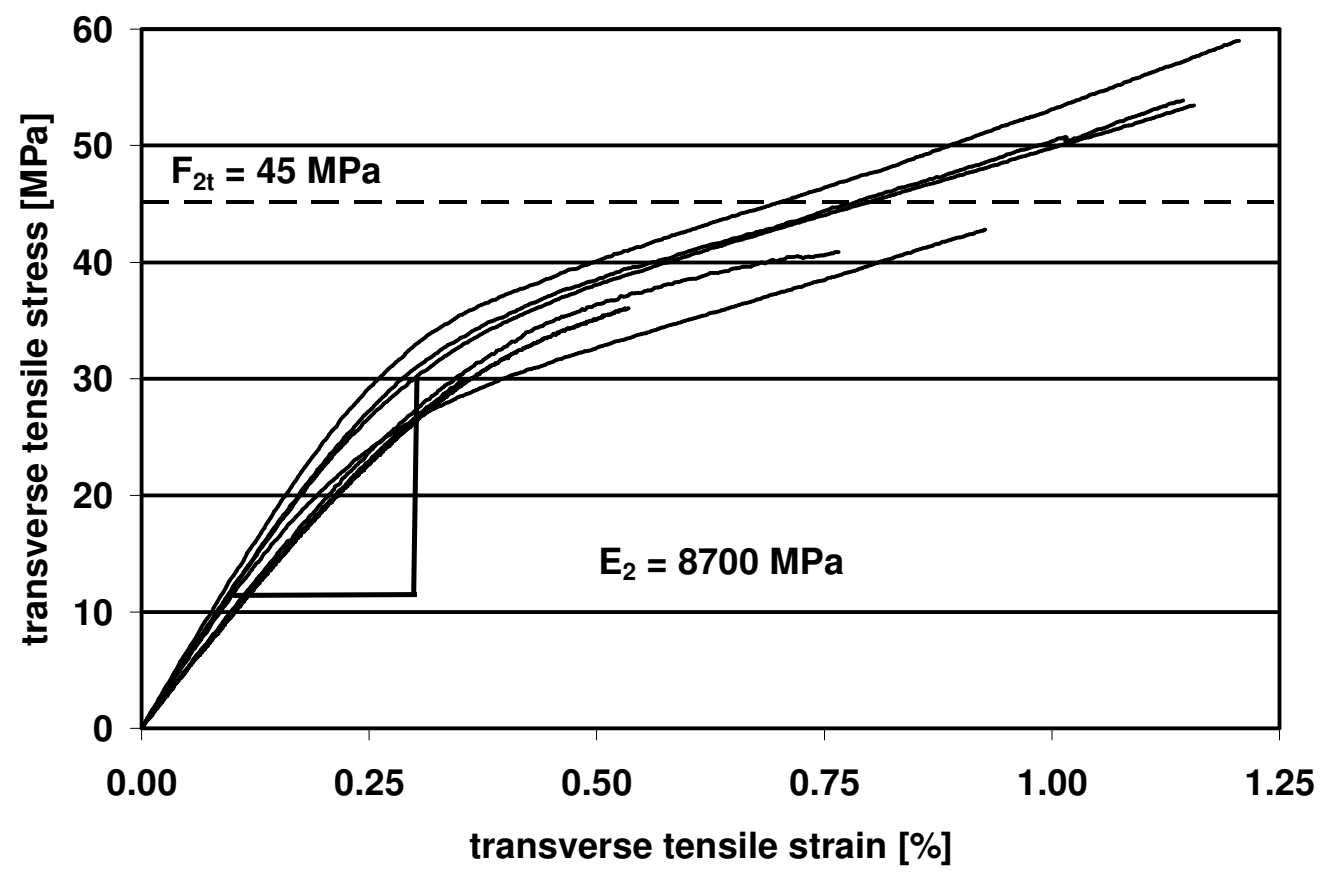

Figure 3.5 Tensile test data in the transverse direction for specimens containing the self-healing system.

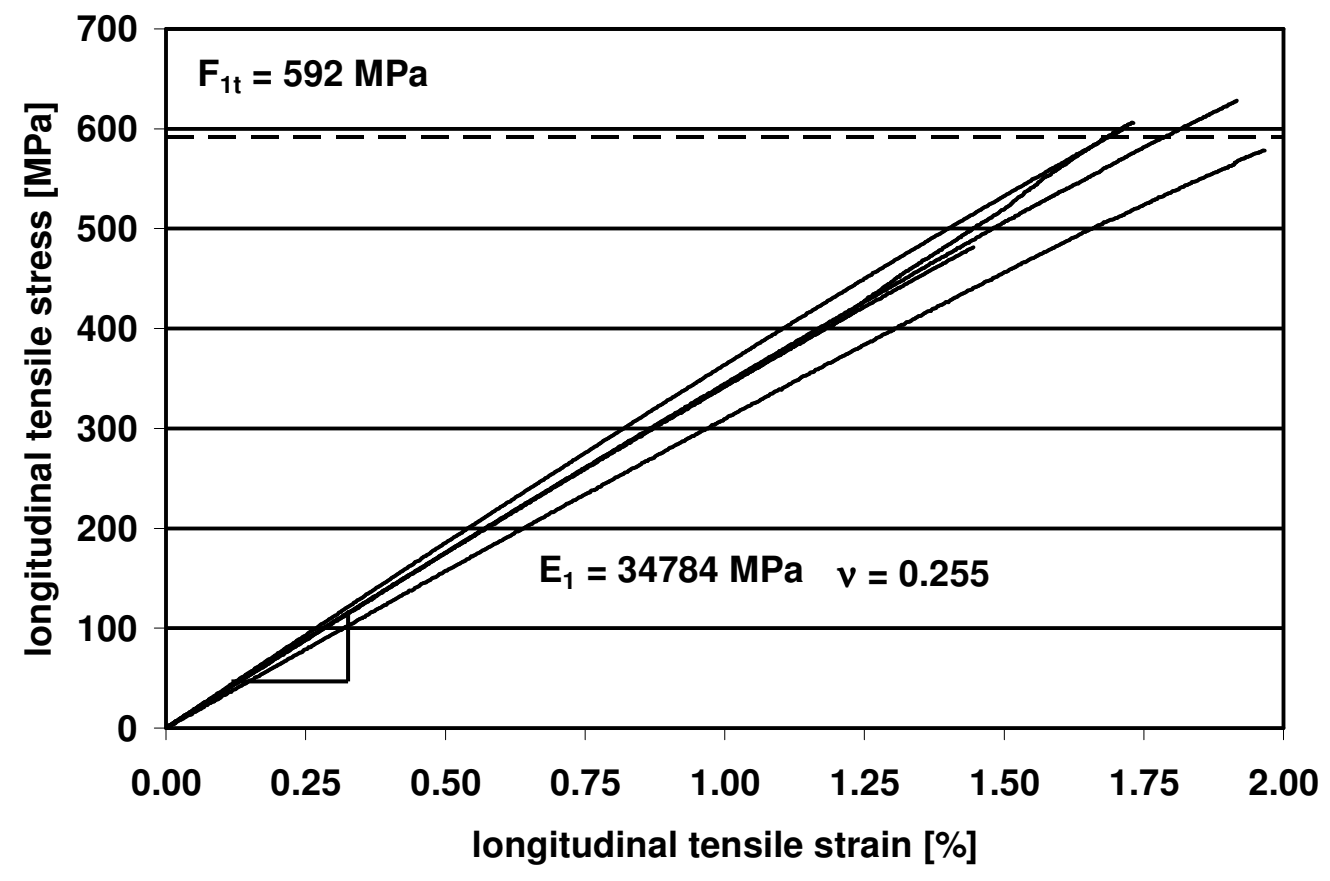

Figure 3.6 Tensile test data in the longitudinal direction for specimens not containing the self-healing system. 


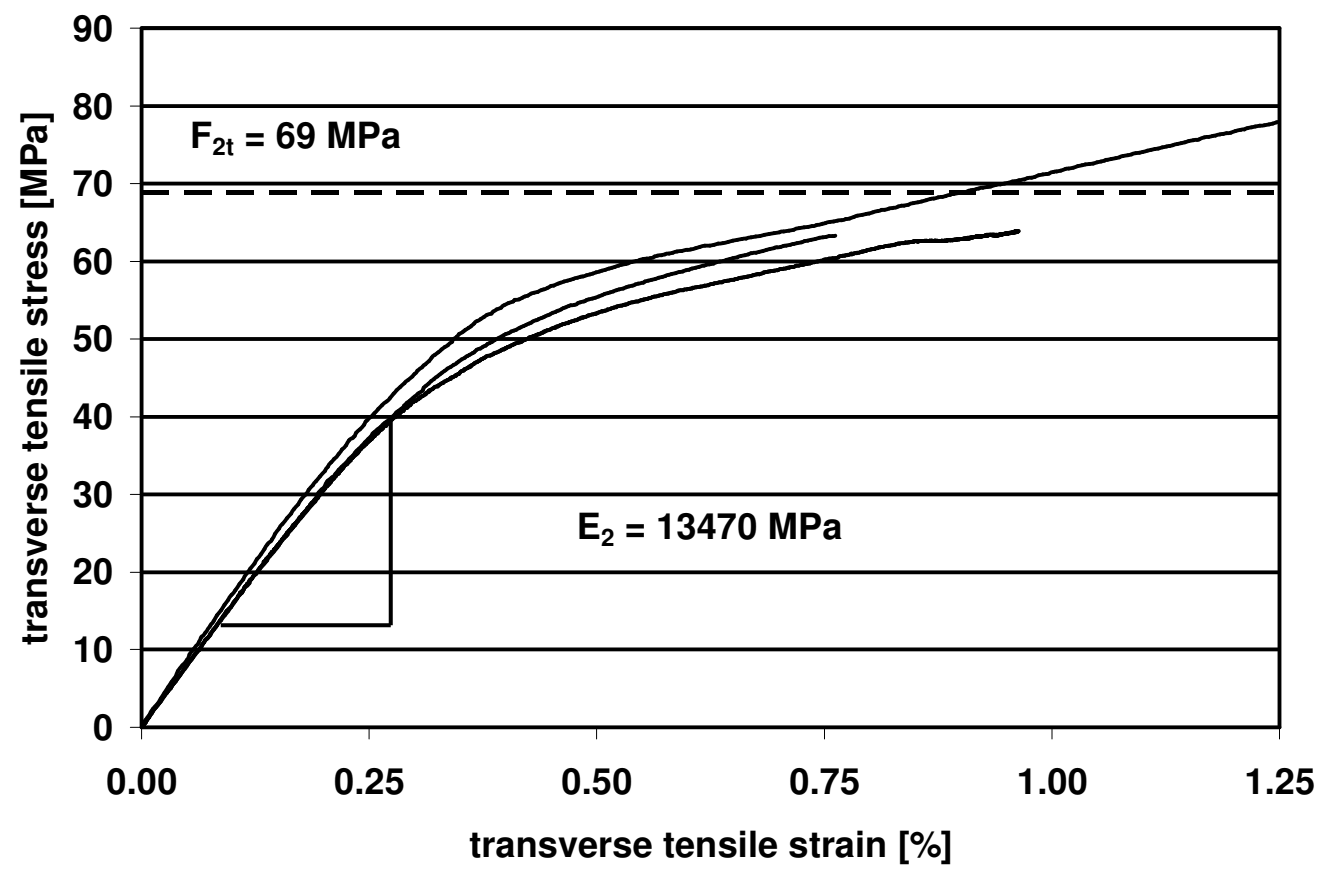

Figure 3.7 Tensile test data in the transverse direction for specimens not containing the self-healing system.
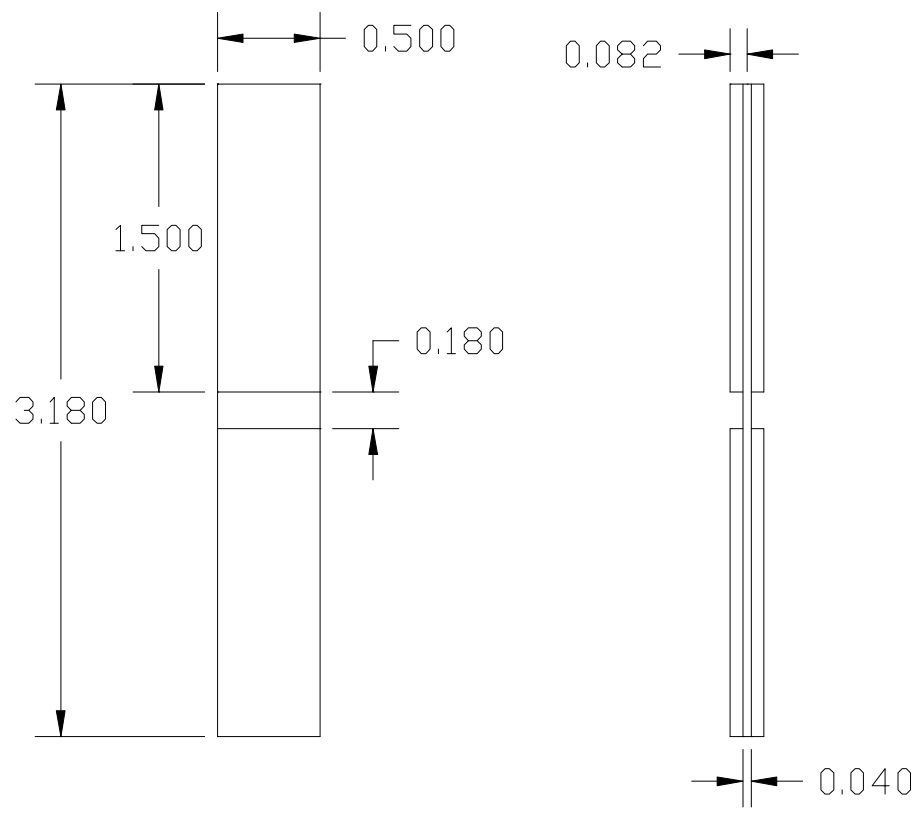

Figure 3.8 SACMA SRM 1R-94 compression test specimen (all dimensions are given in inches). 


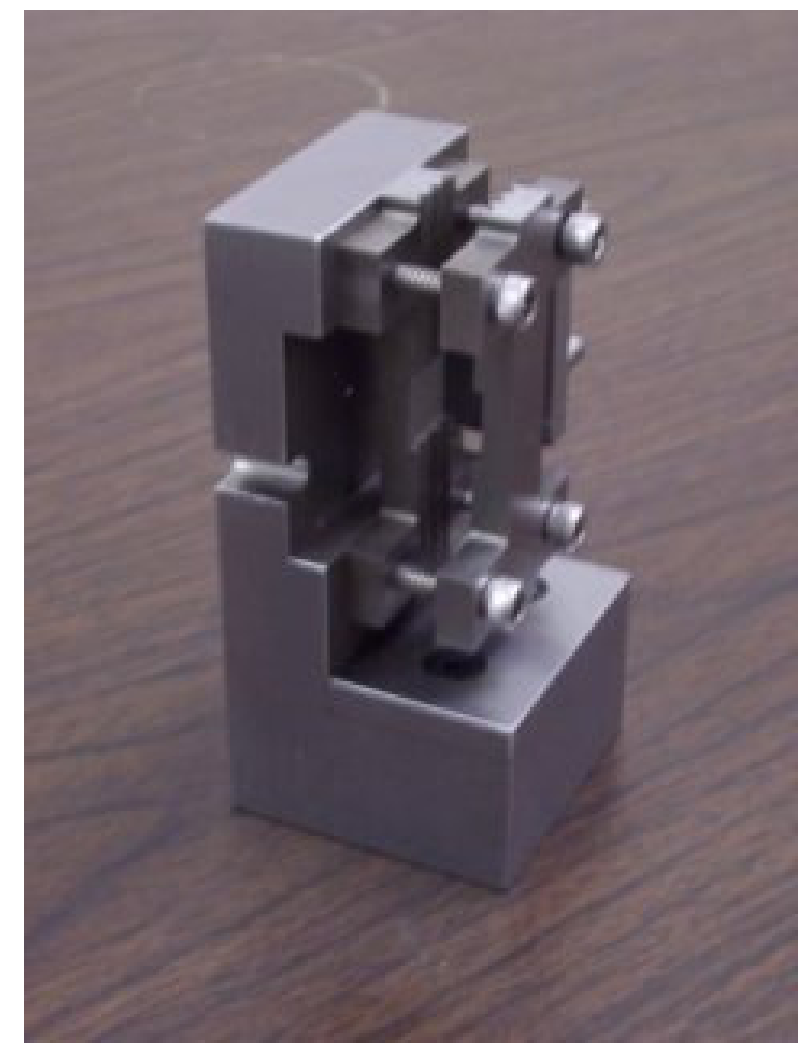

Figure 3.9 SACMA SRM 1R-94 compression test fixture.

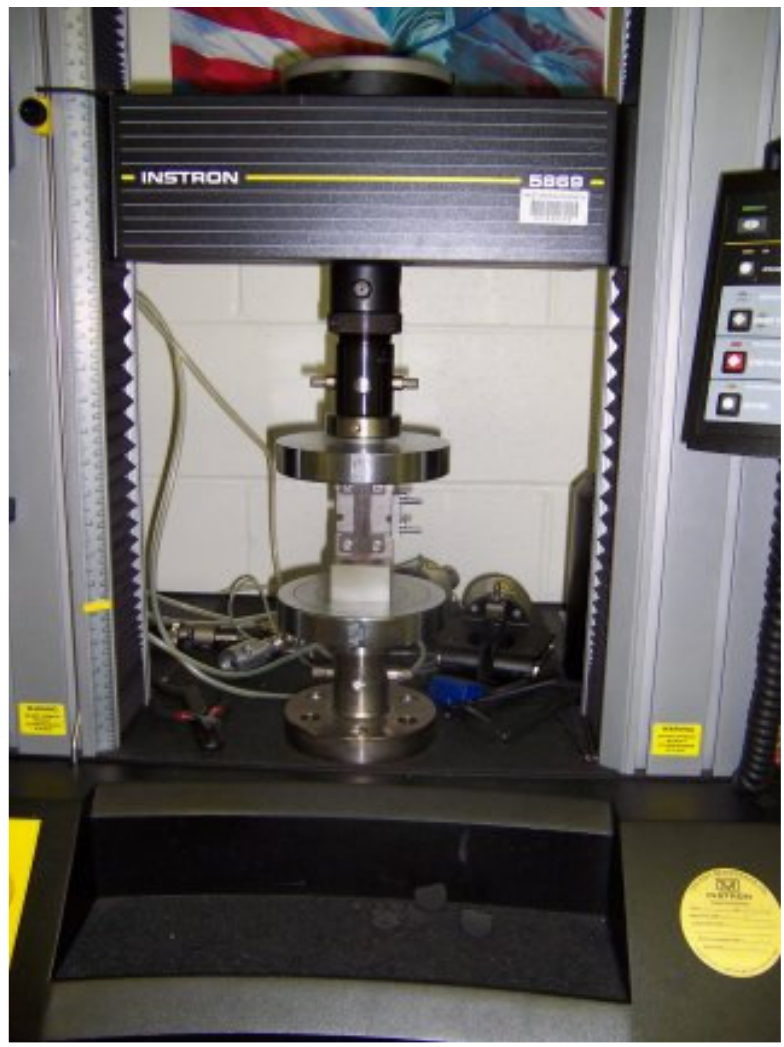

Figure 3.10 Instron testing machine with SACMA compression fixture. 


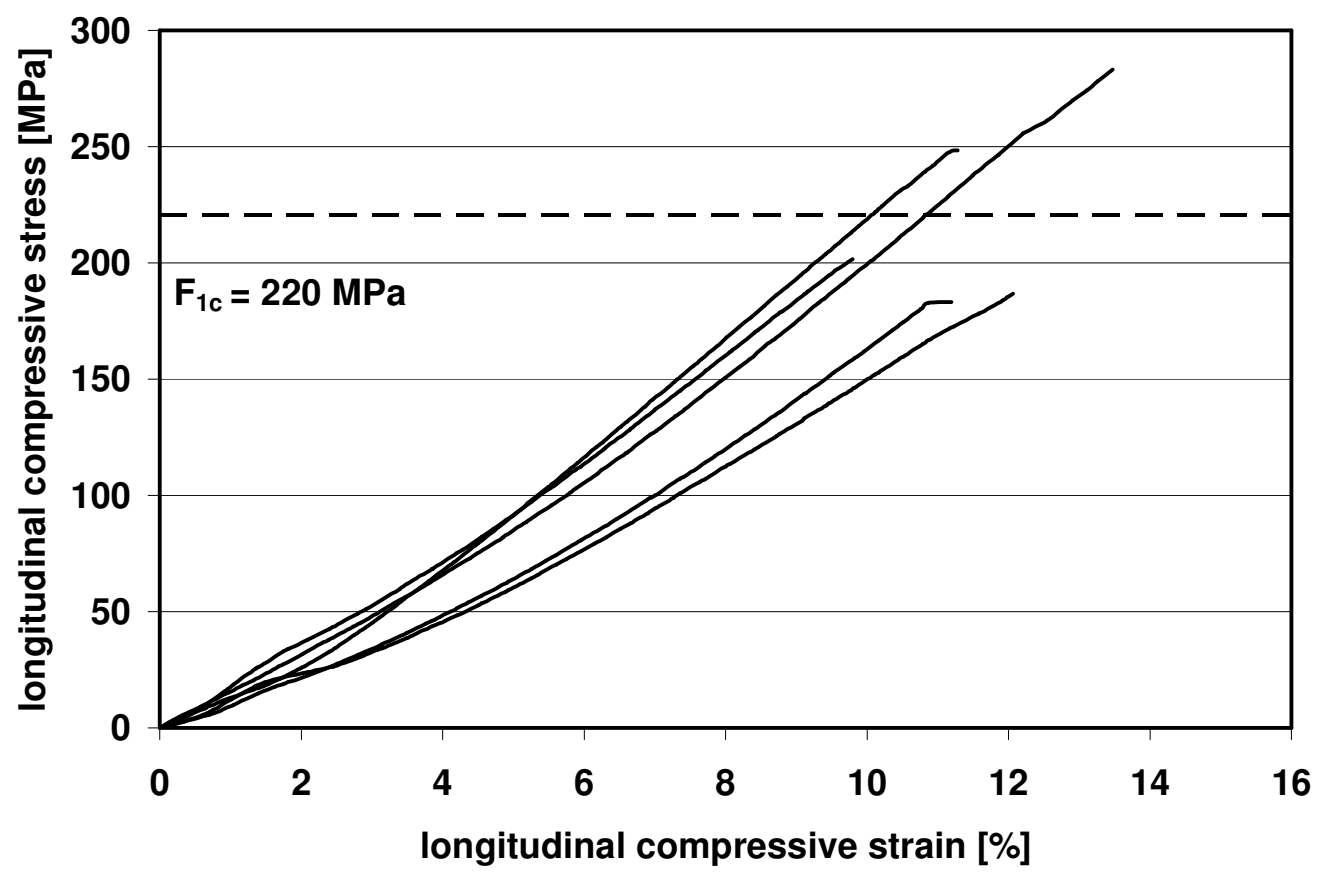

Figure 3.11 Compression test data for specimens containing the self-healing system in the longitudinal direction.

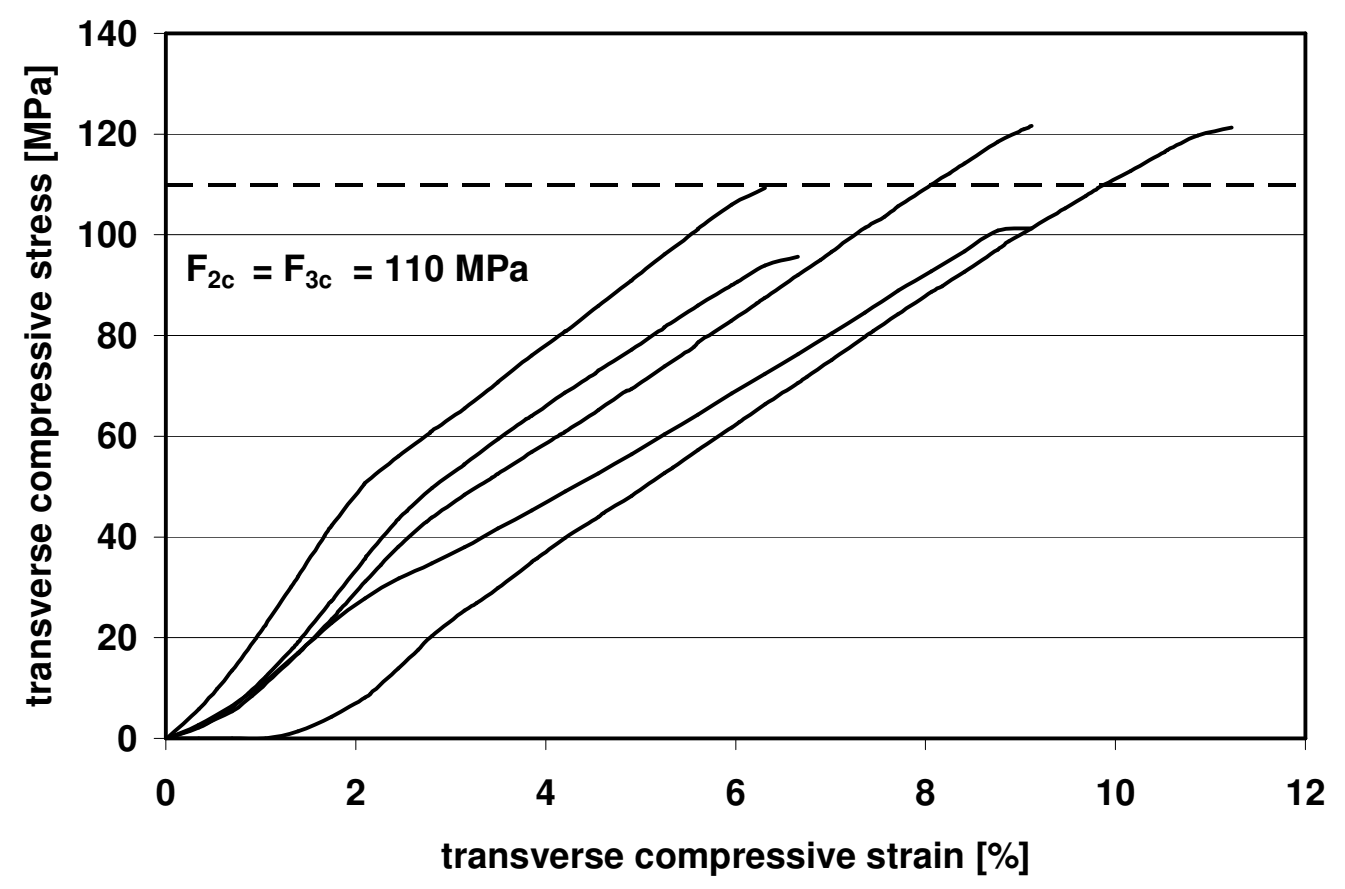

Figure 3.12 Compression test data for specimens containing the self-healing system in the transverse direction. 


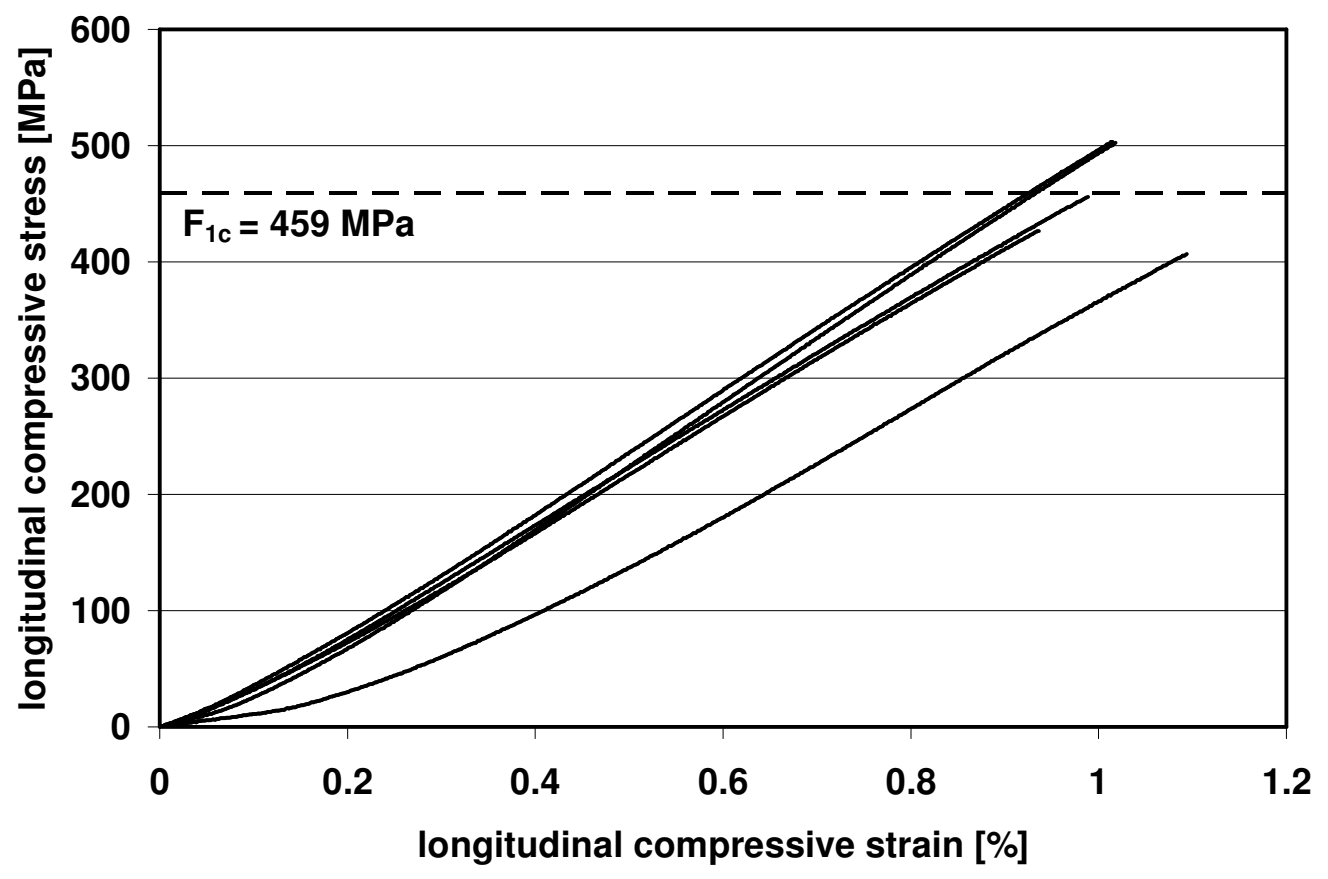

Figure 3.13 Compression test data for specimens not containing the self-healing system in the longitudinal direction.

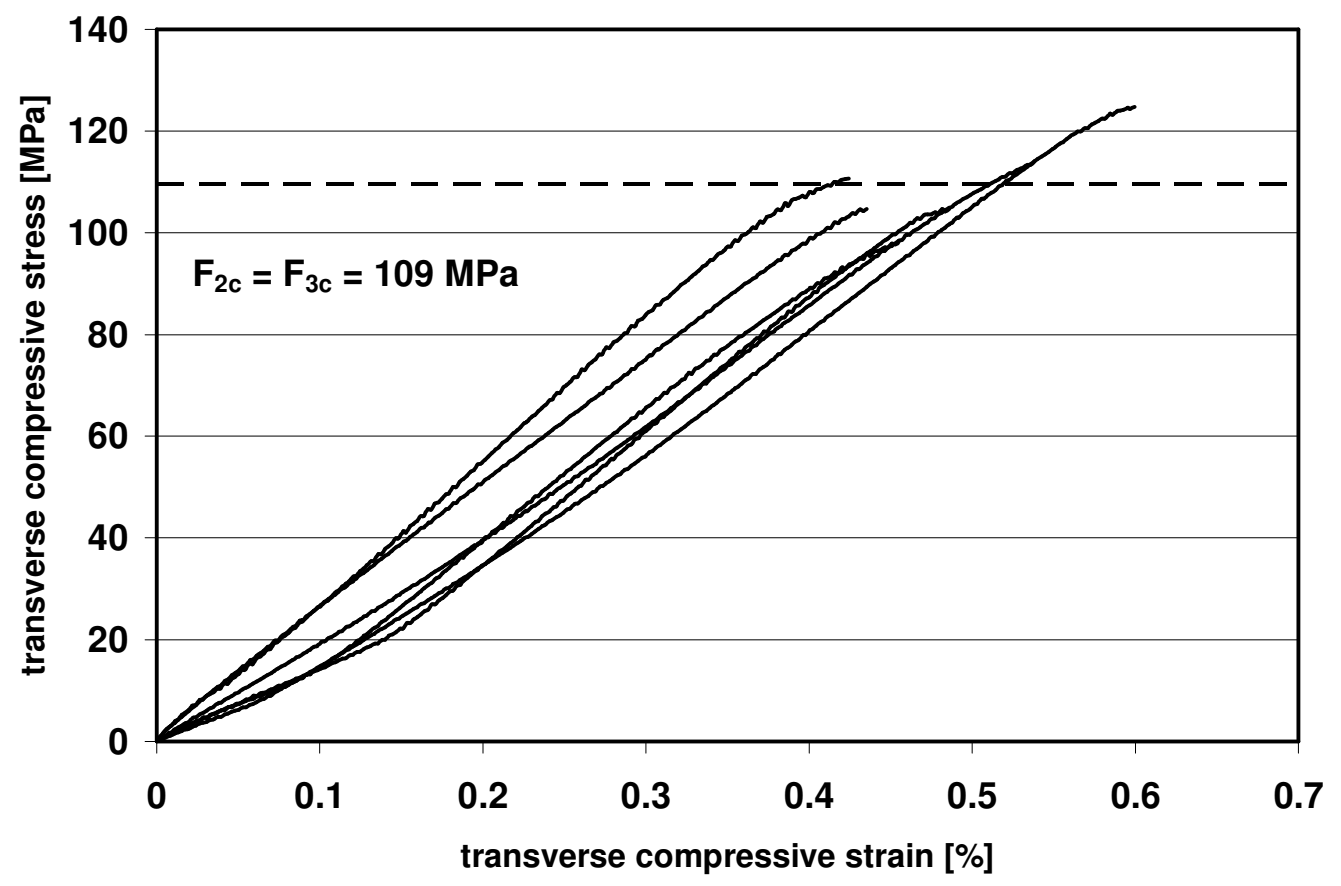

Figure 3.14 Compression test data for specimens not containing the self-healing system in the transverse direction. 

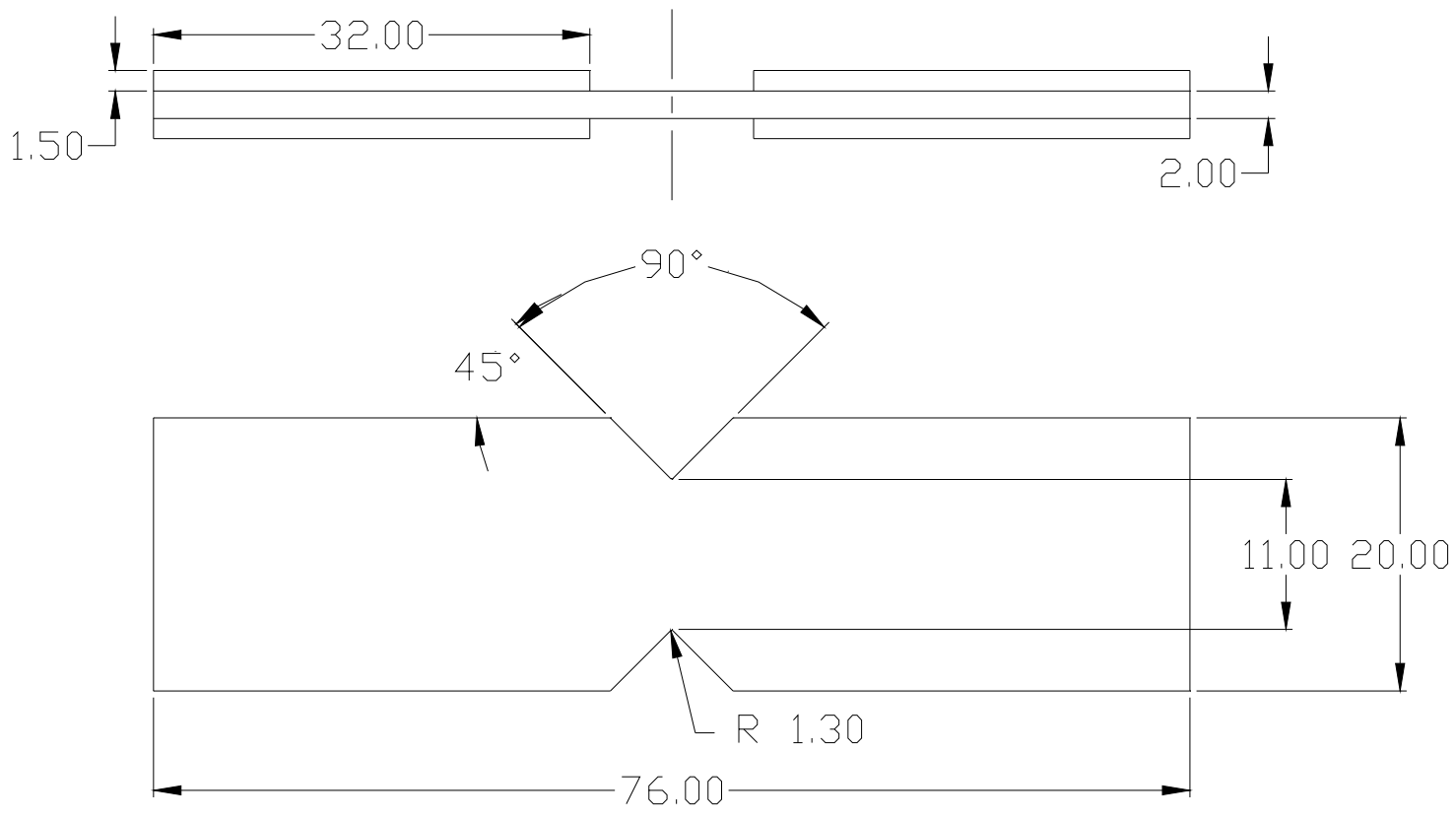

Figure 3.15 Iosipescu test specimen (all dimensions are given in mm). 

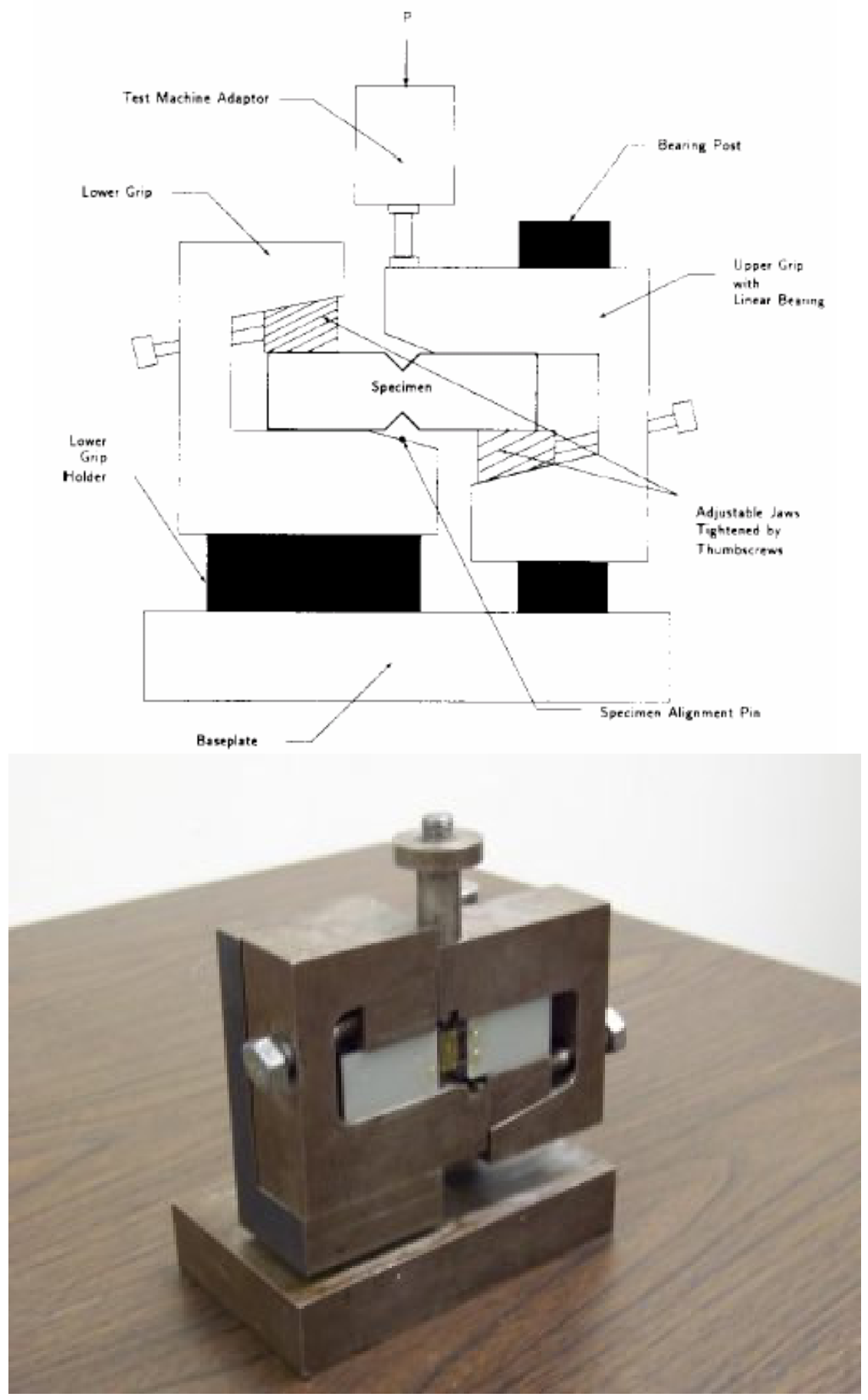

Figure 3.16 Iosipescu test fixture diagram and picture of test fixture. 


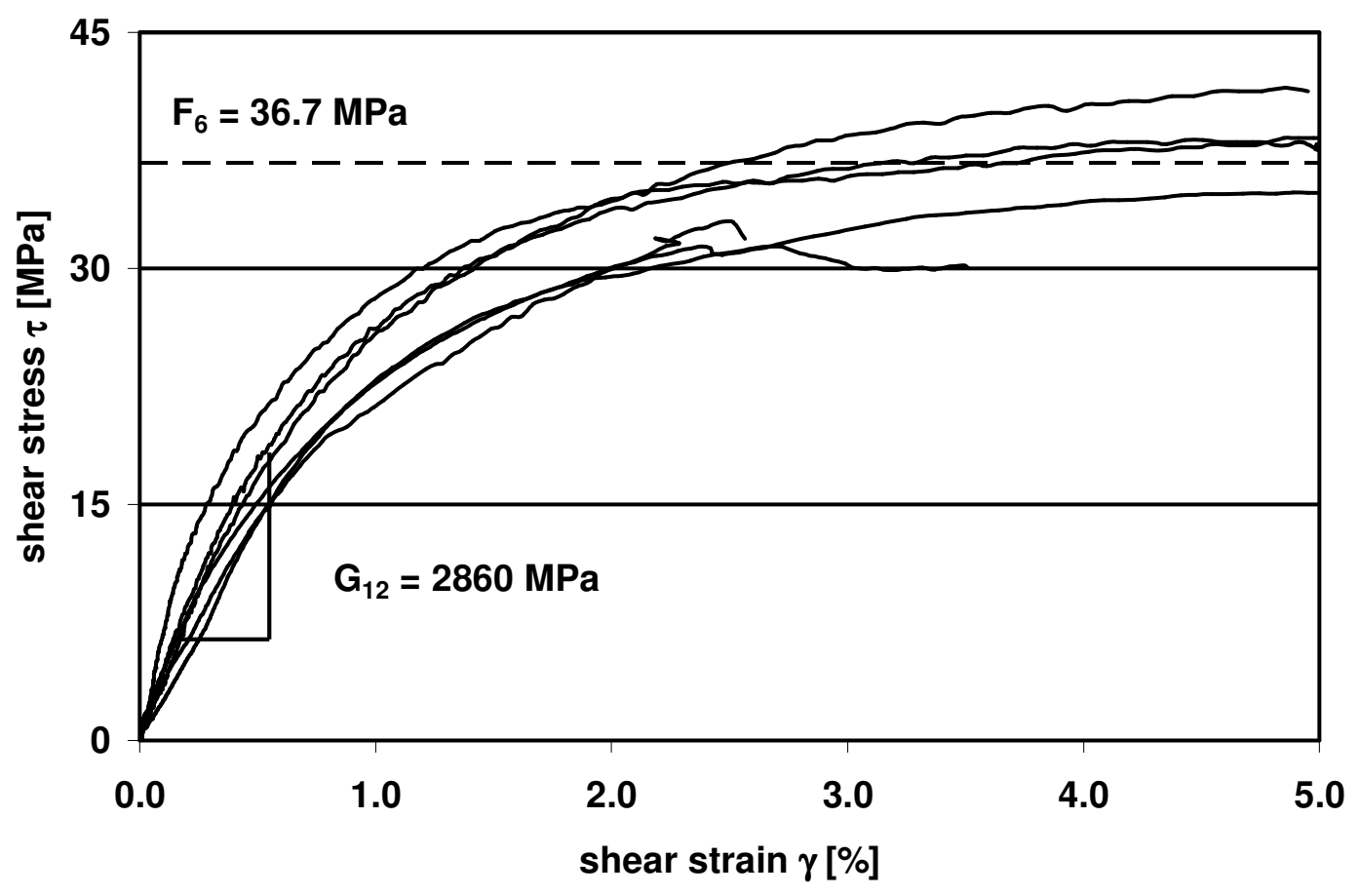

Figure 3.17 Shear data for specimens containing the self-healing system in the ' $1-2$ direction'.

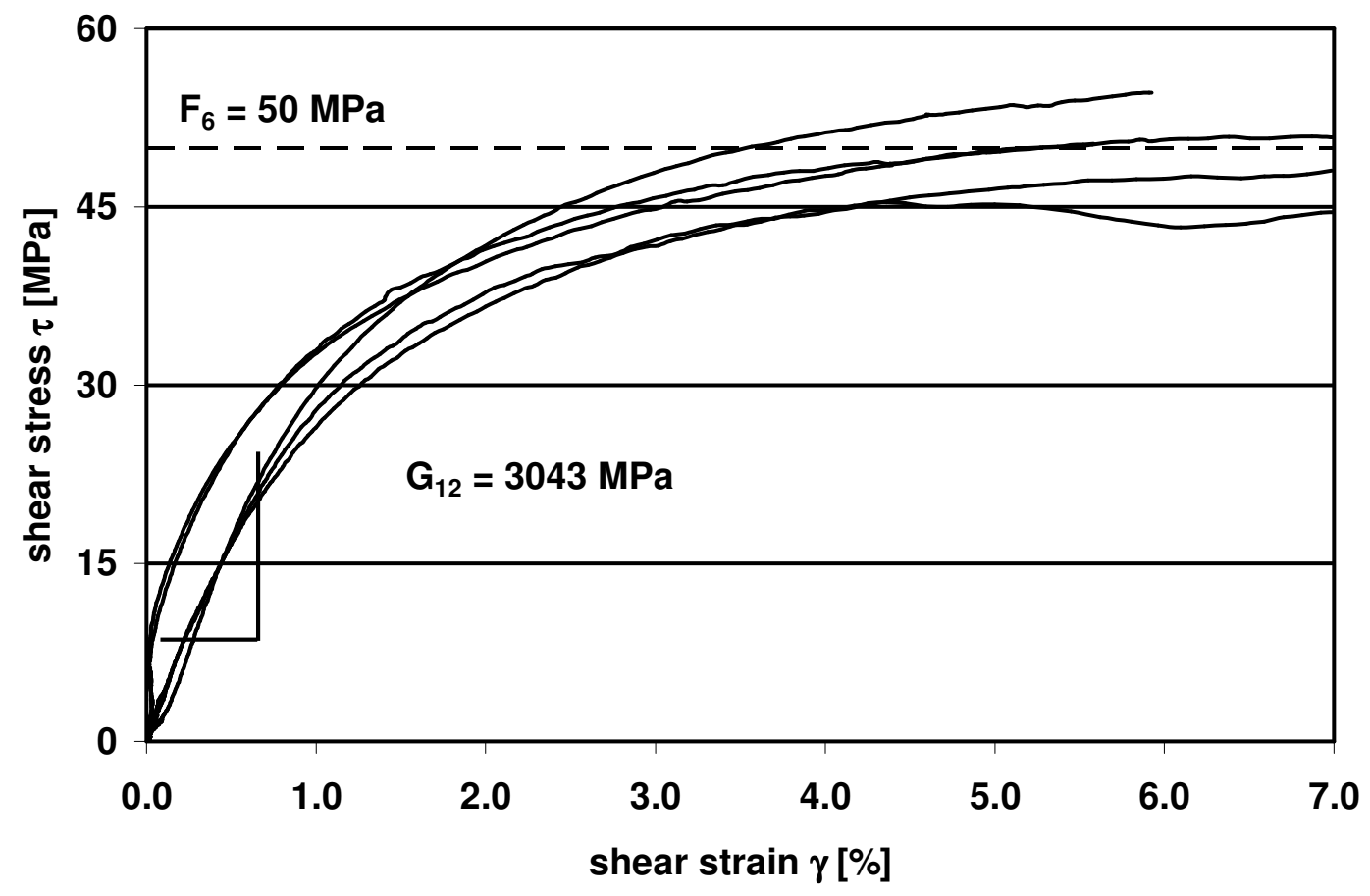

Figure 3.18 Shear data for specimens not containing the self-healing system in the '1-2 direction'. 


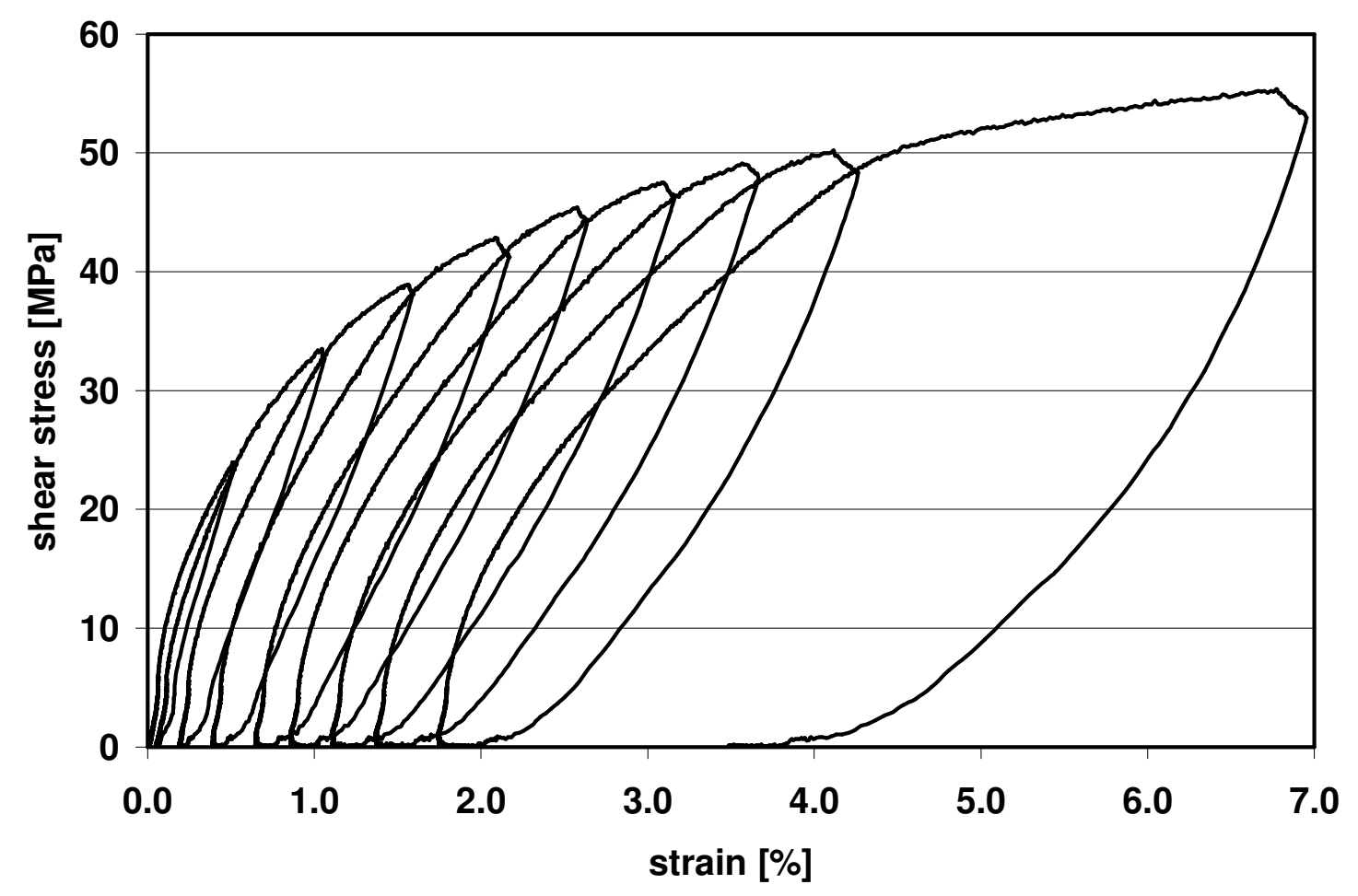

Figure 3.19 Cyclic loading of shear specimen without self-healing system.

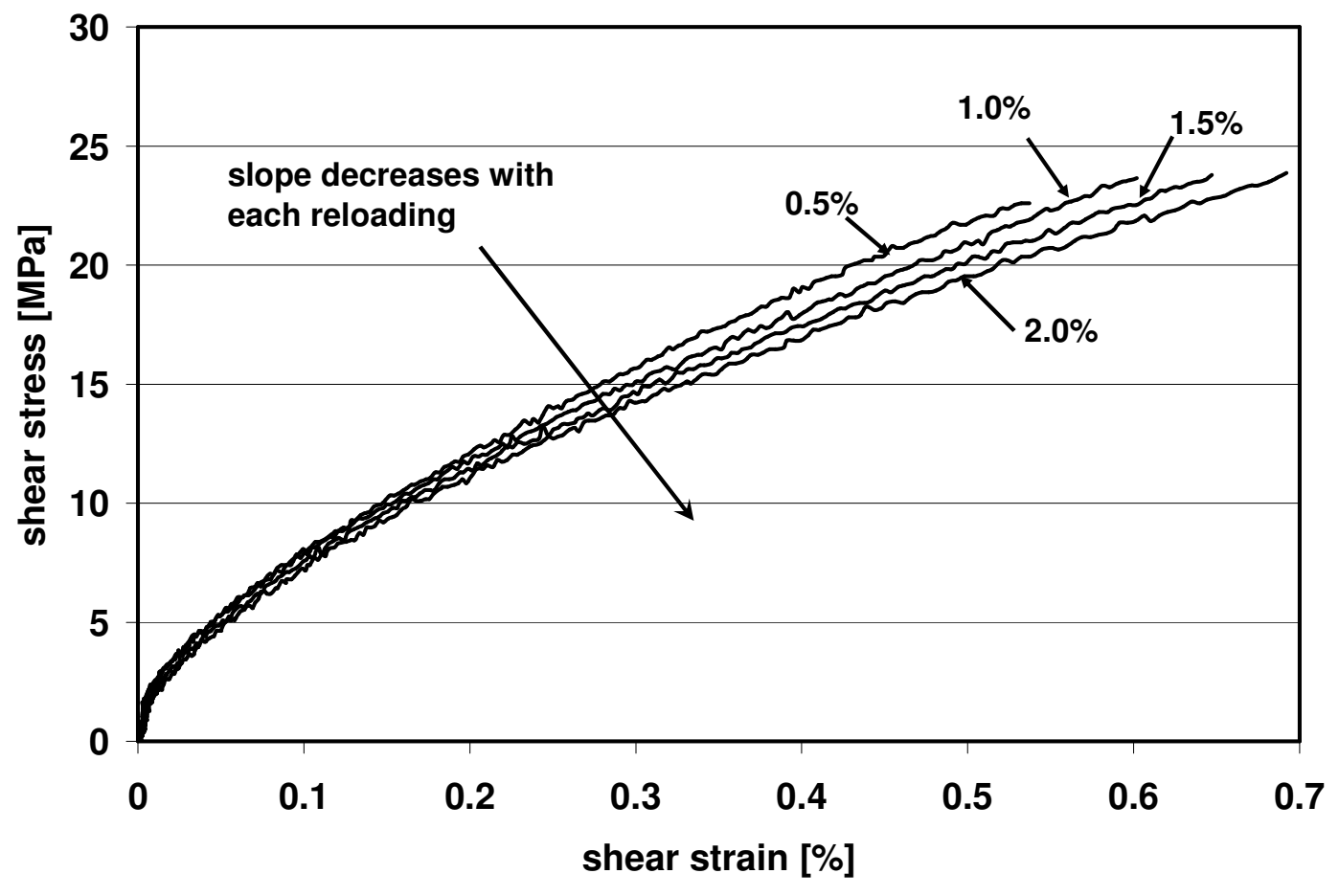

Figure 3.20 Comparison of the shear loading curves from the specimen in Figure 3.19 moved to the origin. 


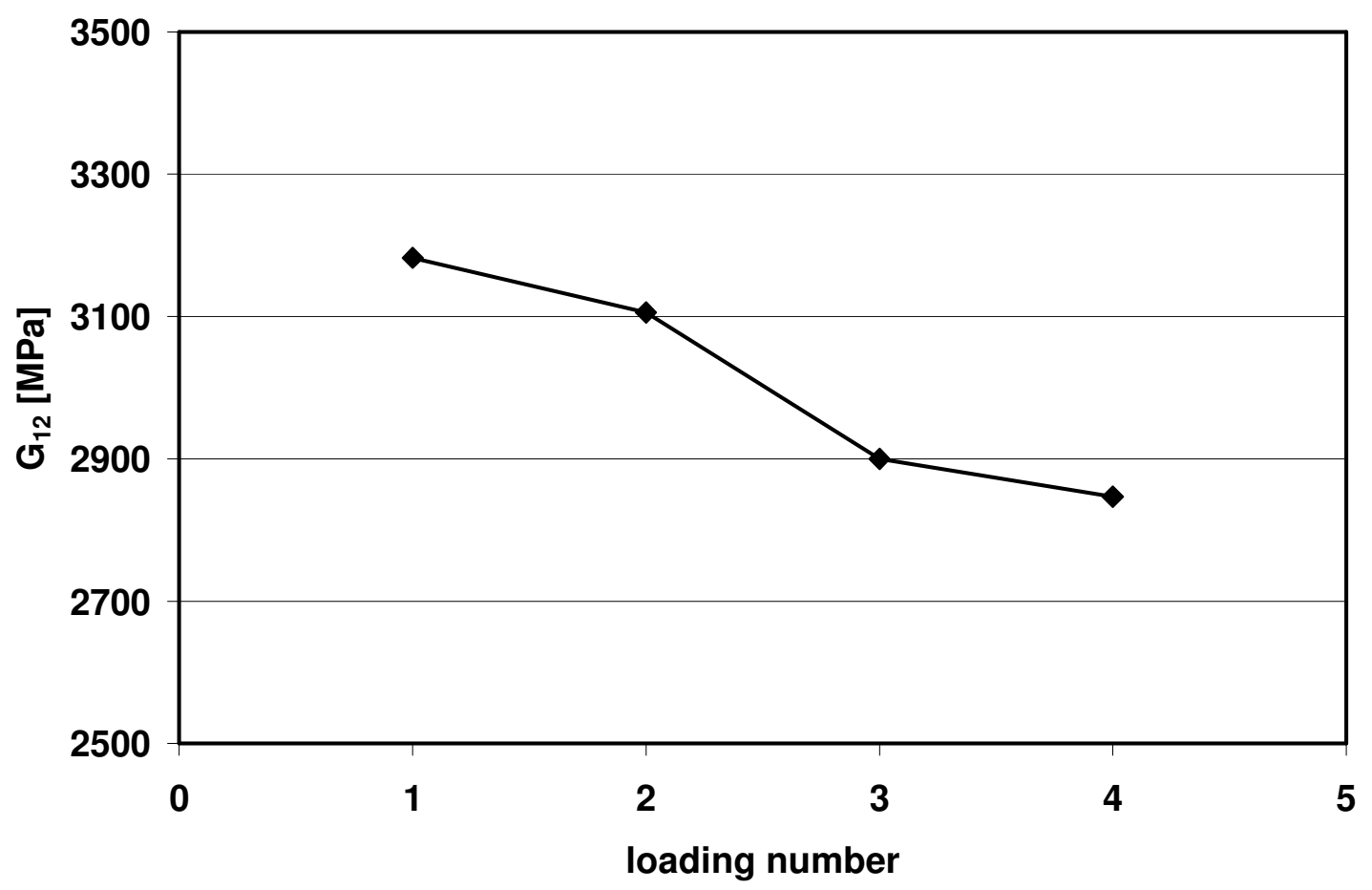

Figure 3.21 Cyclic shear slopes $\left(G_{12}\right)$ vs. loading number of specimen in Figure 3.19 and Figure 3.20. Notice that with each reloading the slope decreases.

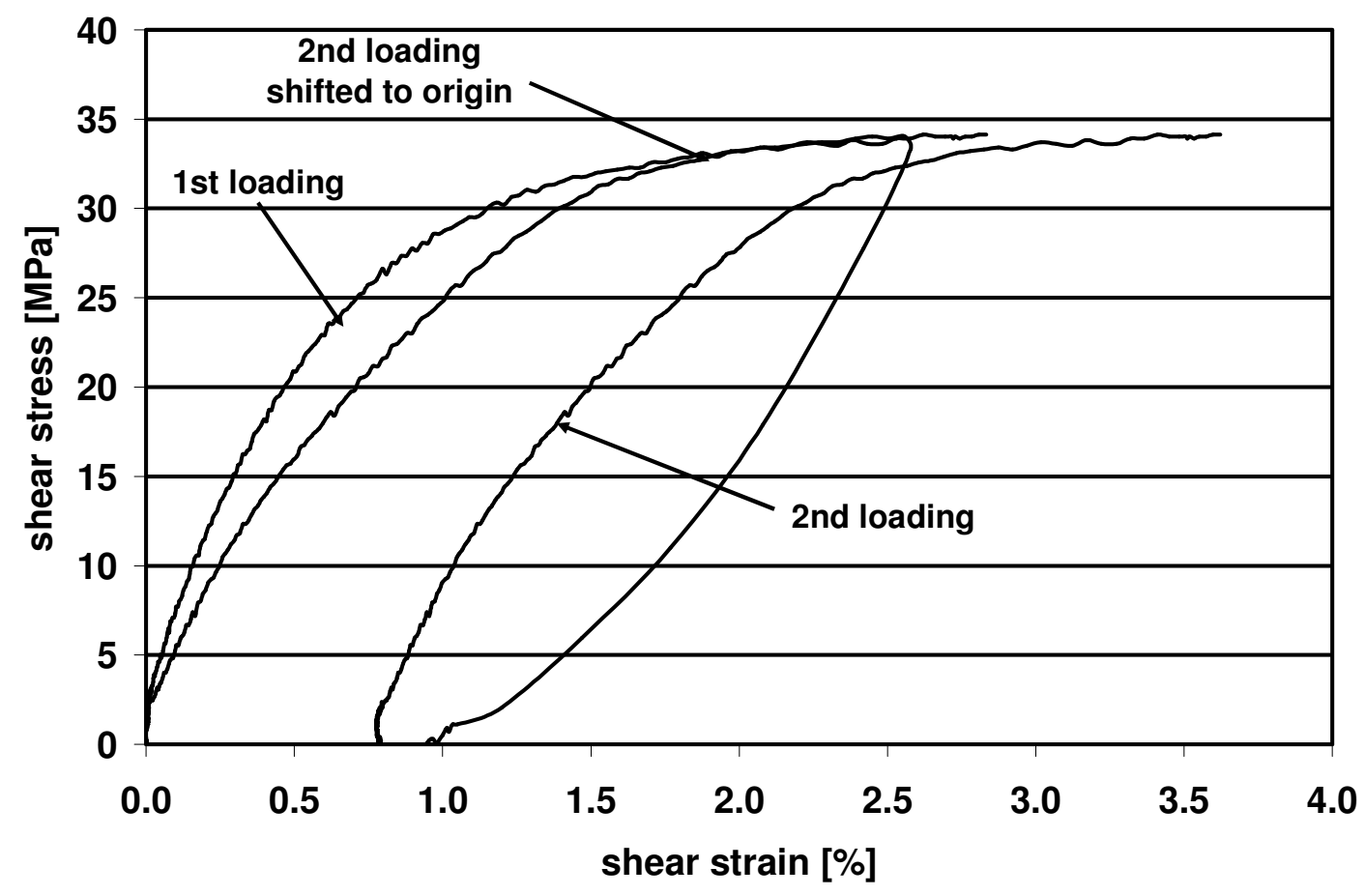

Figure 3.22 Specimen with self-healing system loaded to $2.5 \%$ strain unloaded, not allowed to heal, then reloaded. 


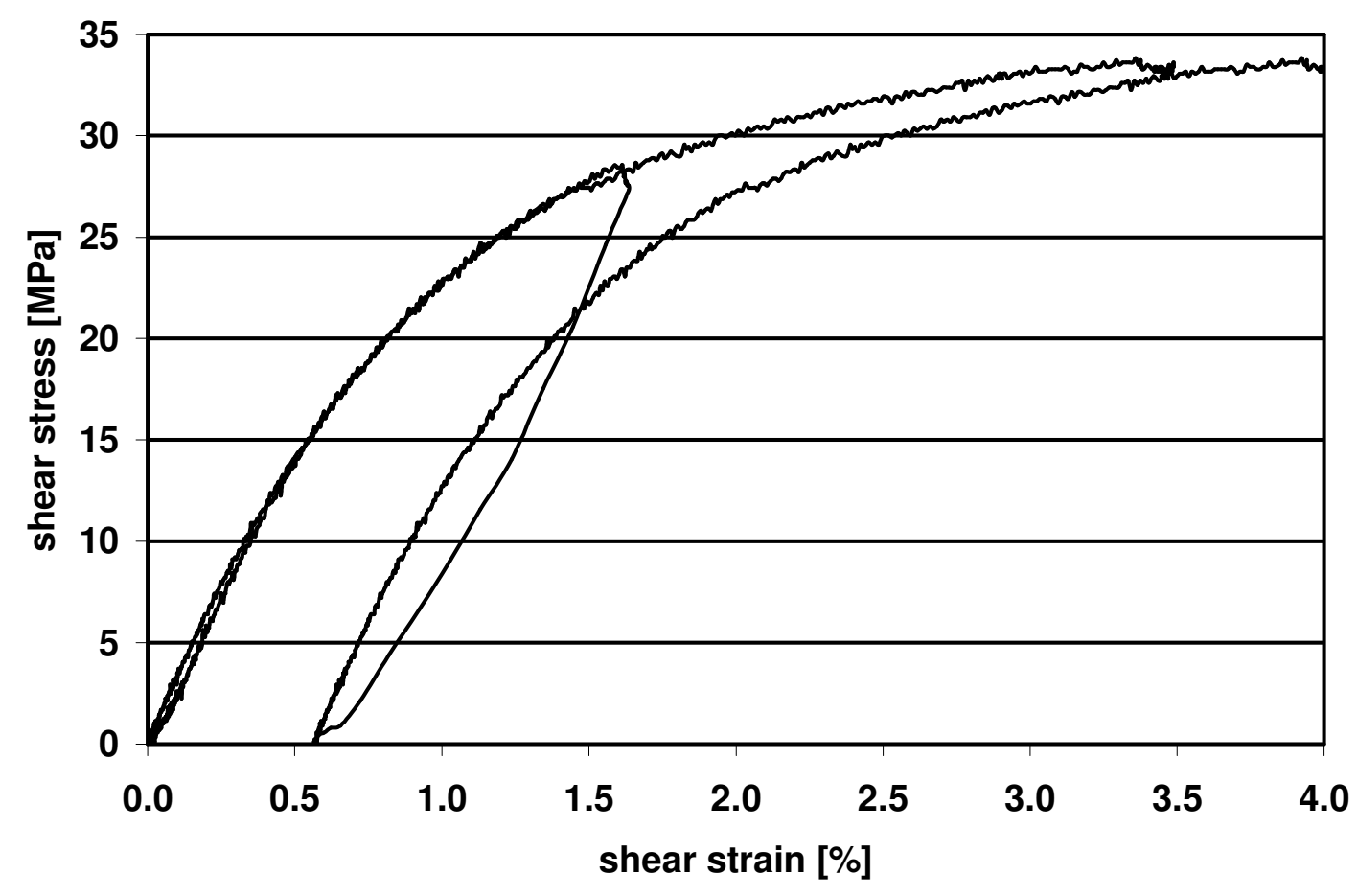

Figure 3.23 Specimen with self-healing system loaded to $1.5 \%$ strain, allowed to heal (48 hrs), then reloaded.

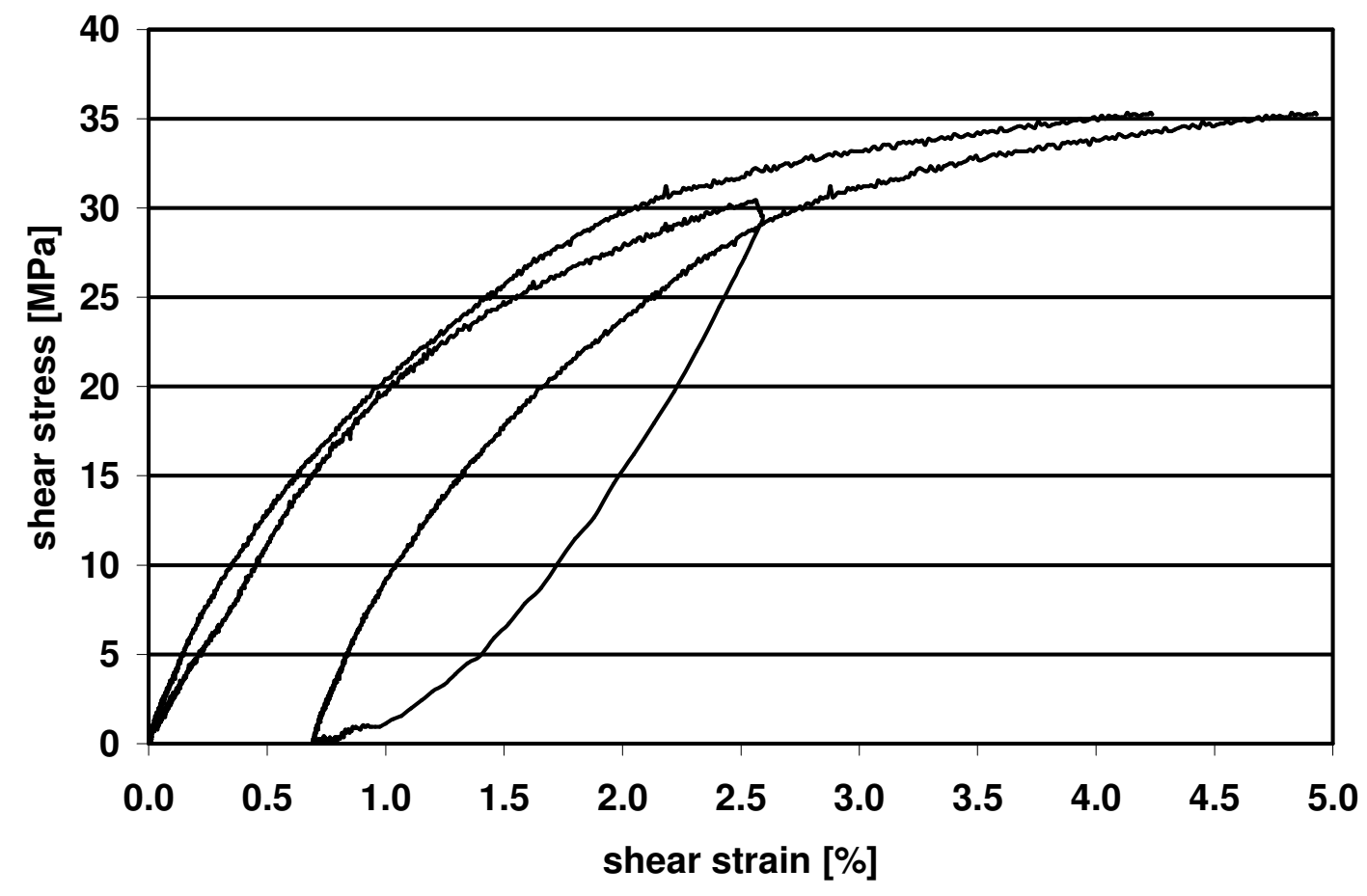

Figure 3.24 Specimen with self-healing system loaded to $2.5 \%$ strain, allowed to heal (48 hrs), then reloaded. 


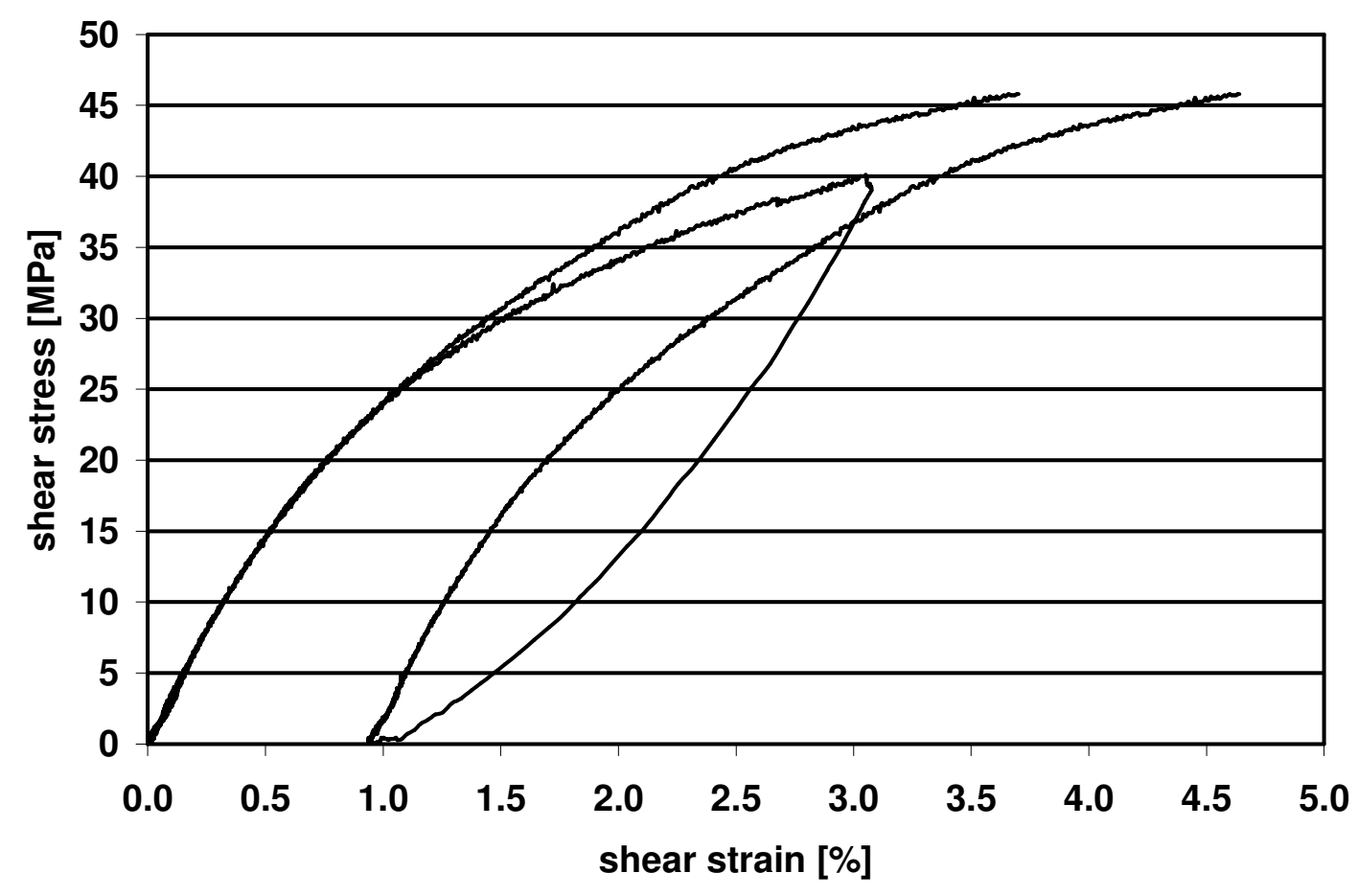

Figure 3.25 Specimen with self-healing system loaded to $3.0 \%$ strain, allowed to heal (48 hrs), then reloaded.

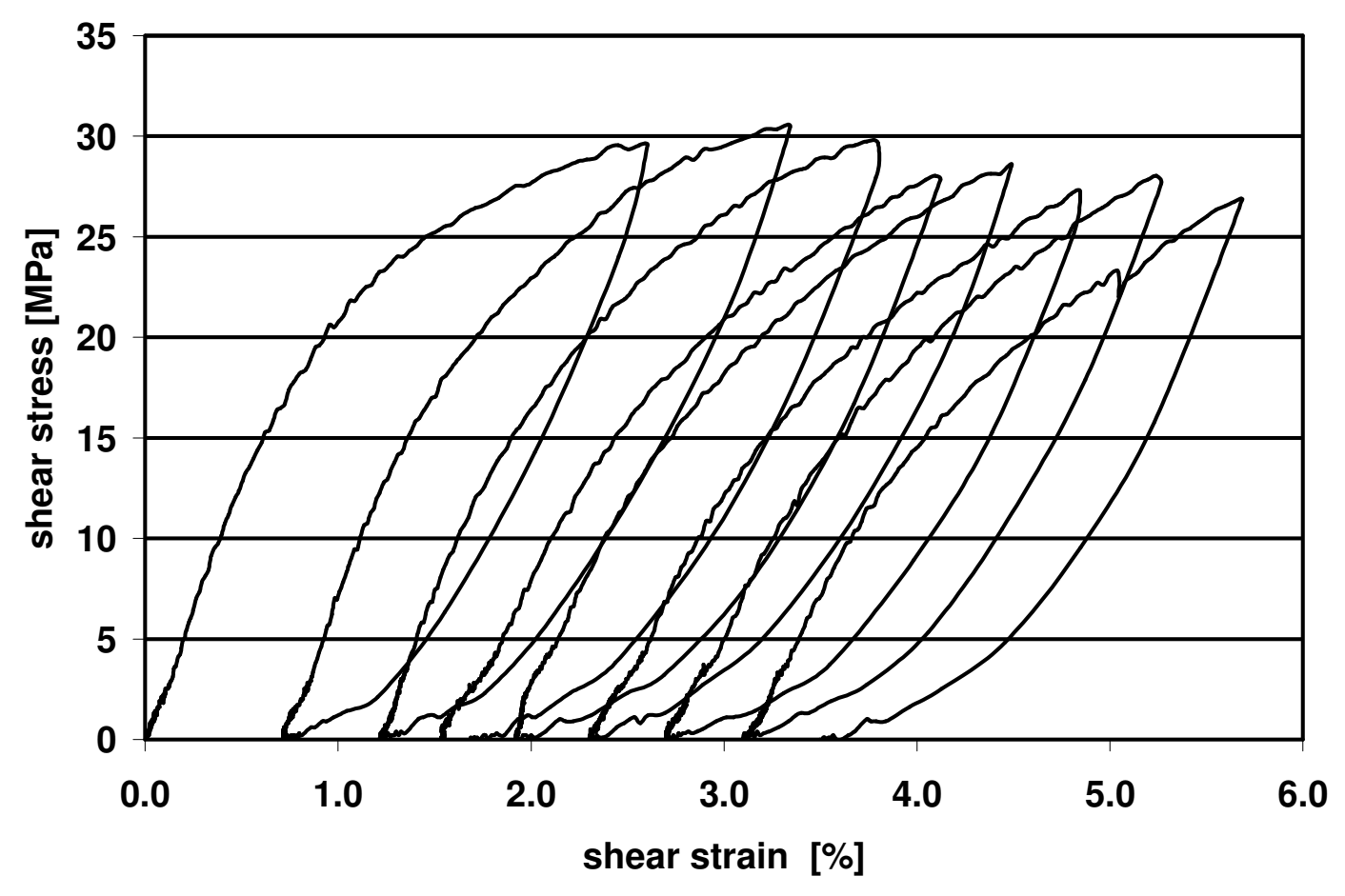

Figure 3.26 Typical cyclic healing shear test. 


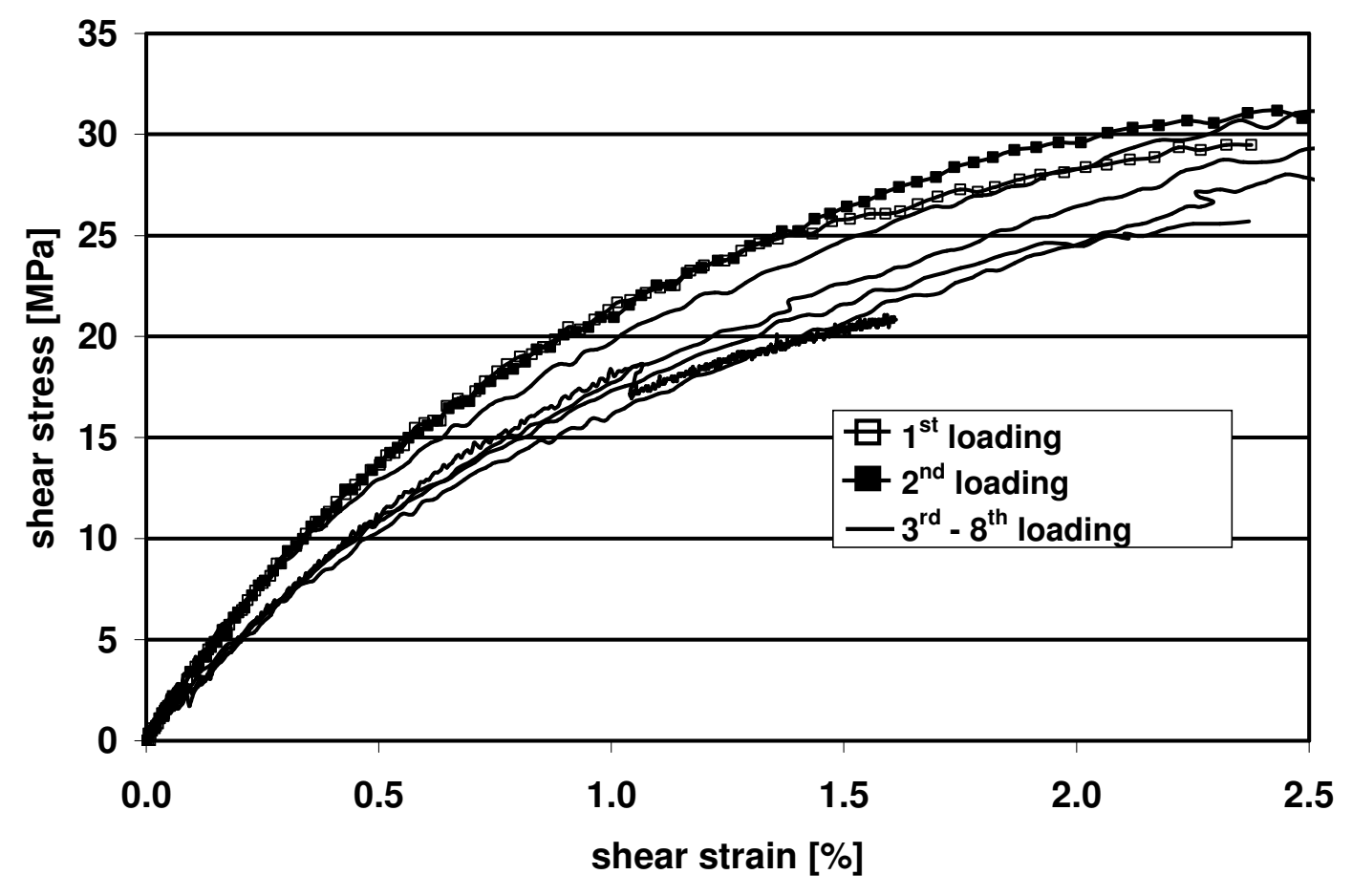

Figure 3.27 Cyclic healing shear testing, all data is shifted to the origin.

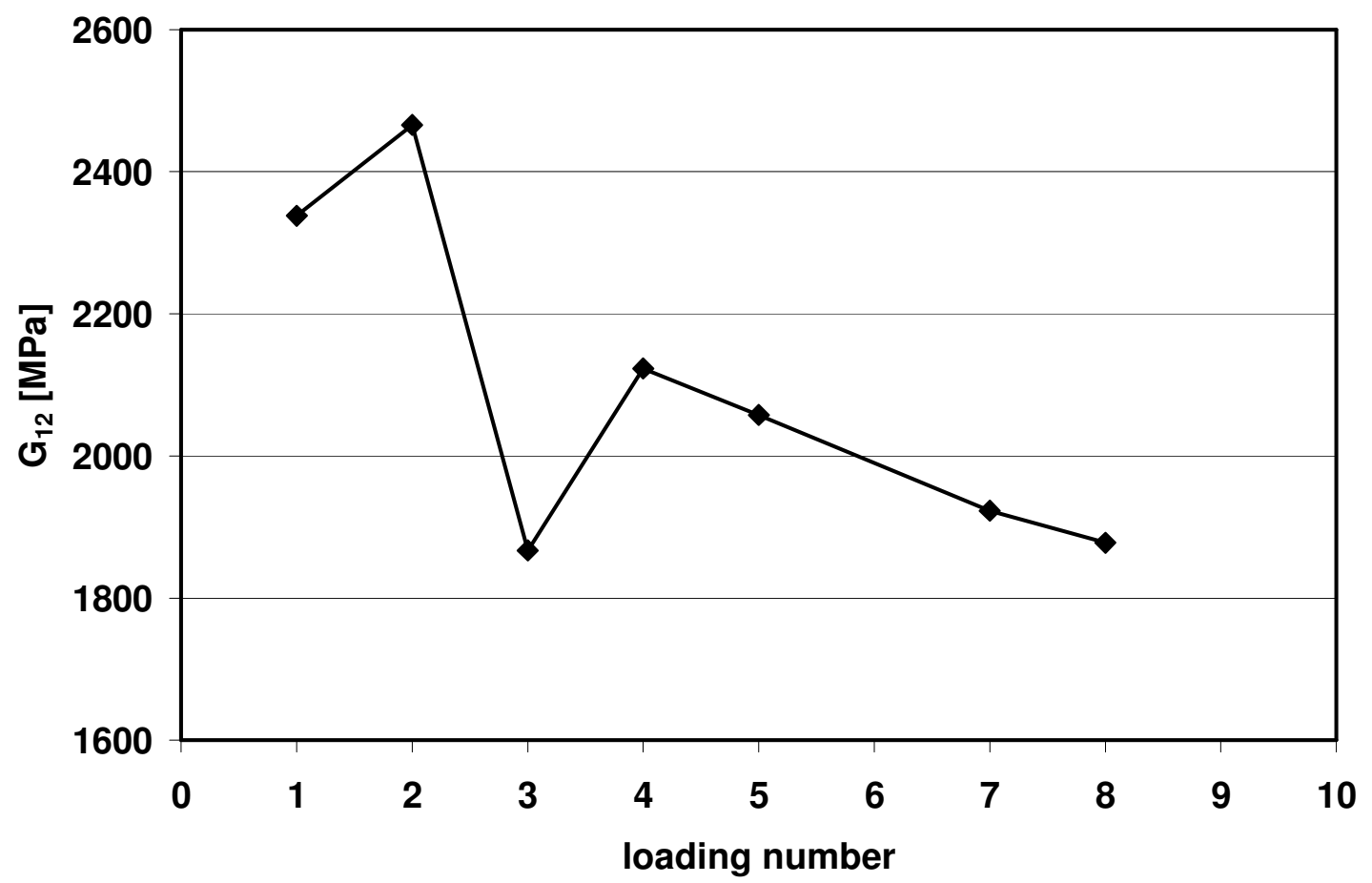

Figure 3.28 Cyclic shear slopes $\left(\mathbf{G}_{12}\right)$ vs. loading number of specimen in Figure 3.27. Notice that with slope each reloading the slope does not necessarily decrease. 


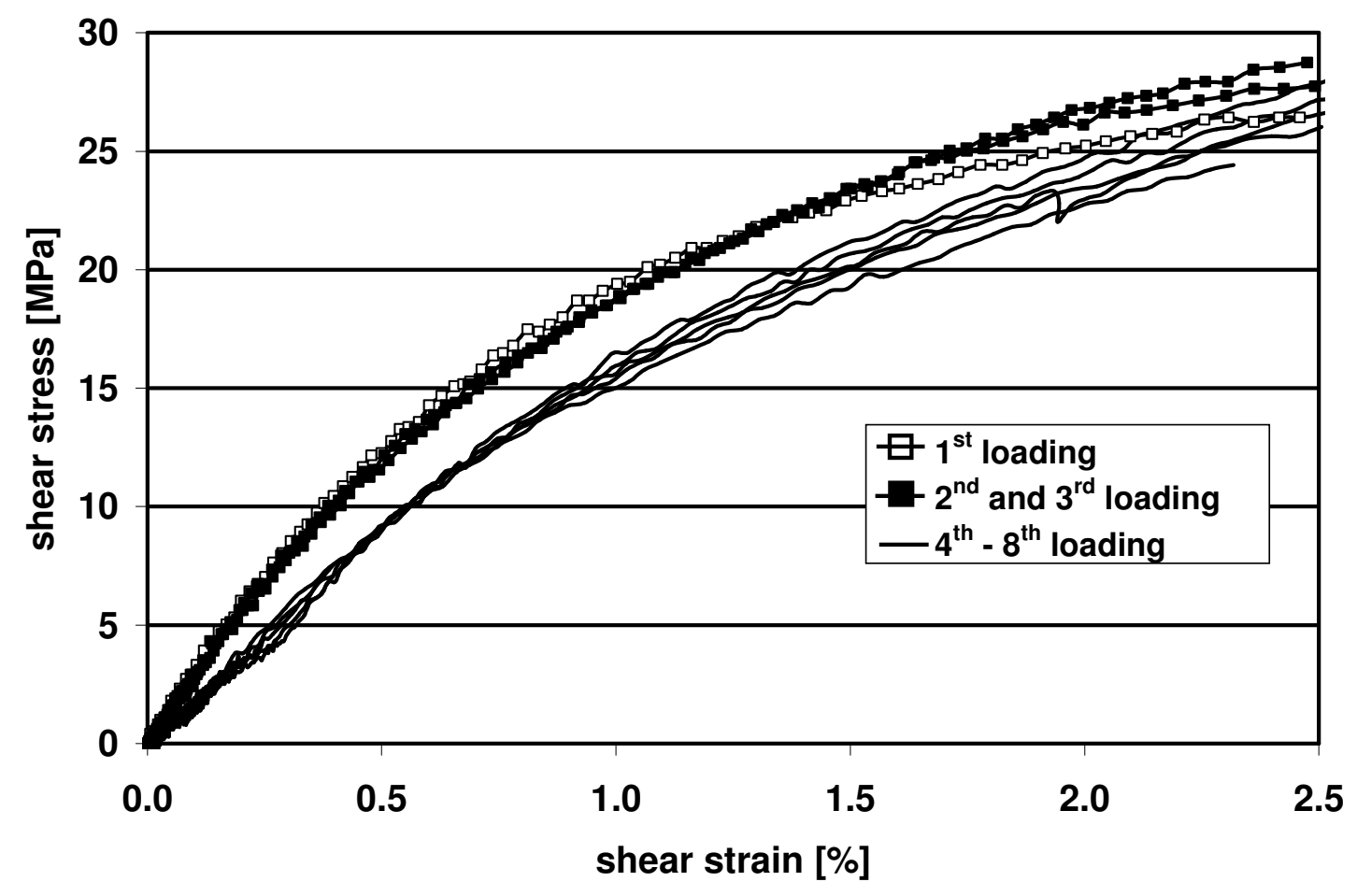

Figure 3.29 Cyclic healing shear testing, all data is shifted to the origin.

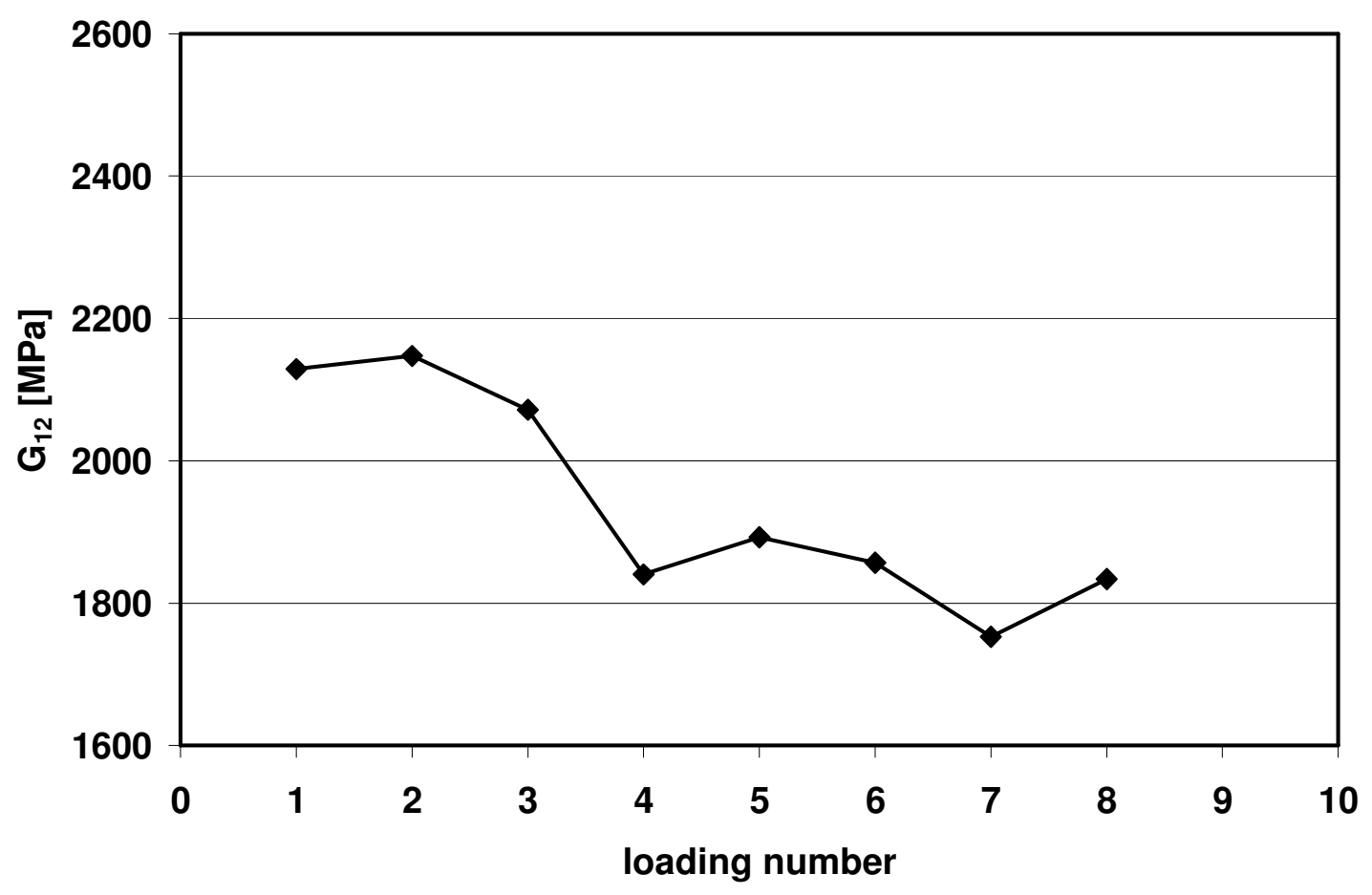

Figure 3.30 Cyclic shear slopes $\left(G_{12}\right)$ vs. loading number of specimen in Figure 3.29. Notice that with slope each reloading the slope does not necessarily decrease. 


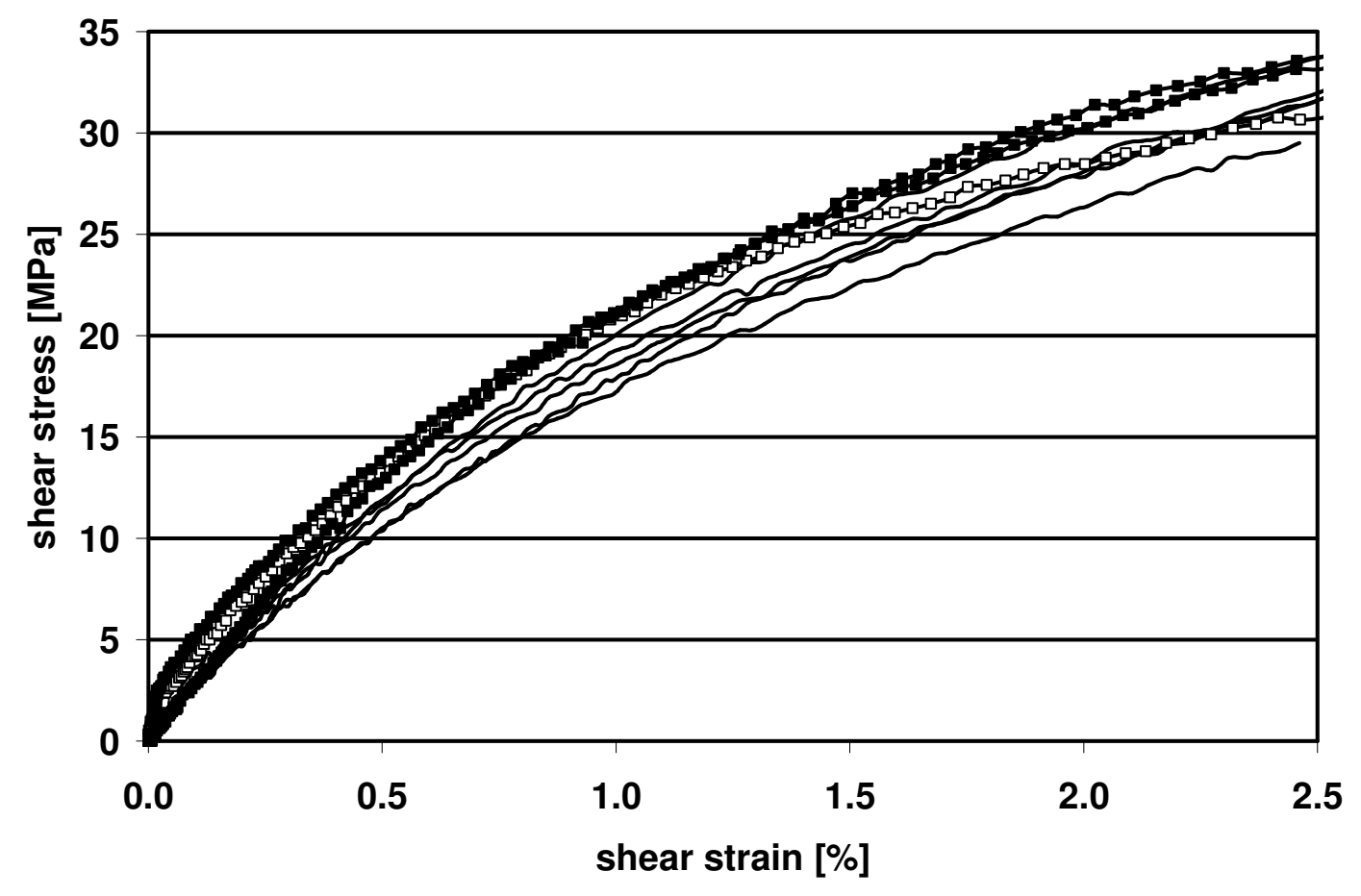

Figure 3.31 Cyclic healing shear testing, all data is shifted to the origin.

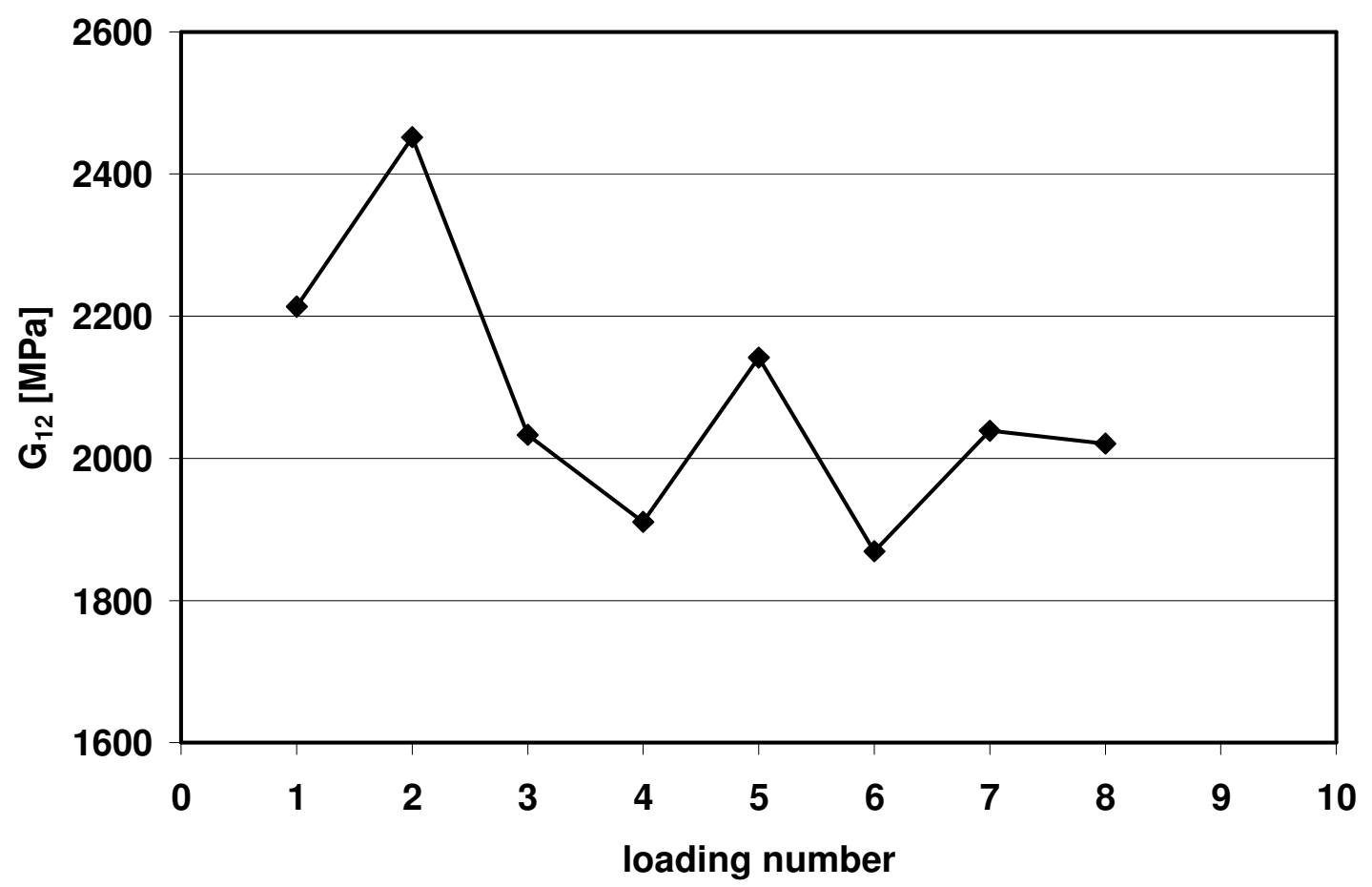

Figure 3.32 Cyclic shear slopes $\left(\mathbf{G}_{12}\right)$ vs. loading number of specimen in Figure 3.31. Notice that with slope each reloading the slope does not necessarily decrease. 


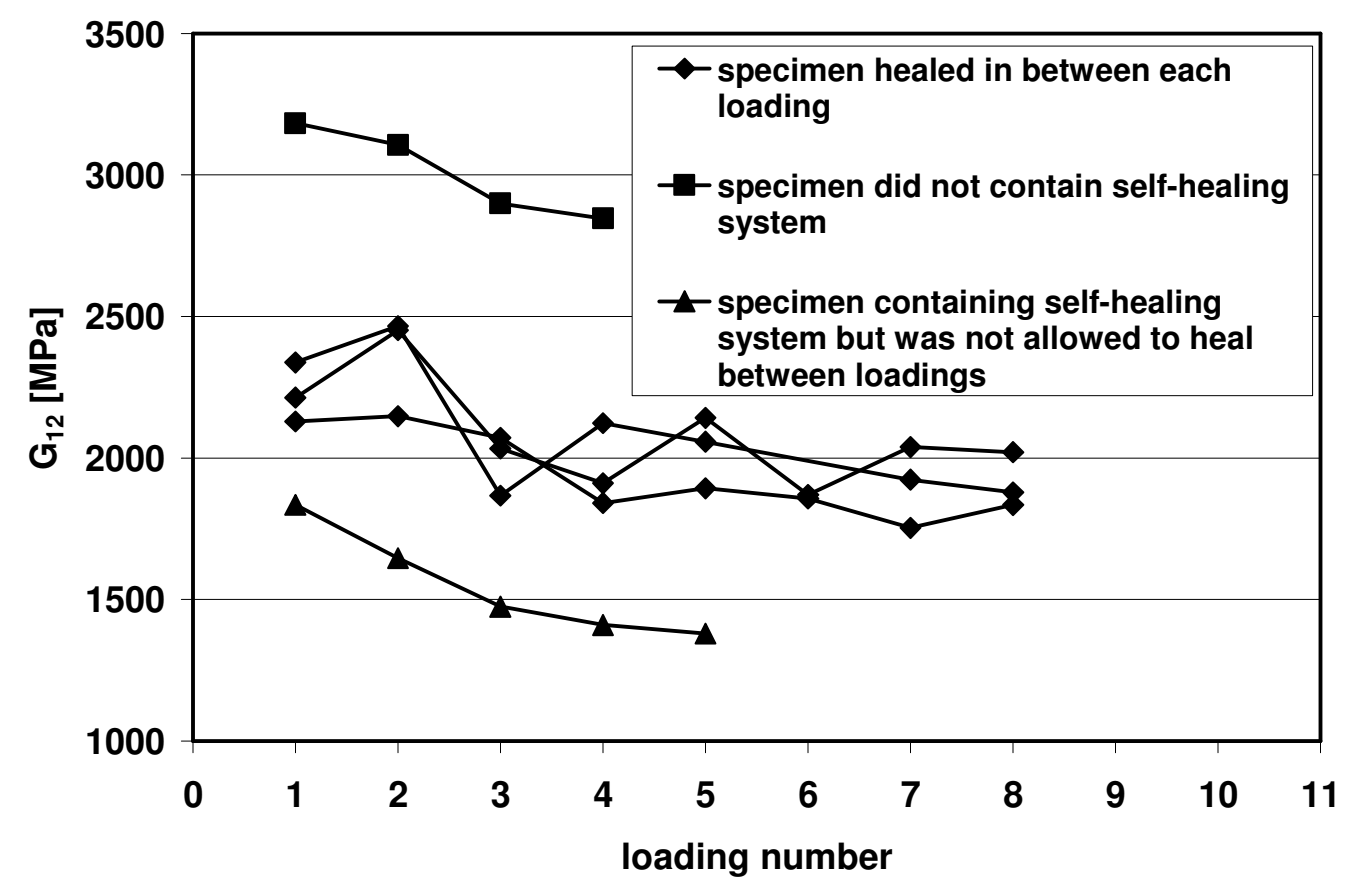

Figure 3.33 Comparison of cyclic shear slopes $\left(G_{12}\right)$ vs. loading number of specimens not containing the self-healing system, specimens containing the self-healing system that did not heal, and specimens containing the self-healing system that healed. 


\section{SIMPLIFIED DAMAGE AND HEALING MECHANICS}

\section{MODEL}

This chapter outlines the development of a simple Continuum Damage and Healing Mechanics Model, SCDHM. Lamina experimental results evidence different damage modes and evolution for longitudinal, transverse, and shear loading [1, 35]. In addition, shear loading leads to longitudinal and mostly transverse damage [14, 67, 80]. Therefore, the orientation of defects coincides with the material coordinate axes of the lamina. Since the material damages along the coordinate axes, healing must also occur along the coordinate axes. The SCDHM model extends the Continuum Damage Mechanics model (CDM) model to incorporate the healing phenomena.

This leads to the conclusion that the damage surface and the healing surface have the same shape, but different magnitudes. In this work it is proposed that a healed specimen is a damaged specimen with less damage. With the assumption that the damage and healing surfaces are the same, a single internal variable can be used to define the damage/healing surface. Figure 4.1 depicts the damage and healing surface domain and the damage threshold. At $0, \gamma$ the material is undamaged and considered a virgin material. Once the loading reaches the damage threshold, $\gamma_{o}$, the material begins to damage. Damage evolution grows and the material continues to damage until the loading stops at $\gamma^{*}$. At this point the material heals. Healing reduces the damage surface. The damage and healing cycle will continue until the amount of the healing agent is used up or the damage evolution reaches a value of 1 where the material fails. At failure the damage surface is compared to the Tsai-Wu failure criterion. 
Several types of modeling to predict behavior prior to failure have been previously reported. Ply discount methods and adjustable degradation factors are very approximate and not very accurate and have been used with limited success [46]. Micro mechanical models have been used to assemble the global response of a single ply in terms of the damaging behavior of the constituents $[87,88,90]$. Due to the large number of required parameters and the computationally intense nature of these models, they are not used to deal with laminates. Most continuum damage models require specialized tests to determine the required parameters. On the other hand, the CDHM model proposed by Barbero et al. [18] utilizes parameters that can be obtained from standard ASTM and or ISO testing.

A few assumptions were made in the development of the SCDHM model:

- A surface exists which separates the elastic domain from the damage domain. Basically a material will behave elastically without damage until the thermodynamic force reaches the damage surface, the point at which damage begins to accumulate.

- The damage principal directions coincide with the lamina's material directions.

- Healing can only occur where the material has damaged. Therefore, healing principal directions coincide with the lamina's material directions.

- The self-healing system is uniformly distributed throughout the laminate.

The constitutive relationships and evolution equations that define CDM and CDHM models, define a non-linear differential problem, which is solved through the use of a numerical algorithm. The CDM model has been validated with numerous 
comparisons of predicted damage and experimental damage $[14,16,17,18,20]$. Data from unidirectional experiments of Aramid/Epoxy and Carbon/Epoxy and data from torsion experiments of Glass/Epoxy and Carbon/Epoxy have been used in the validation of this model with great success.

The SCDHM model describes the damage and the healing phenomena through the use of internal state variables at the mesoscale. One important aspect of the SCDHM model and the self-healing concept in general is that the healing agents are uniformly distributed throughout the material. As mentioned earlier, healing can only occur when a microcrack ruptures an embedded microencapsulated healing agent. Experimental observations on polymer-matrix composites have shown that prior to failure there is a continuous distribution of microcracks in the matrix. For healing to occur the assumption that the self-healing system, both microcapsules and catalyst, must be uniformly distributed throughout the material is made. The current CDHM model available in the literature [18] has not been validated with experimental data and does not describe the type of experimental produce that is necessary to determine the material parameters.

\subsection{Damage Interpretation}

The Continuous Damage and Healing Mechanics Model (CDHM) [18] assumes that damage is orthotropic and that the principal directions of damage and healing are aligned with the material coordinates. The healing model is developed in a similar manner as the damage model and expands upon the damage model developed in [20]. A second order diagonal damage tensor is defined as

$$
D_{i j}=\sum_{i=1}^{3} d_{i} n_{i} \otimes n_{i}=\left[\begin{array}{ccc}
d_{1} & 0 & 0 \\
0 & d_{2} & 0 \\
0 & 0 & d_{3}
\end{array}\right]
$$


where $\otimes$ denotes the dyadic product of tensors [57], $\mathrm{n}_{\mathrm{i}}$ are the orthogonal principal directions, which coincide with the fiber, transverse, and thickness directions, $d_{i}$ are the eigenvalues of the damage tensor.

The dual variable of the damage tensor is the integrity tensor, $\mathbf{\Omega}=\sqrt{\mathbf{I}-\mathbf{D}}$, which represents the undamaged ratio. In indicial form it is written as

$$
\Omega_{i j}=\sqrt{\delta_{i j}-D_{i j}}=\left[\begin{array}{ccc}
\sqrt{1-d_{1}} & 0 & 0 \\
0 & \sqrt{1-d_{2}} & 0 \\
0 & 0 & \sqrt{1-d_{3}}
\end{array}\right]=\left[\begin{array}{ccc}
\Omega_{1} & 0 & 0 \\
0 & \Omega_{2} & 0 \\
0 & 0 & \Omega_{3}
\end{array}\right]
$$

The damage-healing integrity tensor is used to describe a mapping between the effective, $\overline{\mathbb{C}}$, and damaged-healed, $\mathbb{C}$, configurations by a linear operator, $f$, as $f: \mathbb{C} \rightarrow \overline{\mathbb{C}}$. The integrity tensor is always symmetric and positive, because the net area decrease must be positive definite during damage evolution.

A transformation tensor is introduced as

$$
\overline{d x_{i}}=\chi \quad \text { with } \quad i=1,2,3
$$

to express the deformation of an arbitrary segment $d x_{i}$ to $\widetilde{d x_{i}}$ between the damage-healed and effective configuration of a representative volume element.

A generic area element can then be transformed by the following equations

$$
\overline{n d S}=\frac{1}{2} \overline{d x} \times \overline{d y}=\frac{1}{2}(\chi \cdot d x) \times(\chi \cdot d y)=\operatorname{det}[\chi]\left(\chi^{-1 / 2}\right)^{T}\left(\chi^{-1 / 2}\right) n d S
$$

which is based on Nanson's Theorem [Ogden 1984] and where $(\times)$ is the vector product. The area reduction along the principal directions can be expressed in terms of the eigenvalues of the damage tensor as

$$
\left[1-d_{i}\right] n_{i} d S_{i}=\overline{n_{i}} \overline{d S_{i}}
$$


The net area change due to degradation is defined by $d_{i}$ which is the eigenvalues of the damage tensor along different planes. Using Eqs. (4.4) and (4.5) the transformation tensor in the principal reference frame can be expressed as

$$
\begin{aligned}
& \chi_{11}=\sqrt{\frac{\left(1-d_{2}\right)\left(1-d_{3}\right)}{\left(1-d_{1}\right)}} \\
& \chi_{22}=\sqrt{\frac{\left(1-d_{1}\right)\left(1-d_{3}\right)}{\left(1-d_{2}\right)}} \\
& \chi_{33}=\sqrt{\frac{\left(1-d_{1}\right)\left(1-d_{2}\right)}{\left(1-d_{3}\right)}}
\end{aligned}
$$

The derivation of the transformation tensor is described in the following steps. Looking at the terms on the right-hand side of Eq. (4.4) we have the following relations

$$
\begin{gathered}
\left(\chi^{-1 / 2}\right)^{T}=\left(\chi^{-1 / 2}\right) \\
\left(\chi^{-1 / 2}\right)\left(\chi^{-1 / 2}\right)=\left(\chi^{-1}\right)
\end{gathered}
$$

thus

$$
\begin{aligned}
& \chi_{11}^{-1}=\left(\frac{\left(1-d_{1}\right)}{\left(1-d_{2}\right)\left(1-d_{3}\right)}\right)^{1 / 2} \\
& \chi_{22}^{-1}=\left(\frac{\left(1-d_{2}\right)}{\left(1-d_{1}\right)\left(1-d_{3}\right)}\right)^{1 / 2} \\
& \chi_{33}^{-1}=\left(\frac{\left(1-d_{3}\right)}{\left(1-d_{1}\right)\left(1-d_{2}\right)}\right)^{1 / 2}
\end{aligned}
$$

and where

$$
\operatorname{det}[\chi]=\chi_{11} \chi_{22} \chi_{33}=\sqrt{\left(1-d_{1}\right)} \sqrt{\left(1-d_{2}\right)} \sqrt{\left(1-d_{3}\right)}
$$

Then substituting Eq. (4.7) through (4.10) into Eq. (4.4) and looking at the 11 component we have 


$$
\begin{gathered}
\overline{n_{1}} \overline{d S_{1}}=\left(\frac{\left(1-d_{2}\right)\left(1-d_{3}\right)}{\left(1-d_{1}\right)}\right)^{1 / 2}\left(\frac{\left(1-d_{1}\right)\left(1-d_{3}\right)}{\left(1-d_{2}\right)}\right)^{1 / 2} \\
\left(\frac{\left(1-d_{1}\right)\left(1-d_{2}\right)}{\left(1-d_{3}\right)}\right)^{1 / 2}\left(\frac{\left(1-d_{1}\right)}{\left(1-d_{2}\right)\left(1-d_{3}\right)}\right)^{1 / 2} n_{1} d S_{1}=\left(1-d_{1}\right) n_{1} d S_{1}
\end{gathered}
$$

which can be written in a general form as

$$
\overline{n_{i}} \overline{d S_{i}}=\left(1-d_{i}\right) n_{i} d S_{i}
$$

The effective stress corresponds to the first-Piola Kirchhoff tensor

$$
\bar{\sigma}=[\operatorname{det} \chi]^{-1} \chi^{-1 / 2} \sigma \chi^{-1 / 2} R=M^{-1} \sigma
$$

where $\mathrm{R}$ is a Reuter matrix defined as

$$
R=\left[\begin{array}{llllll}
1 & 0 & 0 & 0 & 0 & 0 \\
0 & 1 & 0 & 0 & 0 & 0 \\
0 & 0 & 1 & 0 & 0 & 0 \\
0 & 0 & 0 & 2 & 0 & 0 \\
0 & 0 & 0 & 0 & 2 & 0 \\
0 & 0 & 0 & 0 & 0 & 2
\end{array}\right]
$$

and $\mathrm{M}$ is a symmetric fourth order tensor, called the effective damage tensor. The effective damage/healing tensor is used to relate the stress and strain in the effective and damage/healing configurations and is defined as

$$
M=\operatorname{det}[\chi] \chi^{-1 / 2} \chi^{-1 / 2} R^{-1}
$$

where 


$$
\begin{gathered}
M_{11}=M_{1111}=\operatorname{det}[\chi] \chi_{11}^{-1 / 2} \chi_{11}^{-1 / 2} R_{11}^{-1}=\left(1-d_{1}\right) \\
M_{22}=M_{2222}=\left(1-d_{2}\right) \\
M_{33}=M_{3333}=\left(1-d_{3}\right) \\
M_{44}=M_{2323}=\operatorname{det}[\chi] \chi_{22}^{-1 / 2} \chi_{33}^{-1 / 2} R_{44}^{-1}=\frac{\sqrt{\left(1-d_{2}\right)} \sqrt{\left(1-d_{3}\right)}}{2} \\
M_{55}=\frac{\sqrt{\left(1-d_{1}\right)} \sqrt{\left(1-d_{3}\right)}}{2} \\
M_{66}=\frac{\sqrt{\left(1-d_{1}\right)} \sqrt{\left(1-d_{2}\right)}}{2}
\end{gathered}
$$

Using the damage integrity tensor, the effective damage tensor can be rewritten in the following form

$$
M=\operatorname{diag}\left\{\left(\Omega_{1}\right)^{2} ;\left(\Omega_{2}\right)^{2} ;\left(\Omega_{3}\right)^{2} ; \frac{\Omega_{2} \Omega_{3}}{2} ; \frac{\Omega_{1} \Omega_{3}}{2} ; \frac{\Omega_{1} \Omega_{2}}{2}\right\}
$$

The effective damage tensor is used as a linear operator used to relate the stress and stain in the effective and damage configurations. These relations are defined as

$$
\begin{aligned}
& \bar{\sigma}_{i j}=M_{i j k l}^{-1} \sigma_{k l} \\
& \bar{\varepsilon}_{i j}^{e}=M_{i j k l} \varepsilon_{k l}^{e}
\end{aligned}
$$

where an over-bar indicates that the quantity is evaluated in the effective configuration and superscript $e$ denotes quantities in the elastic domain. Using the above relations, the effective stress and strain components can be written in contracted notation as the following expressions 


$$
\begin{gathered}
\bar{\sigma}_{11}=\frac{\chi_{11} \sigma_{11}}{\operatorname{det}(\chi)}=\frac{\sigma_{11}}{\left(1-d_{1}\right)}=\sigma_{11}\left(\Omega_{1}\right)^{-2} \\
\bar{\sigma}_{22}=\frac{\chi_{22} \sigma_{22}}{\operatorname{det}(\chi)}=\frac{\sigma_{22}}{\left(1-d_{2}\right)}=\sigma_{22}\left(\Omega_{2}\right)^{-2} \\
\bar{\sigma}_{33}=\frac{\chi_{33} \sigma_{33}}{\operatorname{det}(\chi)}=\frac{\sigma_{33}}{\left(1-d_{3}\right)}=\sigma_{33}\left(\Omega_{3}\right)^{-2} \\
\bar{\sigma}_{12}=\frac{\left[\chi_{11}\right]^{1 / 2} \sigma_{12}\left[\chi_{22}\right]^{1 / 2}}{\operatorname{det}(\chi)}=\frac{\sigma_{12}}{\sqrt{1-d_{1}} \sqrt{1-d_{2}}}=\sigma_{12}\left(\Omega_{1} \Omega_{2}\right)^{-1} \\
\bar{\sigma}_{13}=\frac{\left[\chi_{11}\right]^{1 / 2} \sigma_{13}\left[\chi_{33}\right]^{1 / 2}}{\operatorname{det}(\chi)}=\frac{\sigma_{12}}{\sqrt{1-d_{1}} \sqrt{1-d_{3}}}=\sigma_{13}\left(\Omega_{1} \Omega_{3}\right)^{-1} \\
\bar{\sigma}_{23}=\frac{\left[\chi_{22}\right]^{1 / 2} \sigma_{23}\left[\chi_{33}\right]^{1 / 2}}{\operatorname{det}(\chi)}=\frac{\sigma_{23}}{\sqrt{1-d_{2}} \sqrt{1-d_{3}}}=\sigma_{23}\left(\Omega_{2} \Omega_{3}\right)^{-1}
\end{gathered}
$$

and

$$
\begin{gathered}
\bar{\varepsilon}_{11}=\varepsilon_{11}\left(1-d_{1}\right)=\varepsilon_{11}\left(\Omega_{1}\right)^{2} \\
\bar{\varepsilon}_{22}=\varepsilon_{22}\left(1-d_{2}\right)=\varepsilon_{22}\left(\Omega_{2}\right)^{2} \\
\bar{\varepsilon}_{33}=\varepsilon_{33}\left(1-d_{3}\right)=\varepsilon_{33}\left(\Omega_{3}\right)^{2} \\
\bar{\varepsilon}_{12}=\varepsilon_{12} \sqrt{1-d_{1}} \sqrt{1-d_{2}}=\varepsilon_{12} \Omega_{1} \Omega_{2} \\
\bar{\varepsilon}_{13}=\varepsilon_{13} \sqrt{1-d_{1}} \sqrt{1-d_{3}}=\varepsilon_{13} \Omega_{1} \Omega_{3} \\
\bar{\varepsilon}_{23}=\varepsilon_{23} \sqrt{1-d_{2}} \sqrt{1-d_{3}}=\varepsilon_{23} \Omega_{2} \Omega_{3}
\end{gathered}
$$

By the energy equivalence hypothesis $[26,27,61]$, it is possible to define the constitutive equation in the effective configuration, $\overline{\mathbb{C}}$, as

$$
\begin{gathered}
\bar{\sigma}_{i j}=\bar{C}_{i j k l} \bar{\varepsilon}_{k l}^{e} \\
\overline{\mathcal{\varepsilon}}_{i j}^{e}=\bar{C}_{i j k l}^{-1} \bar{\sigma}_{k l}=\bar{S}_{i j k l} \bar{\sigma}_{k l}
\end{gathered}
$$

where the fourth order tensors, $\mathbf{C}$ and $\mathbf{S}$, denote the secant stiffness tensor and compliance tensor respectively. The constitutive equation in the damaged configuration, $\mathbb{C}$, is obtained by substituting Eqs. (4.21) into Eqs. (4.18) 


$$
\begin{gathered}
\sigma_{i j}=M_{i j k l} \bar{\sigma}_{k l}=M_{i j k l} \bar{C}_{k l r s} \bar{\varepsilon}_{r s}^{e}, \\
\sigma_{i j}=M_{i j k l} \bar{C}_{k l r s} M_{r s t u} \varepsilon_{t u}^{e}, \\
\sigma_{i j}=C_{i j k l} \varepsilon_{k l}^{e} \\
\varepsilon_{i j}^{e}=M_{i j k l}^{-1} \bar{\varepsilon}_{k l}^{e}=M_{i j k l}^{-1} \bar{S}_{k l r s} \bar{\sigma}_{r s}, \\
\varepsilon_{i j}^{e}=M_{i j k l}^{-1} \bar{S}_{k l r s} M_{i j k l}^{-1} \sigma_{t u}, \\
\varepsilon_{i j}^{e}=S_{i j k l} \sigma_{k l}
\end{gathered}
$$

The relationship between the damaged and effective configurations can be written as

$$
\begin{aligned}
C_{i j k l} & =M_{i j k l} \bar{C}_{k l r s} M_{r s t u} \\
S_{i j k l} & =M_{i j k l}^{-1} \bar{S}_{k l r s} M_{r s t u}^{-1}
\end{aligned}
$$

Given that the tensor $\mathbf{M}$ is symmetric, the secant stiffness tensor and compliance tensor, $\mathbf{C}$ and $\mathbf{S}$, as a result, are also symmetric. Using Eqs. (4.23), the stiffness tensor written in terms of the effective stiffness tensor is

$$
\begin{aligned}
C_{i j} & =\left[\begin{array}{ccc}
\bar{C}_{11}\left(\Omega_{1}\right)^{4} & \bar{C}_{12}\left(\Omega_{1} \Omega_{2}\right)^{2} & \bar{C}_{13}\left(\Omega_{1} \Omega_{3}\right)^{2} \\
\bar{C}_{12}\left(\Omega_{1} \Omega_{2}\right)^{2} & \bar{C}_{22}\left(\Omega_{2}\right)^{4} & \bar{C}_{23}\left(\Omega_{2} \Omega_{3}\right)^{2} \\
\bar{C}_{13}\left(\Omega_{1} \Omega_{3}\right)^{2} & \bar{C}_{23}\left(\Omega_{2} \Omega_{3}\right)^{2} & \bar{C}_{33}\left(\Omega_{3}\right)^{4}
\end{array}\right], i, j=1,3 \\
C_{i j} & =\left[\begin{array}{ccc}
\bar{C}_{44}\left(\Omega_{2} \Omega_{3}\right)^{2} & 0 & 0 \\
0 & \bar{C}_{55}\left(\Omega_{1} \Omega_{3}\right)^{2} & 0 \\
0 & 0 & \bar{C}_{66}\left(\Omega_{1} \Omega_{2}\right)^{2}
\end{array}\right], i, j=4,6
\end{aligned}
$$

where $\bar{C}_{44}=2 \cdot \bar{G}_{23}, \bar{C}_{55}=2 \cdot \bar{G}_{13}$ and $\bar{C}_{66}=2 \cdot \bar{G}_{12}$. The Voigt contracted notation for fourth-order elasticity tensors is used here: $C_{\alpha \beta}$ replaces $C_{i j k l}$ where $\alpha, \beta$ take the values 1 , $2,3,4,5,6$, corresponding to the indicial pairs $11,22,33,23,13$ and 12 , respectively.

\subsubsection{Thermodynamic Formulations}

Healing is not instantaneous. Unlike the model described in [19], time is needed to allow the healing mechanism to cure. The self-healing system used in this research for 
example takes $48 \mathrm{hrs}$. to sufficiently cure [23]. Therefore, during damage the healing tensor is assumed to be zero, $H=0$. Therefore, the CDM part of the SCDHM model is derived the same way as the CDM in [20].

\subsubsection{Unrecoverable Deformation, and Damage}

The damage surface is defined using two fourth order tensors, $\mathrm{J}$ and $\mathrm{B}$, and is written as

$$
g^{d}=\left(\hat{Y}_{i j}^{N} \cdot J_{i j h k} \hat{Y}_{h k}^{N}\right)^{1 / 2}+\left(Y_{i j}^{S} \cdot B_{i j h k} Y_{h k}^{S}\right)^{1 / 2}-\left(\gamma(\delta)+\gamma_{0}\right)
$$

The diagonal fourth order positive defined tensors, $\mathbf{J}$ and $\mathbf{B}$ are determined by available data on a single composite lamina as discussed in the Model Identification section of [20], which is explained in detail in Chapter 6. The $\mathbf{Y}^{\mathrm{N}}$ and $\mathbf{Y}^{\mathrm{S}}$ are, respectively, the thermodynamic forces from normal strains and the thermodynamic forces from shear strains. The thermodynamic forces from normal strains can be defined as

$$
Y_{i j}^{N}=\frac{1}{2} \varepsilon_{k l}^{e} \delta_{k l} \delta_{m n} \frac{\partial C_{m n p q}}{\partial D_{i j}} \delta_{p q} \delta_{r s} \varepsilon_{r s}^{e}
$$

The addition of $\mathbf{Y}^{\mathrm{N}}$ and $\mathbf{Y}^{\mathrm{S}}$ is the thermodynamic force tensor, $\mathbf{Y}$, defined as

$$
Y_{i j}=-\frac{\partial \psi}{\partial D_{i j}}=-\frac{1}{2}\left(\varepsilon_{k l}-\varepsilon_{k l}^{p}\right) \frac{\partial C_{k l p q}}{\partial D_{i j}}\left(\varepsilon_{p q}-\varepsilon_{p q}^{p}\right)=-\frac{1}{2} \varepsilon_{k l}^{e} \frac{\partial C_{k l p q}}{\partial D_{i j}} \varepsilon_{p q}^{e}
$$

The thermodynamic forces from shear strains can be written as

$$
Y_{i j}^{S}=Y_{i j}-Y_{i j}^{N}
$$

$\hat{\mathbf{Y}}^{\mathrm{N}}$ is the mapped thermodynamic force from normal strain and is defined as

$$
\hat{Y}_{i j}^{N}=\hat{A}_{i j k l} Y_{k l}^{N}
$$


The tensor $\hat{\mathbf{A}}$ is a diagonal transformation tensor introduced in order to preserve convexity of the damage threshold surface in stress-strain and thermodynamics force spaces. Referring to the principal directions this tensor can be written as

$$
\hat{A}_{i j k l}=\delta_{i m} \delta_{j m} \delta_{k m} \delta_{l m}+\left(A_{m}-1\right) \delta_{i m} \delta_{j m} \delta_{k m} \delta_{l m} r_{m}
$$

where

$$
r_{m}=\frac{1}{2}\left(1-\frac{\varepsilon_{m}}{\left|\varepsilon_{m}\right|}\right)=\left\{\begin{array}{lll}
0 & \text { if } & \sigma_{m}>0 \\
1 & \text { if } & \sigma_{m}<0
\end{array}\right.
$$

with $m=1,2,3$, the values of $\varepsilon_{m}$ are the values of the normal strain in the principal material directions and the three values $A_{m}$ of the vector $\mathbf{A}$ represents the relation between damage thresholds between uniaxial compression and uniaxial extension also in the principal material directions.

The second order tensor of the conjugate thermodynamic forces associated to the damage and healing variables takes the following shape

$$
Y_{i j}=\left[\begin{array}{rrr}
Y_{1} & 0 & 0 \\
0 & Y_{2} & 0 \\
0 & 0 & Y_{3}
\end{array}\right]
$$

The explicit expressions for the thermodynamic forces written in terms of effective elastic strain are found as

$$
\begin{gathered}
Y_{1}=\frac{1}{\left(\Omega_{1}\right)^{2}}\left(\bar{C}_{11} \bar{\varepsilon}_{11}^{e} \bar{\varepsilon}_{11}^{e}+\bar{C}_{12} \bar{\varepsilon}_{11}^{e} \bar{\varepsilon}_{22}^{e}+\bar{C}_{13} \bar{\varepsilon}_{11}^{e} \bar{\varepsilon}_{33}^{e}+\bar{C}_{55} \bar{\varepsilon}_{13}^{e} \bar{\varepsilon}_{13}^{e}+\bar{C}_{66} \bar{\varepsilon}_{12}^{e} \bar{\varepsilon}_{12}^{e}\right) \\
Y_{2}=\frac{1}{\left(\Omega_{2}\right)^{2}}\left(\bar{C}_{22} \bar{\varepsilon}_{22}^{e} \bar{\varepsilon}_{22}^{e}+\bar{C}_{12} \bar{\varepsilon}_{11}^{e} \bar{\varepsilon}_{22}^{e}+\bar{C}_{23} \bar{\varepsilon}_{22}^{e} \bar{\varepsilon}_{33}^{e}+\bar{C}_{44} \bar{\varepsilon}_{23}^{e} \bar{\varepsilon}_{23}^{e}+\bar{C}_{66} \bar{\varepsilon}_{12}^{e} \bar{\varepsilon}_{12}^{e}\right) \\
Y_{3}=\frac{1}{\left(\Omega_{3}\right)^{2}}\left(\bar{C}_{33} \bar{\varepsilon}_{33}^{e} \bar{\varepsilon}_{33}^{e}+\bar{C}_{13} \bar{\varepsilon}_{11}^{e} \bar{\varepsilon}_{33}^{e}+\bar{C}_{23} \bar{\varepsilon}_{22}^{e} \bar{\varepsilon}_{33}^{e}+\bar{C}_{44} \bar{\varepsilon}_{23}^{e} \bar{\varepsilon}_{23}^{e}+\bar{C}_{55} \bar{\varepsilon}_{13}^{e} \bar{\varepsilon}_{13}^{e}\right)
\end{gathered}
$$


Which can be written in terms of engineering stress as

$$
\begin{aligned}
& Y_{1}=\frac{1}{\left(\Omega_{1}\right)^{2}}\left[\frac{\bar{S}_{11} \sigma_{11}^{2}}{\left(\Omega_{1}\right)^{4}}+\frac{\bar{S}_{12} \sigma_{11} \sigma_{22}}{\left(\Omega_{1} \Omega_{2}\right)^{2}}+\frac{\bar{S}_{13} \sigma_{11} \sigma_{33}}{\left(\Omega_{1} \Omega_{3}\right)^{2}}+\frac{\bar{S}_{55} \sigma_{13}^{2}}{\left(\Omega_{1} \Omega_{3}\right)^{2}}+\frac{\bar{S}_{66} \sigma_{12}^{2}}{\left(\Omega_{1} \Omega_{2}\right)^{2}}\right] \\
& Y_{2}=\frac{1}{\left(\Omega_{2}\right)^{2}}\left[\frac{\bar{S}_{22} \sigma_{22}^{2}}{\left(\Omega_{2}\right)^{4}}+\frac{\bar{S}_{12} \sigma_{11} \sigma_{22}}{\left(\Omega_{1} \Omega_{2}\right)^{2}}+\frac{\bar{S}_{23} \sigma_{22} \sigma_{33}}{\left(\Omega_{2} \Omega_{3}\right)^{2}}+\frac{\bar{S}_{44} \sigma_{13}^{2}}{\left(\Omega_{2} \Omega_{3}\right)^{2}}+\frac{\bar{S}_{66} \sigma_{12}^{2}}{\left(\Omega_{1} \Omega_{2}\right)^{2}}\right] \\
& Y_{3}=\frac{1}{\left(\Omega_{3}\right)^{2}}\left[\frac{\bar{S}_{33} \sigma_{33}^{2}}{\left(\Omega_{1}\right)^{4}}+\frac{\bar{S}_{13} \sigma_{11} \sigma_{33}}{\left(\Omega_{1} \Omega_{3}\right)^{2}}+\frac{\bar{S}_{23} \sigma_{22} \sigma_{33}}{\left(\Omega_{2} \Omega_{3}\right)^{2}}+\frac{\bar{S}_{44} \sigma_{23}^{2}}{\left(\Omega_{2} \Omega_{3}\right)^{2}}+\frac{\bar{S}_{55} \sigma_{13}^{2}}{\left(\Omega_{1} \Omega_{3}\right)^{2}}\right]
\end{aligned}
$$

On the other hand, the unrecoverable-deformation (yield) evolution is modeled by classical plasticity formulation [54], as in [17]. An associate flow rule is assumed in the effective stress space, coupling plasticity and damage effects. The unrecoverabledeformation surface is a function of the thermodynamic forces in the effective configuration $(\overline{\boldsymbol{\sigma}}, R)$. Therefore, the unrecoverable-deformation yield surface, which accounts for thickness terms, is

$$
g^{p}=\sqrt{f_{i j} \bar{\sigma}_{i} \bar{\sigma}_{j}+f_{i} \bar{\sigma}_{i}}-\left(R(p)+R_{o}\right)
$$

where $(i=1,2 \ldots 6), R_{o}$ is the unrecoverable-deformation energy threshold and $R$ is defined as

$$
R(p)=-\frac{\partial \psi}{\partial p}=c_{1}^{p}\left[\exp \left(\frac{p}{c_{2}^{p}}\right)-1\right]
$$

A tri-dimensional Tsai-Wu criterion shape is chosen for Eq. (4.35)because of its ability to represent different behavior among the different load paths in stress space. The coefficients $f_{i}$ and $f_{i j}$ assume the following form 


$$
\begin{gathered}
f_{1}=\frac{1}{F_{1 t}}-\frac{1}{F_{1 c}} ; \quad f_{2}=\frac{1}{F_{2 t}}-\frac{1}{F_{2 c}} ; \quad f_{3}=\frac{1}{F_{3 t}}-\frac{1}{F_{3 c}} ; \\
f_{11}=\frac{1}{F_{1 t} F_{1 c}} ; \quad f_{22}=\frac{1}{F_{2 t} F_{2 c}} ; \quad f_{33}=\frac{1}{F_{3 t} F_{3 c}} ; \\
f_{44}=\frac{1}{F_{4}^{2}} ; \quad f_{55}=\frac{1}{F_{5}^{2}} ; \quad f_{66}=\frac{1}{F_{6}^{2}} ; \\
f_{23} \cong-\frac{0.5}{\left(F_{2 t} F_{2 c} F_{3 t} F_{3 c}\right)} ; \quad f_{13} \cong-\frac{0.5}{\left(F_{1 t} F_{1 c} F_{3 t} F_{3 c}\right)} ; \quad f_{12} \cong-\frac{0.5}{\left(F_{1 t} F_{1 c} F_{2 t} F_{2 c}\right)}
\end{gathered}
$$

The parameters $F_{i t}, F_{i c}$, and $F_{i}$ are the strength values in tension, compression, in-plane, and out-of-plane shear for a single composite lamina. These values are tabulated in literature, or they can be easily obtained following standardized test methods $[7,8,10]$.

\subsection{Healing Interpretation}

Unlike previous healing models [19], the SCDHM model uses the same thermodynamic space for damage and healing. Just like the damage tensor, the principal directions of the healing tensor are thought to coincide with the material coordinates. Since it is thought that damage occurs along the principal directions, healing can only occur where there is damage. Healing is represented by a diagonal second order healing tensor, $\mathbf{H}$, defined as

$$
H_{i j}=\sum_{i=1}^{3} h_{i} n_{i} \otimes n_{i}=\left[\begin{array}{ccc}
h_{1} & 0 & 0 \\
0 & h_{2} & 0 \\
0 & 0 & h_{3}
\end{array}\right]
$$

where $\otimes$ denotes the outer product, or the dyadic product, of tensors, $n_{i}$ are the orthogonal principal directions, which coincide with the fiber, transverse, and thickness directions, and $h_{i}$ are the eigenvalues of the $\mathbf{H}$ tensor, which represents the healing ratio along the $\mathrm{n}_{\mathrm{i}}$ directions. 
The healing tensor, $\mathbf{H}$, is a function of the damage tensor and the healing efficiency, $\eta$.

$$
H=f(D, \eta)
$$

In this work, the healing tensor is approximated as

$$
H=\eta D
$$

The healing efficiency is calculated from shear stress - shear strain data and is defined using the method outlined below. First, the '66' component in Eq. (4.24) can be written as

$$
G_{12}^{d}=G_{12}^{o}\left(1-d_{1}\right)\left(1-d_{2}\right)
$$

where $G_{12}^{d}$ is the shear modulus of the specimen during unloading (Figure 4.2) and $G_{12}^{o}$ is the shear modulus of the undamaged (virgin) specimen (Figure 4.2). Healing counteracts the effects of damage; therefore, the following relationship between the damage and healing tensor is made

$$
D^{h}=D-H
$$

where $D^{h}$ is the damage tensor after healing occurs, $D$ is the damage tensor, and $H$ is the healing tensor. Using the assumption that a healed specimen is still a damaged specimen and that no healing occurs in the ' 1 ' direction, $h_{1}=0$, Eq. (4.41) can be rewritten as

$$
\begin{aligned}
& G_{12}^{d}=G_{12}^{o}\left(1-d_{2}\right) \\
& G_{12}^{h}=G_{12}^{o}\left(1-d_{2}^{h}\right)
\end{aligned}
$$

where $G_{12}^{h}$ is the shear modulus of the specimen after it has healed (Figure 4.2). 


$$
d_{2}=-\frac{G_{12}^{d}}{G_{12}^{o}}+1
$$

Next, substitute the '2' component of Eq. (4.42) into Eq. (4.44) the following equation is obtained

$$
G_{12}^{h}=G_{12}^{o}\left(1-d_{2}+h_{2}\right)
$$

Then by using the relationship in Eq. (4.40) for the ' 2 ' direction

$$
G_{12}^{h}=G_{12}^{o}\left(1-d_{2}(1-\eta)\right)
$$

To calculate the healing efficiency substitute Eq. (4.45) into Eq. (4.47) which yields

$$
\eta=\frac{G_{12}^{h}-G_{12}^{d}}{G_{12}^{o}-G_{12}^{d}}
$$

The unloading shear modulus, $G_{12}^{d}$, from the Iosipescu shear test is difficult to measure and the data has too much scatter. Therefore, the healing efficiency was solved by calculating $G_{12}^{d}$ using Eq. (4.45) and is written as

$$
\eta=\frac{\frac{G_{12}^{h}}{G_{12}^{o}}-1+d_{2}}{d_{2}}
$$

During unloading of the Iosipescu specimens measurement of the unrecoverable strain is difficult and the data has too much scatter. It is believed that this is caused by either one of several factors or a combination of them. One error in this measurement could be caused by the slack in the fixture's test machine adaptor (Figure 4.3). Slack occurs from the spring and ball bearing used to align the upper and lower loading planes of the fixture. When the specimen is unloaded the MTS testing machine is returned to zero load and zero displacement but the strain gages do not return to zero. The strain gages themselves might have some type of residual strain reading. 
Once an equation to calculate the healing efficiency is established, a relationship between damage and the healing efficiency is then determined. The healing coefficients $c_{1}^{h}, c_{2}^{h}, c_{3}^{h}$, and $c_{4}^{h}$ define the healing efficiency curve, or healing potential, are defined using the following equation

$$
\eta_{i}=\left(c_{1}^{h}\right)^{4} d_{i}+\left(c_{2}^{h}\right)^{3} d_{i}+\left(c_{3}^{h}\right)^{2} d_{i}+c_{4}^{h}
$$

where $d_{i}$ is the damage in the ' $\mathrm{i}$ ' direction and $\eta_{i}$ is the healing efficiency in the ' $\mathrm{i}$ ' direction. The identification of the healing parameters, $c_{1}^{h}, \ldots$, is defined in Section 5.5 Identification of Healing Parameters.

The damage hardening parameter, $\delta$, can be thought of as a rope. As each strand in the rope breaks the rope as whole becomes weaker, or softer. The same principal applies to a composite material. When a part of the composite (either the fiber or the matrix) breaks, weakening the material, the material becomes softer. But a self-healing composite can counteract this and become harder again. In order to calculate the amount of hardening regained due to healing, $\mu$, the total damage that can be healed, $d_{\text {heal }}$, is calculated using the following equation

$$
d_{\text {heal }}=d_{2}+d_{3}
$$

Since the self-healing system can only heal damage in the matrix the damage that can be healed in the fiber direction is zero, $d_{1}=0$. Next, the percentage of the total damage that can be healed in each direction is calculated using the following equations

$$
\begin{gathered}
d_{\text {heal }}=\frac{d_{2}}{d_{\text {heal }}} \\
d_{\text {heal }}=\frac{d_{3}}{d_{\text {heal }}}
\end{gathered}
$$


Using the healing efficiency the amount of hardening recovered from healing in each direction can be calculated using the following equation

$$
\begin{aligned}
& \mu_{2}=\eta_{2} \delta \\
& \mu_{3}=\eta_{3} \delta
\end{aligned}
$$

Then by using Eqs. (4.52) and (4.53) the overall hardening recovered from healing is calculated using the following equation

$$
\mu=\mu_{2} d_{\text {heal }}+\mu_{3} d_{\text {heal }}
$$

$d_{\text {heal }}$ and $d_{\text {heal }}$ can be thought of as weight factors. They take into account that the amount of healing that occurs in each direction is different. The amount of hardening recovered is dependent upon the amount of healing that occurs in each direction. If the damage and healing phenomena are dominant in one direction then that direction's healing efficiency will control how much hardening is recovered. Lastly, the damage hardening parameter is updated using the following equation

$$
\delta=\delta-\mu
$$

\subsection{Numerical Implementation}

The numerical implementation of the model described in the previous chapter is outlined next. A displacement-based finite element formulation is used. The geometry is discretized by three-dimensional composite elements and the material non-linearity is tracked at each Gauss integration point. In order to integrate the rate equations, an algorithm for coupled damage and unrecoverable-strain is developed in [20]. A returnmapping algorithm is used for the numerical integration of the damage-healing constitutive equations. The damage and unrecoverable-strain threshold functions are written by means of a linearized procedure between two iterations from constitutive 
equation algorithm integration, as described in the following steps. The procedure is identical as the on proposed in [20] except the addition of step 8.

1. Displacement increment for step " $\mathrm{n}$ " $\Delta u_{i}^{n}$ is obtained from an equilibrium equation written in agreement with finite element formulation. The incremental and updated strains are easily obtained using the following equations [20]

$$
\begin{gathered}
\Delta \varepsilon_{i j}^{n}=\frac{1}{2}\left(\Delta u_{i, j}^{n}+\Delta u_{j, i}^{n}\right) \\
\varepsilon_{i j}^{n}=\varepsilon_{i j}^{n-1}+\Delta \varepsilon_{i j}^{n}
\end{gathered}
$$

2. The state variables from the previous step "n-1" are obtained by starting the returnmapping algorithm with damage and unrecoverable-strains, setting the predictor $\mathrm{k}=0$ [20]. Therefore

$$
\begin{gathered}
\left(D_{i j}\right)_{0}^{n}=\left(D_{i j}\right)^{n-1} ; \quad\left(H_{i j}\right)_{0}^{n}=\left(H_{i j}\right)^{n-1} ; \quad(\delta)_{0}^{n}=(\delta)^{n-1} \\
\left(\varepsilon_{i j}^{p}\right)_{0}^{n}=\left(\varepsilon_{i j}^{p}\right)^{n-1} ; \quad(p)_{0}^{n}=(p)^{n-1}
\end{gathered}
$$

3. The thermodynamic force, stress tensor, and hardening evaluation are then evaluated at this point [20]

$$
\left(Y_{i j}\right)_{k}^{n} ; \quad\left(\hat{Y}_{i j}^{N}\right)_{k}^{n} ; \quad\left(Y_{i j}^{S}\right)_{k}^{n} ; \quad(\gamma)_{k}^{n} ; \quad\left(\bar{\sigma}_{i j}\right)_{k}^{n} ; \quad(R)_{k}^{n}
$$

4. The damage and unrecoverable-strain threshold are then evaluated at this point [20]

$$
\begin{gathered}
\left(G^{d}\right)_{k}=g^{d}\left(\left(\hat{Y}_{i j}^{N}\right)_{k}^{n},\left(Y_{i j}^{S}\right)_{k}^{n},(\gamma(\delta))_{k}^{n}, \gamma_{o}\right) \\
\left(G^{p}\right)_{k}=g^{p}\left(\left(\bar{\sigma}_{i j}\right)_{k}^{n},(R(p))_{k}^{n}, R_{o}\right)
\end{gathered}
$$

Four different conditions define all possible cases:

a) If $\left(G^{d}\right)_{k} \leq 0$ and $\left(G^{p}\right) \leq 0$ elastic behavior. Therefore, go to step 8 .

b) If $\left(G^{d}\right)_{k}>0$ and $\left(G^{p}\right) \leq 0$ only damage evolution, $\Delta \lambda_{k}^{p}=0$ and $\Delta \lambda_{k}^{d} \neq 0$. 
c) If $\left(G^{d}\right)_{k} \leq 0$ and $\left(G^{p}\right)>0$ only unrecoverable-strain evolution, $\Delta \lambda_{k}^{p} \neq 0$ and $\Delta \lambda_{k}^{d}=0$.

d) If $\left(G^{d}\right)_{k}>0$ and $\left(G^{p}\right)>0$ damage and unrecoverable-strain evolution. $\Delta \lambda_{k}^{p} \neq 0$ and $\Delta \lambda_{k}^{d} \neq 0$.

5. Starting from the iteration $k=k+1$, from linearization to the first order of the linear equation system in Eq. () the following equations can be written [20]

$$
\begin{aligned}
& \left(G^{d}\right)_{k}-\left(G^{d}\right)_{k-1}=\frac{\partial g^{d}}{\partial Y_{i j}}\left[\frac{\partial Y_{i j}}{\partial \varepsilon^{p}{ }_{k l}} M_{k l m n}^{-1} \Delta \lambda_{k}^{p} \frac{\partial g^{p}}{\partial \bar{\sigma}_{m n}}+\frac{\partial Y_{i j}}{\partial D_{k l}} \Delta \lambda_{k}^{d} \frac{\partial g^{d}}{\partial Y_{k l}}\right]+\frac{\partial g^{d}}{\partial \gamma} \frac{\partial \gamma}{\partial \delta} \Delta \lambda_{k}^{d} \frac{\partial g^{d}}{\partial \gamma} \\
& \left(G^{p}\right)_{k}-\left(G^{p}\right)_{k-1}=\frac{\partial g^{p}}{\partial{\overline{\sigma_{i j}}}_{k}}\left[\frac{\partial \bar{\sigma}_{i j}}{\partial \varepsilon^{p}{ }_{k l}} M_{k l m n}^{-1} \Delta \lambda_{k}^{p} \frac{\partial g^{p}}{\partial \bar{\sigma}_{m n}}+\frac{\partial \bar{\sigma}_{i j}}{\partial D_{k l}} \Delta \lambda_{k}^{d} \frac{\partial g^{d}}{\partial Y_{k l}}\right]+\frac{\partial g^{p}}{\partial R} \frac{\partial R}{\partial p} \Delta \lambda_{k}^{p} \frac{\partial g^{p}}{\partial R}
\end{aligned}
$$

By setting $\left(G^{d}\right)_{k}=0$ and $\left(G^{p}\right)_{k}=0$ the following linear system is obtained

$$
\begin{aligned}
& -\left(G^{d}\right)_{k-1}=\left[\frac{\partial g^{d}}{\partial Y_{i j}} \frac{\partial Y_{i j}}{\partial D_{k l}} \frac{\partial g^{d}}{\partial Y_{k l}}+\frac{\partial g^{d}}{\partial \gamma} \frac{\partial \gamma}{\partial \delta} \frac{\partial g^{d}}{\partial \gamma}\right] \Delta \lambda_{k}^{d}+\left[\frac{\partial g^{d}}{\partial Y_{i j}} \frac{\partial Y_{i j}}{\partial \mathcal{E}^{p}{ }_{k l}} M_{k l m n}^{-1} \frac{\partial g^{p}}{\partial \bar{\sigma}_{m n}}\right] \Delta \lambda_{k}^{p} \\
& -\left(G^{p}\right)_{k-1}=\left[\frac{\partial g^{p}}{\partial \bar{\sigma}_{i j}} \frac{\partial \bar{\sigma}_{i j}}{\partial D_{k l}} \frac{\partial g^{d}}{\partial Y_{k l}}\right] \Delta \lambda_{k}^{d}+\left[\frac{\partial g^{p}}{\partial \bar{\sigma}_{i j}} \frac{\partial \bar{\sigma}_{i j}}{\partial \varepsilon_{k l}^{p}} M_{k l m n}^{-1} \frac{\partial g^{p}}{\partial \bar{\sigma}_{m n}}+\frac{\partial g^{p}}{\partial R} \frac{\partial R}{\partial p} \frac{\partial g^{p}}{\partial R}\right] \Delta \lambda_{k}^{p}
\end{aligned}
$$

The previous equation reduces to

$$
\begin{aligned}
& -\left(G^{d}\right)_{k-1}=a^{11} \Delta \lambda_{k}^{d}+a^{21} \Delta \lambda_{k}^{p} \\
& -\left(G^{p}\right)_{k-1}=a^{12} \Delta \lambda_{k}^{d}+a^{22} \Delta \lambda_{k}^{p}
\end{aligned}
$$

from which both consistency factors $\Delta \lambda_{k}^{d}$ and $\Delta \lambda_{k}^{p}$ are computed.

a. If damage evolution exists, then the damage consistency factor, $\Delta \lambda_{k}^{d}$, is

$$
\left(G^{d}\right)_{k}=0 \Rightarrow \Delta \lambda_{k}^{d}=\frac{-\left(a^{22}\right)_{k-1} \cdot\left(G^{d}\right)_{k-1}+\left(a^{12}\right)_{k-1} \cdot\left(G^{p}\right)_{k-1}}{\left(a^{11}\right)_{k-1} \cdot\left(a^{22}\right)_{k-1}-\left(a^{12}\right)_{k-1} \cdot\left(a^{21}\right)_{k-1}}
$$

b. If unrecoverable-strain evolution exists, then the unrecoverable-strain consistency factor, $\Delta \lambda_{k}^{p}$, is 


$$
\left(G^{p}\right)_{k}=0 \Rightarrow \Delta \lambda_{k}^{p}=\frac{-\left(a^{11}\right)_{k-1} \cdot\left(G^{p}\right)_{k-1}+\left(a^{21}\right)_{k-1} \cdot\left(G^{d}\right)_{k-1}}{\left(a^{11}\right)_{k-1} \cdot\left(a^{22}\right)_{k-1}-\left(a^{12}\right)_{k-1} \cdot\left(a^{21}\right)_{k-1}}
$$

6. Increment and update the state variables according to the consistency factors $\Delta \lambda_{k}^{d}$ and $\Delta \lambda_{k}^{p}$ using the following equations [20]

$$
\begin{gathered}
\left(D_{i j}\right)_{k}^{n}=\left(D_{i j}\right)_{k-1}^{n}+\Delta \lambda_{k}^{d}\left(\frac{\partial f^{d}}{\partial Y_{i j}}\right)_{k-1} \\
(\delta)_{k}^{n}=(\delta)_{k-1}^{n}+\Delta \lambda_{k}^{d}\left(\frac{\partial f^{d}}{\partial \gamma}\right)_{k-1}=(\delta)_{k-1}^{n}-\Delta \lambda_{k}^{d} \\
\left(\varepsilon_{i j}^{p}\right)_{k}^{n}=\left(\varepsilon_{i j}^{p}\right)_{k-1}^{n}+\Delta \lambda_{k}^{p}\left(\frac{\partial g^{p}}{\partial \bar{\sigma}_{i j}}\right)_{k-1} \\
(p)_{k}^{n}=(p)_{k-1}^{n}+\Delta \lambda_{k}^{p}\left(\frac{\partial g^{p}}{\partial R}\right)_{k-1}=(p)_{k-1}^{n}-\Delta \lambda_{k}^{p}
\end{gathered}
$$

7. End of the linearized process. Go to step 3.

8. Check if healing occurs at the current substep. A state variable is used to tell the program if healing occurs or not. If the variable equals 0 healing does not occur, if the variable equals 1 healing occurs.

a. If healing does not occur go to step 9 .

b. If healing does occur.

i. Calculate the percent recovered in the ' 2 ' and ' 3 ' directions.

$$
\eta_{i}=\left(c_{1}^{h}\right)^{4} d_{i}+\left(c_{2}^{h}\right)^{3} d_{i}+\left(c_{3}^{h}\right)^{2} d_{i}+c_{4}^{h} d_{i}+c_{5}^{h}
$$

ii. Calculate the healing tensor.

$$
\begin{gathered}
h_{1}=0 \\
h_{2}=\eta_{2} d_{2} \\
h_{3}=\eta_{3} d_{3}
\end{gathered}
$$

iii. Update damage tensor. 


$$
\left(D_{i j}\right)_{k}^{n}=\left(D_{i j}\right)_{k}^{n}-\left(H_{i j}\right)_{k}^{n}
$$

9. Set up the stress, damage and unrecoverable strain variables for the next load increment in each integration point using the following equations [20]

$$
\begin{gathered}
\left(D_{i j}\right)^{n}=\left(D_{i j}\right)_{k}^{n} ; \quad(\delta)^{n}=(\delta)_{k}^{n} \\
\left(\varepsilon_{i j}^{p}\right)^{n}=\left(\varepsilon_{i j}^{p}\right)_{k}^{n} ; \quad(p)^{n}=(p)_{k}^{n} \\
\left(\sigma_{i j}\right)^{n}=\left(M_{i j k l}\right)^{n} \bar{C}_{k l r s}\left(M_{r s t u}\right)^{n}\left[\left(\varepsilon_{t u}\right)^{n}-\left(\varepsilon_{t u}^{p}\right)^{n}\right]
\end{gathered}
$$

10. Compute the tangent constitutive tensor [20]

$$
\begin{aligned}
\left(C_{i j r s}^{e p d h}\right)^{n}=\left(M_{i j k l}\right)^{n} & \bar{C}_{k l m n}\left(M_{m n p q}\right)^{n}\left(I_{p q r s}-\left(M_{p q t u}^{-1}\right)^{n}\left(L_{r s}^{p}\right)^{n}\left(\frac{\partial g^{p}}{\partial \bar{\sigma}_{t u}}\right)^{n}\right) \\
& +\left(\frac{\partial \sigma_{i j}}{\partial D_{p q}}\right)^{n}\left(L_{r s}^{d}\right)^{n}\left(\frac{\partial f^{d}}{\partial Y_{p q}}\right)^{n}
\end{aligned}
$$

11. End of the constitutive equation integration algorithm 


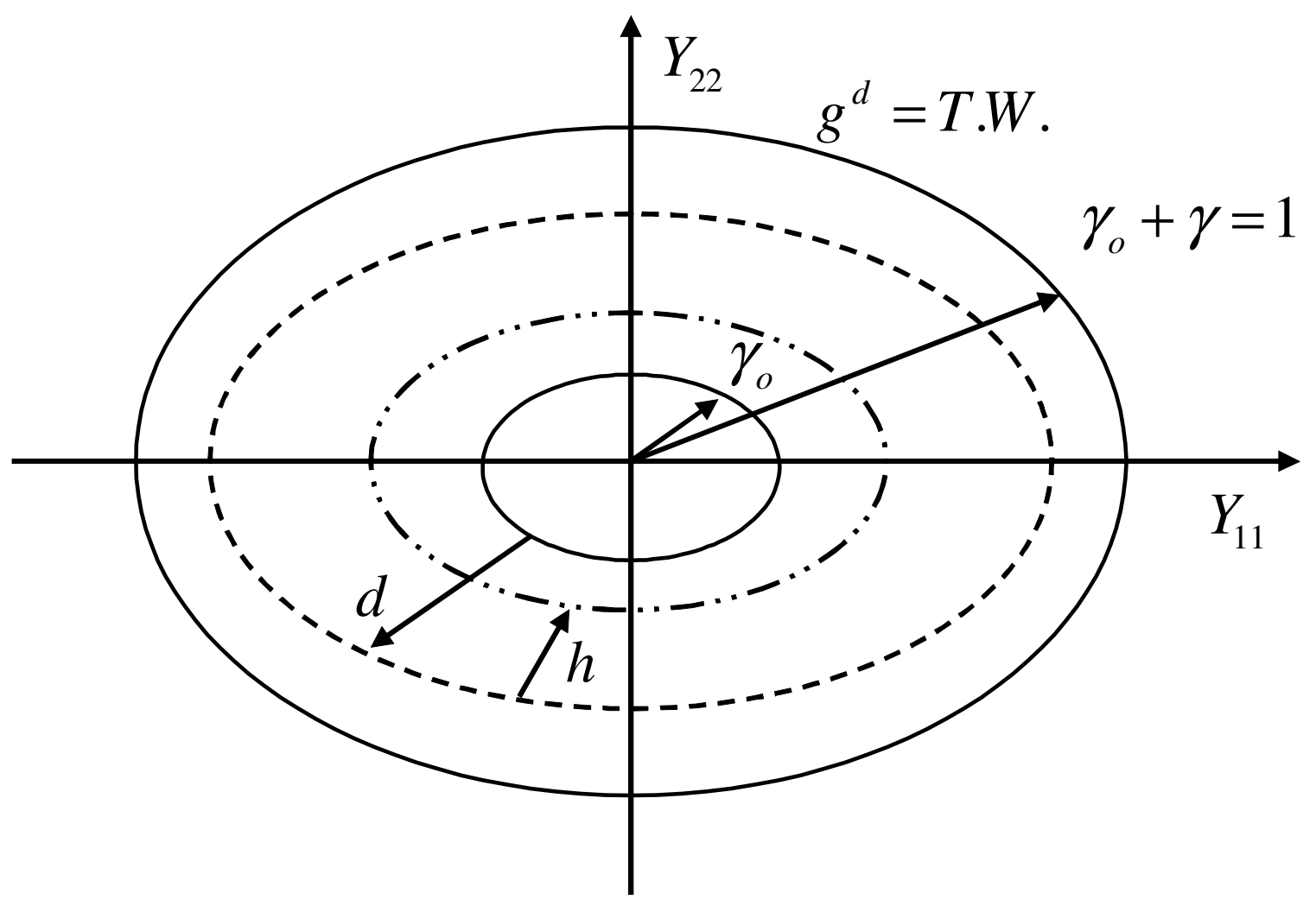

Figure 4.1 Diagram of the damage/healing domain and damage threshold.

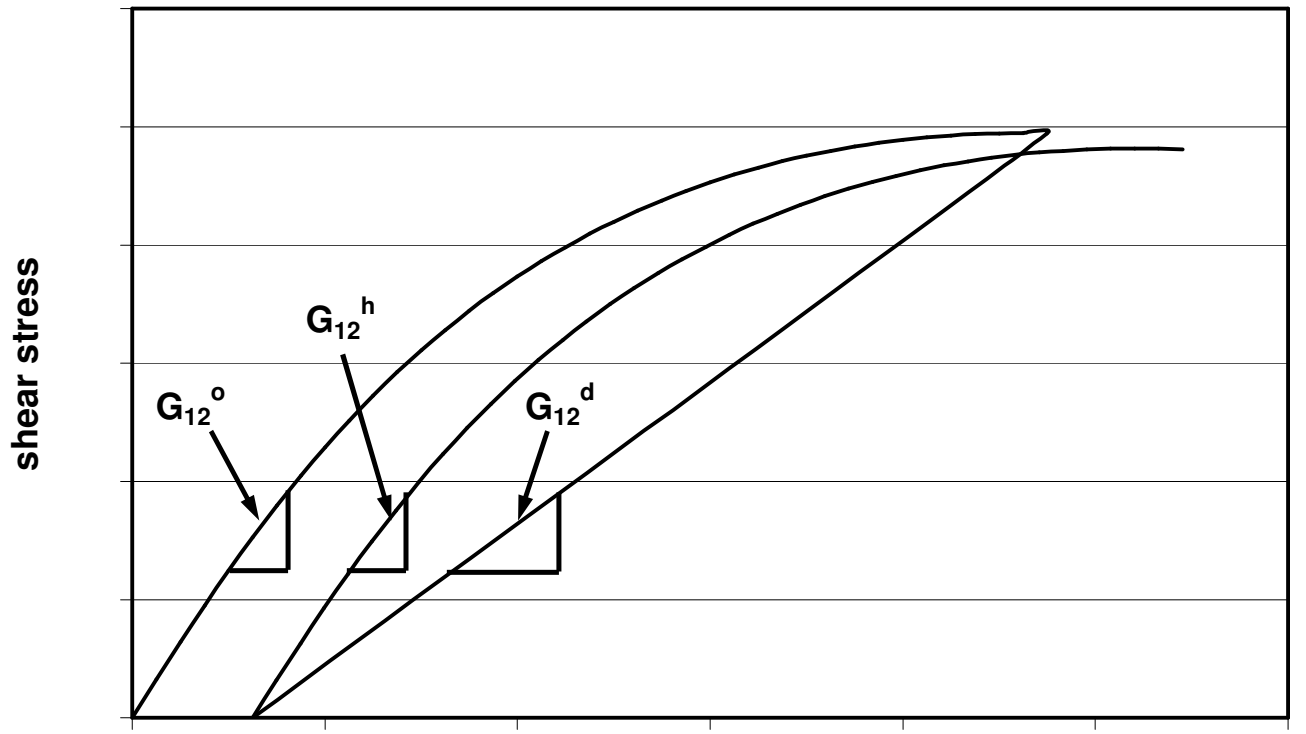

shear strain

Figure 4.2 Shear loading cycle for a self-healing composite material depicting the initial shear modulus, damaged shear modulus, and healed shear moduls. 


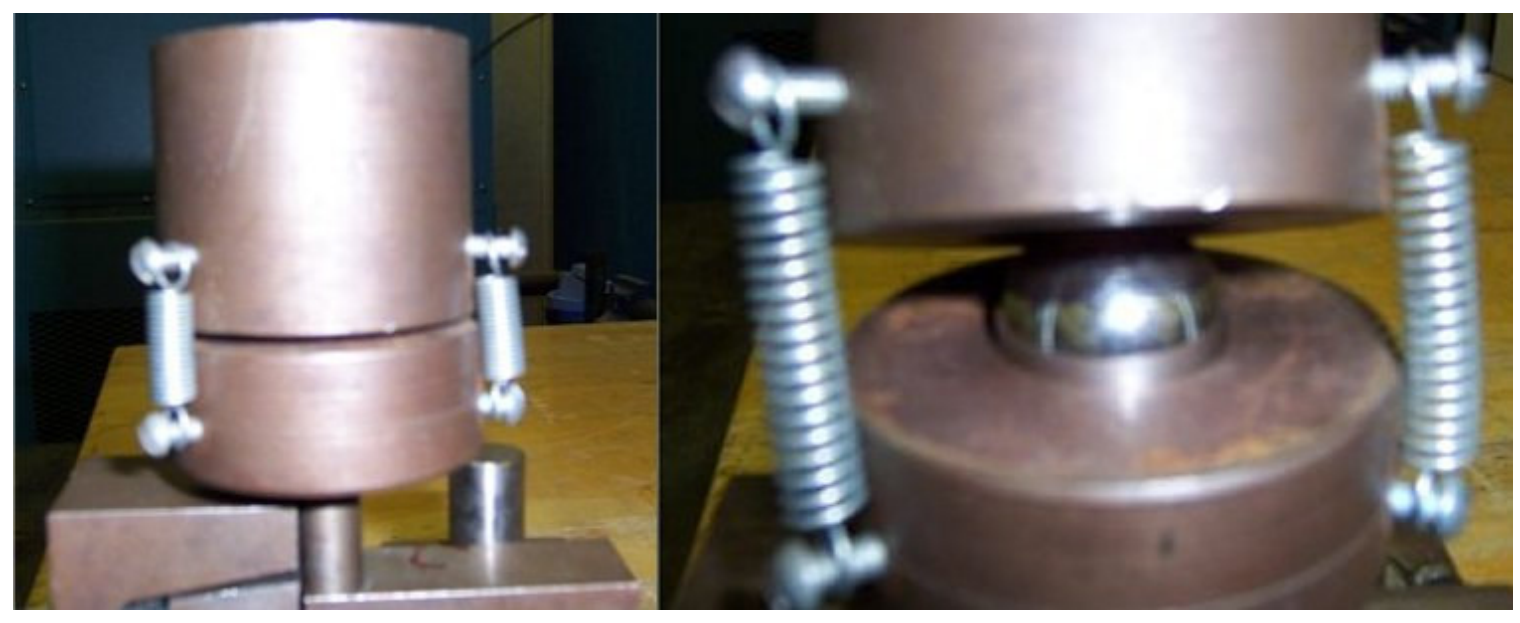

Figure 4.3 Iosipescu test adaptor. 


\section{MODEL IDENTIFICATION}

\subsection{Determination of the Critical Damage Parameters}

Barbero et al. [16] discuss how to determine the critical values of the damage parameters introduced in Eq. (4.1). The critical values are the amount of damage present when the material fails. In other words, the critical values, $d_{1 t}, d_{2 t}, d_{3 t}$, etc. are essentially the most damage that a material can endure before it fails.

Critical values are estimated from statistical models of the failure process for each type of loading. Examining a lamina that is loaded along the fiber direction, it can be assumed that the fibers carry the load and no damage is expected in the matrix during loading. Therefore, the ultimate tensile strength of the composite lamina can be accurately predicted by computing the strength of a bundle of fibers [16]. The critical damage $\mathrm{d}_{1 \mathrm{t}}$ for longitudinal tensile loading can be computed as the area fraction of broken fibers in the lamina, if a Weibull distribution is assumed for the strength of the fibers [69] and no significant initial fiber damage is assumed. The critical damage is a function of the Weibull shape modulus $m$

$$
d_{1 t}=1-\exp \left(\frac{1}{m}\right)
$$

Fiber micro-buckling appears to be the predominant mode of damage when a fiber-reinforced lamina is compressed [12, 86, 91]. Fiber misalignment lowers the buckling load from that of a perfect system. For each misalignment angle, $\alpha$, the composite area-fraction with buckled fibers $d(\alpha)$, corresponding to fibers with a misalignment angle greater than $\alpha$, can be taken as a measure of damage [16]. If the assumption that the fibers have no post-buckling strength, then the applied stress is 
redistributed onto the remaining unbuckled fibers, which in turn will carry a higher effective stress. The applied stress, which is lower than the effective stress by the factor (1-d), has a maximum, which corresponds to the compressive strength of the composite. It is possible to compute the critical damage $d_{1 c}$ for longitudinal compressive loading as

$$
d_{1 c}=1-\operatorname{erf}\left(\frac{\alpha_{c r}}{\Lambda \sqrt{2}}\right)
$$

where erf is the error function, $\Lambda$ is the standard deviation of the actual Gaussian distribution of fiber misalignment, and $\alpha_{\mathrm{cr}}$ is the critical misalignment angle at failure.

Transverse tension is assumed to be controlled by brittle fracture of the matrix and the brittle loose bundle model is assumed. A simple flat distribution can be assumed for the probability of matrix-link failure

$$
p(f)=\left(\frac{1}{\sigma_{o}}\right)
$$

in terms of the strength of the strongest matrix-link $\sigma_{o}$. The maximum stress in the bundle of matrix links turns out to be

$$
\sigma_{c}=\frac{\sigma_{o}}{4}
$$

so that the percentage of links which are broken prior to failure is [38]

$$
d_{2 t}=d_{3 t}=0.5
$$

\subsection{Damage Surface Identification}

Damage does not occur until the thermodynamic forces reach the damage surface, Eq. (4.25). For an undamaged material $\gamma_{o}=0$, while at failure $\gamma^{*}+\gamma_{o}=1$ and the damage surface is described by the Tsai-Wu failure criterion. A comparison of the 
damage surface and the Tsai-Wu failure criterion leads to a linear system of equations that allows one to determine the internal material parameters $\mathbf{J}, \mathbf{B}$, and $\mathbf{A}$, Eqs. (4.25) and (4.29).

The internal material properties are dependant upon the overall material properties which are determined experimentally using standard testing methods. The internal material parameters are calculated using the series of equations discussed in the proceeding sections. A more detailed description the determination of the damage and plasticity internal material parameters can be found in Barbero and Mayugo [20]. The required material properties are

- The stiffness values: $\mathrm{E}_{1}, \mathrm{E}_{2}=\mathrm{E}_{3}, \mathrm{G}_{12}=\mathrm{G}_{13}, \mathrm{G}_{23}, \mathrm{v}_{12}=\mathrm{v}_{13}$, and $\mathrm{v}_{23}$

- The strength values: $F_{1 t}, F_{1 c}, F_{2 c}=F_{3 c}, F_{2 t}=F_{3 t}, F_{4}$, and $F_{5}=F_{6}$

- The critical damage values: $\mathrm{d}_{1 \mathrm{t}}, \mathrm{d}_{1 \mathrm{c}}$, and $\mathrm{d}_{2 \mathrm{t}}=\mathrm{d}_{3 \mathrm{t}}$

- The damaged shear moduli at imminent failure: $G_{12}^{*}$ and $G_{13}^{*}$

- The in-plane shear elastic limit: $F_{6 E p}$

- The undamaged shear limit: $F_{6 E d}$

- The unrecoverable strain: $\varepsilon_{12}^{p}$ as a function of applied $\sigma_{6}$

- The healing efficiency parameters: $c_{1}^{h}, c_{2}^{h}, c_{3}^{h}$, and $c_{4}^{h}$

The damage domain is defined by fourth order tensors $\mathbf{J}$ and $\mathbf{B}$, and the vector $\mathbf{A}$. The tensor $\mathbf{J}$ and the vector $\mathbf{A}$ are directly related to material properties obtained by tensile and compression tests of unidirectional lamina in the principal material directions. The damge surface function, $g^{d}$, for longitudinal uniaxial tension and compression of an unidirectional lamina, $\left(\sigma_{11} \neq 0, \sigma_{22}=\sigma_{33}=\sigma_{12}=\sigma_{13}=\sigma_{23}=0\right)$, is written as [20] 


$$
\begin{gathered}
\sqrt{J_{11}} \frac{\overline{S_{11}}}{\Omega_{1 t}^{6}} F_{1 t}^{2}=\left(\gamma^{*}+\gamma_{o}\right)=1 \\
\sqrt{J_{11}} A_{1} \frac{\overline{S_{11}}}{\Omega_{1 c}^{6}} F_{1 c}^{2}=\left(\gamma^{*}+\gamma_{o}\right)=1
\end{gathered}
$$

For a unidirectional lamina under transverse tensile and compression stress $\left(\sigma_{22} \neq 0, \sigma_{11}=\sigma_{33}=\sigma_{12}=\sigma_{13}=\sigma_{23}=0\right)$ the damage surface function is written as [20]

$$
\begin{gathered}
\sqrt{J_{22}} \frac{\overline{S_{22}}}{\Omega_{2 t}^{6}} F_{2 t}^{2}=\left(\gamma^{*}+\gamma_{o}\right)=1 \\
\sqrt{J_{22}} A_{2} \frac{\overline{S_{22}}}{\Omega_{2 c}^{6}} F_{2 c}^{2}=\left(\gamma^{*}+\gamma_{o}\right)=1
\end{gathered}
$$

and in the thickness direction $\left(\sigma_{33} \neq 0, \sigma_{11}=\sigma_{22}=\sigma_{12}=\sigma_{13}=\sigma_{23}=0\right)$ the damage surface function is written as [20]

$$
\begin{gathered}
\sqrt{J_{33}} \frac{\overline{S_{33}}}{\Omega_{3 t}^{6}} F_{3 t}^{2}=\left(\gamma^{*}+\gamma_{o}\right)=1 \\
\sqrt{J_{33}} A_{\frac{1}{3}} \frac{\overline{S_{33}}}{\Omega_{3 c}^{6}} F_{3 c}^{2}=\left(\gamma^{*}+\gamma_{o}\right)=1
\end{gathered}
$$

where $F_{1 t}, F_{1 c}, F_{2 t}, F_{2 c}, F_{3 t}$, and $F_{3 c}$ are the strength values and $\overline{S_{11}}, \overline{S_{22}}$, and $\overline{S_{33}}$ are the coefficients in the undamaged compliance tensor. The quantities $\Omega_{1 t}, \Omega_{1 c}, \Omega_{2 t}, \Omega_{2 c}$, $\Omega_{3 t}$, and $\Omega_{3 c}$ are the critical values of the integrity tensor at failure, as discussed in [17]. The system of Eqs. (5.6), (5.7), and (5.8) can be solved to obtain the unknown coefficients $J_{11}, J_{22}, J_{33}, A_{1}, A_{2}$, and $A_{3}$

$$
\begin{aligned}
& J_{11}=\frac{\Omega_{1 t}^{6}}{{\overline{S_{11}}}^{2}} \frac{1}{F_{1 t}^{4}}, \quad J_{22}=\frac{\Omega_{2 t}^{6}}{{\overline{S_{22}}}^{2}} \frac{1}{F_{2 t}^{4}}, \quad J_{33}=\frac{\Omega_{3 t}^{6}}{{\overline{S_{33}}}^{2}} \frac{1}{F_{3 t}^{4}} \\
& A_{1}=\frac{\Omega_{1 c}^{6}}{\Omega_{1 t}^{6}} \frac{F_{1 t}^{2}}{F_{1 c}^{2}}, \quad A_{2}=\frac{\Omega_{2 c}^{6}}{\Omega_{2 t}^{6}} \frac{F_{2 t}^{2}}{F_{2 c}^{2}}, \quad A_{3}=\frac{\Omega_{3 c}^{6}}{\Omega_{3 t}^{6}} \frac{F_{3 t}^{2}}{F_{3 c}^{2}}
\end{aligned}
$$

where all values of $\mathbf{J}$ and $\mathbf{A}$ are positive. 
The shear stress-strain coefficients, $B_{11}, B_{22}$ and $B_{33}$, are directly related to available material properties obtained by shear test of unidirectional lamina. Considering in-plane shear loading $\left(\sigma_{12} \neq 0, \sigma_{11}=\sigma_{22}=\sigma_{33}=\sigma_{13}=\sigma_{23}=0\right)$ and longitudinalthickness loading $\left(\sigma_{13} \neq 0, \sigma_{11}=\sigma_{22}=\sigma_{33}=\sigma_{12}=\sigma_{23}=0\right)$, and substituting in Eq. (4.34), the damage surface function, $g^{d}$, reduces to [20]

$$
\begin{aligned}
& \sqrt{\frac{B_{11}}{\Omega_{1 s}^{4}}+\frac{B_{22}}{\Omega_{2 s}^{4}}} \frac{\overline{S_{66}}}{\Omega_{1 s}^{2} \Omega_{2 s}^{2}} F_{6}^{2}=\left(\gamma^{*}+\gamma_{o}\right)=1 \\
& \sqrt{\frac{B_{11}}{\Omega_{1 s}^{4}}+\frac{B_{33}}{\Omega_{3 s}^{4}}} \frac{\overline{S_{55}}}{\Omega_{1 s}^{2} \Omega_{3 s}^{2}} F_{5}^{2}=\left(\gamma^{*}+\gamma_{o}\right)=1
\end{aligned}
$$

where $\Omega_{1 \mathrm{~s}}, \Omega_{2 \mathrm{~s}}, \Omega_{3 \mathrm{~s}}$, are the critical values of the integrity tensor at shear failure in the longitudinal, transverse, and thickness directions, respectively, and $\overline{S_{55}}$ and $\overline{S_{66}}$ are coefficients in the undamaged compliance tensor.

The parameter $B_{11}$ represents the effect of fiber damage on the damage surface caused by shear loading. But shear loading has a negligible effect on fiber damage [20]. The other two parameters $B_{22}$ and $B_{33}$, control the effect of matrix damage on the damage surface caused by shear loading and are much more significant than $B_{11}$. Therefore, it is reasonable to assume

$$
B_{11} \approx 0
$$

Therefore, the components of the $\mathbf{B}$ tensor are

$$
\begin{gathered}
B_{11}=0 \\
B_{22}=\frac{r_{12}^{S}\left(k_{12}^{S}\right)^{3}}{{\overline{S_{66}}}^{2}} \frac{1}{F_{6}^{4}} \approx\left(\frac{G_{12}^{*}}{G_{12}}\right) \frac{1}{{\overline{S_{66}}}^{2}} \frac{1}{F_{6}^{4}} \\
B_{33}=\frac{r_{13}^{S}\left(k_{13}^{S}\right)^{3}}{{\overline{S_{55}}}^{2}} \frac{1}{F_{5}^{4}} \approx\left(\frac{G_{13}^{*}}{G_{13}}\right) \frac{1}{{\overline{S_{55}}}^{2}} \frac{1}{F_{5}^{4}}
\end{gathered}
$$


where all values of $B_{i i}$ are non-negative and $k_{i}^{S}$ and $r_{i}^{S}$ are auxiliary parameters which are further discussed in [20].

\subsection{Unrecoverable-strain Surface Identification}

The unrecoverable-strain surface is defined by Eq. (4.35). A tri-dimensional Tsai$\mathrm{Wu}$ criterion shape is chosen for Eq. (4.35) because of its ability to represent different behavior among the different load paths in stress space [20]. The coefficients $f_{i}$ and $f_{i j}$ assume the following form

$$
\begin{gathered}
f_{1}=\frac{1}{\bar{F}_{1 t}}-\frac{1}{\bar{F}_{1 c}} ; \quad f_{2}=\frac{1}{\bar{F}_{2 t}}-\frac{1}{\bar{F}_{2 c}} ; \quad f_{3}=\frac{1}{\bar{F}_{3 t}}-\frac{1}{\bar{F}_{3 c}} ; \\
f_{11}=\frac{1}{\bar{F}_{1 t} \bar{F}_{1 c}} ; \quad f_{22}=\frac{1}{\bar{F}_{2 t} \bar{F}_{2 c}} ; \quad f_{33}=\frac{1}{\bar{F}_{3 t} \bar{F}_{3 c}} ; \\
f_{44}=\frac{1}{\bar{F}_{4}^{2}} ; \quad f_{55}=\frac{1}{\bar{F}_{5}^{2}} ; \quad f_{66}=\frac{1}{\bar{F}_{6}^{2}} ; \\
f_{23} \cong-\frac{1}{2} \frac{1}{\left(\bar{F}_{2 t} \bar{F}_{2 c} \bar{F}_{3 t} \bar{F}_{3 c}\right)^{1 / 2}} ; \\
f_{13} \cong-\frac{1}{2} \frac{1}{\left(\bar{F}_{1 t} \bar{F}_{1 c} \bar{F}_{3 t} \bar{F}_{3 c}\right)^{1 / 2}} ; \\
f_{12} \cong-\frac{1}{2} \frac{0.5}{\left(\bar{F}_{1 t} \bar{F}_{1 c} \bar{F}_{2 t} \bar{F}_{2 c}\right)^{1 / 2}}
\end{gathered}
$$

The parameters $\bar{F}_{i c}, \bar{F}_{i t}$, and $\bar{F}_{i}$ are the effective strength values and they are defined as:

$$
\begin{gathered}
\bar{F}_{1 t}=\frac{F_{1 t}}{\Omega_{1 t}} ; \quad \bar{F}_{2 t}=\frac{F_{2 t}}{\Omega_{2 t}} ; \quad \bar{F}_{3 t}=\frac{F_{3 t}}{\Omega_{3 t}} ; \\
\bar{F}_{1 c}=\frac{F_{1 c}}{\Omega_{1 c}} ; \quad \bar{F}_{2 c}=\frac{F_{2 c}}{\Omega_{2 c}} ; \quad \bar{F}_{3 c}=\frac{F_{3 c}}{\Omega_{3 c}} ; \\
\bar{F}_{4}=\frac{F_{4}}{\sqrt{k_{12}^{S} k_{13}^{S}}} ; \quad \bar{F}_{5}=\frac{F}{\sqrt{k_{13}^{S}}} ; \quad \bar{F}_{6}=\frac{F_{6}}{\sqrt{k_{12}^{S}}}
\end{gathered}
$$


where the parameters $F_{i t}, F_{i c}$, and $F_{i}$ are the strength values in tension, compression, inplane and out-of-plane shear for a single composite lamina. The parameters $k_{i}^{S}$ (with $i=12,13)$ are the ratios between the damaged $G_{i}^{*}$ and the undamaged $\bar{G}_{i}$ defined in [20].

\subsection{Identification of the Hardening Laws}

The unrecoverable-strain evolution and the damage evolution are defined by two independent isotropic hardening laws. The in-plane shear stress-strain curve of a single composite lamina is used to identify these hardening evolution functions

$$
\begin{aligned}
& R(p)=-\frac{\partial \psi}{\partial p}=c_{1}^{p}\left[\exp \left(\frac{p}{c_{2}^{p}}\right)-1\right] \\
& \gamma(\delta)=-\frac{\partial \psi}{\partial \delta}=c_{1}^{d}\left[\exp \left(\frac{\delta}{c_{2}^{d}}\right)-1\right]
\end{aligned}
$$

A representative in-plane stress-strain shear behavior is shown in Figure 5.1 [20].

In order to advance the hardening parameters identification it is needful to define the in-plane shear elastic limit, named $F_{6 E p}$, and the undamaged shear limit, $F_{6 E d}$, i.e. the higher shear stress values with not significant unrecoverable-strain and not significant damage, respectively. Experimental evidence [35] suggest that in fiber reinforced composites unrecoverable-strains appear after damage. Therefore, it is assumed that

$$
F_{6 E d} \leq F_{6 E p}
$$

The damage hardening parameters, $c_{1}^{d}, c_{2}^{d}$, and $\gamma_{\mathrm{o}}$, describe the evolution of the damage surface during a load increment. The damage hardening parameters, $c_{1}^{d}$ and $c_{2}^{d}$, define a damage hardening exponential function $\gamma(\delta)$ and the damage threshold, $\gamma_{\mathrm{o}}$, defines the initial size of the damage surface. 
Evolution of the unrecoverable-strain yield surface is also defined assuming an isotropic hardening law. It is also expressed by an exponential function, $R(p)$, which is dependent upon two constants, $c_{1}^{p}$ and $c_{2}^{p}$, and an initial threshold value $R_{\mathrm{o}}$.

When the material is under pure in-plane shear stress lower than the value $\sigma_{6 E p}$ the material is undamaged, hence $\delta=0$ and $\Omega_{2}=1$. Consequently the hardening function $\gamma(\delta)$ is equal to zero. From [20], the damage threshold, $\gamma_{\mathrm{o}}$, can easily be determined when the shear load is equal to $F_{6 E d}$ as

$$
\gamma_{0}=\sqrt{B_{22}} \frac{2 \overline{S_{66}}}{\Omega_{2 s}^{4}} F_{6 E d}^{2}
$$

where $\overline{S_{66}}=\frac{1}{\overline{G_{12}}}$.

In the same manner, when the virgin material is loaded with a pure shear stress bellow the in-plane shear elastic limit, $F_{6 E p}$, the internal unrecoverable-strain hardening material parameter $p$ is equal to zero and the unrecoverable-strains are also zero, consequently the hardening function $R(p)$ is equal to zero. From [20], the initial threshold value for unrecoverable-strain, $R_{0}$, can be determined

$$
R_{0}=\frac{\bar{F}_{6 E p}}{\bar{F}_{6}}
$$

where $\bar{F}_{6 E p}$ is the effective in-plane elastic limit

$$
\bar{F}_{6 E p}=\frac{1}{\Omega_{2 E p}} F_{6 E p}
$$

The internal material parameters for a epoxy fiberglass laminate containing $20 \%$ 
the shear elastic limit integrity $\Omega_{2 E p}$, defined in [20], can be obtained from the in-plane shear strain-stress constitutive response. Using a non-linear square minimum error method it is possible to obtain the parameters $c_{1}^{d}, c_{2}^{d}, c_{1}^{p}$ and $c_{2}^{p}$ as discussed in [20].

\subsection{Identification of Healing Parameters}

Unlike the damage parameters, which can be calculated from one experimental test, the healing parameters, $c_{1}^{h}, \ldots$, are calculated from a set of tests that measure the healing efficiency. Cyclic healing shear tests, discussed in Section 3.5, are preformed at various strain intervals. Several specimens are tested at $0.75 \%$ strain intervals, $1.5 \%$ strain intervals, $2 \%$ strain intervals, $2.5 \%$ strain intervals, $3.0 \%$ strain intervals, and up to $4 \%$ strain intervals. Next, the damage model is used to determine the amount of damage, $\mathrm{d}_{2}$, caused by the above mentioned strains. The healing efficiency for each reloading is then calculated using Eq. (4.49). The healing efficieny at each strain is then averaged and the healing efficiencies are then plotted as a function of damage, Figure 5.2. A fourth order polynomial equation is then fit to the averaged healing efficiencies. The terms that make up the fourth order polynomial define the healing efficieny parameters $c_{1}^{h}, c_{2}^{h}, c_{3}^{h}$, and $c_{4}^{h}$ and are tablulated in Table 5.3

\subsection{Comparison of SCDHM Model}

One limitation of the model is that plasticity cannot be turned off. After the specimen is loaded in the Iosipescu test fixture, the MTS machine crosshead is returned to zero and the specimen is removed from the fixture. When it is reloaded the specimen is placed back in the fixture and the strain gage is zeroed using the data aquistion software. Therefore, in order to model the Iosipescu shear test the SCDHM model needs 
to re-started with zero strain. This was done by changing the value of the unloading shear modulus, $G_{12}^{*}$, so that the unloading path returns to $\varepsilon=0$.

The SCDHM model was then fit to one specific specimen ([0/90] shear specimen) to determine if the model works. The shear modulus, $\mathrm{G}_{12}$, was calculated and the failure strength, $\mathrm{F}_{6}$, was assumed to be the largest value of shear stress the specimen endured, Table 5.4. Next, the model parameters were calculated for this specific specimen, Table 5.5 and Table 5.6. The healing efficiency of the specimen was also calculated, Table 5.4, and input into the SCDHM model. Figure 5.3 is a comparison the experimental data and SCDHM model prediction of the first loading of the specimen. Figure 5.4 is a comparison the experimental data and SCDHM model prediction of the second (healed) loading of the specimen. 
Table 5.1 Overall model parameters for damage and plasticity for laminates containing the self-healing system.

\begin{tabular}{lc}
\hline \multicolumn{2}{l}{ Property } \\
\hline$J_{11}$ & 0.0023030 \\
\hline$J_{22}$ & 0.0410099 \\
\hline$J_{33}$ & 0.0410099 \\
\hline$A_{1}$ & 1.32801 \\
\hline$A_{2}$ & 1 \\
\hline$A_{3}$ & 1 \\
\hline$B_{11}$ & 0 \\
\hline$B_{22}$ & 0.0611882 \\
\hline$B_{33}$ & 0.0611882 \\
\hline
\end{tabular}

Table 5.2 Overall adjustable parameters for damage and plasticity for laminates containing the self-healing system.

\begin{tabular}{ll}
\hline \multicolumn{2}{l}{ Property } \\
\hline $\mathrm{C}_{1}{ }^{\mathrm{d}}$ & 0.030807 \\
\hline $\mathrm{C}_{2}{ }^{\mathrm{d}}$ & -0.95384 \\
\hline$\gamma_{0}$ & 0.000582873 \\
\hline $\mathrm{C}_{1}{ }^{\mathrm{p}}$ & -0.434411 \\
\hline $\mathrm{C}_{2}{ }^{\mathrm{p}}$ & 0.0347361 \\
\hline $\mathrm{R}^{\circ}$ & 0.565589 \\
\hline
\end{tabular}

Table 5.3 Healing efficiency parameters

\begin{tabular}{lc}
\hline \multicolumn{2}{l}{ Property } \\
\hline $\mathrm{C}^{{ }^{\mathrm{h}}}$ & 3.8122 \\
\hline $\mathrm{C}_{2}{ }^{\mathrm{h}}$ & -5.3876 \\
\hline $\mathrm{C}_{3}{ }^{\mathrm{h}}$ & 1.539 \\
\hline $\mathrm{C}_{4}{ }^{\mathrm{h}}$ & 1 \\
\hline
\end{tabular}


Table 5.4 Shear properties for material in Figure 5.3 and Figure 5.4

\begin{tabular}{lcl}
\hline Property & & \\
\hline $\mathrm{G}_{12}{ }^{\circ}$ & 2147 & $\mathrm{MPa}$ \\
\hline $\mathrm{G}_{12}{ }^{\mathrm{h}}$ & 2129 & $\mathrm{MPa}$ \\
\hline $\mathrm{F}_{6}$ & 26.43 & $\mathrm{MPa}$ \\
\hline$\eta$ & $98.30 \%$ & \\
\hline
\end{tabular}

Table 5.5 Model parameters for damage and plasticity for the specimen in Figure 5.3 and Figure 5.4.

\begin{tabular}{lc}
\hline \multicolumn{2}{l}{ Property } \\
\hline$J_{11}$ & 0.00733065 \\
\hline$J_{22}$ & 0.130973 \\
\hline$J_{33}$ & 0.130973 \\
\hline$A_{1}$ & 1.29383 \\
\hline$A_{2}$ & 1 \\
\hline$A_{3}$ & 1 \\
\hline$B_{11}$ & 0 \\
\hline$B_{22}$ & 0.253744 \\
\hline$B_{33}$ & 0.253744 \\
\hline
\end{tabular}

Table 5.6 Overall adjustable parameters for damage and plasticity for the specimen in Figure 5.3 and Figure 5.4.

\begin{tabular}{ll}
\hline \multicolumn{2}{l}{ Property } \\
\hline $\mathrm{C}_{1}{ }^{\mathrm{d}}$ & 0.036242 \\
\hline $\mathrm{C}_{2}{ }^{\mathrm{d}}$ & -0.463645 \\
\hline$\gamma_{0}$ & 0.0015932 \\
\hline $\mathrm{C}^{\mathrm{p}}$ & -0.406213 \\
\hline $\mathrm{C}^{\mathrm{p}}{ }^{\mathrm{p}}$ & 0.0275111 \\
\hline $\mathrm{R}_{0}$ & 0.593787 \\
\hline
\end{tabular}




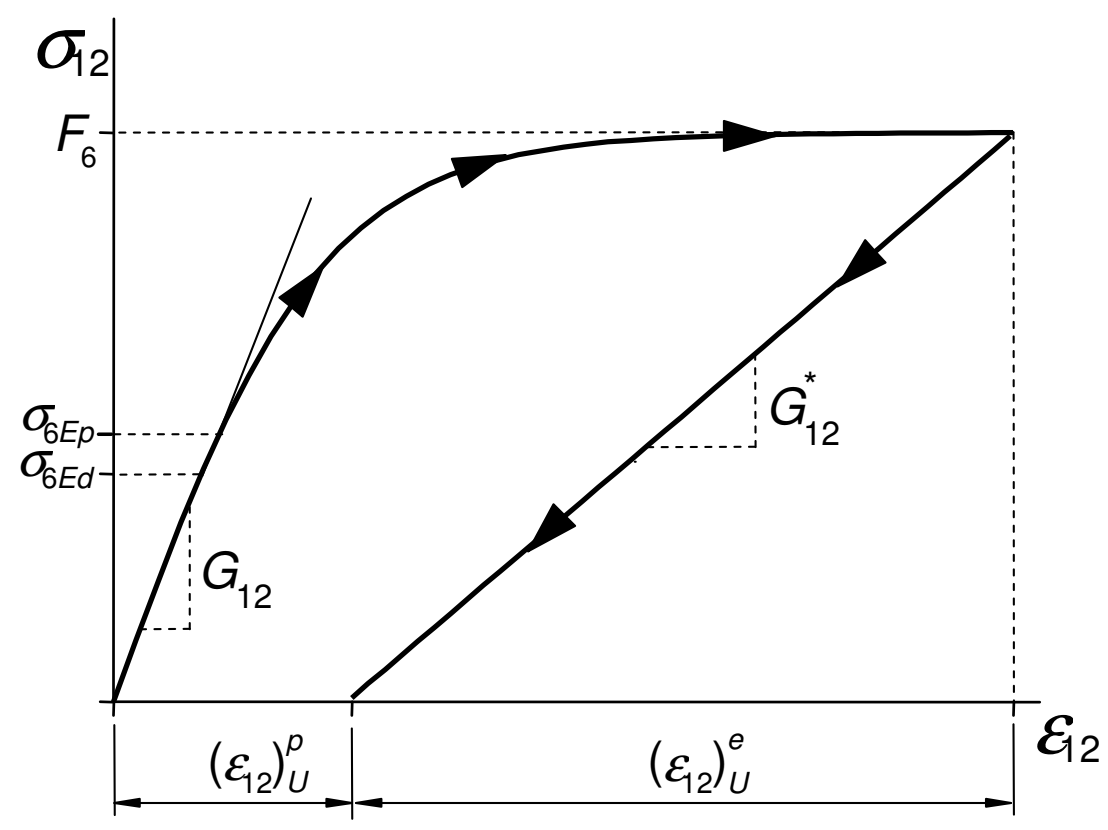

Figure 5.1 Typical shear stress versus shear strain loading and unloading curve.

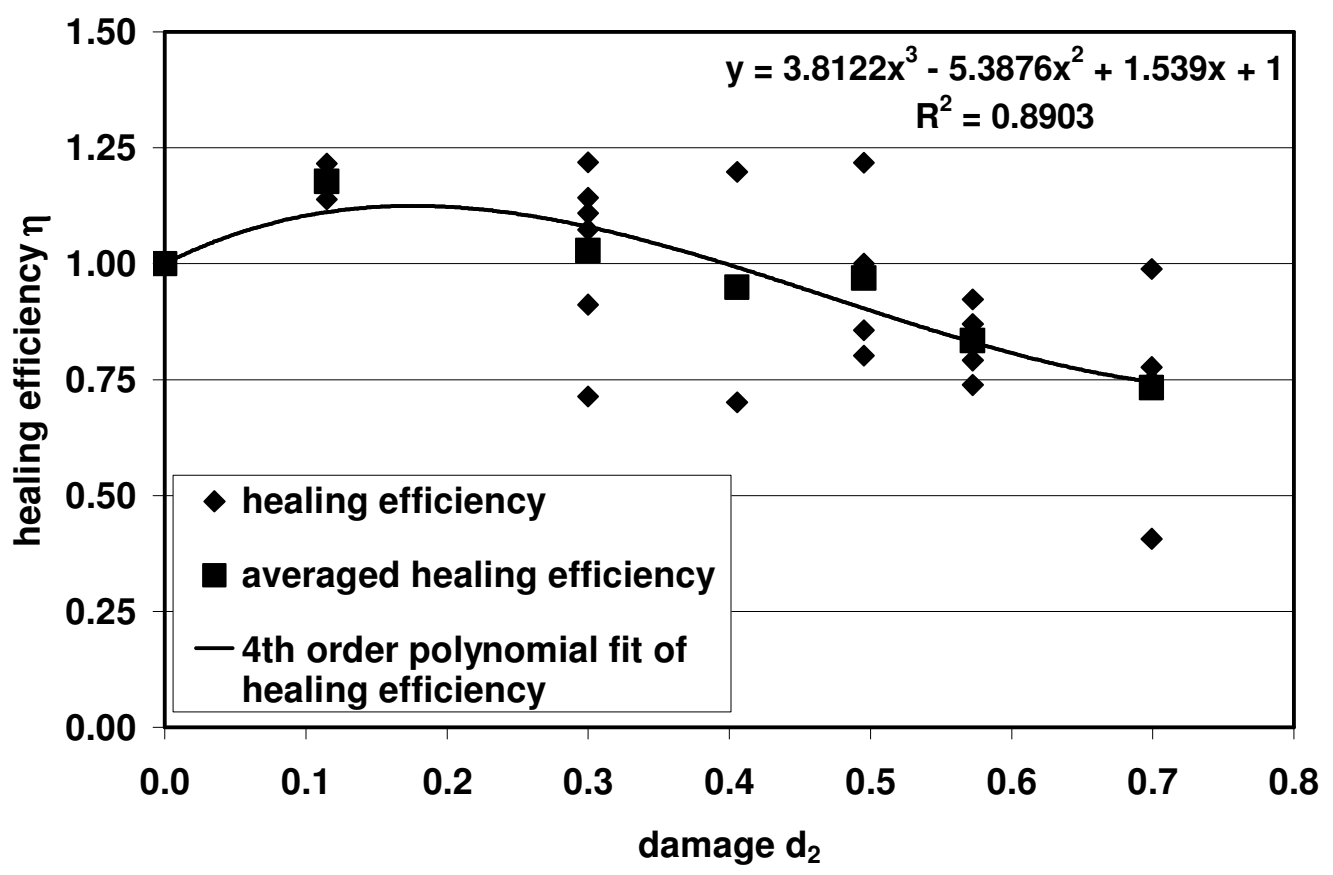

Figure 5.2 Healing efficiency versus transverse damage. 


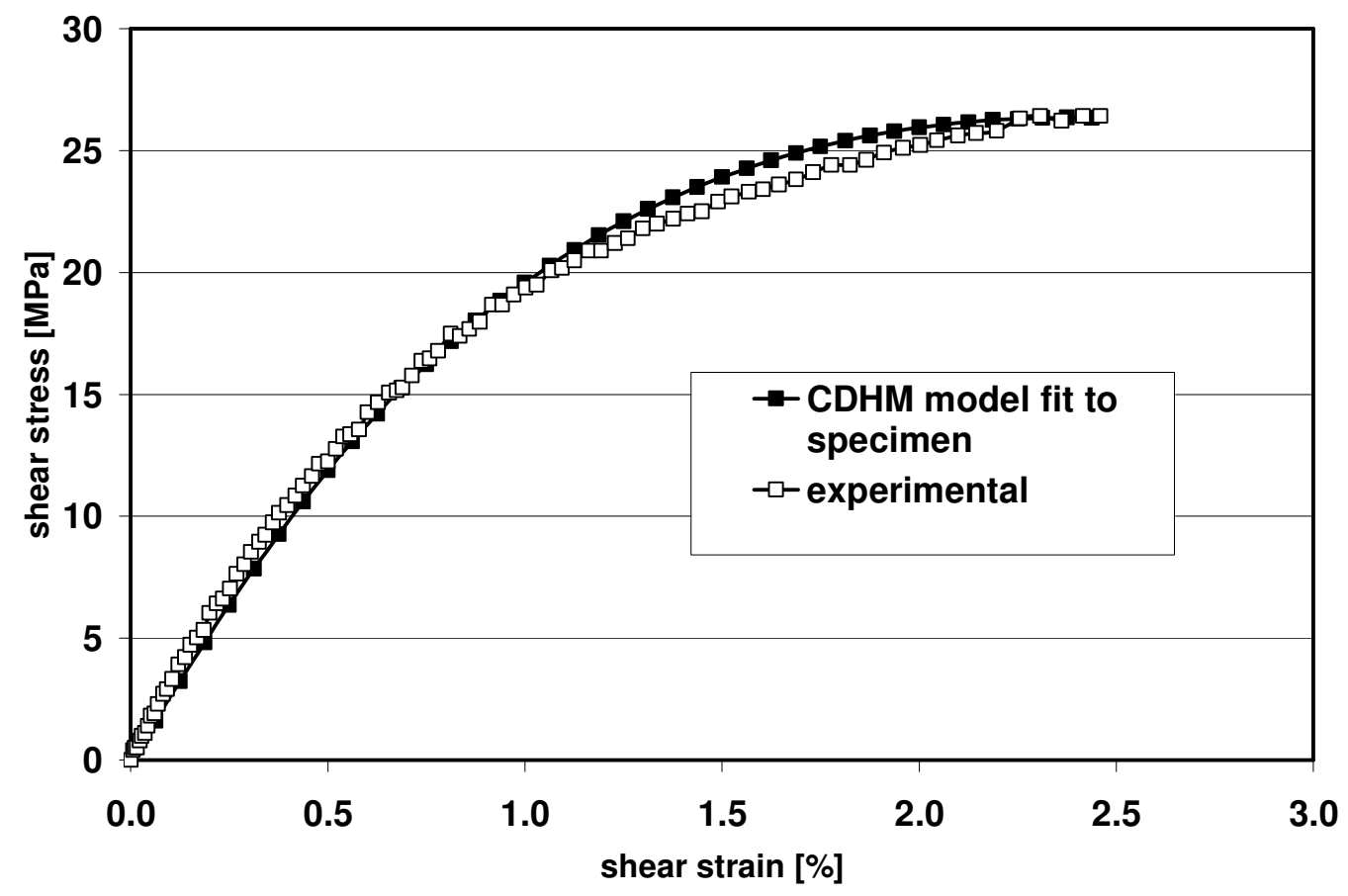

Figure 5.3 Comparison of CDHM model and experimental data for the first loading of a [0/90] laminate under shear loading.

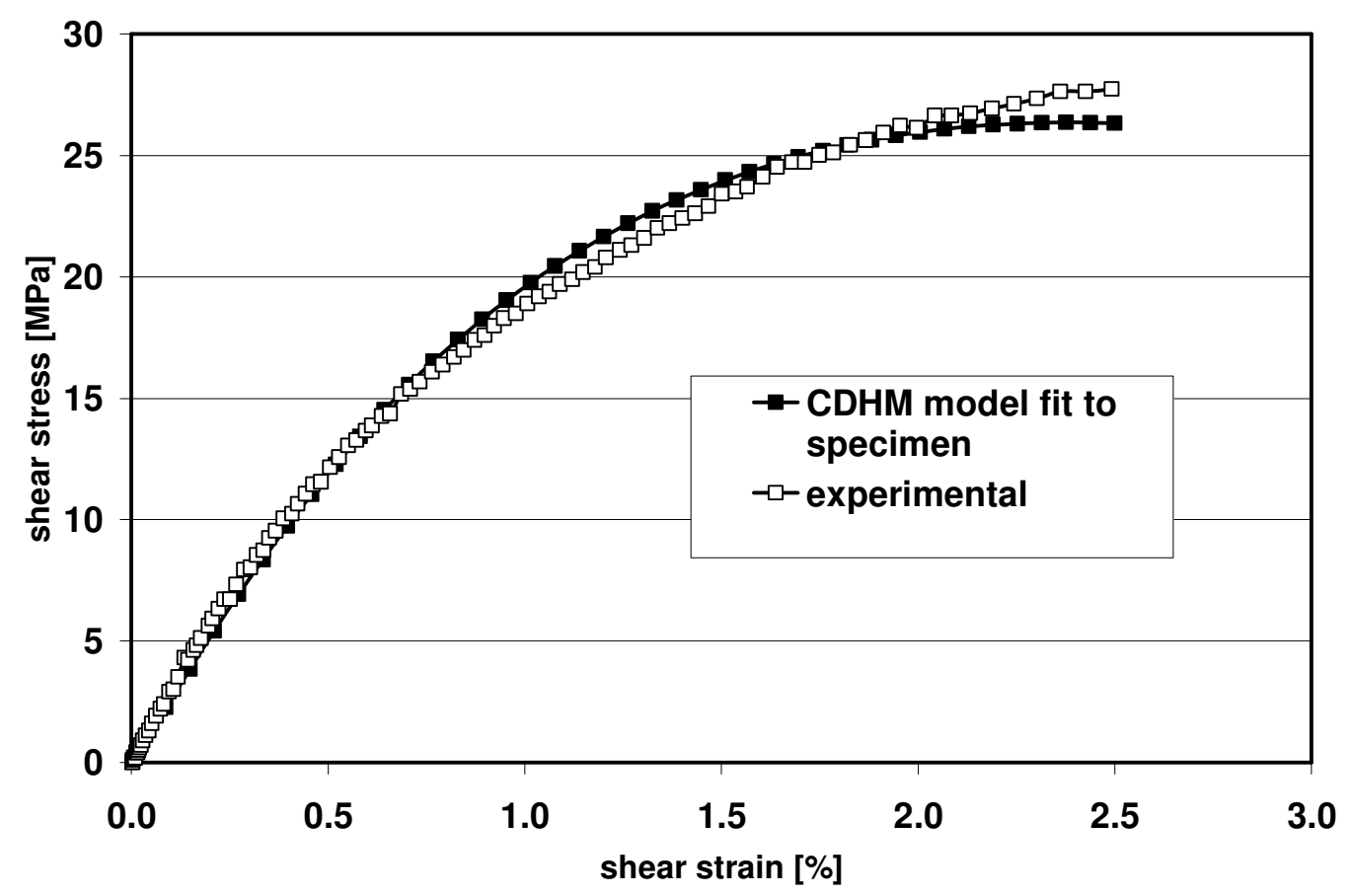

Figure 5.4 Comparison of CDHM model and experimental data for the second (healed) loading of a [0/90] laminate under shear loading. 


\section{MODEL VALIDATION}

Once the parameters for the Simplified Continuum Damage and Healing Mechanics model were determined (Table 5.1, Table 5.2, and Table 5.3), independent tests were conducted to validate the SCDHM model. In order to validate the SCDHM model shear tests of a quasi-isotropic, $\left[[0 / 90]_{3} /+45 /-45\right]_{s}$, laminate were preformed. These specimens were fabricated in the same manner outlined in Chapter 3. Cyclic healing test of three specimens were conducted at $1.5 \%$ strain increments and four specimens were tested at $2.25 \%$ strain increments. The experimental data was then fit with the following equation

$$
\tau=a+b \exp (-k \gamma)
$$

Next, shear stress strain curves were generated for each set of parameters fit with Eq. (6.1). For illustration purposes curves at $1.5 \%$ strain were averaged and the curves at $2.25 \%$ strain were averaged.

ANSYS was compiled with the SCDHM model user subroutine and was then used to model the tests. The dimensions of the model, which uses Solid 186 elements, are $2 \mathrm{~mm} \times 2 \mathrm{~mm} \times 8 \mathrm{~mm}$. Symmetry boundary conditions were applied so that only half of the laminate could be modeled (Figure 6.1). Local coordinate systems were used to define the orientation of each layer (Figure 6.2). Periodic boundary conditions that couple the nodes along opposite edges of the laminate were then applied to model the shear response of the laminate (Figure 6.3).

The constraint equations in the horizontal direction are

$$
\begin{gathered}
u_{1}\left(a_{1}, x_{2}, x_{3}\right)-u_{1}\left(-a_{1}, x_{2}, x_{3}\right)-2 a_{1} \varepsilon_{11}=0 \\
u_{2}\left(a_{1}, x_{2}, x_{3}\right)-u_{2}\left(-a_{1}, x_{2}, x_{3}\right)-2 a_{2} \varepsilon_{11}=0
\end{gathered}
$$


In the vertical direction the constraint equations are

$$
\begin{gathered}
u_{1}\left(x_{1}, a_{2}, x_{3}\right)-u_{1}\left(x_{1}, a_{2}, x_{3}\right)-2 a_{1} \varepsilon_{12}=0 \\
u_{2}\left(x_{1}, a_{2}, x_{3}\right)-u_{2}\left(x_{1}, a_{2}, x_{3}\right)-2 a_{2} \varepsilon_{22}=0
\end{gathered}
$$

On the $\mathrm{x}_{1}-\mathrm{x}_{2}$ plane the diagonally opposite vertices must also be constrained using the following constraint equations

$$
\begin{aligned}
& u_{i}\left(+a_{1},+a_{2}, x_{3}\right)-u_{i}\left(-a_{1},-a_{2}, x_{3}\right)-2 a_{1} \varepsilon_{i 2}=0 \\
& u_{i}\left(+a_{1},-a_{2}, x_{3}\right)-u_{i}\left(-a_{1},+a_{2}, x_{3}\right)-2 a_{1} \varepsilon_{i 2}=0
\end{aligned}
$$

In the case of the shear model used the following conditions are applied

$$
\begin{aligned}
& \varepsilon_{12} \neq 0 \\
& \varepsilon_{11}=0 \\
& \varepsilon_{22}=0
\end{aligned}
$$

Figure 6.4 is a comparison of the first loading and second (healed) loading of a $\left[[0 / 90]_{3} /+45 /-45\right]_{S}$ laminate loaded to $1.5 \%$ shear strain. Figure 6.5 is a comparison of the SCDHM model prediction and the first loading of the laminate in Figure 6.4. Figure 6.6 is a comparison of the SCDHM model prediction and the second (healed) loading of the laminate in Figure 6.4. Figure 6.7 is a comparison of the first loading and second (healed) loading of a $\left[[0 / 90]_{3} /+45 /-45\right]_{S}$ laminate loaded to $1.5 \%$ shear strain. Figure 6.8 is a comparison of the SCDHM model prediction and the first loading of the laminate in Figure 6.7 Figure 6.9 is a comparison of the SCDHM model prediction and the second (healed) loading of the laminate in Figure 6.7. 


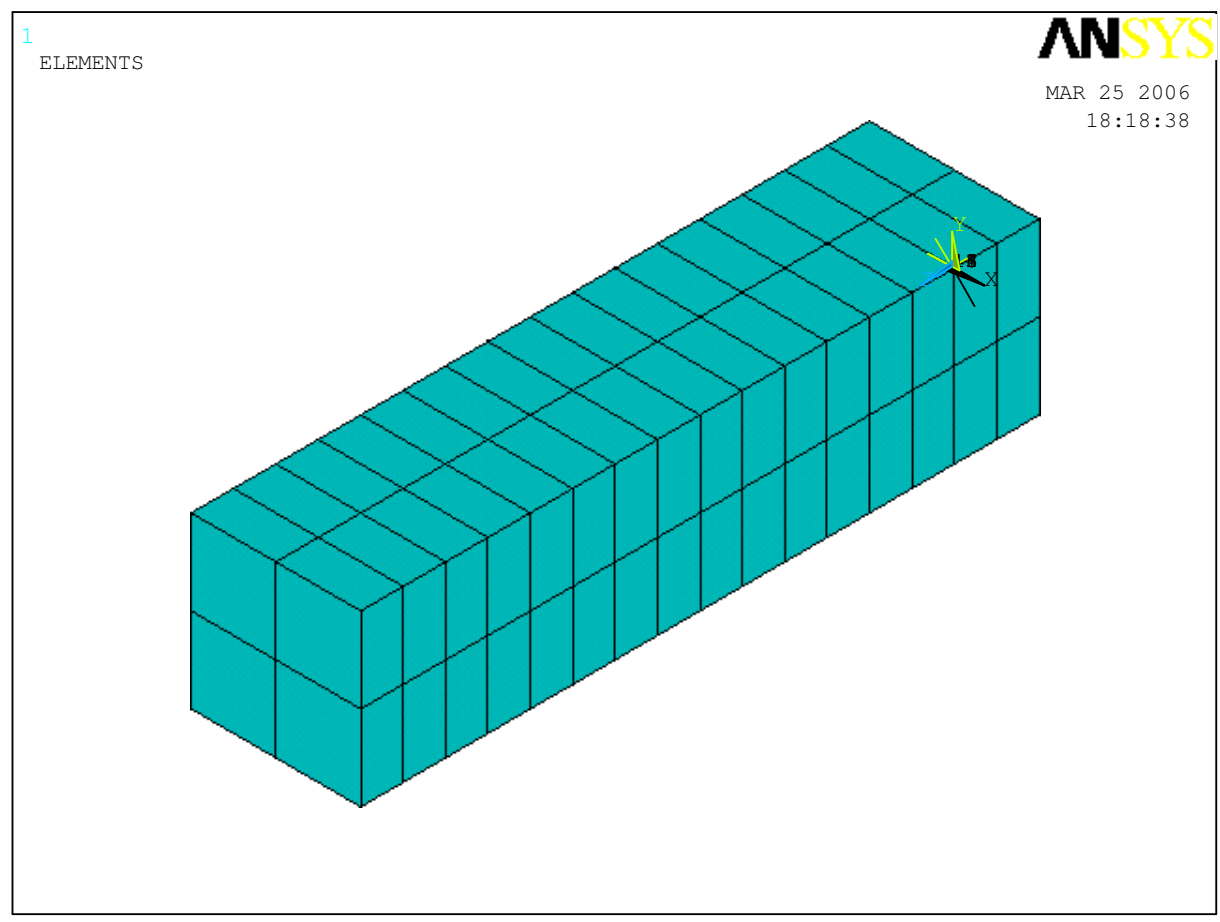

Figure 6.1 ANSYS model of a sixteen layer laminate using symmetry conditions.

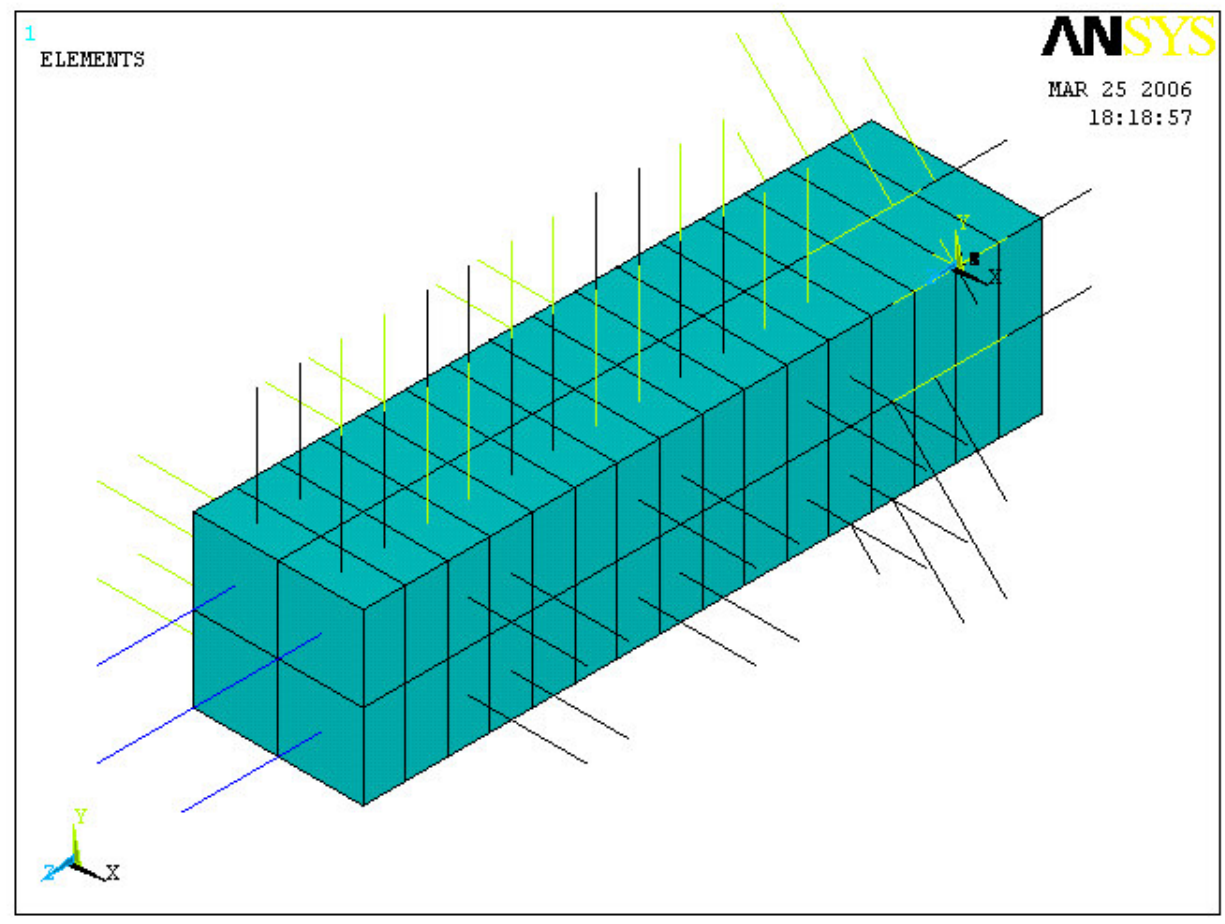

Figure 6.2 Orientation of layers. 


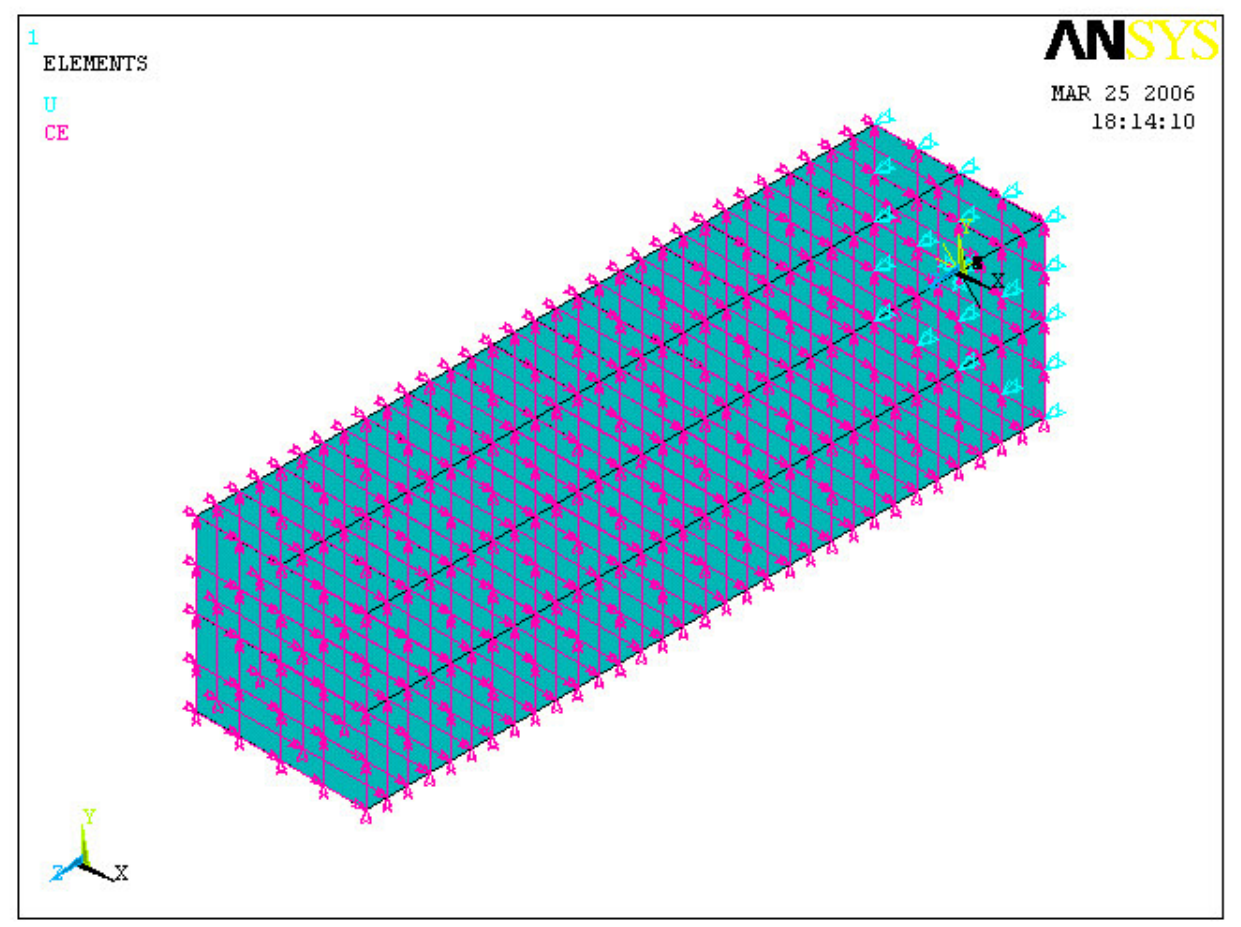

Figure 6.3 Coupling of nodes using periodic boundary conditions.

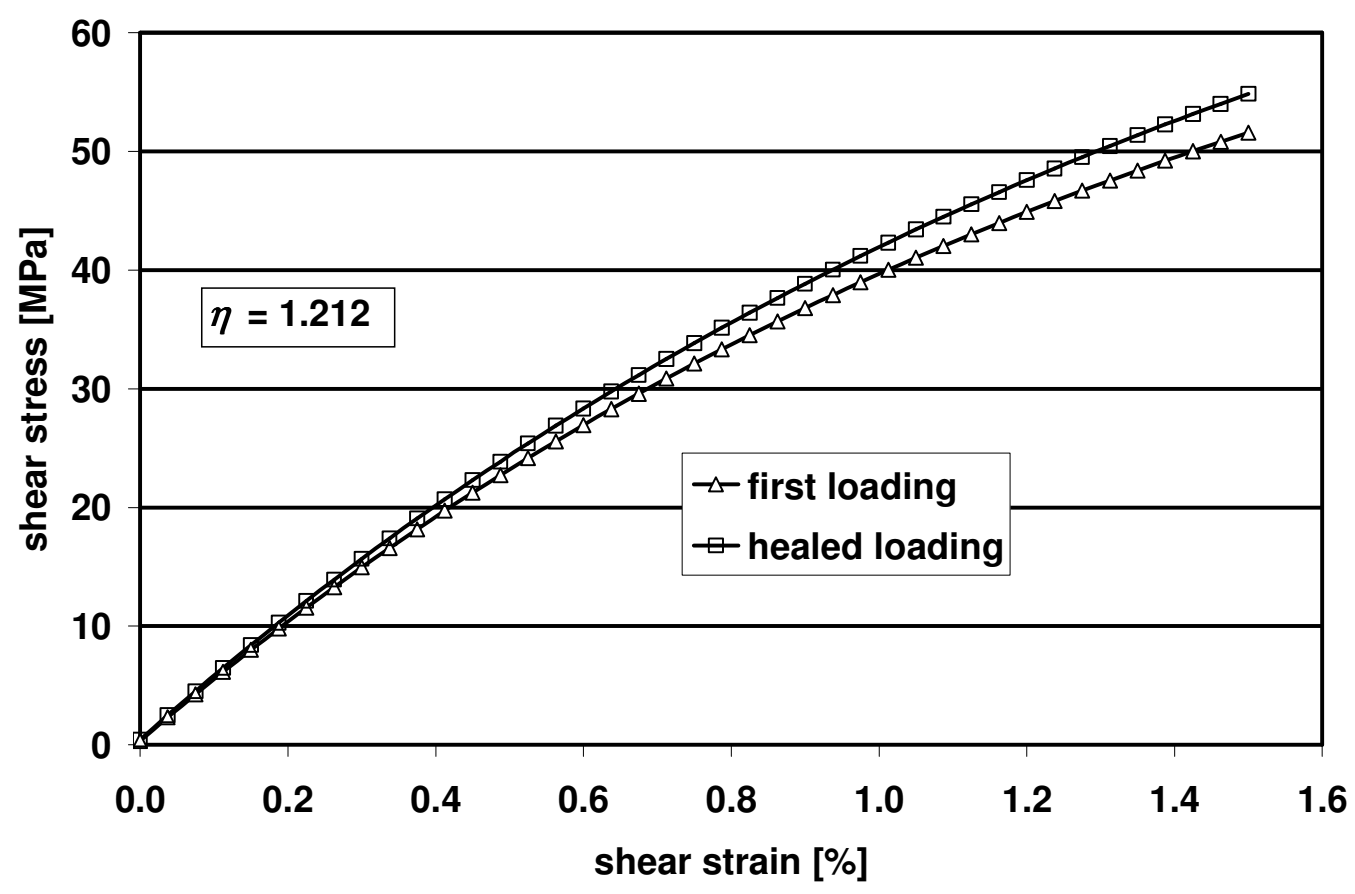

Figure 6.4 Comparison of the first loading and the second (healed) loading of a $\left[[0 / 90]_{3} /+45 /-45\right]_{S}$ laminate that was loaded to $1.5 \%$ shear strain. 


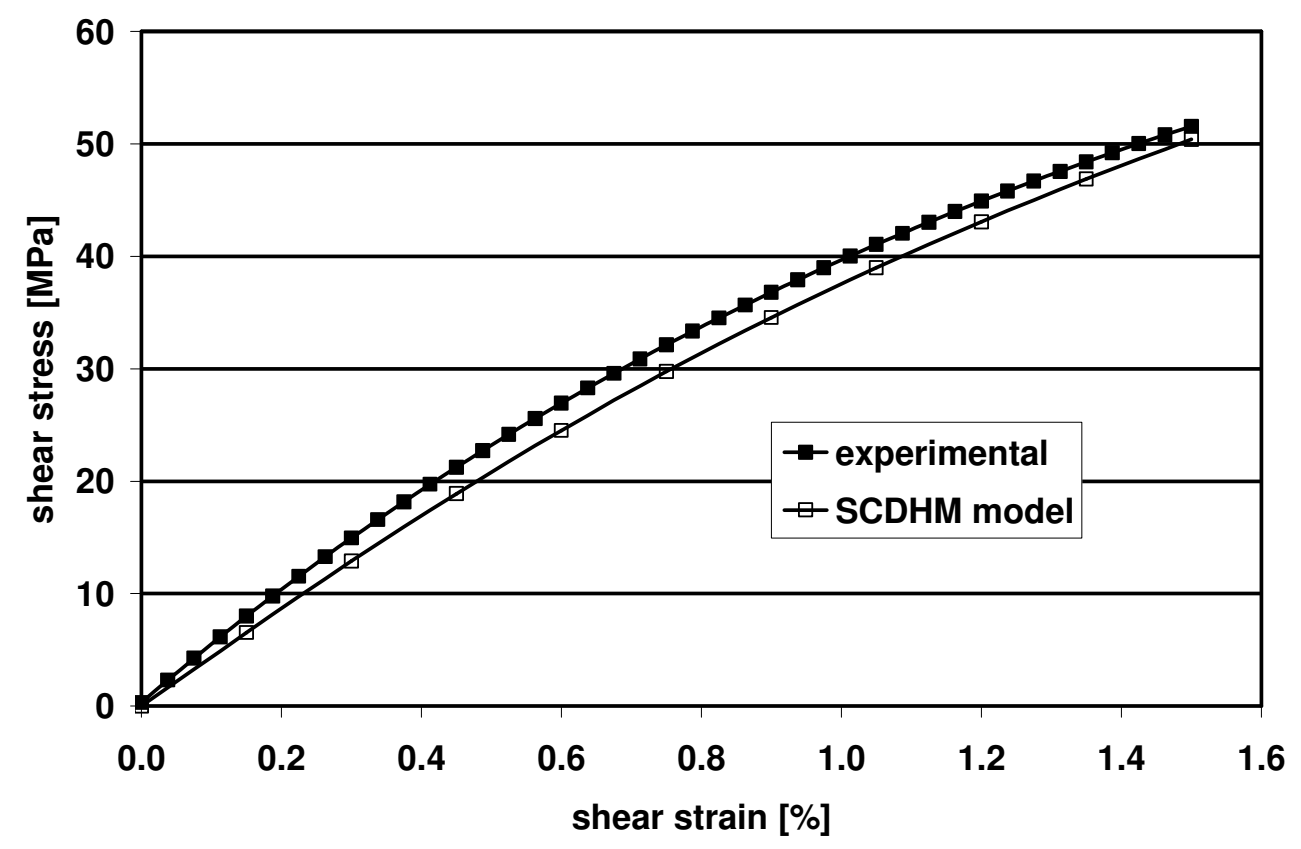

Figure 6.5 Comparison of the first loading of model and experimental data of a $\left[[0 / 90]_{3} /+45 /-45\right]_{S}$ laminate tested in cyclic healing loading at $1.5 \%$ strain increments.

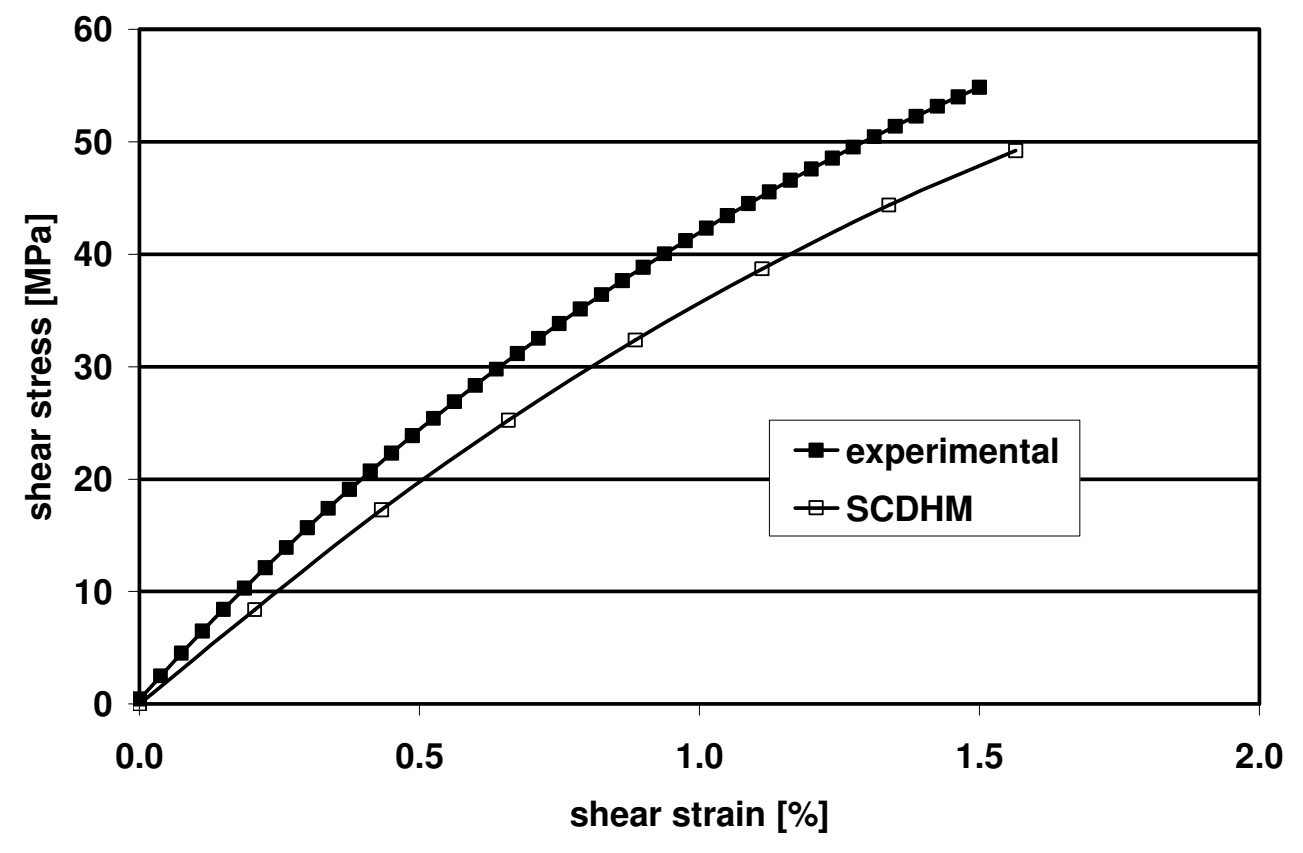

Figure 6.6 Comparison of the second (healed) loading of model and experimental data of a $\left[[0 / 90]_{3} /+45 /-45\right]_{S}$ laminate tested in cyclic healing loading at $1.5 \%$ strain increments. 


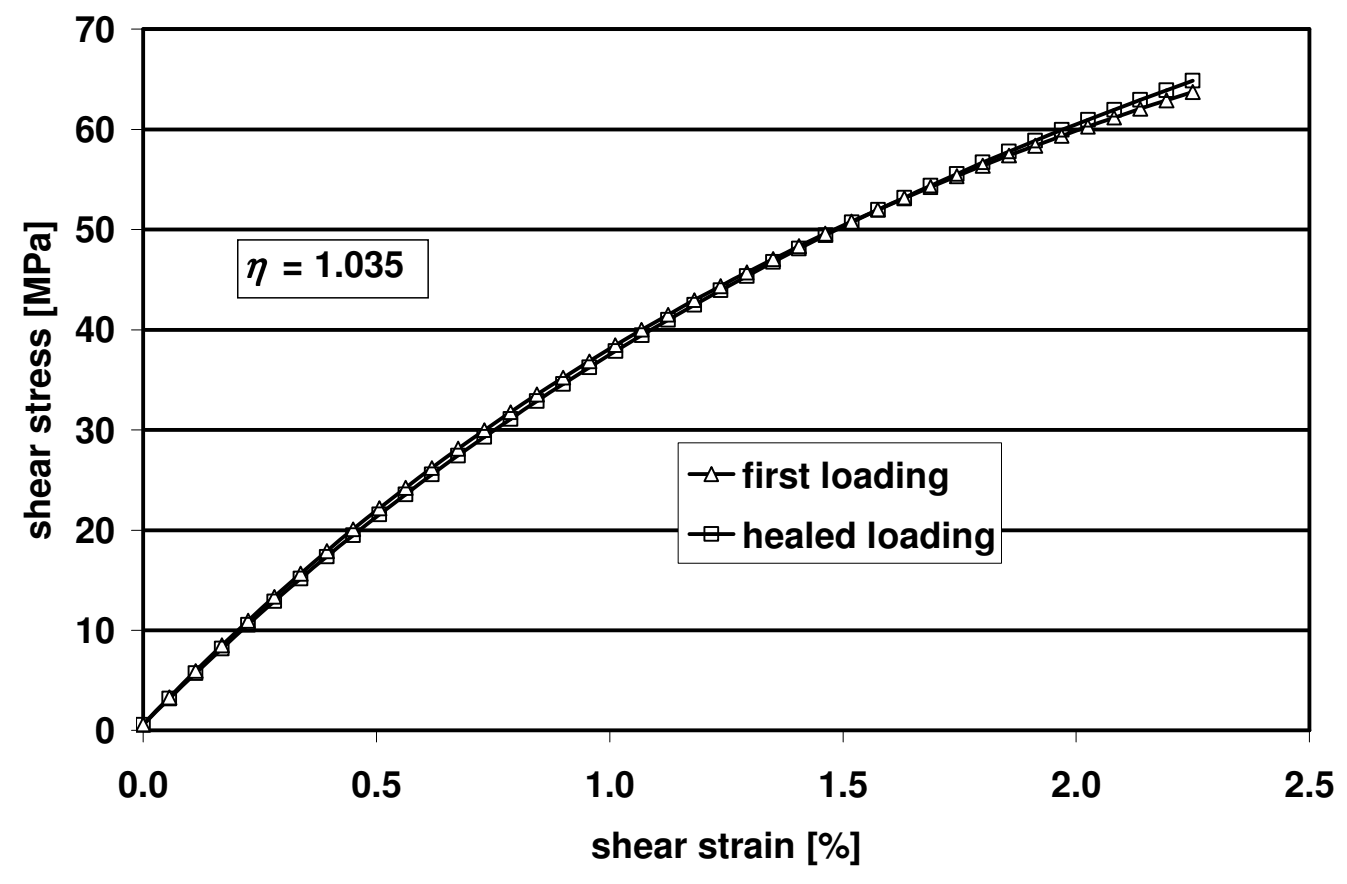

Figure 6.7 Comparison of the first loading and the second (healed) loading of a $\left[[0 / 90]_{3} /+45 /-45\right]_{S}$ laminate that was loaded to $2.25 \%$ shear strain.

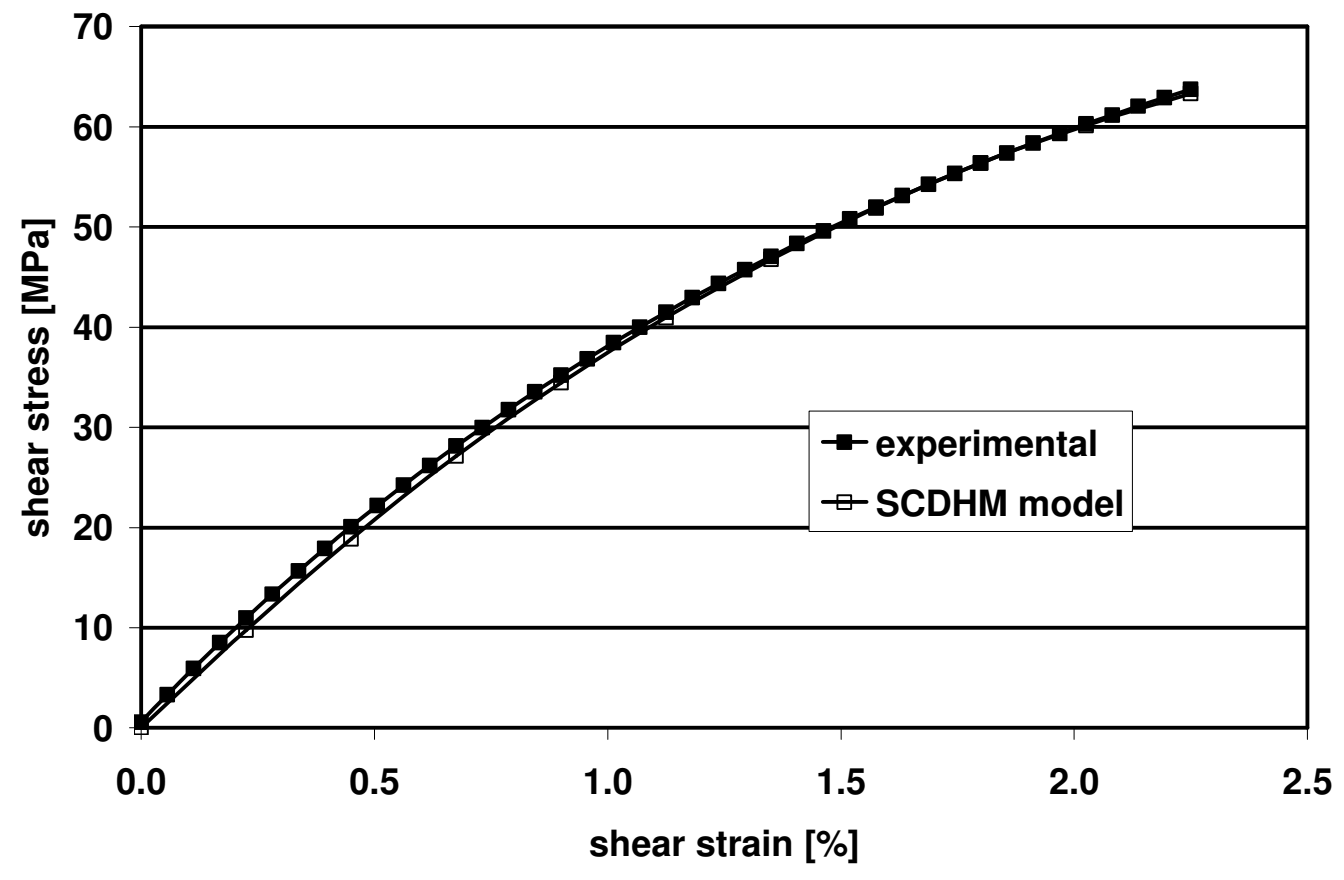

Figure 6.8 Comparison of the first loading of model and experimental data of a $\left[[0 / 90]_{3} /+45 /-45\right]_{S}$ laminate tested in cyclic healing loading at $2.25 \%$ strain increments. 


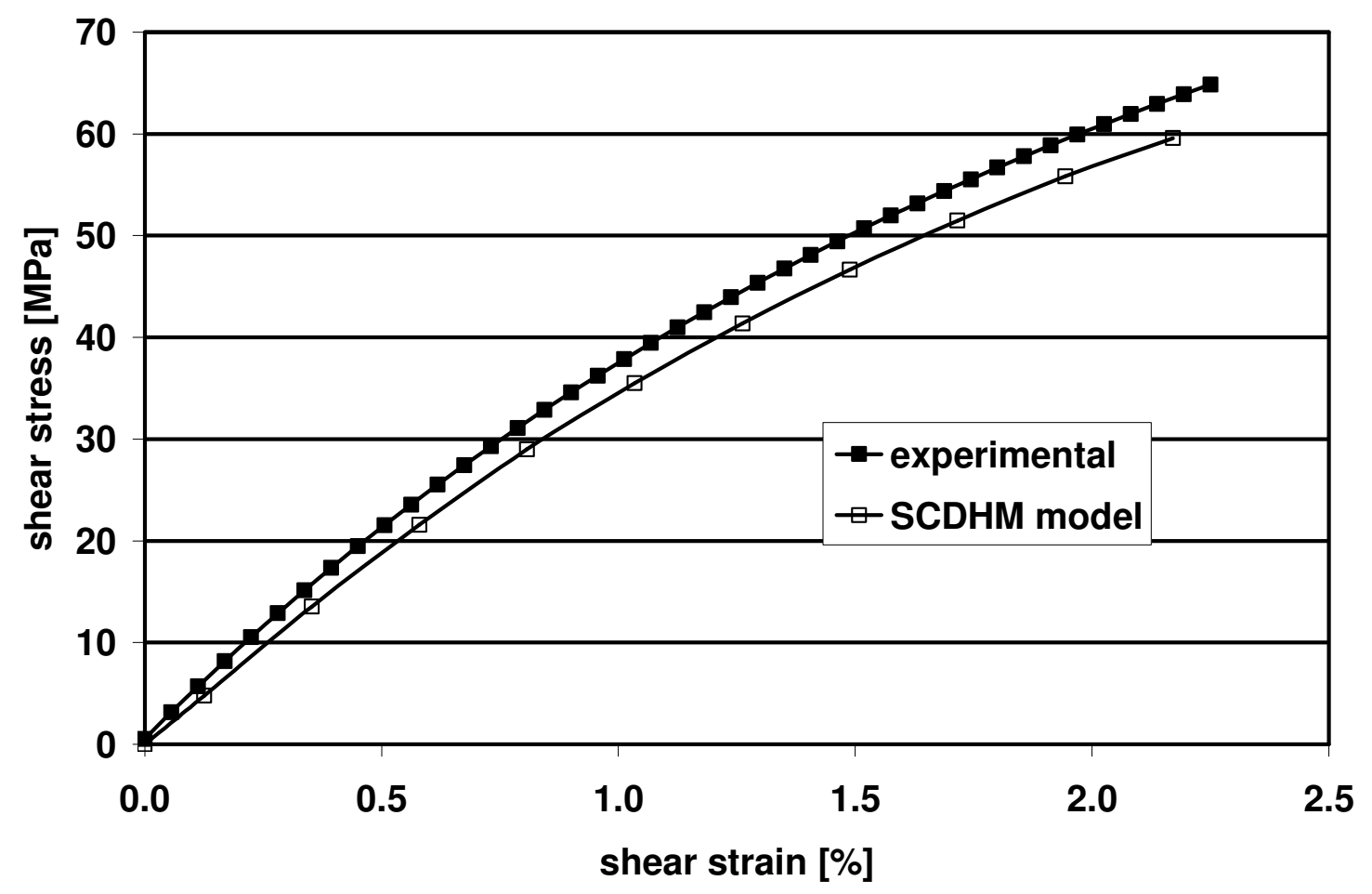

Figure 6.9 Comparison of the second (healed) loading of model and experimental data of a $\left[[0 / 90]_{3} /+45 /-45\right]_{S}$ laminate tested in cyclic healing loading at $2.25 \%$ strain increments. 


\section{DISCUSSION}

Before this project, the only existing body of literature that discussed the selfhealing of composite materials dealt with fracture toughness $[22,23,24,41,81]$. The current project dealt with one type of self-healing system and focused on using it to achieve measurable healing effects in shear loading. Several unknowns regarding selfhealing composite materials still exist. How does varying the concentration of the microcapsules and/or the catalyst affect the strength of the laminate and the healing efficiency of the laminate? What is the effect of the size of the microcapsules? Is it possible to develop a self-healing system that will cure quicker or stronger? How can self-healing composite materials be used in industry on large scale applications?

The overall objective of this research project was: a) to encapsulate DCPD and Grubbs' Ru catalyst, b) to successfully produce composites with self-healing in them, c) to demonstrate self-healing effects in fiber-reinforced laminated composites, d) to develop a computatuional model capable of predicting the observed behavior, e) to identify the model parameters from experimental data, and f) to develop/adapt existing experimental techniques capable of revealing the self-healing behavior. First, the selfhealing system developed by the University of Illinois Urbana Champaign was choosen. Urea and formaldehyde was used to encapsulate dicyclopentadiene, which acts as the healing agent in this system. Wax was then used to encapsulate Grubb's Ru catalyst to protect it from degrading. Tensile, compression, and shear specimens containing the selfhealing system were then fabricated. These specimens were tested in order to determine the materials properties, and to identify the damage model. Additional shear specimens 
were then fabricated and tested in order to determine the recovery or healing effects of the system, and to identify the healing portion of the model.

The CDM model [20] and CDHM model [19] were simplified to create the SCDHM model proposed here. The model was incorporated into ANSYS as a user material subroutine. For validation, additional specimens, $\left[[0 / 90]_{3} /+45 /-45\right]_{s}$, were fabricated and tested in cyclic healing shear loading. ANSYS in conjunction with the user-subroutine was used to model the validation tests. As can be seen in Figure 6.5, Figure 6.6, Figure 6.8, and Figure 6.9, the SCDHM does an adequate job predicting the healed reloading curves.

The accomplishments of this project are:

$\checkmark$ 1. Fabricated microcapsules in the same manner outlined by White et al. [81].

2. Wax-encapsulated Grubbs' RU catalyst in the same manner outlined by Rule et al. [71].

2. Conducted shear tests on specimens with microcapsules and catalyst to obtain measurable healing effects.

3. Developed a Simplified Continuum Damage and Healing Mechanics model.

4. Proposed and conducted tests to identify the healing parameters outlined in the improved SCDHM model in the 1-2 direction.

5. Implemented the SCDHM model in ANSYS.

6. Validated the SCDHM model by performing additional tests not used in the parameter identification. 


\section{REFERENCES CITED}

1. Aboudi J. (1991) Mechanics of Composite Materials: A Unified Approach, Elsevier.

2. Adam, J.L., A Simplified Model of Wound Healing (With Particular Reference to the Critical Size Defect), Mathematical and Computer Modelling, Volume: 30, Issue: 5-6, September, 1999, pp. 23-32

3. Alexandridou, S., Kiparissides, C., Mange, F., and Foissy, A., 2001, Surface characteriation of oil-containing polyterephthalamide microcapsules prepared by interfacial polymerization, Journal of Microencapsulation, 18, 767-781.

4. Ando, Kotoji; Chu, Min-Cheol; Tsuji, Kiichi; Hirasawa, Toshikazu; Kobayashi, Yasuyoshi; Sato, Shigemi, (2002a) Crack healing behaviour and high-temperature strength of mullite/SiC composite ceramics, Journal of the European Ceramic Society, 22:8,1313-1319

5. Ando, Kotoji; Chu, Min-Cheol; Tsuji, Kiichi; Hirasawa, Toshikazu; Kobayashi, Yasuyoshi; Sato, Shigemi, (2002b) Crack-healing behavior of $\mathrm{Si}_{3} \mathrm{~N}_{4} / \mathrm{SiC}$ ceramics under stress and fatigue strength at the temperature of healing $\left(1000{ }^{\circ} \mathrm{C}\right)$, Journal of the European Ceramic Society, 22:8,1339-1346

6. Arshady, R., and George, M. H., 1993, Suspension, dispersion, and interfacial polycondensation - a methodological survey, Polymer Engineering and Science, 33, 865-876.

7. ASTM D 3039/D 3039M - 00 Standard Test Method for Tensile Properties of Polymer Matrix Composite Materials

8. ASTM D 3410/D 3410M - 95 Standard Test Method for Compressive Properties of Polymer Matrix Composite Materials with Unsupported Gage Section by Shear Loading

9. ASTM D 3518/D 3518M - 94 (Reapproved 2001) Standard Test Method for InPlane Shear Response of Polymer Matrix Composite Materials by Tensile Test of a $\pm 45^{\circ}$ Laminate

10. ASTM D 5379/D 5379M - 98 Standard Test Method for Shear Properties of Composite Materials by the V-Notched Beam Method

11. Balazs, A. C., Tyagi, S., Buxton, G. A., and Lee, J. Y., Using Nanoparticles to Create Self-Healing Films, ASME/ASCE/ASCE/SES McMat Mechanics and Materials Conference, Baton Rouge, Louisiana, June 2005. 
12. Barbero, E. J., Prediction of Compression Strength of Unidirectional Polymer Matrix Composites, J. Composite Materials, 32 (5), 483-502, 1998.

13. Barbero, E. J., "Introduction to Composite Materials Design," Taylor and Francis, NY. 1999

14. Barbero, E. J. and Wen, E., Compressive Strength of Production Parts without Compression Testing, ASTM STP 1383 Compoiste Structures: Theory and Practice, 470-483, ASTM, PA, 2000.

15. Barbero, E. J. and DeVivo, L., A Constitutive Model for Elastic Damage in FiberReinforced PMC Laminae, J. of Damage Mechanics, 10(1) 73-93, 2001.

16. Barbero, E. J., Lonetti, P., Damage Model For Composites Defined in Terms of Available Data, Mechanics Of Composite Materials And Structures, 8(4), 299$315,2001$.

17. Barbero, E. J., Lonetti, P., An Inelastic Damage Model For Fiber Reinforced Laminates, Journal Of Composite Material, 36(8), 941-962, 2002.

18. Barbero, E. J., Lonetti, P., Raffaele Zinno, and Fabrizio Greco, Interlaminar Damage Model For Polymer Matrix Composites, J. Composite Materials, J. Composite Materials, 1485-1504, 37(16), 2003.

19. Barbero, E. J., Greco, E., Lonetti, P., Continuum Damage Healing Mechanics for Self-Healing Materials, 14(1):51-81, January 2005.

20. Mayugo, Joan A. and Barbero, Ever J., A Transversley Isotropic Damage Model with Unrecoverable-strain for Fiber Composites, submitted

21. Baxter, G., 1974, Microencapsulation processes in modern business forms. In Microencapsulation: Processes and Applications, edited by J. D. Vandegaer (New York, Plenum Press).

22. Brown, E. N., Kessler, M. R., Sottos, N. R., and White, S. R.: "In situ poly(ureaformaldehyde) Microencapsulation of dicyclopentadiene", Journal of Microencapsulation 2003 submitted.

23. Brown, E. N., Sottos, N. R., White, S. R. (2002) Fracture testing of self-healing polimeri composite, Experimental Mechanics, 42(4), 372-379.

24. Brown, E. N.; White, S. R. and Sottos, N. R.: "Microcapsule induced toughening in a self-healing polymer composite", Journal of Materials Science, 2004; 39, 1703-1710. 
25. Callister, William D. Jr., (2000) Materials Science and Engineering and Introduction, Fifth Edition, John Wiley and Sons, NY.

26. Chow C. L. and Wang J. (1987) An Anisotropy Theory of Elasticity For Continuum Damage Mechanics, Int. J. Fracture, v33, 3-16.

27. Cordebois J.L. and Sirodoff F.(1979) Damage-induced elastic anisotropyMechanics of Behavior of Anisotropic Solids/N295 Comportement Mechanique Des Solides Anisotropes, Martinus Nijhoff Publisher, pp.19-22, The Netherlands.

28. Davalos, J. F., Qiao, P., Wang, J., Salim, H. A., and Schlussel, J. "Shear Moduli of Structural Composites from Torsion Tests", Journal of Composite Materials Volume 36, Number 10, 2002.

29. Dietrich, K., Herma, H. Nastke, R., Bonatz, E., and Teige, W., 1989, Amino resin microcapsules, Acta Polymerica, 40, 243-251.

30. Ebeling, T., Hiltmer, A., Baer, E.: Delamination failure of a woven glass fiber composite. J Compos Mater 1997;31(13):1318-33.

31. Elzey, D. M., Design and Performance of SMA-Based Active Cellular Materials, ASME/ASCE/ASCE/SES McMat Mechanics and Materials Conference, Baton Rouge, Louisiana, June 2005.

32. Gipple, K. L. and Hoyns, D., Measurement of the out-of-plane response of thick section composite materials using the $\mathrm{V}$-notched beam specimen. Journal of Composite Materials, 28 (1994) 543-72.

33. Hansen N. and Schreyer H.L. (1994) A Thermodynamic Consistent Framework for Theories of Elastoplasticity Coupled With Damage, Int. J. Solids Structures, v31, 359-389.

34. Hayes, S. A., Jones, F. R. and Marshiya, K. Self-sensing and Healing Composite Materials, ASME/ASCE/ASCE/SES McMat Mechanics and Materials Conference, Baton Rouge, Louisiana, June 2005.

35. Herakovich, C. T. (1998) Mechanics of Fibrous Composites, John Wiley \& Sons, Inc. NY.

36. Jacobsen, S., Marchand, J., Boisvert, L., Effect of cracking and healing on chloride transport in OPC concrete, Cement and Concrete Research, Volume: 26, Issue: 6, June, 1996, pp. 869-881)

37. Jacobsen, S; Sellevold, E. J., Self healing of high strength concrete after deterioration by freeze/thaw, Vol: 26, Issue: 1 January, 1996 pp. 55-62 
38. Janson J. and Hult J., Damage Mechanics and Fracture Mechanics: A Combined Approach, J. Mec. Appl., 1, 69-84, 1997.

39. Jones, A. S., White, S. R. and Sottos, N. R., Controlled Kinetics for Optimized Autonomic Crack Healing, ASME/ASCE/ASCE/SES McMat Mechanics and Materials Conference, Baton Rouge, Louisiana, June 2005.

40. Ju, J.U, (1989) On Energy Based Coupled Elastoplastic Damage Theories: Constitutive Modeling And Computational Aspects, Int. J. Solids Structures, 25, 803-833.

41. Kessler, M. R. and White, S. R.; "Self-activated healing of delamination damage in woven composites", Composites Part A: applied science and manufacturing. 2001; 32:5 683-699.

42. Kessler, M. R. and White, S. R.; "Cure Kinetics of the Ring-Opening Metathesis Polymerization of Dicyclopentadiene", Journal of Polymer Science. Vol. 40, 2373-2383 (2002).

43. Kessler, M. R. (2002) Characterization and Performance of a Self-healing Composite Material. Doctor of Philosophy Dissertation, , University of Illinois Urbana Champaign, Il.

44. Kessler, M. R., Sottos, N. R., and White, S. R.: Self-healing structural composite materials, Composites Part A: applied science and manufacturing. 2003; 34:8, 743-753

45. Kessler, M. R. and Larin, G. E., Rheokinetics of Ring-Opening Metathesis Polymerization with Applications to Self-Healing Composites, ASME/ASCE/ASCE/SES McMat Mechanics and Materials Conference, Baton Rouge, Louisiana, June 2005.

46. Kim, Y., Davalos, J. F., and Barbero, E. J., "Progressive Failure Analysis of Laminated Composite Beams," J. Composite Materials, 30(5), 536-560, 1996.

47. Kachanov L. M. (1958) On the Creep Fracture Time, Izv. Akad Nauk USSR Otd. Tekh., v8, 26-31.

48. Kachanov L. M. (1992) Effective Elastic Properties of Cracked Solids: Critical Review of Some Basic Concepts, Appl. Mech. Rev., v45(8), 304-335.

49. Krajcinovic, D., Constitutive Equations for Damaging Materials, Journal of Applied Mechanics Vol. 50 pp. 355-360 1983. 
50. Ladeveze, P. and Dantec, E. Le, Damage modeling of the elementary ply for laminated composites. Composites Science and Technology 43 (1992) 257-267.

51. Lafarie-Frenot, M. C., and Touchard, F., Comparative In-plane Shear Behaviour of Long-Carbon-Fibre Composites with Thermoset or Thermoplastic Matrix, Composites Science and Technology 52 (1994) 417-425.

52. Lagace, Paul A., Nonlinear Stress-Strain Behavior of Graphite/Epoxy Laminates, AIAA Journal, Vol. 23, No. 10, October 1985 1583-1589.

53. Leknitskii, S. F. (1963). Theory of Elasticity of an Anisotropic Body. San Francisco, CA: Holden-Day Inc.

54. Lubliner, J. (1972) On the thermodynamic foundations of nonlinear solids mechanics. Int. J. Non-Linear Mech. v7, 237-254.

55. Maillard, S., de La Roche, C., Hammoum, F., and Such, C., Towards a better understanding of healing phenomenon in a thin film of bitumen, ASME/ASCE/ASCE/SES McMat Mechanics and Materials Conference, Baton Rouge, Louisiana, June 2005.

56. Maiti, S. and Geubelle, P. H., Numerical modeling of fatigue crack retardation in self-healing polymers, ASME/ASCE/ASCE/SES McMat Mechanics and Materials Conference, Baton Rouge, Louisiana, June 2005.

57. Mase G. E. and Mase G. T. (1992) Continuum Mechanics for Engineers, CRC Press, Boca Raton FL.

58. Miao, S., Wang, M. L., Schreyer, H.L. (1995) Constitutive models for healing of materials with application to compaction of crushed rock salt, Journal of Engineering Mechanics, 121, 12, 1122-1129.

59. Miao, S., and Wang, M. L., (1994), Mechanical properties of consolidated crushed rock salt", Proc., $4^{\text {th }}$ Annu, WERC Technl. Development Conf., WERC, Dept. of Energy, Las Cruces, N.M.

60. Morton, J., Ho, H., and Tsai, M. Y., An evaluation of the Iosipescu specimen for composite materials shear property measurement. Journal of Composite Materials, 26 (1992) 708-50.

61. Murakami S. and Ohmo N., (1981) A continuum theory of creep damage, in : A.R.S. Ponter, Creep in Structures, Springer, Berlin, pp. 422-444.

62. Murakami, S., Mechanical Modeling of Material Damage. Journal of Applied Mechanics Vol. 55 pp. 280-286 1988. 
63. Nemat-Nasser, S. and Amini, M. R., Self-healing Composites, ASME/ASCE/ASCE/SES McMat Mechanics and Materials Conference, Baton Rouge, Louisiana, June 2005.

64. Ogden R.W. (1984) Non-Linear Elastic Deformation, Dover Publications, Inc., Mineola, NY.

65. Ovez, B., Citak, B., Oztemel, D., Balbas, A., Peker, S., and Cakir, S., 1997, Variation of droplet sizes during the formation of microspheres from emulsions, Journal of Microencapsulation, 14, 489-499.

66. Pierron, F. and Vautrin, A., Accurate comparative determination of the in-plane shear modulus of T300/914 by the Iosipescu and $45^{\circ}$ off-axis tests. Composite Science Technology, 52 (1994) 61-72.

67. Piggott, M. R., Liu, K., and Wang, J., (2000) New Experiments Suggest that all the Shear and some Tensile Failure Processes are Inappropriate Subjects for ASTM Standards, ATSM STP Compoiste Structures: Theory and Practice.

68. Ramm, W., Biscoping, M., Autogenous healing and reinforcement corrosion of water-penetrated separation cracks in reinforced concrete, Nuclear Engineering and Design 179 (1998) 191-200.

69. Rosen B. W., The Tensile Failure of Fibrous Composites, AIAA Journal, 2 (11), 1985-1911, 1964.

70. Rule, J. D. and Moore, J. S. "ROMP Reactivity of endo- and exoDicyclopentadiene", Journal American Chemical Society. 2002

71. Rule, J., Brown, E.N., Sottos, N.R., White, S.R. and Moore, J.S. "Wax-protected catalyst microspheres for efficient self-healing materials", Advanced Materials, 72, 205-208 (2005).

72. SACMA SRM-1R-94 Compression test

73. SACMA SRM 4R-94 Tensile test

74. Schwab, P., Grubbs, R. H. and Ziller, J. W. "Synthesis and Applications of RuCl2(=CHR')(PR3)2: The Influence of the Alkylidene Moiety on Metathesis Activity", Journal American Chemical Society. 1996: 118, 100-110.

75. Sikil, K. K. Finite Element Damage Modeling of Plain Weave Fabrics, Masters of Science Thesis, West Virginia University, 2003 
76. Simpson, A.H.R.W., Gardner, T. N., Evans, M., Kenwright, J., Stiffness, strength and healing assessment in different bone fractures - a simple mathematical model (Injury, Volume: 31, Issue: 10, December, 2000, pp. 777 - 781.

77. Tan, H. S., Ng, T. H., and Mahabadi, H. K., 1991 Interfacial polymerization encapsulation of a viscous pigment mix - Emulsification conditions and particlesize distribution, Journal of Microencapsulation, 8, 525-536.

78. Thies, C., 1987, Microencapsulation. In Encyclopedia of Polymer Science and Engineering, Vol. 9, $2^{\text {nd }}$ Ed., edited by J. F. Mark, N. Bikales, C. G. Overberger, G. Menges, J. I. Kroschwitz (New York, Chichester : Wiley).

79. Trask, R. S. and Bond, I. P., A Self-healing Repair System for Composite Structures in a Space Environment, ASME/ASCE/ASCE/SES McMat Mechanics and Materials Conference, Baton Rouge, Louisiana, June 2005.

80. Wen, Ed: "Compressive Strength Prediction for Composite Unmanned Aerial Vehicles", Thesis West Virginia University. 1999

81. White, S. R.; Sottos, N. R.; Geubelle, P. H.; Moore, J.S.; Kessler, M. R.; Sriram, S. R.; Brown, E. N. and Viswanathan, S.: "Automatic healing of polymer composites", Nature. 2001: 409, 794-797.

82. White, S. R., Sottos, N. R., Moore, J. S., and Geubelle, P. H., "Automatic healing of polymer composites."

83. Whitney, J. M. (1993). Analysis of anisotropic laminated plates subjected to torsional loading. Composites Engineering, 3(1): 83-97.

84. Wool, R. P. and O'Conner, K. M., "A Theory of Crack Healing in Polymers," J. Appl. Phys., 52, 5953-5963 (1982)

85. Yan, N., Ni, P., and Zhang, M., 1993, Preparation and properties of polyurea microcapsules with nonionic surfactant as emulsifier, Journal of Microencapsulation, 10, 375-383.

86. Yin, W. L., A New Theory of Kink Band Formation, AIAA-92-2552-CP, 1992.

87. Voyiadjis, G. Z. and Park, T. Local and Interfacial Damage Analysis of Metal Matrix Composites. International Journal of Engineering Science, Vol. 33, No. 11 pp. 1595-1621, 1995.

88. Voyiadjis, G. Z. and Park, T. Anisotropic Damage of Fiber-Reinforced MMC Using Overall Damage Analysis. Journal of Engineering Mechanics, Nov. 1995, pp. 1209-1217. 
89. Voyiadjis, G. Z. and Deliktas, B. A coupled anisotropic damage model for the inelastic response of composite materials. Computer Methods Appl. Mech. Engrg. 183 (2000) 159-199.

90. Voyiadjis, G. Z. and Venson A. R., Experimental Damage Investigation of a SiCTi Aluminide Metal Matrix Composite, Int. J. Solids Structures, 4, 338-361, 1995.

91. Yurgartis, S. W. and Sternstein, S. S., Experiments to Reveal the Role of Matrix Properties and Composite Microstructure in Longitudinal Compression Response of Composite Structures, ASTM, Nov. 16-17, 1992.

92. Zhou, G., Green, E. R., and Morrison, C. "In-plane and Interlaminar Shear Properties of Carbon/Epoxy Laminates", Composites Science and Technology, 55 (1995) 187-193.

93. Zhu, Y. Y., A Fully Coupled Elasto-Visco-Plastic Damage Theory for Anisotropic Materials, International Journal of Solids Structures Vol. 32. No. 11 pp. 1607-1641, 1995. 


\section{APPENDIX A MICROENCAPSULATION OF DCPD INSTRUCTIONS}

1. Distillation of dicyclopentadiene (DCPD)

a. Place two scoops, approximately 50 grams of neutral activated aluminum oxide (Aldrich Chemical Company) into a round bottom flask

b. Place approximately $120 \mathrm{ml}$ of DCPD (Fisher Scientific) into the same flask

c. Attach the flask to the bottom of a distillation system and lower it into a silicon bath

d. Place a partial vacuum on the distillation process

e. Heat the silicon bath to $110^{\circ} \mathrm{C}$ while the flask is slowly rotating

f. Collect the first 10-20 $\mathrm{ml}$ of distilled DCPD and discard

g. Collect the next 70-80 $\mathrm{ml}$ of distilled DCPD and place in a cold room

h. Discard the remaining undistilled DCPD

2. Prepare the following solutions

a. $2.5 \mathrm{wt} \%$ aqueous solution of Ethylene Maleic Anhydride (EMA) Copolymer (Zeeland Chemicals) - the EMA takes several hours to dissolve in water under constant heat and continuous agitation

b. $37 \mathrm{wt} \%$ aqueous solution of formaldehyde (Fisher Scientific)

3. Place $200 \mathrm{ml}$ of deionized water into a $1000 \mathrm{ml}$ beaker suspended in a temperature controlled water bath

4. Bring the water to room temperature approximately $20^{\circ} \mathrm{C}$

5. Place $50 \mathrm{ml}$ of $2.5 \mathrm{wt} \%$ aqueous EMA solution into the beaker

6. Begin agitation of the solution to the desired RPM setting with a digital mixer (Eurostar, IKA Labortechnik) driving a three-bladed $63.5 \mathrm{~mm}$ diameter low-shear propeller (Cole Parmer)

7. Add $5.00 \mathrm{~g}$ of urea (Fisher Scientific) to the solution

8. Add $0.50 \mathrm{~g}$ of ammonium chloride (Fisher Scientific) to the solution

9. Add $0.50 \mathrm{~g}$ of resorcinol (J.T. Baker) to the solution

10. Raise the $\mathrm{pH}$ from approximately 2.7 to 3.75 by the addition of dilute sodium hydroxide $(\mathrm{NaOH})$ (Fisher Scientific)

11. Lower the $\mathrm{pH}$ to 3.5 by the addition of dilute hydrochloric acid $(\mathrm{HCl})$ (Fisher Scientific)

12. Add a few drops of 1-octanol (J.T. Baker) to eliminate surface bubbles

13. $60 \mathrm{ml}$ of DCPD is then added in a slow steady stream and allow it to stabilize for 10 minutes

14. Add $12.67 \mathrm{~g}$ of $37 \mathrm{wt} \%$ aqueous solution of formaldehyde

15. Cover the beaker with aluminum foil and raise the heat by $1{ }^{\circ} \mathrm{C} / \mathrm{min}$ to $55^{\circ} \mathrm{C}$

16. After two hours of continuous agitation at $55^{\circ} \mathrm{C}$ add an additional $50 \mathrm{ml}$ of deionized water to the solution

17. After four total hours of continuous agitation at $55^{\circ} \mathrm{C}$ turn off the mixer and hot plate and allow to cool to room temperature

18. Separate the suspension of microcapsules under vacuum with a coarse-fritted filter and rinse them with at least $1000 \mathrm{ml}$ of deionized water

19. Allow the capsules to dry 


\section{APPENDIX B COMPARISON OF ENCAPSULATION METHODS}

The wordings of the different encapsulation process are noted and the differences in numerical values are in bold.

\begin{tabular}{|c|c|c|c|}
\hline Kessler Dissertation & Brown et al. & White et al. & Ford/Stiller attempt \\
\hline $600 \mathrm{ml}$ beaker & $1000 \mathrm{ml}$ beaker & $600 \mathrm{ml}$ beaker & $1000 \mathrm{ml}$ beaker \\
\hline $200 \mathbf{~ m l}$ deionized water & $200 \mathrm{ml}$ deionized water & $150 \mathbf{~ m l}$ deionized water & $200 \mathrm{ml}$ deionized water \\
\hline $\begin{array}{l}\mathbf{5 0 ~} \mathbf{~ m l} 2.5 \% \text { wt. solution of } \\
\text { ethylene maleic anhydride } \\
\text { copolymer }\end{array}$ & $\begin{array}{l}\mathbf{5 0 ~} \mathbf{~ m l ~} 2.5 \% \text { wt. solution of } \\
\text { ethylene maleic anhydride } \\
\text { copolymer }\end{array}$ & $\begin{array}{l}\mathbf{1 0 0} \mathbf{~ m l ~ 5 \% ~ w t . ~ s o l u t i o n ~ o f ~} \\
\text { ethylene maleic anhydride } \\
\text { copolymer }\end{array}$ & $\begin{array}{l}\mathbf{5 0 ~} \mathbf{~ m l ~} 2.5 \% \text { wt. solution of } \\
\text { ethylene maleic anhydride } \\
\text { copolymer }\end{array}$ \\
\hline Constant agitation & Constant agitation & & $\begin{array}{l}\text { Constant agitation, mixer at } \\
\text { desired speed }\end{array}$ \\
\hline $\begin{array}{l}\text { Propeller just above bottom of } \\
\text { beaker }\end{array}$ & $\begin{array}{l}\text { Propeller just above bottom of } \\
\text { beaker }\end{array}$ & & $\begin{array}{l}\text { Propeller just above bottom of } \\
\text { beaker }\end{array}$ \\
\hline 5.0 g urea & $5.00 \mathrm{~g}$ urea & 7.00 g urea & 5.00 g urea \\
\hline $\begin{array}{l}\text { Brought back to } 3.5 \text { with few } \\
\text { drops of } \mathrm{HCl}\end{array}$ & & & $\mathrm{pH}$ lowered to 3.50 by $\mathrm{HCl}$ \\
\hline Add few drops of 1-octanol & Add few drops of 1-octanol & & Add few drops of 1-octanol \\
\hline \multicolumn{4}{|l|}{ Mixer set to desired speed } \\
\hline $\begin{array}{l}60 \mathrm{ml} \text { of DCPD is slowly } \\
\text { poured into by a steady stream }\end{array}$ & $\begin{array}{l}60 \mathrm{ml} \text { of DCPD is slowly } \\
\text { poured into by a steady stream }\end{array}$ & $60 \mathrm{ml}$ of DCPD & $\begin{array}{l}60 \mathrm{ml} \text { of DCPD is slowly poured } \\
\text { into by a steady stream }\end{array}$ \\
\hline
\end{tabular}




\begin{tabular}{|c|c|c|c|}
\hline $\begin{array}{l}\text { DCPD is immiscible in water, } \\
\text { forms small droplets in the } \\
\text { solution }\end{array}$ & & & \\
\hline $\begin{array}{l}\text { Mixed for 5-10 minutes so the } \\
\text { DCPD droplets can stabilize }\end{array}$ & $\begin{array}{l}\text { Mixed for } 10 \text { minutes so the } \\
\text { DCPD droplets can stabilize }\end{array}$ & & $\begin{array}{l}\text { Mixed for } 10 \text { minutes so the } \\
\text { DCPD droplets can stabilize }\end{array}$ \\
\hline $\begin{array}{l}\mathbf{1 2 . 6 7} \mathbf{g} \text { of } 37 \% \text { wt. aqueous } \\
\text { solution of formaldehyde is } \\
\text { added (forms } 1: 1.9 \text { molar ratio } \\
\text { of formaldehyde to urea) }\end{array}$ & $\begin{array}{l}\mathbf{1 2 . 6 7} \mathbf{g} \text { of } 37 \% \text { wt. aqueous } \\
\text { solution of formaldehyde is } \\
\text { added (forms } 1: 1.9 \text { molar ratio } \\
\text { of formaldehyde to urea) }\end{array}$ & $\begin{array}{l}\mathbf{1 8 . 9 1} \mathbf{g} \text { of } 37 \% \text { wt. aqueous } \\
\text { solution of formaldehyde is } \\
\text { added (forms } 1: 1.9 \text { molar ratio } \\
\text { of formaldehyde to urea) }\end{array}$ & $\begin{array}{l}\mathbf{1 2 . 6 7} \mathbf{g} \text { of } 37 \% \text { wt. aqueous } \\
\text { solution of formaldehyde is } \\
\text { added (forms } 1: 1.9 \text { molar ratio of } \\
\text { formaldehyde to urea) }\end{array}$ \\
\hline Beaker is covered with $\mathrm{Al}$ foil & Beaker is covered with $\mathrm{Al}$ foil & & Beaker is covered with $\mathrm{Al}$ foil \\
\hline $\begin{array}{l}\text { After } 2 \mathrm{hrs} \mathbf{5 0} \mathbf{~ m l} \text { of deionized } \\
\text { water is added to beaker }\end{array}$ & $\begin{array}{l}\text { After } 2 \text { hrs } \mathbf{5 0} \mathbf{~ m l} \text { of deionized } \\
\text { water is added to beaker }\end{array}$ & $\begin{array}{l}\text { After } 2 \text { hrs } \mathbf{2 0 0} \mathbf{~ m l} \text { of } \\
\text { deionized water is added to } \\
\text { beaker }\end{array}$ & $\begin{array}{l}\text { After } 2 \text { hrs } \mathbf{5 0} \mathbf{~ m l} \text { of deionized } \\
\text { water is added to beaker }\end{array}$ \\
\hline $\begin{array}{l}\text { After } 4 \text { hrs hotplate is turned off } \\
\text { and system is cooled naturally } \\
\text { to room temperature }\end{array}$ & $\begin{array}{l}\text { After } 4 \text { hrs hotplate and mixer } \\
\text { are turned off and system is } \\
\text { cooled naturally to room } \\
\text { temperature }\end{array}$ & $\begin{array}{l}\text { After } 4 \text { hrs hotplate and mixer } \\
\text { are turned off and system is } \\
\text { cooled naturally to room } \\
\text { temperature }\end{array}$ & $\begin{array}{l}\text { After } 4 \text { hrs hotplate and mixer are } \\
\text { turned off and system is cooled } \\
\text { naturally to room temperature }\end{array}$ \\
\hline
\end{tabular}




\section{APPENDIX C WAX-ENCAPSULATION OF GRUBBS' RU CATALYST INSTRUCTIONS}

1. In an $\mathrm{N}_{2}$ filled glove box, seal $10.0 \mathrm{~g}$ of Paraffin wax and $0.525 \mathrm{~g}$ of Grubbs' catalyst into a scintillation vial.

2. Place $225 \mathrm{~mL}$ of water, $0.63 \mathrm{~g}$ of 0.28 wt.-\% Ethylene Maleic Anhydride (EMA) copolymer, and 1 drop of 1-octanol in a $1000 \mathrm{~mL}$ beaker. Place the beaker in an $82{ }^{\circ} \mathrm{C}$ water bath and stir for $10 \mathrm{~min}$ at $900 \mathrm{rpm}$. Place the vial in the water bath as well.

3. After 10 min the wax should have melted. Shake the vial and dump its contents into the solution. Continue stirring for $2 \mathrm{~min}$, then dump $600 \mathrm{~mL}$ of ice water into the solution and turn off the stirrer.

4. Separate the suspension of wax encapsulated catalyst under vacuum with a coarse-fritted filter. 


\section{APPENDIX D ANSYS INPUT CODE}

! Dr. Barbero, Dr. Mayugo's and Kevin Ford MODEL USING PERIODIC BC'S -symmetric

/PREP7

/VIEW,1,1,2,3

/UNITS,USER

!*

ET,1,SOLID186

!--------User inputs-------------

! geometry of the cell

nlayer $=16$

!total \#, even for symm. laminates

tk $=1$

!mm

$\mathrm{a}=1$

!mm

$\mathrm{b}=1$

$\mathrm{c}=$ nlayer*tk $/ 2$

D3 $=0$

! 1:3D RVE; 0:LAMINATED (epsilon_z=free)

$\mathrm{SYMM}=1$

! 1:YES;

$0: \mathrm{NO}$

*IF,D3,EQ,1,THEN

$\mathrm{SYMM}=0$

! 3D MODEL CANNOT BE SYMMETRIC

*ENDIF

lstep $=10$

sstep $=3$

iter $=100$

! \# sub_steps

! \# iterations

calcheal=lstep*sstep

updateheal $=2 * 1$ step* ${ }^{*}$ sstep

!strain for first loading

epxx $=0.00 \quad$ ! horizontal strain

epyy $=0.00 \quad$ ! vertical strain

epxy $=0.01125 \quad$ ! shear strains

!strain for second loading

epxx2 $=0.00 \quad$ ! horizontal strain

epyy $2=0.00 \quad$ ! vertical strain

epxy2 $=0.01125 \quad$ ! shear strains

!unloading strain factor

unld $=0.01$

TB,STAT,1,,18, ! NUMBER OF STATE VARIABLES

TBTEMP, 0

TBDATA,,,,,,

TBDATA,,,,,,, 
TBDATA,,,,,calcheal,updateheal,

TBDE,USER, $1,$,

TB,USER, $1,1,45$, ! version 3.0, 40 variables

TBTEMP, 0

! The 45 variables for the USERMAT3d subroutine healing or below

\begin{tabular}{|c|c|c|c|c|c|c|}
\hline E11 & E22 & E33 & G23(F & 44) $\mathrm{G} 1$ & 3(E55) & G12(E \\
\hline p23 & p13 & p12 & $\mathrm{J} 11$ & $\mathrm{~J} 22$ & $\mathrm{~J} 33$ & \\
\hline B11 & B22 & B33 & A1 & A2 & A3 & \\
\hline cld & $\mathrm{c} 2 \mathrm{~d}$ & gmma & & Dc1 & Dc2 & Dc3 \\
\hline F1_b & F2_b & F3_b & F4_b & F5_b & F6_b & \\
\hline F7_b & F8_b & $\mathrm{N} / \mathrm{U}$ & $\mathrm{clp}$ & $c 2 p$ & R0 & 0.347607 \\
\hline $\begin{array}{l}\text { cnvtol } \\
\text { c3h }\end{array}$ & $\begin{array}{l}\text { ncont } \\
\text { c4h }\end{array}$ & $\begin{array}{l}\text { tita } \\
\text { c5h }\end{array}$ & iEDP & clh & $\mathrm{c} 2 \mathrm{~h}$ & \\
\hline
\end{tabular}

TBDATA,,30572,8699,8699,2858,2858,2858

TBDATA, $0.251,0.251,0.251,0.00230299,0.0410099,0.0410099$

TBDATA,,0,0.0611882,0.0611882,1.32801,1,1

TBDATA, ,0.030807,-0.95384,0.000582873,0.98,0.98,0.98

TBDATA,,422.694,421.469,63.8659,63.8659,1,152.012

TBDATA,,74.6917,74.6917,0,-0.434411,0.0347361,0.565589

TBDATA,,1e-06,0,0,0,0,3.8122,

TBDATA,,-5.3876,1.539,1,

$\mathrm{R}, 1$

ET, 1, 186, 0, 0, 0, 0, 0, 0

KEYOPT, $1,7, \quad 0$

KEYOPT, $1,8, \quad 0$

KEYOPT, 1, 9, 0

KEYOPT, 1, 10, 0

KEYOPT, 1, 11, 0

LOCAL, 11,0,0.,0.,0.,-45, ! layer \#1

LOCAL, $12,0,0,, 0 ., 0 ., 45$, ! layer \#2

LOCAL, 13,0,0.,0.,0.,0, ! layer \#3

LOCAL, 14,0,0.,0.,0.,90, ! layer \#4

LOCAL, 15,0,0.,0.,0.,0, ! layer \#5

LOCAL, 16,0,0.,0.,0.,90, ! layer \#6

LOCAL, 17,0,0.,0.,0.,0, ! layer \#7

LOCAL, 18,0,0.,0.,0.,90, ! layer \#8

csys, 0

! MUST return to the 0 coordinate system 


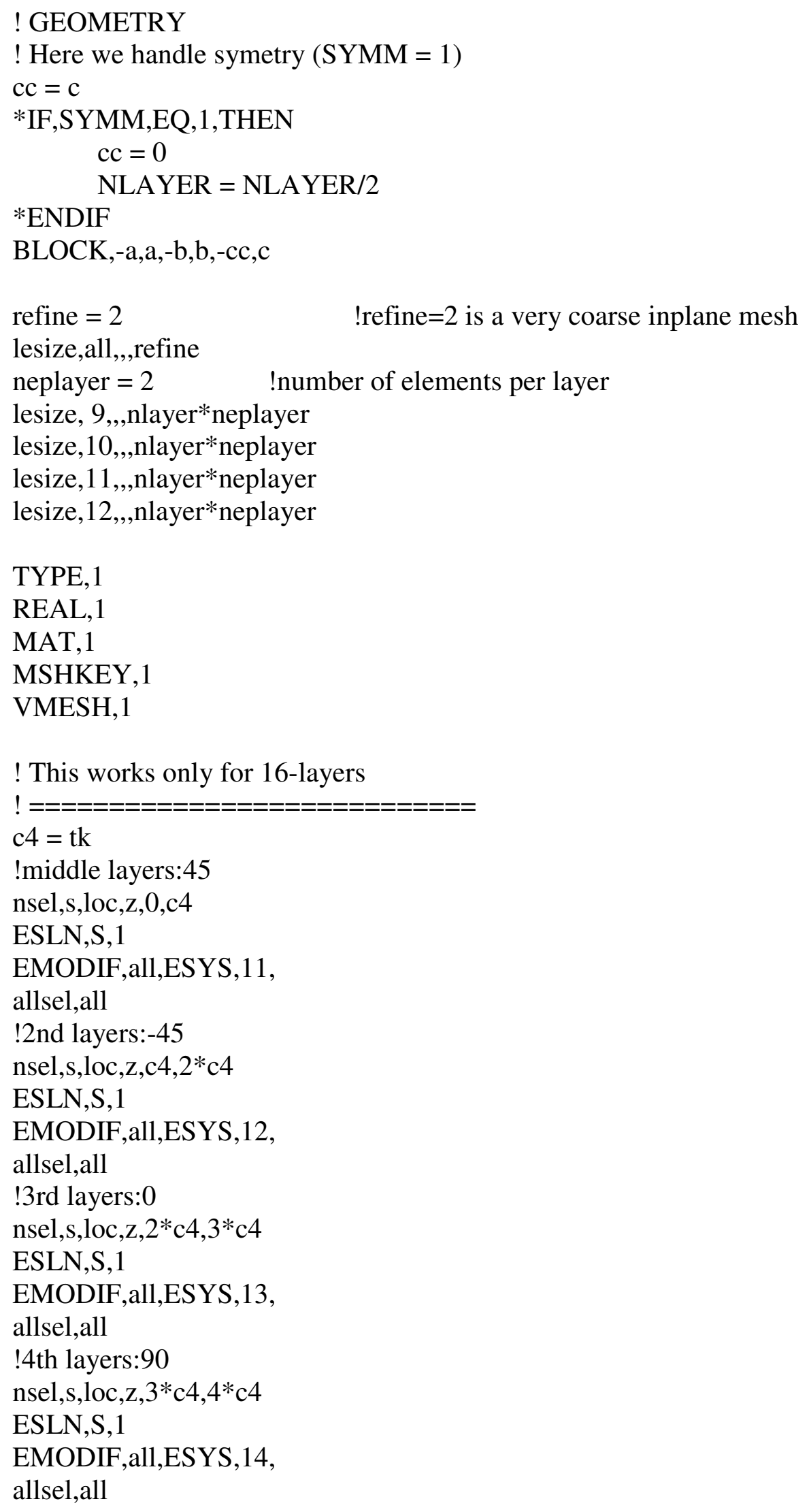


!5th layers:0

nsel,s,loc,z,4*c4,5*c4

ESLN,S,1

EMODIF,all,ESYS, 15,

allsel,all

!6th layers:90

nsel,s,loc,z,5*c4,6*c4

ESLN,S, 1

EMODIF,all,ESYS, 16,

allsel,all

!7th layers:0

nsel,s,loc,z,6*c4,7*c4

ESLN,S,1

EMODIF,all,ESYS, 17,

allsel,all

!8th layers:90

nsel,s,loc,z,7*c4,8*c4

ESLN,S, 1

EMODIF,all,ESYS, 18, allsel,all

*IF,SYMM,EQ,0,THEN

nsel,s,loc,z,-c4,0

ESLN,S, 1

EMODIF,all,ESYS, 11, allsel,all

nsel,s,loc,z,-2*c4,c4

ESLN,S,1

EMODIF,all,ESYS, 12,

allsel,all

nsel,s,loc,z,-3*c4,-2*c4

ESLN,S,1

EMODIF,all,ESYS, 13,

allsel,all

nsel,s,loc,z,-4*c4,-3*c4

ESLN,S,1

EMODIF,all,ESYS, 14, allsel,all

nsel,s,loc,z, $-5 * \mathrm{c} 4,-4 * \mathrm{c} 4$

ESLN,S,1

EMODIF,all,ESYS, 15, allsel,all

nsel,s,loc,z,-6*c4,-5*c4

ESLN,S,1 


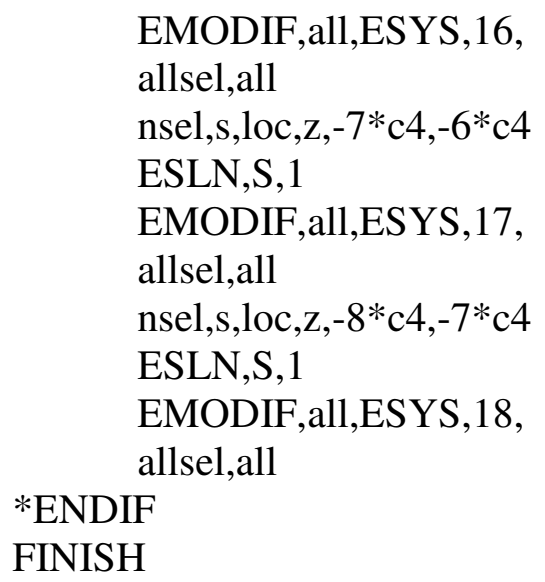

! Boundary conditions

NSEL,S,LOC,Z,0

$\mathrm{D}$, all,UZ

NSEL,R,LOC,Y,0

NSEL,R,LOC,X,0 
D,all,all ! middle node

ALLSEL,ALL

! CREATE COMPONENT SET: periodic edge and periodic sides

! select edges $a b$

nsel,s,loc, $\mathrm{x},-\mathrm{a}$

nsel,r,loc,y,-b

cm,anbn_nodes,node

nsel,s,loc, $x, a$

nsel,r,loc,y,-b

cm,apbn_nodes,node

nsel, s,loc, $x,-a$

nsel,r,loc,y,b

cm,anbp_nodes,node

nsel, s,loc, $\mathrm{x}, \mathrm{a}$

nsel,r,loc,y,b

cm,apbp_nodes,node

! select side a and -a -----------------

nsel,s,loc, $x,-a$

cmsel,u,anbn_nodes

cmsel,u,anbp_nodes

cm,an_nodes,node

nsel,s,loc, $\mathrm{x}, \mathrm{a}$

cmsel,u,apbn_nodes

cmsel,u,apbp_nodes

cm,ap_nodes,node

! select side $\mathrm{b}$ and $-\mathrm{b}$

nsel,s,loc, y,-b

cmsel,u,anbn_nodes

cmsel,u,apbn_nodes

cm,bn_nodes,node

nsel,s,loc,y,b

cmsel,u,anbp_nodes

cmsel,u,apbp_nodes

cm,bp_nodes,node

cmsel,all

ALLSEL,ALL 


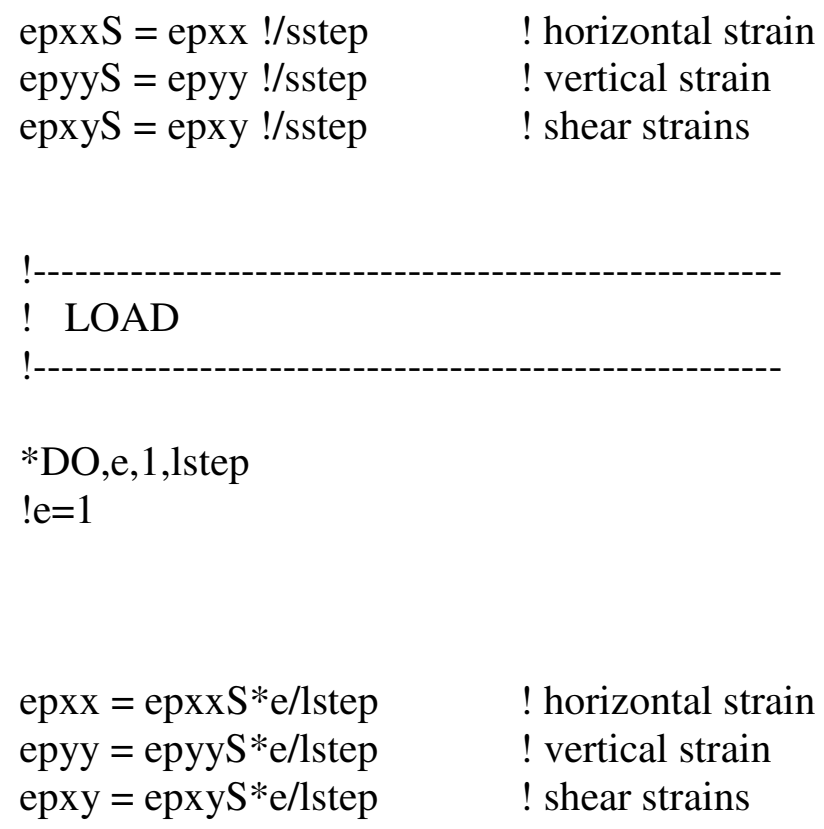

! Periodic conditions of edges (2 couple of edges)

pos_node $=$

neg_node $=$

cmsel,s,anbn_nodes

*get,num_nodes,node,0,count,max

*do,i,1,num_nodes, 1

cmsel,s,anbn_nodes

! neg_node is undefined; use lowest active node number when $\mathrm{i}=1$

*if,i,ne, 1 , then

*else neg_node=ndnext $($ neg_node $)$

*get,neg_node,node,0,num,min

*endif

! get $\mathrm{x}, \mathrm{y}, \mathrm{z}$ locations of current node in active coord system

$\mathrm{x}_{-}=\mathrm{nx}($ neg_node $)$

$y_{-}=$ny $($neg_node $)$

$\mathrm{z}_{-}=\mathrm{nz}($ neg_node $)$

cmsel,s,apbp_nodes

! get closest node from component neg_a2

pos_node $=\operatorname{node}\left(-x_{-},-y_{-}, z_{-}\right)$

ceeq $=$ ceeq +1

ce,ceeq, $($ epxx $*(a * 2))+($ epxy*(b*2)),neg_node,ux,-1,pos_node,ux, 1

ceeq $=$ ceeq +1

ce,ceeq,(epyy*(b*2))+(epxy*(a*2)),neg_node,uy,-1,pos_node,uy, 1 
*enddo

pos_node $=$

neg_node $=$

cmsel,s,anbp_nodes

*get,num_nodes,node,0,count,max

*do,i,1,num_nodes, 1

cmsel,s,anbp_nodes

! neg_node is undefined; use lowest active node number when $\mathrm{i}=1$

*if,i,ne, 1 , then

*else neg_node=ndnext(neg_node)

*get,neg_node,node,0,num,min

*endif

! get $\mathrm{x}, \mathrm{y}, \mathrm{z}$ locations of current node in active coord system

$x_{-}=n x($ neg_node $)$

$\mathrm{y}_{-}=$ny (neg_node)

$\mathrm{z} \_=$nz(neg_node)

cmsel,s,apbn_nodes

! get closest node from component neg_a2

pos_node $=\operatorname{node}\left(-x_{-},-y_{-}, z_{-}\right)$

ceeq $=$ ceeq +1

ce,ceeq, $($ epxx*(a*2))+(-epxy*(b*2)),neg_node,ux,-1,pos_node,ux,1

ceeq $=$ ceeq +1

*enddo

ce,ceeq,(epyy*(b*2))+(-epxy*(a*2)),pos_node,uy,-1,neg_node,uy, 1

! Periodic contitions of sides (9 couple of sides)

pos_node $=$

neg_node $=$

cmsel,s,ap_nodes

*get,num_nodes,node, 0, count,max

*do,i,1,num_nodes, 1

cmsel,s,an_nodes

! neg_node is undefined; use lowest active node number when $\mathrm{i}=1$

$*$ if,i,ne, 1 , then

$$
\text { neg_node=ndnext(neg_node) }
$$

*else

*get,neg_node,node,0,num,min

*endif

! get $\mathrm{x}, \mathrm{y}, \mathrm{z}$ locations of current node in active coord system

$\mathrm{x} \_$_nx (neg_node)

$\mathrm{y}_{-}=$ny $($neg_node $)$

$z_{-}=n z($ neg_node $)$ 


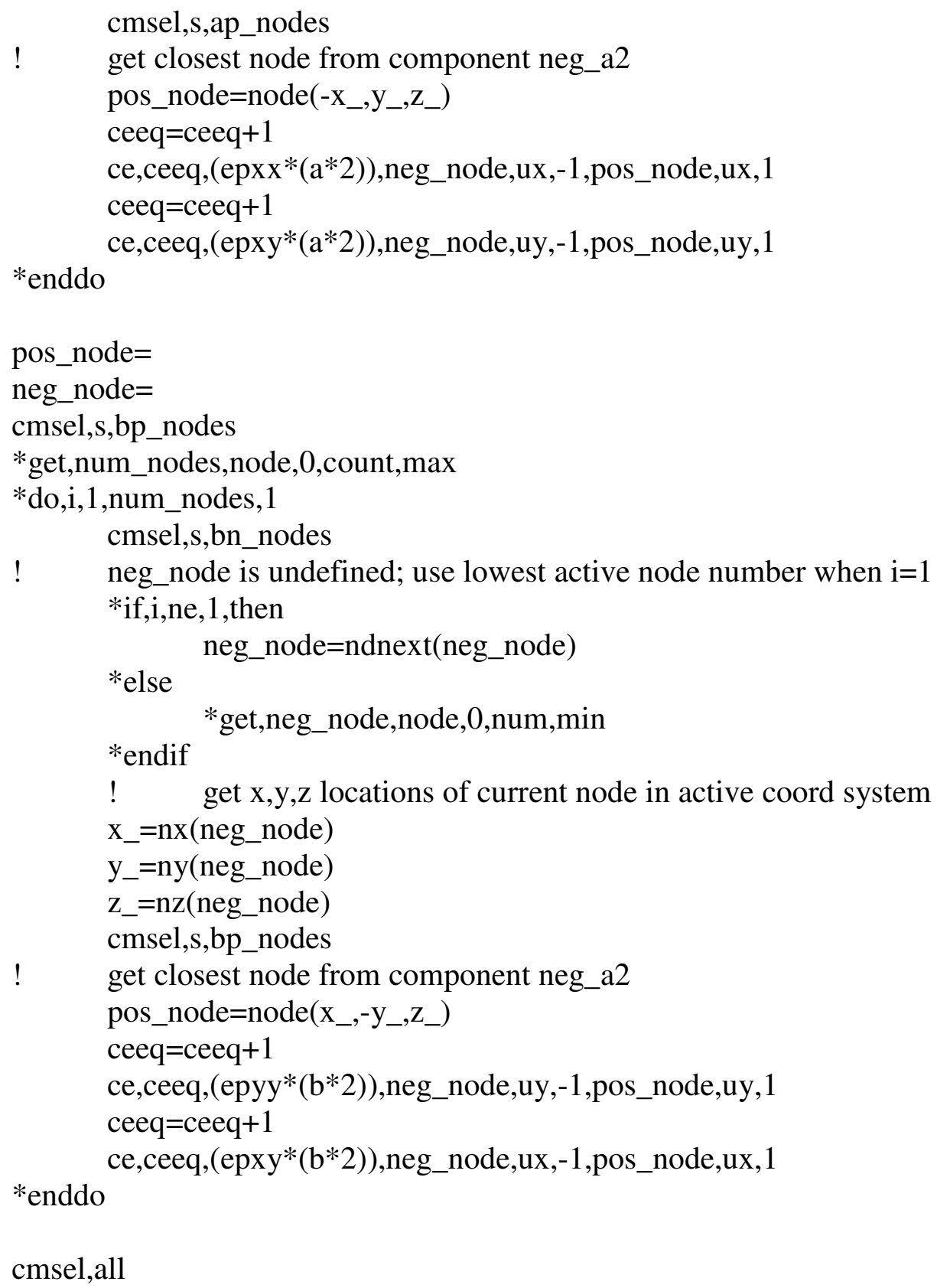


epxxSu $=$ epxxS*unld ! horizontal strain

epyySu $=$ epyy $S^{*}$ unld $!$ vertical strain

epxySu $=$ epxyS*unld $!$ shear strains

*DO,e, 1 ,lstep

!e $=1$

epxx $=$ epxxS-((epxxS-epxxSu)*e/lstep $)$

! horizontal strain

epyy $=$ epyyS-((epyyS-epyySu)*e/lstep)

! vertical strain

epxy $=$ epxyS-((epxyS-epxySu)*e/lstep)

! shear strains

CEDELE,all

ceeq $=0$

! Periodic conditions of edges ( 2 couple of edges)

pos_node $=$

neg_node $=$

cmsel,s,anbn_nodes

*get,num_nodes,node, 0 ,count,max

*do,i,1,num_nodes, 1

cmsel,s,anbn_nodes

! neg_node is undefined; use lowest active node number when $\mathrm{i}=1$

*if,i,ne, 1 , then

neg_node=ndnext(neg_node)

*else

*get,neg_node,node, 0, num,min

*endif

! get $\mathrm{x}, \mathrm{y}, \mathrm{z}$ locations of current node in active coord system

$\mathrm{x}_{-}=\mathrm{nx}($ neg_node $)$

$\mathrm{y}_{-}=$ny $($neg_node $)$

$\mathrm{z}_{-}=\mathrm{nz}$ (neg_node)

cmsel,s,apbp_nodes

! get closest node from component neg_a2

pos_node $=\operatorname{node}\left(-\mathrm{x}_{-},-\mathrm{y}_{-}, \mathrm{z}_{-}\right)$

ceeq $=$ ceeq +1

ce,ceeq, $($ epxx*(a*2))+(epxy*(b*2)),neg_node,ux,-1,pos_node,ux,1

ceeq $=$ ceeq +1

ce,ceeq, $($ epyy*(b*2))+(epxy*(a*2)),neg_node,uy,-1,pos_node,uy,1

*enddo

pos_node $=$

neg_node $=$

cmsel,s,anbp_nodes 
*get,num_nodes,node,0,count,max

*do,i,1,num_nodes, 1

cmsel,s,anbp_nodes

! neg_node is undefined; use lowest active node number when $\mathrm{i}=1$

*if,i,ne, 1 , then

*else neg_node=ndnext(neg_node)

*get,neg_node,node,0,num,min

*endif

! get $\mathrm{x}, \mathrm{y}, \mathrm{z}$ locations of current node in active coord system

$\mathrm{x}_{-}=\mathrm{nx}($ neg_node $)$

$\mathrm{y}_{-}=$ny $($neg_node $)$

$\mathrm{z}_{-}=\mathrm{nz}($ neg_node $)$

cmsel,s,apbn_nodes

! get closest node from component neg_a2

pos_node $=\operatorname{node}\left(-\mathrm{x}_{-},-\mathrm{y}_{-}, \mathrm{z}_{-}\right)$

ceeq $=$ ceeq +1

ce,ceeq, $\left(\operatorname{epxx} *\left(a^{*} 2\right)\right)+(-e p x y *(b * 2))$, neg_node,ux,-1,pos_node,ux, 1

ceeq $=$ ceeq +1

ce,ceeq, $($ epyy*(b*2))+(-epxy*(a*2)),pos_node,uy,-1,neg_node,uy, 1

*enddo

! Periodic contitions of sides (9 couple of sides)

pos_node $=$

neg_node $=$

cmsel,s,ap_nodes

*get,num_nodes,node,0,count,max

*do,i,1,num_nodes, 1

cmsel,s,an_nodes

! neg_node is undefined; use lowest active node number when $\mathrm{i}=1$

*if,i,ne, 1 , then

neg_node=ndnext(neg_node)

*else

*get,neg_node,node,0,num,min

*endif

! get $\mathrm{x}, \mathrm{y}, \mathrm{z}$ locations of current node in active coord system

$x_{-}=n x($ neg_node $)$

$\mathrm{y}_{-}=$ny (neg_node)

$z_{-}=n z($ neg_node)

cmsel,s,ap_nodes

! get closest node from component neg_a2

pos_node $=\operatorname{node}\left(-\mathrm{x}_{-}, \mathrm{y}_{-}, \mathrm{z} \_\right)$

ceeq $=$ ceeq +1

ce,ceeq,(epxx*(a*2)),neg_node,ux,-1,pos_node,ux, 1 


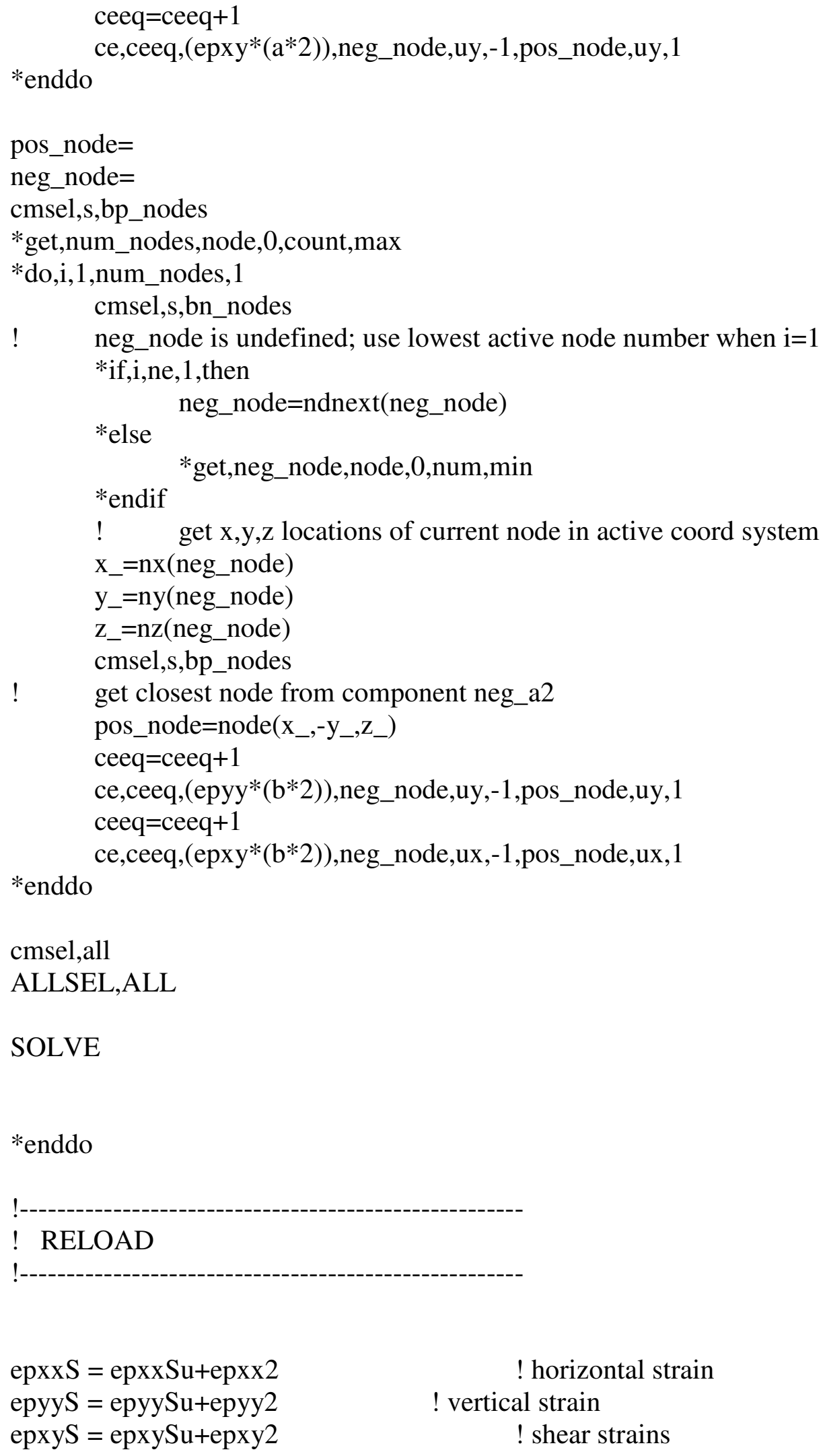

! horizontal strain

! vertical strain

! shear strains 
*DO,e,1,1step

!e $=1$

epxx = epxxS*e/lstep ! horizontal strain

epyy $=$ epyy $S *$ e/lstep ! vertical strain

epxy $=$ epxyS ${ }^{*}$ e/lstep $\quad$ ! shear strains

CEDELE,all

ceeq $=0$

! Periodic conditions of edges (2 couple of edges)

pos_node $=$

neg_node $=$

cmsel,s,anbn_nodes

*get,num_nodes,node, 0, count,max

*do,i,1,num_nodes, 1

cmsel,s,anbn_nodes

! neg_node is undefined; use lowest active node number when $\mathrm{i}=1$

*if,i,ne, 1 , then

*else

neg_node=ndnext(neg_node)

*get,neg_node,node, 0, num,min

*endif

! get $\mathrm{x}, \mathrm{y}, \mathrm{z}$ locations of current node in active coord system

$\mathrm{x} \_=\mathrm{nx}(\text { neg_node })$

$\mathrm{y}_{-}=$ny $($neg_node $)$

$z_{-}=n z($ neg_node)

cmsel,s,apbp_nodes

! get closest node from component neg_a2

pos_node $=\operatorname{node}\left(-x_{-},-y_{-}, z_{-}\right)$

ceeq $=$ ceeq +1

ce,ceeq, (epxx*(a*2))+(epxy*(b*2)),neg_node,ux,-1,pos_node,ux, 1

ceeq $=$ ceeq +1

ce,ceeq,(epyy*(b*2))+(epxy*(a*2)),neg_node,uy,-1,pos_node,uy,1

*enddo

pos_node $=$

neg_node $=$

cmsel,s,anbp_nodes

*get,num_nodes,node, 0 ,count,max

*do,i,1,num_nodes, 1

cmsel,s,anbp_nodes

! neg_node is undefined; use lowest active node number when $\mathrm{i}=1$

$*$ if,i,ne, 1 , then 


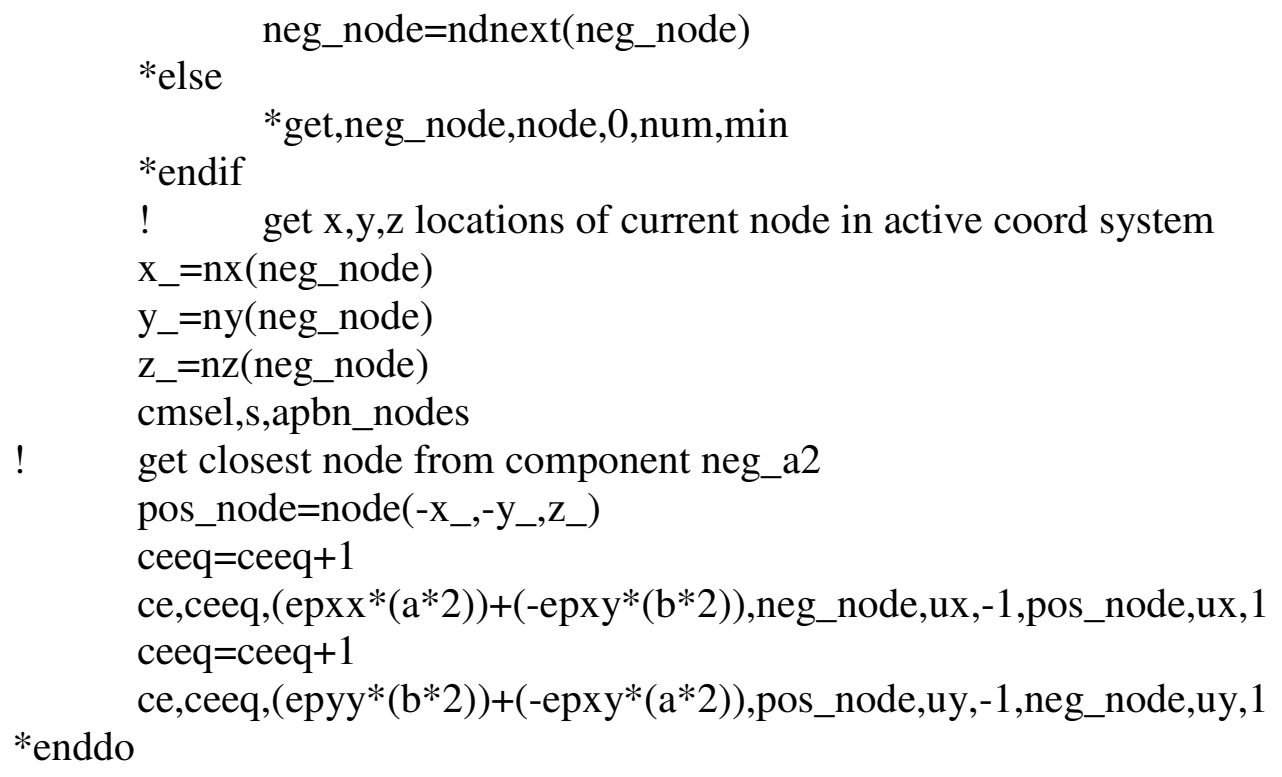

! Periodic contitions of sides (9 couple of sides)

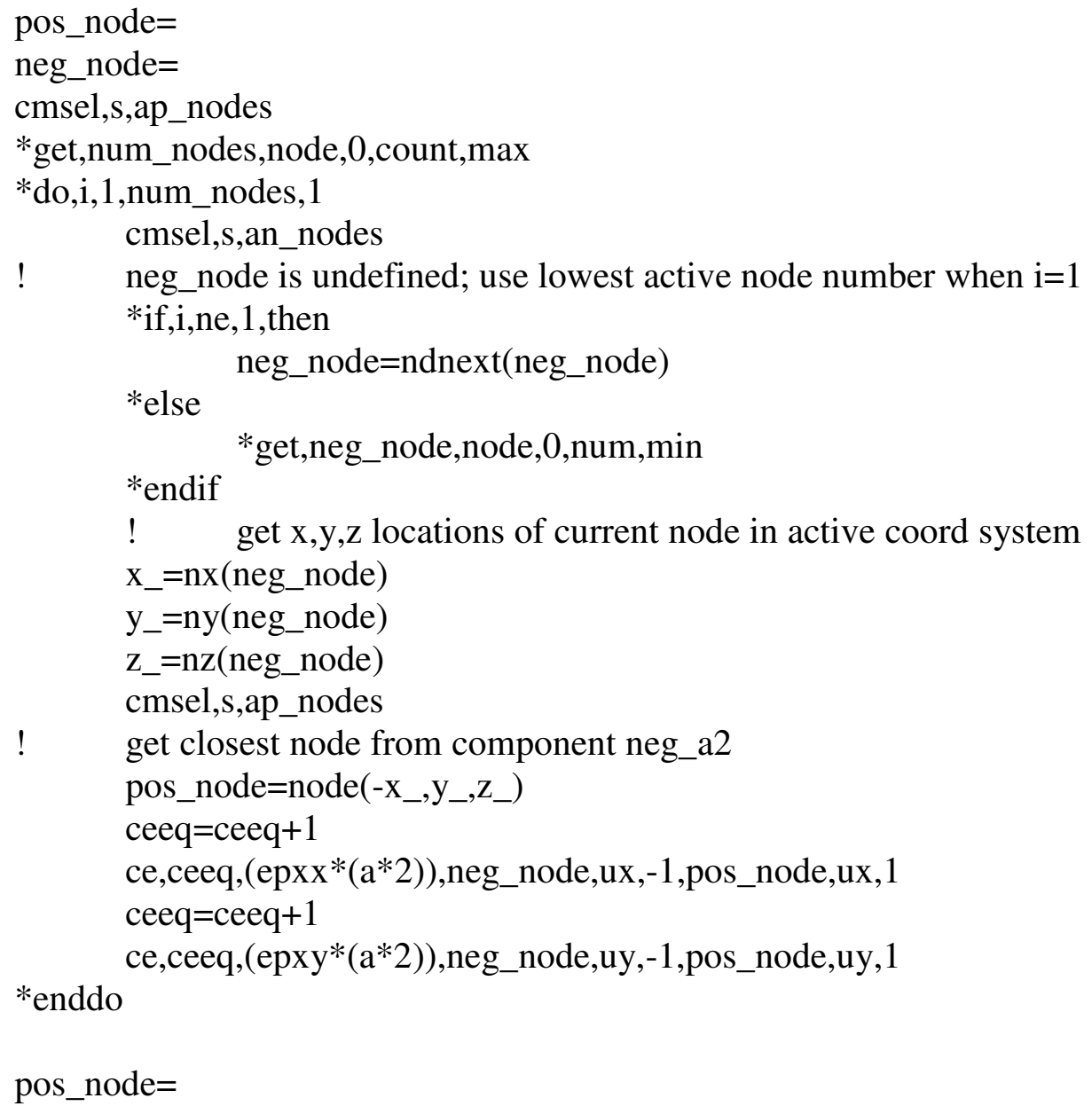




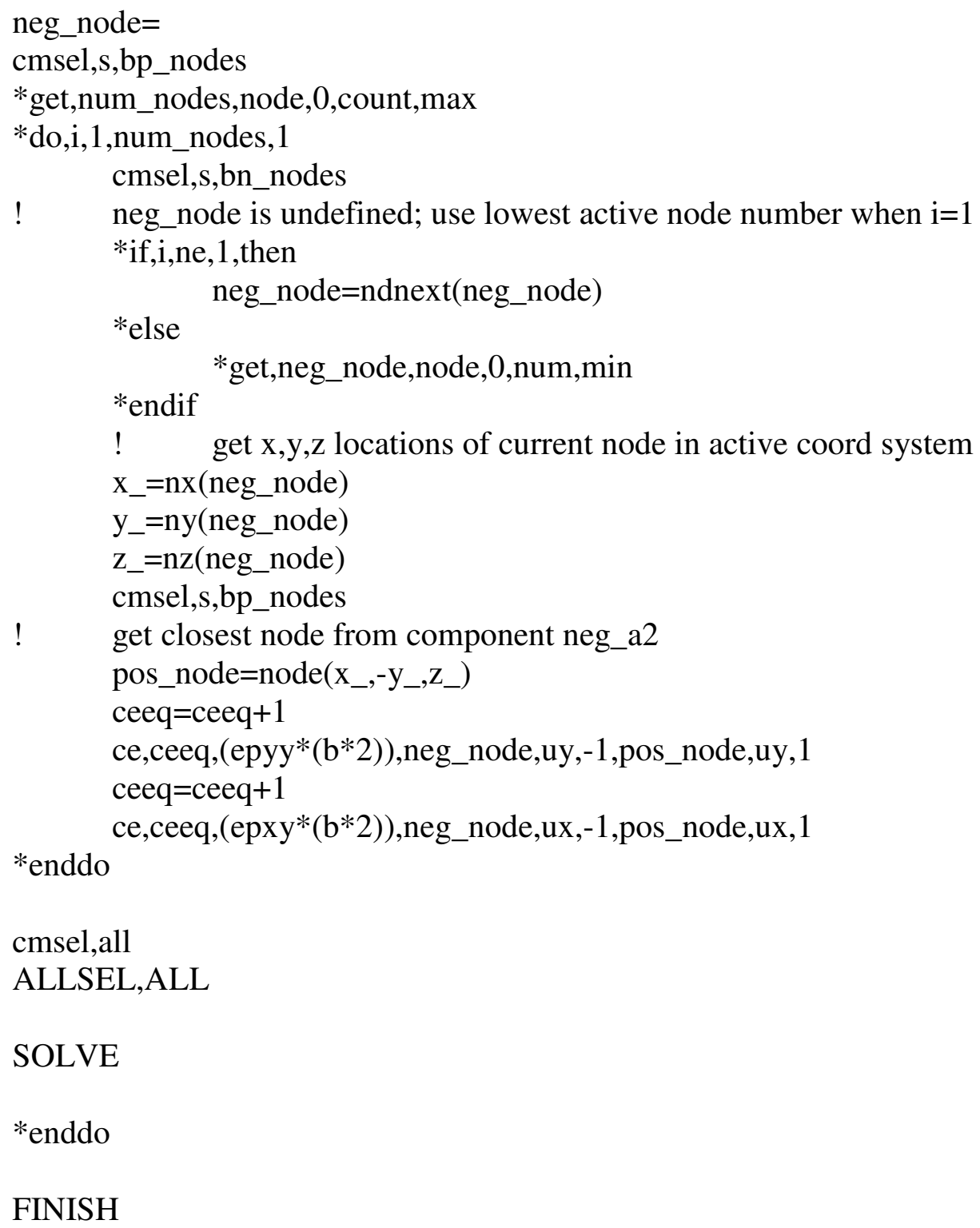

